\title{
WORKPLACE EMAIL COMMUNICATION IN NEW ZEALAND AND MALAYSIA: THREE CASE STUDIES
}

by

Jackie YEOH Lay Kean

A thesis submitted to the Victoria University of

Wellington in fulfilment of the requirements for the degree of Doctor of Philosophy

Victoria University of Wellington

2014 



\section{Abstract}

This thesis is an analysis of authentic communication between professionals at work in three workplaces (Company NZ1, NZ2 and M). The research involves a contrastive study of internal emails in two different countries (New Zealand and Malaysia) with very distinct cultures. In addition to the email corpus comprising 1745 emails, the analysis is supported by data collected using a mixed-methods approach: fieldwork observations, a questionnaire and interviews.

The analysis suggests that workplace culture influences people's linguistic and non-linguistic behaviours. A Community of Practice (CofP) approach provided insights into characteristics of workplace culture such as the participants' behaviours, language, values and beliefs. This approach also facilitated analysis of how these behaviours were negotiated among the staff members, and how practices were established to signify membership of communities of practice.

Using an adapted version of Speech Act Theory, the email messages were coded initially for their main communicative function. The next layer of analysis involved exploring the interpersonal and power dimensions of the communicative function of making requests. To this end, Spencer-Oatey's Rapport Theory was applied as the primary theoretical framework and Halliday's three metafunctions were also used to interpret the emails.

The analysis indicated that all three workplaces use email for the same communicative functions, but the proportions of usage differed. In the New Zealand workplaces, providing information is the predominant function, followed by making requests. By contrast, making requests dominates the use of email in the Malaysian workplace.

The analysis demonstrates that rapport is managed differently in the three communities of practice. The greater use of informal greetings and closings and various linguistic strategies such as modalised interrogatives and mitigating devices in one New Zealand workplace suggests that participants are attending to rapport, and that they are aware of the importance of maintaining harmonious collegial relationships when making requests of their work colleagues. On the other hand, a greater use of formal greetings and closings, bare imperatives and boosting devices in the Malaysian workplace suggests the converse. 
Furthermore, the analysis indicates that rapport is managed quite differently even when participants are from the same country (New Zealand) which practises an egalitarian culture.

The analysis also demonstrates how superiors and subordinates 'do' power and construct authority through the use of various linguistic strategies, such as imperative mood, use of the personal pronoun ' $I$ ', and boosting devices. The greater use of these linguistic strategies in the other New Zealand workplace and the Malaysian workplace suggests that more importance was placed on getting the job done than on maintaining rapport. In addition, the analysis identifies different attitudes towards the manifestation of power. In New Zealand where egalitarianism is highly valued, an overt display of power could damage work relationships. By contrast, in Malaysia, inequality in power is accepted as normal. People do not question superiors' authority, rights and entitlement to privileges. Recognising such culturally different values proved important in interpreting the data.

To conclude, this research illustrates how the use of a rapport management framework can provide new insights in relation to online workplace communication. Email not only 'does' power and performs transactional work, it also accomplishes relational work. Furthermore, the CofP approach provides insights concerning how each workplace establishes different linguistic and non-linguistic practices which form the basis of a distinct workplace culture. Finally, the analysis makes a contribution to the field of email communication from a crosscultural perspective. 


\section{Acknowledgements}

"Trust in the Lord with all your heart, and lean not on your own understanding; in all your ways acknowledge Him, and He shall direct your paths" (Prov. 3:5-6)

First, I thank the Lord for taking me through this PhD journey and 'sending' all the wonderful people who have contributed, directly and indirectly, to the successful completion of my thesis. I wish to express my gratitude to them.

To my supervisors, Professor Janet Holmes and Dr Elaine Vine, thank you for your patience, unfailing support, dedication and academic guidance. Your constant feedback on my writing, wisdom and whole-hearted involvement has supported me throughout this process.

My gratitude goes out to Dr Teoh Mei Lin and Dr KK Ng for encouraging me to do a PhD abroad. I am eternally thankful to Dr Teoh Mei Lin for your constant words of encouragement and unfailing emotional support.

A big thank you also goes out to my participants. Thank you for your time and your input. This study would not be possible without you. I am indebted to two special friends - Dr David Hutchison and Wong Sum Nuck - who vouched for my credibility. Without you, I would not have access to two data collection sites.

I must thank the School of Linguistics and Applied Language Studies for providing me with part-time employment. Special thanks go to Sarah Dunstan, Dr Patrick Coelho, Dr Angela Joe and Natalia Peterson for calling on me first when jobs become available. I also acknowledge Victoria University for the financial support provided through the Victoria PhD Submission Scholarship.

To my fellow PhD buddies - Friederike Tegge, Dr Sharon Marsden, Dr Elizaveta Tarasova, Dr Anita Jimmie, Jeremy Koay - thank you for your endless emotional support, and advice in times of need. Thank you, Dr Kieran File, for sacrificing your time to help me understand Halliday's work and for sharing a useful interview technique. A special and big thank you goes out to Susan Smith for your unfailing emotional support, comfort, encouragement and assistance through the sweet and bitter experiences throughout this memorable PhD journey. Thank you for being there when the 'going gets tough'. Thank you also to Associate 
Professor Dr Stefanie Pillai and Anna Adams for your valuable suggestions to improve my style of writing.

I would also like to acknowledge and thank my examiners - Dr Derek Wallace, Dr Judy Hunter and Professor Hans Ladegaard - for their constructive feedback and insightful comments on my thesis.

To my Kiwi family, Peter and Audrey Vernon, who not only take care of me and treat me as part of their family, but also for having me at their bach for a great summer holiday each year throughout my PhD journey.

Lastly, I want to thank my family members for their love and patience. To my mom, for your encouragement, although letting me go overseas was the hardest thing for you to do. To my sister, Julie, for your unfailing support, who gave me a nudge to pursue my PhD. Thanks to my adorable nephews, Javin and Javen, for the Facebook chats. To my brother, David and his family, thanks for a wonderful South Island holiday. To my aunt Liew and cousin, Florence, thanks for the Skype chat sessions.

This thesis is dedicated to my late uncle Liew, father and my beloved 'son', Lucky. 


\section{Tables of Contents}

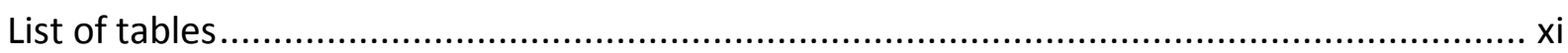

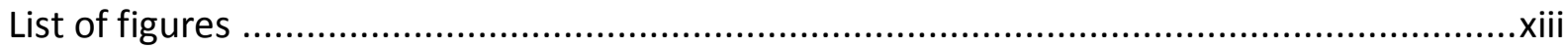

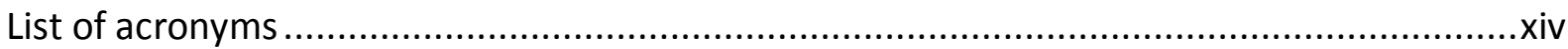

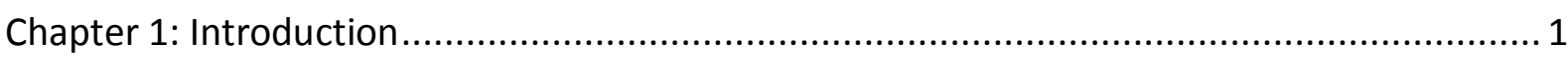

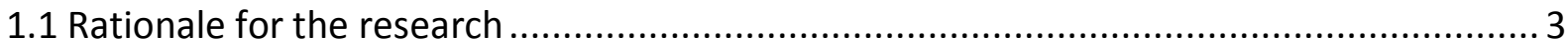

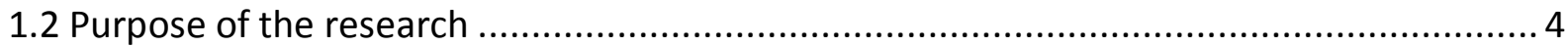

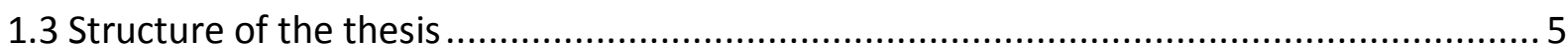

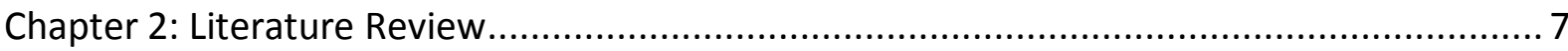

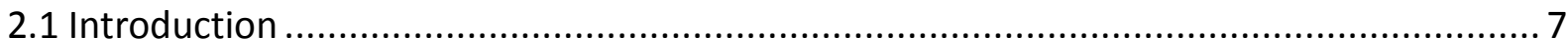

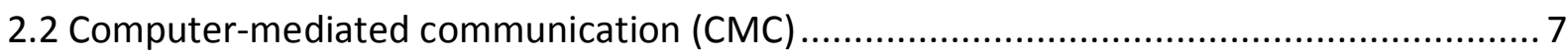

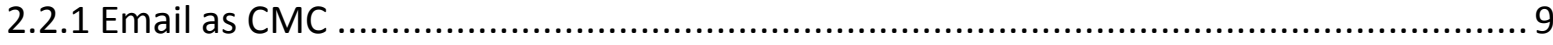

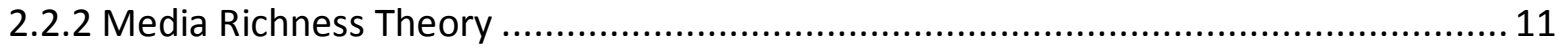

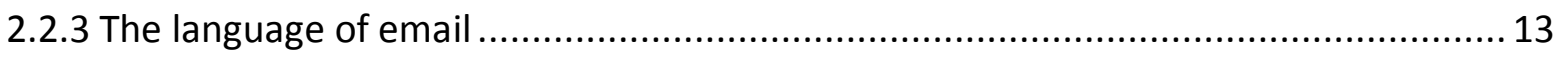

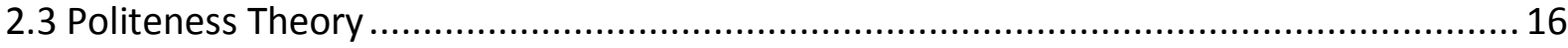

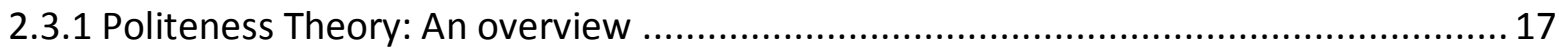

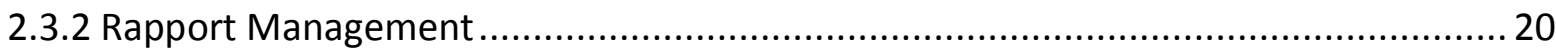

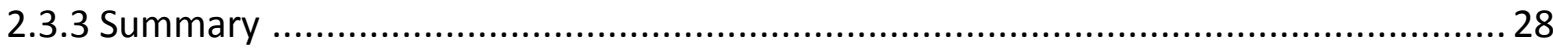

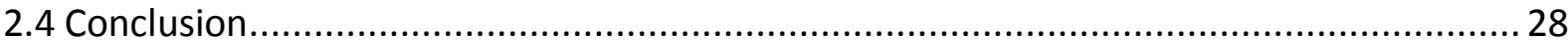

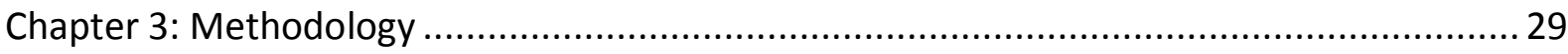

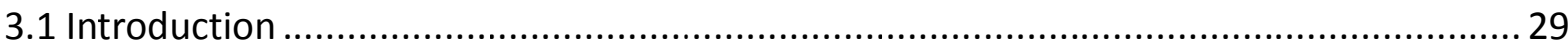

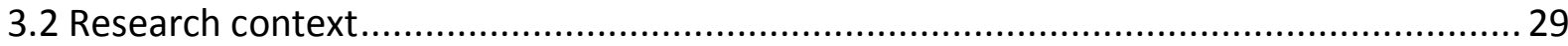

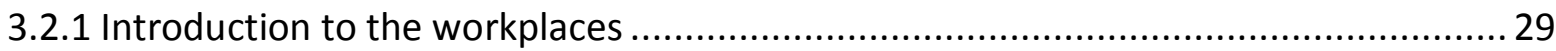

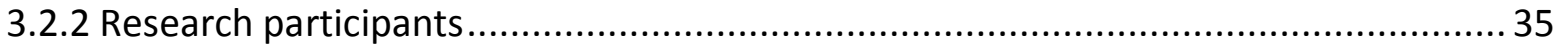

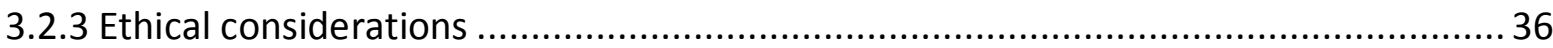

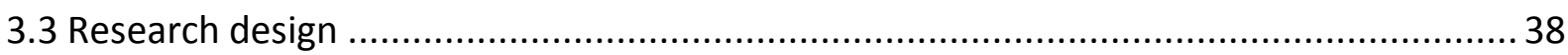

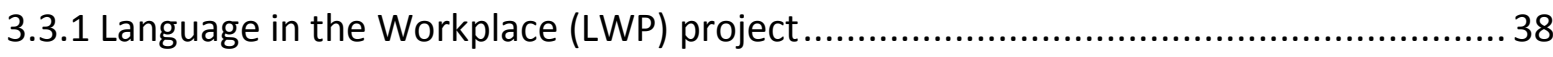

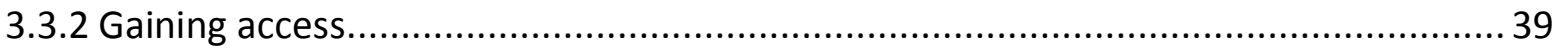

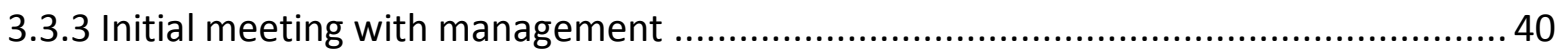

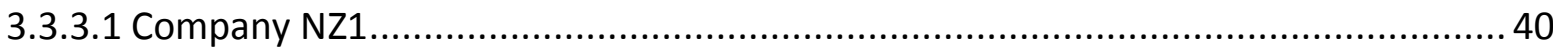

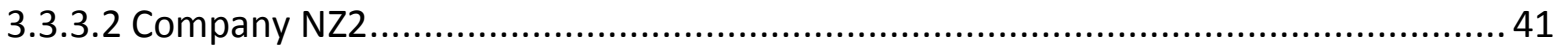

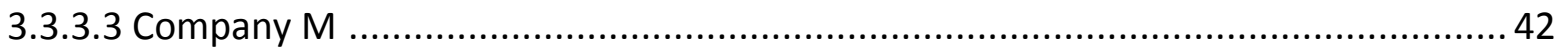




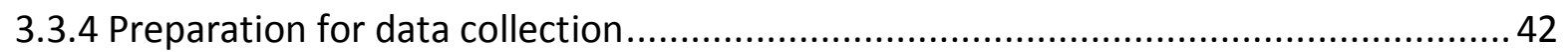

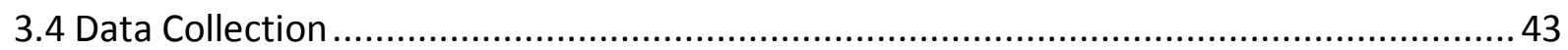

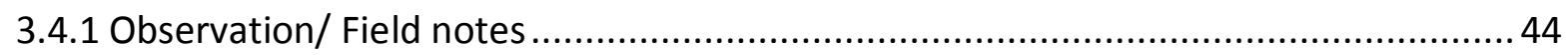

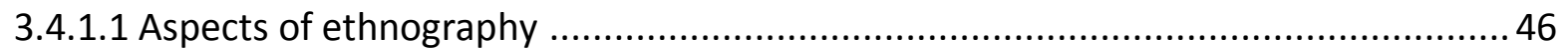

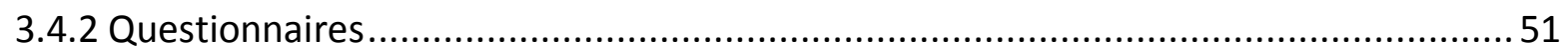

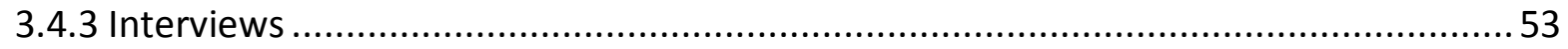

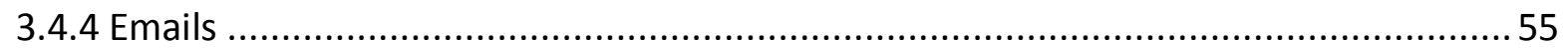

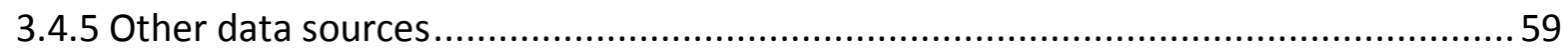

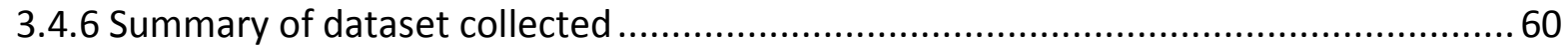

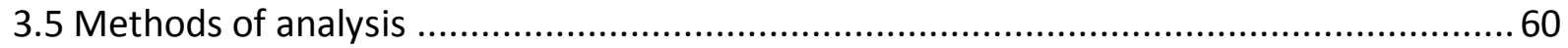

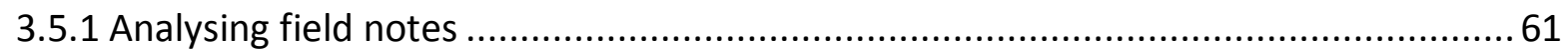

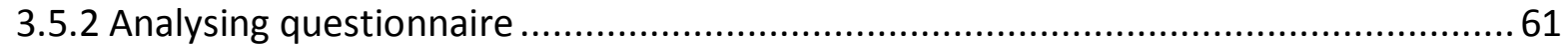

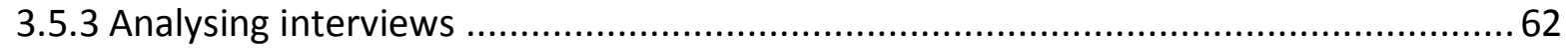

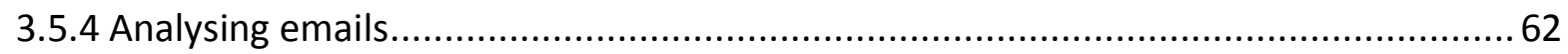

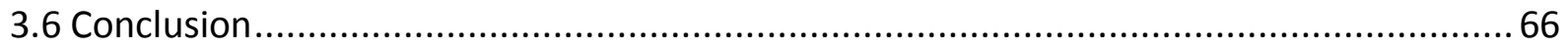

Chapter 4: The Workplaces as Communities of Practice ...................................................69

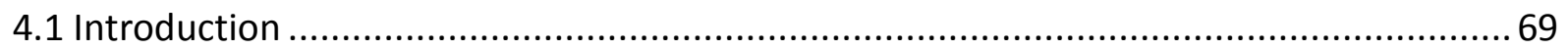

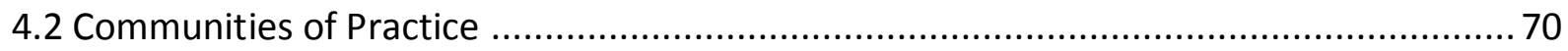

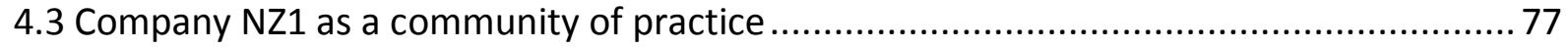

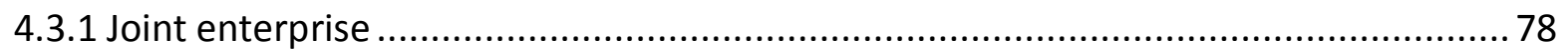

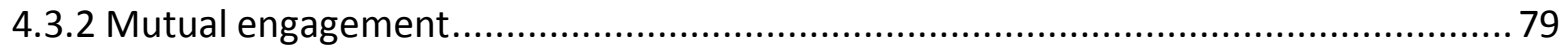

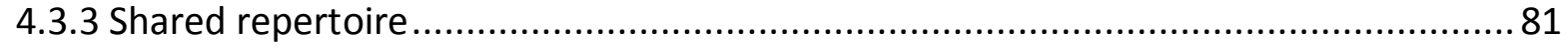

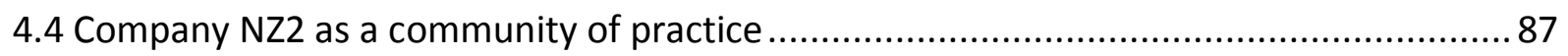

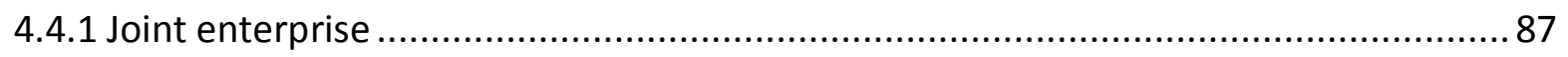

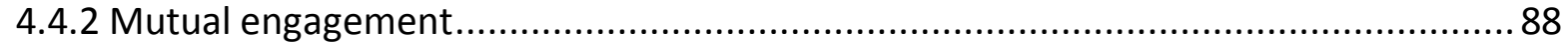

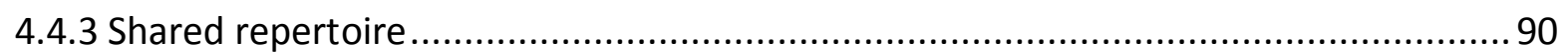

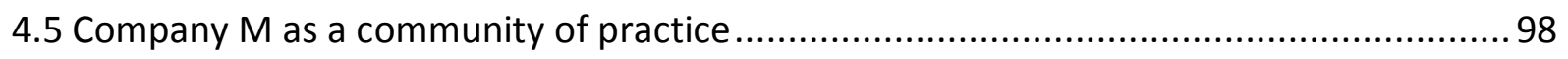

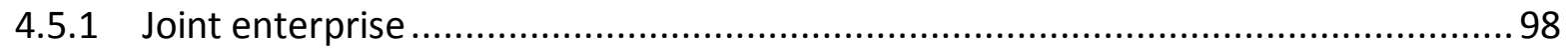

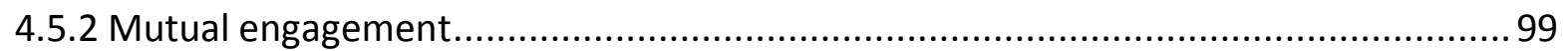

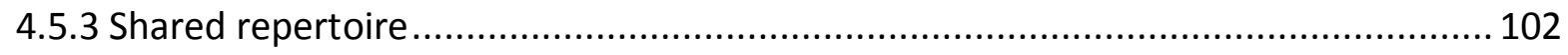

4.6 Becoming a member in a community of practice ................................................ 108

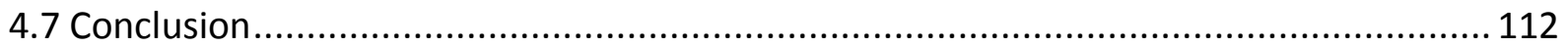


Chapter 5: Communicative Functions of Emails.

5.1 Introduction

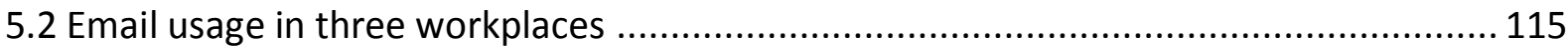

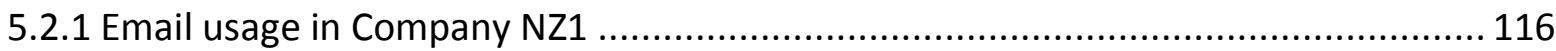

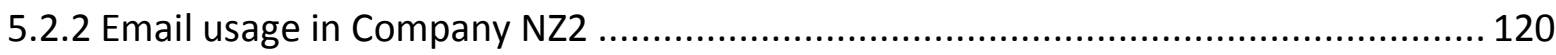

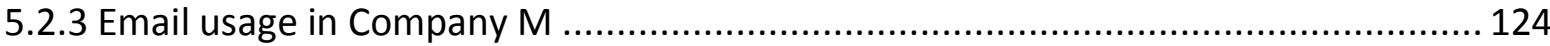

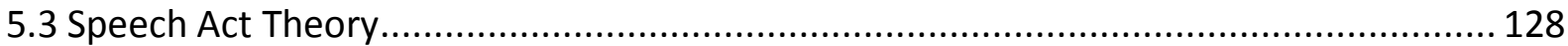

5.4 Communicative functions of emails in three workplaces .......................................... 138

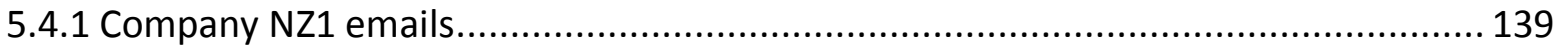

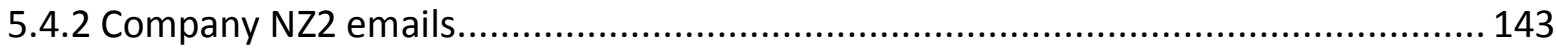

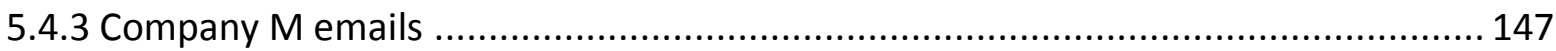

5.5 Discussion: Three workplaces compared …....................................................... 150

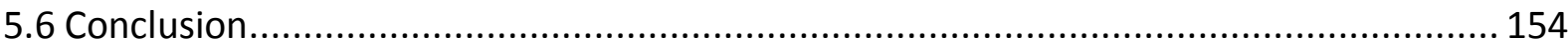

Chapter 6: How Greetings and Closings Contribute to Power Relations and Solidarity ....... 157

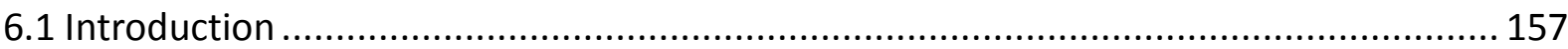

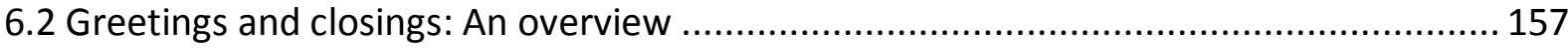

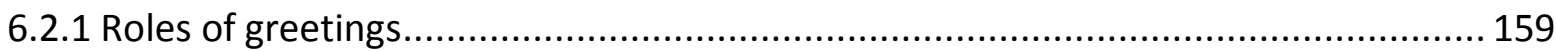

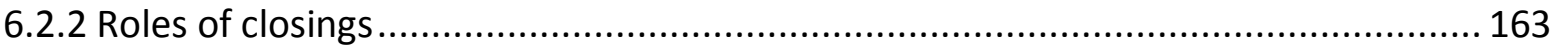

6.3 Use of greetings and closings in Company NZ1 ................................................ 165

6.4 Use of greetings and closings in Company NZ2 .................................................. 171

6.5 Use of greetings and closings in Company M..................................................... 176

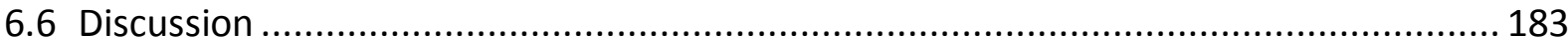

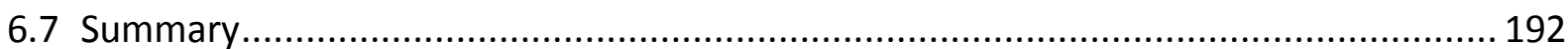

Chapter 7: Requests - constructing power relations and rapport ................................. 195

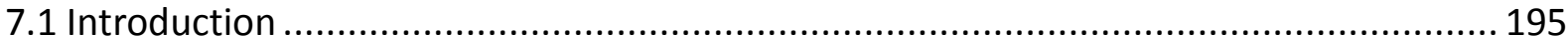

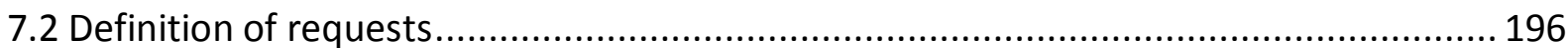

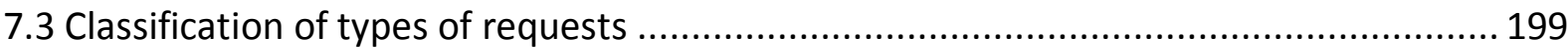

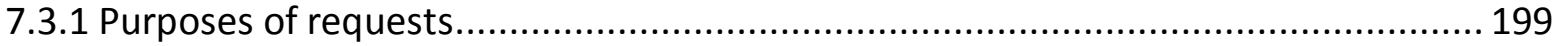

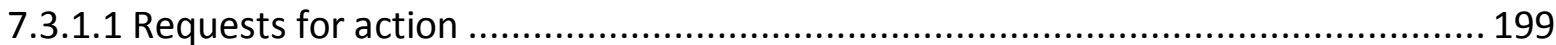

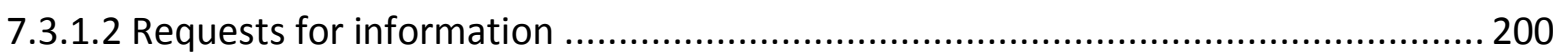

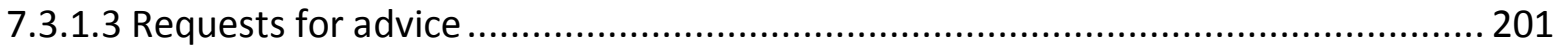

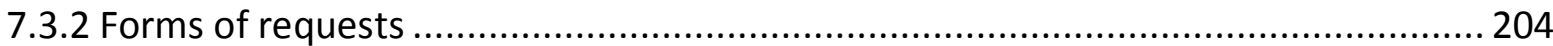




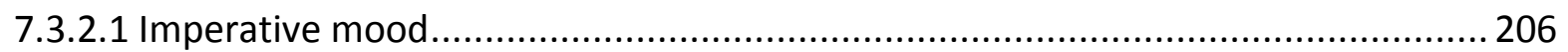

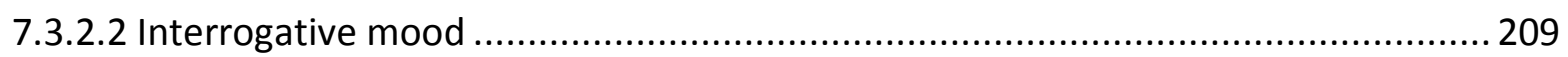

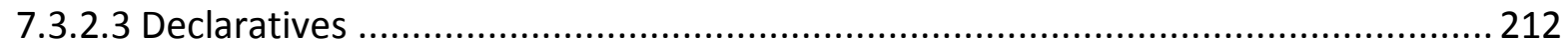

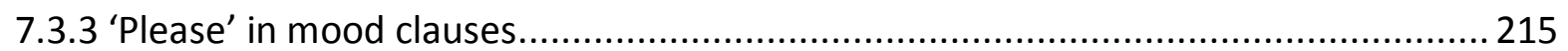

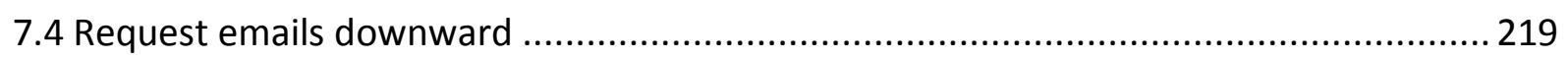

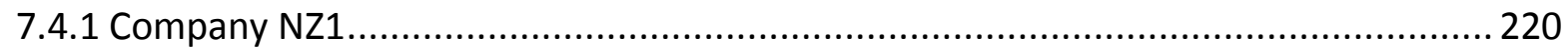

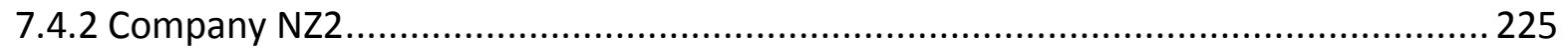

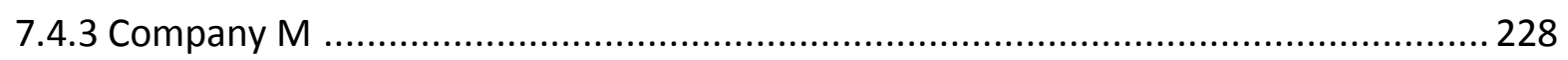

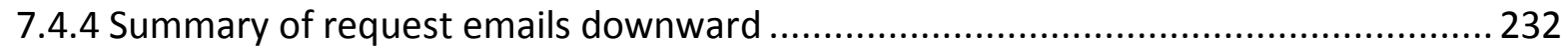

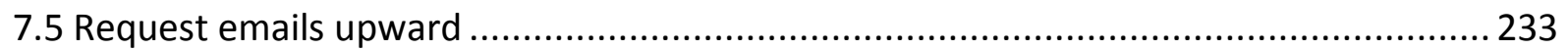

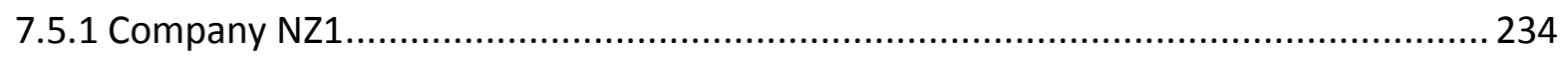

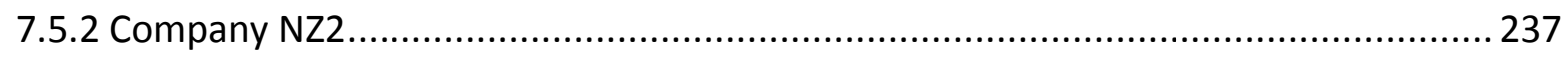

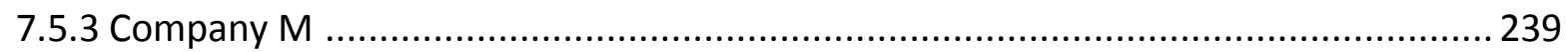

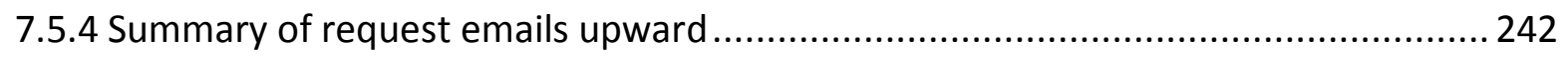

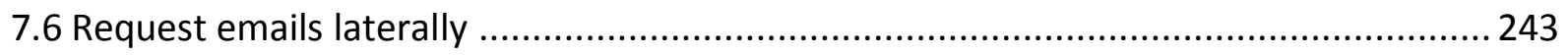

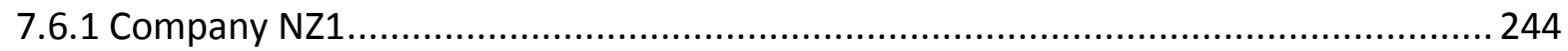

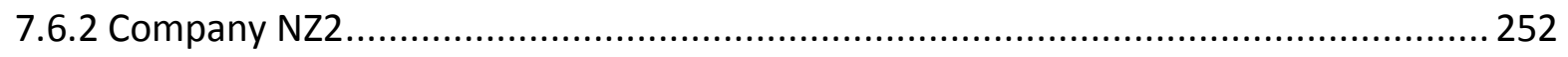

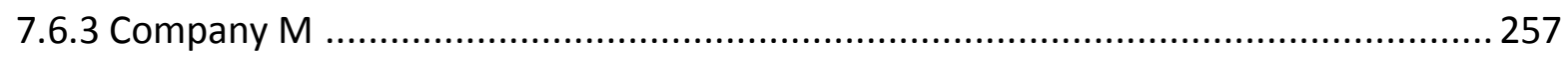

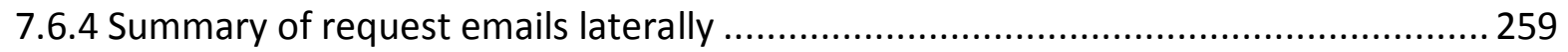

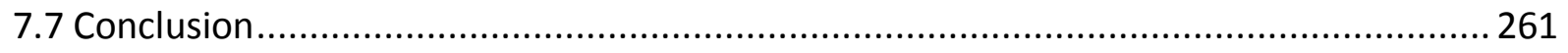

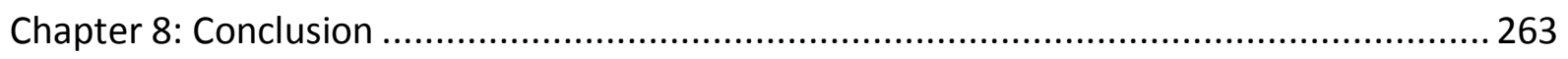

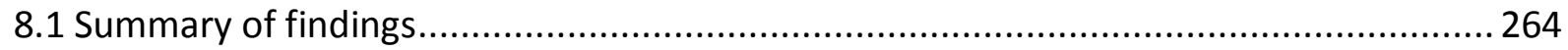

8.1.1 Research question one: Relational work in email messages ................................ 264

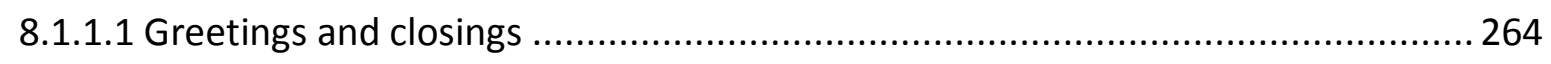

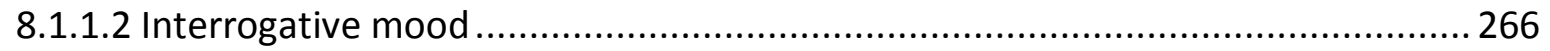

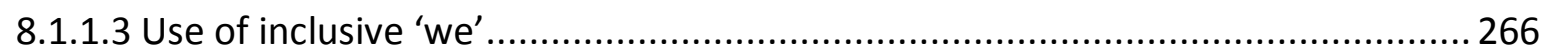

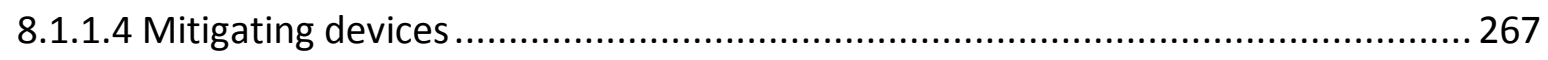

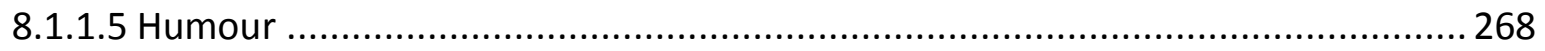

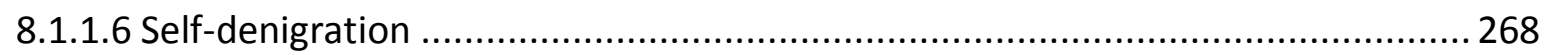

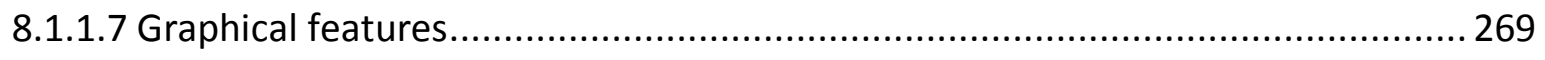

8.1.2 Research question two: Construction of power in email messages........................ 269

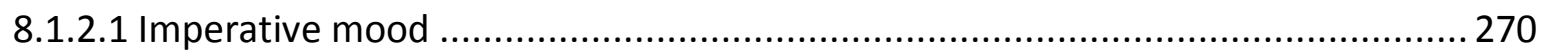


8.1.2.2 Use of personal pronoun ' $\mathrm{l}$

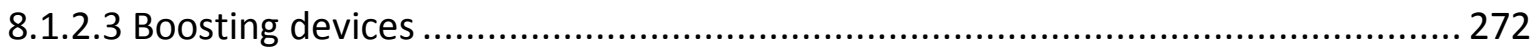

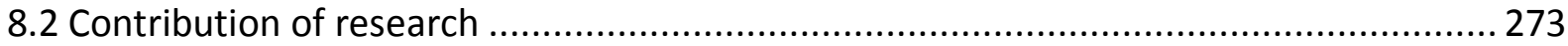

8.2.1 Contribution to research on computer-mediated communication (CMC) ............... 273

8.2.2 Contribution to research on interpersonal communication ................................... 274

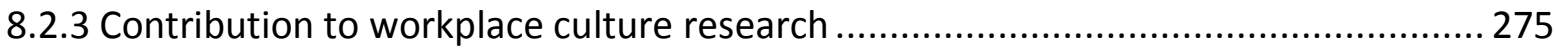

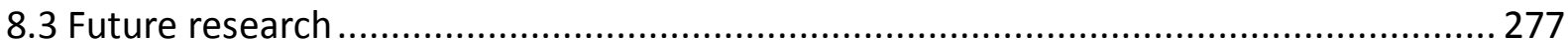

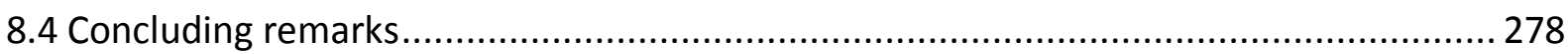

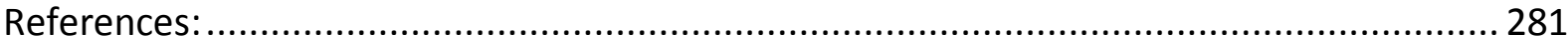

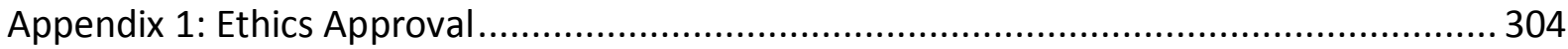

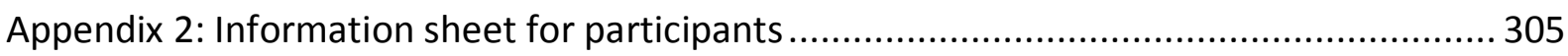

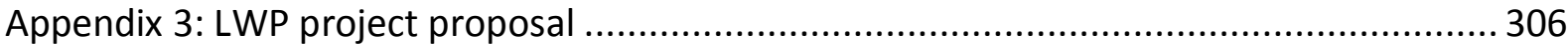

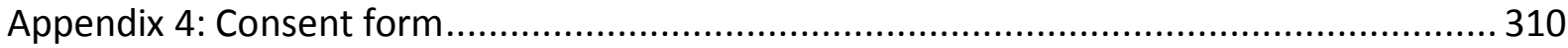

Appendix 5: Workplace email questionnaire for participants ...................................... 311

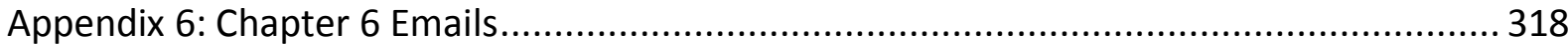

\section{List of tables}

Table 2.1 Types of synchronous and and asynchronous CMC (Maricic, 2005, p. 16) ..............8

Table 2.2 Components of Rapport Management (Spencer-Oatey, 2000c, p. 15) ................. 24

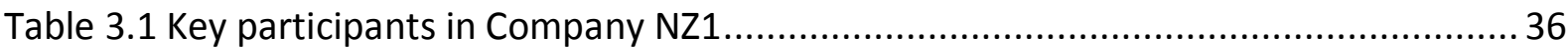

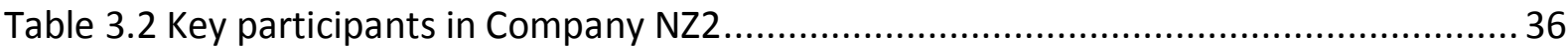

Table 3.3 Key participants in Company M........................................................................... 36

Table 3.4 Breakdown of the roles of staff members in Company NZ1, NZ2 and M..............52

Table 3.5 Composition of emails received from three workplaces ................................... 58

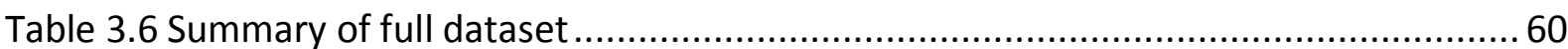

Table 3.7 Overview of how interpersonal rapport is managed through the five domains ..... 65

Table 5.1 Amount of time spent on email daily by participants in Company NZ1 ............... 116

Table 5.2 Frequency of reported use of email in Company NZ1 ................................... 117

Table 5.3 Amount of time spent on email daily by participants in Company NZ2 ............... 120

Table 5.4 Frequency of reported use of email in Company NZ2 ..................................... 122 
Table 5.5 Amount of time spent on email daily by participants in Company M. 125

Table 5.6 Frequency of reported use of email in Company M........................................ 126

Table 5.7 Emails by key participants in Company NZ1 .................................................. 139

Table 5.8 Main communicative functions of emails in Company NZ1 and Chi-square results

Table 5.9 Results for Chi-square tests for the communicative function of requesting action

Table 5.10 Results for Chi-square tests for the communicative function of requesting information.....

Table 5.11 Results for Chi-square tests for the communicative function of providing information

Table 5.12 Emails by key participants in Company NZ2

Table 5.13 Main communicative functions of emails in Company NZ2 and Chi-square results

Table 5.14 Results for the Chi-square test for the communicative function of providing information.

Table 5.15 Emails by key participants in Company M

Table 5.16 Main communicative functions of emails in Company $\mathrm{M}$ and Chi-square results

Table 5.17 Results for Chi-square tests for the communicative function of requesting action

Table 5.18 A comparison of main communicative functions of emails in the three workplaces by percentage and Chi-square results

Table 5.19 Results for Chi-square tests for the communicative function of providing information

Table 5.20 Results for Chi-square tests for the communicative function of requesting action

Table 5.21 Results for Chi-square tests for the communicative function of expressing thanks

Table 6.1 Patterns of address forms in Malaysian society (Abdullah, 2003, p. 314)........... 161

Table 6.2 The use of greetings and closings in emails in Company NZ1 ............................ 165

Table 6.3 Types of greeting used in emails in Company NZ1 ......................................... 166 
Table 6.4 Types of closing expressions in email in Company NZ1.

Table 6.5 The use of greetings and closings in emails in Company NZ2 ........................... 171

Table 6.6 Types of greeting in email in Company NZ2 …............................................ 172

Table 6.7 Types of closing expressions in email in Company NZ2 ................................... 173

Table 6.8 The use of greetings and closings in emails in Company $\mathrm{M}$................................ 176

Table 6.9 The use of greetings and closings in emails by CEOs of Company NZ1 and Company $\mathrm{M}$

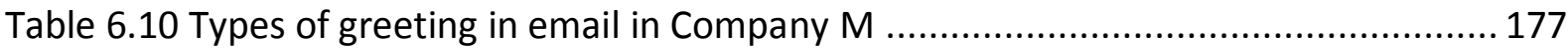

Table 6.11 Types of closing expressions in email in Company M................................... 181

Table 6.12 The use of greetings and closings in emails in Company NZ1, Company NZ2 and

Company $\mathrm{M}$

Table 6.13 Results for the Chi-square test for the use of greeting 184

Table 6.14 Results for the Chi-square test for the use of closing. 184

Table 6.15 Greeting types in Company NZ1, Company NZ2 and Company M...... 186

Table 6.16 Types of closing expressions in Company NZ1, Company NZ2 and Company M. 188

Table 7.1 Summary of distribution of requests realised in imperative, interrogative,

declarative mood and non-clausal units 205

Table 7.2 Distribution of imperative mood and its various forms 207

Table 7.3 Distribution of requests realised in the interrogative and its various forms 211

Table 7.4 Distribution of requests realised in the declarative mood 214

Table 7.5 Position of 'please' in mood clauses..... 219

\section{List of figures}

Figure 2.1 Comparison of polite, politic and appropriate behaviour with respect to marked

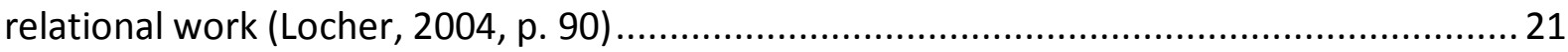

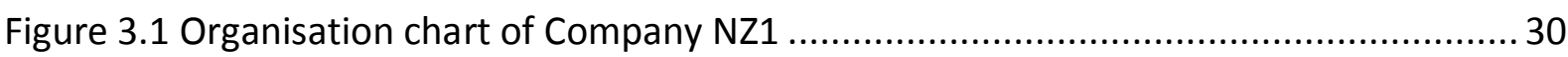

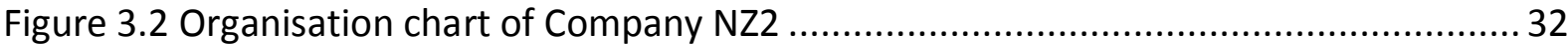

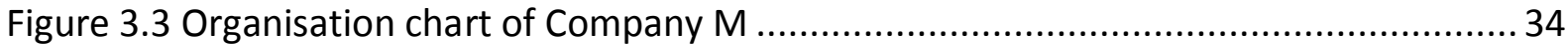

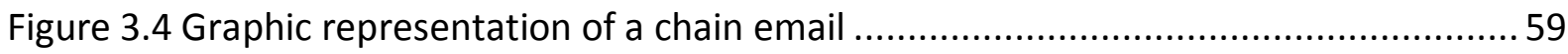

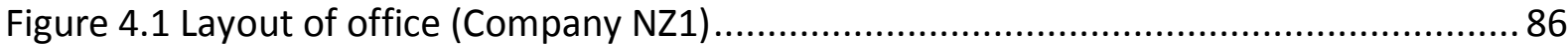

Figure 4.2 First floor of the main administration office building (Company NZ2) ................. 96 
Figure 4.3 Ground floor of the main administration office building (Company NZ2) 97

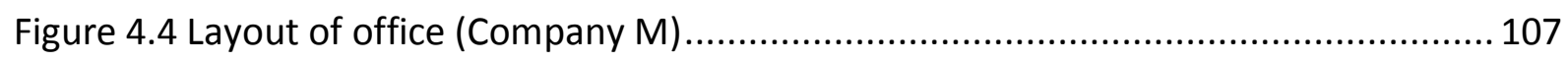

Figure 5.1 Frequency of reported use of email in Company NZ1 ................................... 117

Figure 5.2 Frequency of reported use of email in Company NZ2 ................................... 122

Figure 5.3 Frequency of reported use of email in Company $M$...................................... 126

\author{
List of acronyms \\ CEO $=$ Chief Operating Officer \\ $\mathrm{CMC}=$ Computer-mediated Communication \\ $\mathrm{CMD}=$ Computer-mediated Discourse \\ CofP $=$ Community of Practice \\ HOD $=$ Head of Department \\ $\mathrm{HOE}=$ Head of Events \\ HR = Human Resource \\ $M D=$ Managing Director
}




\section{Chapter 1: Introduction}

The changes in technological invention towards the end of last century have had an impact on the ways we interact with one another in every aspect of life, including in workplaces. The internet has penetrated in almost every aspect of many organisations such as universities (Haugh, 2010, p. 105), businesses (Gains, 1999; Jensen, 2009; Sherblom, 1988) and homes (Li, 2000a). Over the last 20 years, electronic mail (hereafter email) as a tool of communication has developed as one of the main electronic channels of communication. It has made a major impact on many spheres of workplace organisational communication and behavior (Abdullah, 2003; Alsree, 1997; Baron, 1998; Fletcher, 2011; Gains, 1999; Ho, 2009; Kankaanranta, 2005; Markus, 1994a; Waldvogel, 2005).

Email has become a powerful tool and is capable of reaching thousands, even millions of people within and across the globe within a split second. Email has revolutionised workplace communication and has changed the way people communicate at work. A survey carried out in 2012 by a technology market research firm, The Radicati Group reported that the number of email accounts worldwide is projected to increase to 4.3 billion by 2016 from the figure of 3.1 billion in 2011 (Radicati, 2010, 2012). According to this survey, a total of 89 billion business emails are sent and received per day. In a survey conducted in 2011, it was reported that 105 messages are sent and received daily by a typical corporate user. In an earlier survey conducted by the group in 2001,85 percent of participants agreed that email has improved organisational communication (Radicati, 2001).

Email as a means of communication is now firmly established in many organisations and all employees typically have access to it. Many organisations recognise the importance of this medium and have developed their own in-house electronic mail systems to meet the demands of everyday communication (Louhiala-Salminen, 1996). However, this increasing dependency on email has often affected the social relationships between the parties involved. For this reason, a study on the use of email in the workplace and changes in communication patterns is important. It is also crucial to understand the social implications 
of computer-mediated communication (hereafter $\mathrm{CMC}$ ), especially issues relating to interpersonal skills.

This research has been carried out within the Wellington Language in the Workplace Project ${ }^{1}$ (LWP) (Holmes, 2000b). The LWP project, which started in 1996, analyses the way language is used to construct relationships in a wide range of New Zealand workplaces. The LWP has drawn on a varied corpus of mainly spoken data collected in workplaces to analyse characteristics of effective interpersonal communication from a sociolinguistic perspective and to explore the practical implications of the findings to benefit New Zealand workplaces.

Despite the growth of $\mathrm{CMC}$ in workplace interaction, relatively little research has been carried out on workplace email in New Zealand. Two useful studies on the impact of email communication in the workplace in the New Zealand context have been done by two researchers: Joan Waldvogel (2005) and Jeanette Fletcher (2011). Waldvogel looked at the role of email, the style of email communication and gender differences in email use in a white collar versus a blue collar organisation. Fletcher (2011) studied the relational aspect and climate of care in an IT company in New Zealand.

From the cross-cultural perspective, few studies have been conducted on email communication in Malaysia because it is difficult to convince business organisations to share written documents from the workplace. For confidentiality reasons and primarily because emails are now accorded legal status where they are "treated as evidence in a case just as a typed letter or recording of a telephone call" (Mills, Clay, \& Mortensen, 2000, p. 71), it was difficult to obtain authentic emails. To my knowledge, the only studies on the impact of email communication in the workplace in the Malaysian context were done by Abdullah (2003) and Alsree (1997). Abdullah (2003, p. 447) looked at how email can contribute to "a rich repository of relational communication" in a corporate headquarters which had been recently privatised. Alsree's (1997) study focused on evidence of power in email communication in a Malaysian subsidiary of a multi-national corporation whose headquarters was based overseas. I was very fortunate to find a workplace that allowed me

\footnotetext{
${ }^{1}$ See Chapter 3 for more details.
} 
access to the organisation's workplace emails. This research will thus make an important contribution to empirical research on email communication in the Malaysian context.

\subsection{Rationale for the research}

My interest in undertaking a cross-cultural study in New Zealand and Malaysia derived from my own experience and inquisitive nature. I have worked in several large organisations throughout my employment history in Malaysia and have always written emails in a very formal business style. When I decided to undertake PhD research, I started to communicate with my potential supervisor and I was shocked when she asked me to address her with her first name. In my culture where status and title are given emphasis, it is considered disrespectful and rude to address someone who is of a higher status with only the first name. It took me a long time to get used to this style of informal writing because I felt that I was being impolite and disrespectful if I used an informal greeting style. Drawing on my own working experience, I realised that there are many differences in the way New Zealand people and Malaysian people write their emails.

In addition to the informal greeting used in emails by New Zealand people, I also noted other linguistic features which differentiated how the two countries managed email communication. For example, I noticed that Malaysian writers tended to use the imperative mood when making requests of their work colleagues which could be interpreted as rather forceful and sometimes impolite. I had on many occasions received emails which were worded in the imperative mood which I considered rather rude, and I had also heard remarks from my work colleagues that they too had received emails which they felt were quite disrespectful from their peers. Politeness researchers (for example Eelen, 2001; Spencer-Oatey, 2008) argue that no utterance is inherently polite or impolite because according to them politeness is negotiated dynamically among interactants (Eelen, 2001; Spencer-Oatey, 2008). Holmes and Stubbe (2003b) also argue that politeness is context dependent. Accordingly, I started to question whether email communication can do power and build rapport at the same time because of its hybrid features of spoken and written language (Baron, 1998; Crystal, 2006). With these questions in mind, it was natural for me to 
choose a comparative study of workplace email communication in New Zealand and Malaysia.

On the basis of what has been discussed in this section, it is clear that there are opportunities to contribute to research on email communication from a cross-cultural perspective and how it constructs and maintains rapport (Spencer-Oatey, 2000c). The current study was designed to fill these gaps. The aims of the research are discussed in the following section.

\subsection{Purpose of the research}

My research aims to contribute to the research on email communication from a crosscultural perspective by presenting a comparative study of the use of email in three workplaces from two countries with contrasting cultures. This research focuses on authentic threads of internal organizational email messages in order to examine how rapport is developed, as well as to analyse the relevance of power and status in the construction of email messages. The study also examines people's perception of email communication and explores why people sometimes choose email over telephone or face to face interaction. Finally, it also aims to investigate the ways in which interpersonal relations and work relations are negotiated through this mode of communication.

To achieve the aims listed above, the following research questions are addressed:

1. How do managers and subordinates accomplish relational work in email messages, i.e. how is rapport established, maintained and negotiated in email exchanges?

2. How do managers and subordinates construct power and negotiate status-based workplace relationships in email communication? 


\subsection{Structure of the thesis}

This thesis is organised in eight chapters. Chapter 1 relates the background of the study, outlines the rationale and purpose of the research, and proposes two research questions.

Chapter 2 reviews the relevant literature that provides the theoretical frameworks which are drawn on to illuminate the data. It describes the literature on Media Richness Theory (Daft \& Lengel, 1984), CMC as an overarching view and email in particular. Research on politeness theory is reviewed and the study's position on politeness theory (i.e. rapport management (Spencer-Oatey, 2000c)) is explained.

Chapter 3 outlines the methodological and analytical tools which have been used for data collection. Using a mixed-methods approach, the methodological tools include fieldwork observations, questionnaires, interviews and internal work-related emails. These were collected from three workplaces: two workplaces in New Zealand and one workplace in Malaysia. The analysis of emails was carried out in two stages: the first stage involved coding the emails into their various communicative functions; and the second stage involved an indepth interpretation of emails. In addition to Spencer-Oatey's (2000c) rapport theory as the primary theoretical framework to examine the interpersonal metafunction in written communication in more detail, Halliday's three metafunctions were also drawn on to further support the interpretation of the emails.

In Chapter 4, I give an overview of the Community of Practice (hereafter CofP) (Wenger, 1998a) approach as a valuable concept to analyse the linguistic and non-linguistic behaviour of the three contrasting workplaces. Justification for classifying each workplace as a CofP is also provided. Using the CofP approach enabled me to consider how physical spaces can contribute to rapport management (Spencer-Oatey, 2000c) and 'doing' power. I conclude this chapter with a discussion of how new members are accepted as core members in a CofP and the challenges of becoming a member of CofP through the process of legitimate peripheral participation.

The results of the analysis of emails are presented in three chapters - Chapters 5, 6 and 7. In Chapter 5, I present a brief review of Speech Act Theory (Searle, 1969) and the adapted 
version used as a method of categorising emails in this study. Besides providing an explanation of how emails were coded according to their main communicative functions, I also discuss some of the issues related to the coding of the emails and how these issues were addressed. Then a comparison of the main communicative functions between the three workplaces or CofPs is provided.

In Chapter 6, following a literature review on greetings and closings, I compare the use of greetings and closings in the three workplaces. Emphasis is given to how the different types of greetings and closings contribute to the construction of collegiality in the workplace and the impact they have on the instantiation of power.

Chapter 7 presents the results of the analysis of emails, focusing particularly on requests. In this chapter, I identify the different strategies used by managers and subordinates when making requests of each other. This chapter discusses how the various strategies employed by the staff members in emails upward (subordinate to manager), downward (manager to subordinate) and lateral (peer to peer) contribute to rapport building and 'doing' power in workplaces. Rapport theory (Spencer-Oatey, 2000c) form the basis for this analysis and Halliday's (2004) three metafunctions lend additional support to the interpretation of the email messages.

Finally, in Chapter 8, I summarise the findings with respect to the research questions posed in Chapter 1. I also discuss the contributions of this study to the relevant research fields, namely $\mathrm{CMC}$, interpersonal communication and workplace culture research. 


\section{Chapter 2: Literature Review}

\subsection{Introduction}

The objective of this study is to investigate the ways in which workplace relations are negotiated, maintained and enhanced in email communication in three contrasting workplaces. This chapter introduces the theoretical frameworks and relevant previous research studies which are drawn on to illuminate the subsequent analysis. Firstly, the literature on the basic concepts of computer-mediated communication, focussing specifically on email, the notion of a lean medium, and the language of email are reviewed. Discussions of politeness theory complete this chapter.

\subsection{Computer-mediated communication (CMC)}

There is a large literature on $\mathrm{CMC}$, and the number of specific studies on email is increasing. In this section, a brief introduction of the term CMC is given and how and where email is situated is explained.

$\mathrm{CMC}$ has been defined as "a process of human communication via computers, involving people, situated in particular contexts, engaging in processes to shape media for a variety of purposes" (December, 1997, n.p.). In more recent applications, the broad definition of CMC includes both audio-visual media such as interactive video and voice mail and text-based systems such as emails and text messaging. According to Tagg (2012, p. 3), text messaging "does not require [a person] to be sat at a computer" because digital communication can be applied to mobile phones (i.e. smart phones). In other words, the focus of the term CMC has evolved with the advent of mobile communication.

There are two main types of CMC: synchronous and asynchronous. Synchronous or real time $\mathrm{CMC}$ takes the form of simultaneous communication while asynchronous or delayed interactive $\mathrm{CMC}$ means that participants do not communicate at the same time (Maricic, 2005). Each type of CMC has its unique characteristics. For example, writers for asynchronous online systems (particularly email) are less pressured by the time factor when 
composing messages. In contrast, synchronous online messaging systems require the writer to respond instantly. Another feature which differentiates these two types of $\mathrm{CMC}$ is the number of recipients it can address at one time. While both asynchronous and synchronous CMC allow exchanges of messages between a single (dyadic) and multiple recipients (multiparty), some email systems restrict the number of recipients who can be addressed at a single time (Maricic, 2005). Table 2.1 shows the types of synchronous and asynchronous CMC.

Table 2.1 Types of synchronous and and asynchronous CMC (Maricic, 2005, p. 16)

\begin{tabular}{lll}
\hline Sychronous & Asynchronous & \\
\hline Interactive (dyadic/ multiparty) & Interactive (dyadic/ multiparty) & $\begin{array}{l}\text { Non-interactive (monologic/ } \\
\text { broadcast) }\end{array}$ \\
\hline IM (Instant Messaging) & E-mail & $\begin{array}{l}\text { Web pages } \\
\text { ICQ ('I seek you') }\end{array}$ \\
IRC (Internet Realy Chat) & SMS (Short Message Service) & E-journals \\
Social MUDs & Mailing lists & E-zines \\
MOOs $^{3}$ & Usenet newsgroups & E-newspapers \\
2D \& 3D Virtual Worlds & BBS (Bulletin Board System) & E-newsletters \\
Audio-video chat/ conferencing & Computer conferencing & E-advertisements \\
& Interactive blogs & Non-interactive blogs \\
\hline
\end{tabular}

Text-based CMC refers to messages that are typed and projected as text on a computer screen. This can be done by one person or many, depending on the types of messaging system used and the social and cultural context in which they occur. Herring (2001) refers to this type of text-based messages as computer-mediated discourse (CMD hereafter) which is a more specialised term within the general term CMC. Herring (2001, p. 612) defines CMD as "the communication produced when human beings interact with one another by transmitting messages via networked computers". CMD focuses on language and language use in the virtual environment.

Email, which is the focus of the present study, is one example of a text-based CMC and is asynchronous. According to Murray $(1988$, p. 6), email is "the generic term for an asynchronous tool that allows people to type extended messages at computer terminals and

\footnotetext{
${ }^{2}$ Multi-user Dimensions or Multi-user Dungeons, from the early association of MUDs with the role play adventure game Dungeons and Dragons

${ }^{3}$ MUDs, Object Oriented
} 
have those messages electronically transmitted to recipients who can answer, use, or file them. The sender keeps a copy in a computer file".

In the next section, the discussion focuses on email as a text-based CMC or CMD. To avoid confusion, the term $\mathrm{CMC}$ is used to refer to computer-mediated communication in general and email is used when referring specifically to this category of CMC.

\subsubsection{Email as CMC}

A vital tool for business organizations to stay intact in a competitive globalization era is an effective communication system. Since the inception of communicative technologies in the workplace in the late 1970s and early 1980s, email has become one of these new communicative mediums and there have been far-reaching effects on organizational communication and organizational behaviour. A number of studies on email as a medium of communication have been carried out over the past two decades (Abdullah, 2003; Baron, 1998; Brosnan, 2006; Fletcher, 2011; Gains, 1999; Gimenez, 2000; Jensen, 2009; Ku, 1996; Louhiala-Salminen, 1996; Ngwenyama \& Lee, 1997; Rice \& Love, 1987; Sproull \& Kiesler, 1986; Thomson, 2006; Van Mulken \& Van der Meer, 2005; Waldvogel, 2005). These studies show that email has become a substantial part of an employee's working life.

The growth of email has led to a decline in the use of some other modes of communication in the workplace, such as memos, letters, faxes, telephone and face-to-face interactions (Baron, 1998; Long, 1987; Louhiala-Salminen, 1996; Markus, 1994b). Email has slowly replaced the conventional transmission of messages internally and externally in the workplace in the new millennium. Louhiala-Salminen (1996) reported that nine percent of her participants used email as a medium to send English messages and this figure was expected to increase. Another study conducted by Long (1987) reported that about 59 percent of written communication, especially inter-office memos, and about 38 precent of verbal communication, including face-to-face meetings and telephone conversations had been replaced by email. These findings are supported by Markus (1994b) who found that the chairman of the company where she conducted her research encouraged every staff 
member to use email for all work related communication. Baron (1998) reported in her paper that the editor of Microsoft's magazine, Michael Kingley, estimated that 99 percent of Microsoft's internal communication would be via email.

The change in the choice of communication medium has had many negative effects on social and psychological relationships, such as flaming, impoliteness and depersonalisation (Kiesler, Siegel, \& McGuire, 1984; Nadler \& Shestowsky, 2006; Panteli \& Seeley, 2006; Rice \& Love, 1987). Some earlier research focussed specifically on email, argued that email is an impersonal means of communication because it has taken away the human aspects of face to face communication. Email has also removed the social context cues which can influence information exchange. Sproull and Kiesler's (1986, p. 1495) study on emails from a Fortune 500 company shows that "people's feelings of anonymity tend to produce relatively selfcentred and unregulated behaviour" when social cues are weak or absent. They point out that due to the absence of these social cues, email users tended to behave in an unregulated manner which led to negative behaviour such as flaming (i.e profanity, excessive use of capitalizations and exclamation marks). Email users were also said to be "self-absorbed", i.e. the focus is on themselves rather than on others, and they tended to behave more inappropriately online than face-to-face (Sproull \& Kiesler, 1986, p. 1497).

Other researchers note that email creates unnecessary stress (Markus, 1994b; Waldvogel, 1999). Waldvogel's (1999) study of one New Zealand workplace reports that the participants in her research felt that email caused stress because people expected quick responses to email messages. Markus (1994b, p. 142) refers to the 'CYA (cover your a..)' syndrome cited in the questionnaire she administered to 375 respondents of various levels in a risk management company in the US. She reports that there was an obsessive need for documentation when a simple telephone call would have sufficed.

Despite views that email has negative effects, it is still a popular mode of social interaction: it has made communication more efficient and this has increased organisational productivity. Kiesler, Siegel and McGuire (1984) give an example of a firm where a new product idea was posted on the computer network and sent to 300 employees in branches spread across the country. The product was launched after the company received positive feedback within two 
days. Email also eliminates problems such as telephone tag and the social chit chat that occupies much of face-to-face communication (Long, 1987).

In CMC, the social context cues which guide face-to-face interactions are frequently absent and this has affected people's behaviour. For example, social status cues such as job titles, level of hierarchy, age or gender can alter how communication is received or sent. In one study, the results showed that higher level people do not dominate interaction in computer mediated group discussion as they would in face-to-face meetings (Kiesler et al., 1984).

Because of the absence of social cues, email has been considered a lean medium and incapable of transmitting rich information. However, there are alternative views which have been presented more recently and which also need to be considered. In the following section, a discussion of whether email is a lean medium is presented.

\subsubsection{Media Richness Theory}

Email was seen as a lean medium by Daft and Lengel (1984) because it was perceived as lacking intonation and non-verbal cues present in face-to-face communication from the Media Richness Theory (MRT) perspective. This theory, which is also known as information richness theory, was originally proposed by Daft and Lengel (1986) to understand how people make media choices to match their information requirements. According to this theory, the greater the possibility of a message being misinterpreted, the greater the need for rich media. Richness reduces ambiguity and uncertainty, and the choice of media will influence the richness of the messages conveyed. The level of richness a communication medium possesses depends on four criteria: the capacity for immediate feedback; the use of cues such as facial expression, gestures, graphic symbols, tone of voice and numbers; personalisation of the message; and language variety (Daft, Lengel, \& Trevino, 1987).

Sullivan (1995, p. 49) claims that the richness of a channel or its bandwidth refers to "its ability to transmit multiple communication cues, provide instant feedback, and offer a personal focus to the communication". Hiemstra argues:

In general, the research [on the impacts of information technology] points to the conclusion that, as band-width narrows from face-to-face communication interaction 
to computer terminal interaction, the media are seen to be satisfactory for simpler communication tasks, such as information exchange, but less satisfactory for more complex, more personally involving communication tasks [...]. Additionally, as bandwidth narrows, communication is likely to be experienced as less friendly, emotional and personal and more serious, businesslike, depersonalised and task-oriented. (Hiemstra, 1986, p. 206)

Email has been criticised for its incapacity to communicate rich information or foster social relationships and for reducing personal contact between individuals because it is only suitable for single meaning (unequivocal) and task-based informational messages (Markus, 1994a). In a study by Daft, Lengel and Trevino (1987), managers preferred oral interactive media if messages could potentially be misunderstood or when feedback was needed. Email is also said to be impolite and to promote negative behaviour such as flaming (the use of unconstrained, face threatening language in messages) as discussed in the previous section. According to Nadler and Shestowsky (2006), email has taken away certain personal and social cues which are deemed important in face-to-face communication, a view which is also supported by Waldvogel (2001) and Panteli \& Seeley (2006). These studies report that email is seen as being unable to communicate rich information as it lacks body language cues and immediate feedback.

However, the accusation that email is a lean medium has been questioned by researchers such as Markus (1994a), Huang, Watson \& Wei (1998), Abdullah (2003), Waldvogel (2005), Jensen (2009) and Fletcher (2011). Huang, Watson and Wei (1998) show how email has the ability to convey rich information. They suggest that the recipient of the message be given emphasis. Hence rich communication can be achieved because people process the email message by taking into account their own knowledge and relationships involved. When recipients are able to construct meaning from an electronic text, the information is rich.

In the business context, Jensen (2009) concludes that interaction goals can be achieved and long-term business relationships can be fostered via email interactions. Similarly, in the organisational environment, Markus (1994a) observes that effective senior managers were found to use email heavily and even for equivocal tasks. Markus comments that when one wants to avoid confrontation, email is deliberately chosen (1994a). In other words, email is used when unpleasant social interaction needs to be avoided. She uses the example of 
managers who chose to word their messages carefully to maintain interactional relationships. Abdullah (2003) argues that email can convey rich relational communication since writers can personalise their messages. Her research shows that email is capable of doing both affective and transactional work. In addition to its ability to construct relational work as reported by Abdullah (2003), Waldvogel (2005) and Fletcher (2011) argue that email plays an important role in knowledge creation.

From the discussion above, it can be argued that email is capable of producing rich information. In this study, email communication allows the managers and subordinates to combine their own linguistic resources and the technology to personalise their messages to convey relational information and build rapport with each other in transactional messages. These aspects of email communication are explored in Chapters 6 and 7.

Email, one of a number of mediums of communication in workplaces, has been likened to both speech and to writing. While email is clearly a written medium, the flexibility of integrating speech-like features in emails provides the writer with an option to compensate for the absence of social cues in CMC. The next section explores the issue of whether email is speech or writing.

\subsubsection{The language of email}

There seems to be no consensus that email combines the characteristics that are associated with written or spoken language (Baron, 1998; Crystal, 2006; Gains, 1999; Gimenez, 2000; Lan, 2000; Rice, 1997). On the one hand, email can be seen as a form of writing because it can be printed and it is capable of generating a paper trail (Baron, 1998). On the other hand, it is ephemeral and similar to speaking because messages can be deleted by pressing the delete button (Baron, 1998). Rice (1997) notes the feature of a combination of speaking and writing patterns in the 200 emails of four participants from midlevel positions in the four American companies where he conducted his research. He reports that the messages were compact and brief which suggests a blend of informal and formal discourse features. He notes that although email displays interactive elements such as the use of active verbs, simple sentences, and brief paragraphs, there are also features of rigid and traditional conventions of business writing. 
Email has been described as a hybrid language which combines the features of both spoken and written language (Baron, 1998; Ferrara, Brunner, \& Whittemore, 1991; Gains, 1999; Kankaanranta, 2005; Louhiala-Salminen, Charles, \& Kankaanranta, 2005). Kankaanranta's (2005, p. 261) study of 282 internal email messages written in Business English Lingua Franca shows that the discourse in emails which was classified as Dialogue messages (messages exchanging information) exhibited more spoken features than that in the Postman messages (messages delivering other documents or messages) which contained "outdated phraseology" of formal business letters. Dialogue messages exhibited Chafe's notion of "possibility of immediate feedback" and "involvement", characteristics of spoken communication rather than written communication (1982; cited in Kankaanranta, 2005, p. 251).

Referring to email as a hybrid language, Ferrara, Brunner and Whittemore (1991, p. 12) state that email took the form of an amalgam of three language varieties: postcardese, headlinese and telegraphese. Baron (1998) supports this description by attributing the hybrid nature to the schizophrenic-like characteristic of email. Crystal (2006), on the other hand, does not view email as just a hybrid of writing and speech. He regards email language as consisting of "speech + writing + electronically mediated properties" (Crystal, 2006, p. 51). Therefore, he introduces what he claims as a "genuine third medium", that is computer-mediated language called Netspeak in addition to the two existing mediums: written language and spoken language (Crystal, 2006, p. 52).

Baron compares email to other languages and proposed email as a type of Creole:

When the linguistic profile of email is compared with those of other evolving communicative systems, it becomes clear that the seemingly schizophrenic character of email reflects ongoing creolization. Users of email (like users of creolized spoken, signed, or written systems) have considerable choice over how to formulate and respond to messages, stressing the character of one progenitor or another. This variation is evident in the stylistic range found in today's email messages, even when sent by the same individual. (Baron, 1998, p. 164)

Baron (1998, p. 144) also suggests that email mirrors "a moving linguistic target rather than a stable system" and gives three reasons for this suggestion: the evolution of technology from an awkward into a sophisticated system; the diversification and growth in usership, and 
the partial maturation of the process in that users have become more familiar with the system.

As a written medium which incorporates the features of speech, email offers writers the option to be formal as well as to be informal. Waldvogel's (1999) analysis of 50 emails identified a combination of speech and writing elements. Some emails were composed with care while some had missing capitals, a mixture of lower case and upper case letters, spelling and punctuation errors, and the like. She comments that while some of her participants attributed these contrasting stylistic features to email being an informal mode of communication, others felt that a carefully worded email is a reflection of the writer's personality. In other words, depending on the perception of the relationship between the writer and the recipient, email communication allows for a range of speech styles to be used.

Similarly, Ferrara, Brunner and Whittemore's (1991) examine the Interactive Written Discourse (IWD) which consists of stylistic and syntactic features used in business and find a combination of spoken and written features. The spoken language features displayed were the absence of articles, pronoun subjects, contractions or informal spellings (e.g. nite for night), and clippings (e.g. info for information) and finite forms of the verb 'to be', while the written language features exhibited were expanded and elaborated syntax with "frequent use of relative clauses, adverbial clauses, and subordination" (Ferrara et al., 1991, pp. 18-19; 24).

Harrison's (1998) study of listserv email messages shows that email displays features of both written and spoken discourse. Another interesting finding is that email contains features which are the result of the software, such as the insertion of quotations without retyping, a feature not normally found outside email. She also finds that email can do both transactional and interactional work which further supports the notion that email is not a lean medium and suggests that it is capable of communicating non routine tasks.

Gains (1999) and Gimenez (2000) also find that emails exhibit features of both informal and formal language in their research on business emails. Gains (1999) compares email messages from two different environments: academic and commercial, and finds marked differences 
in the discourse features between the two types of email messages. The commercial emails displayed features of the standard written business English generally "used between cooperating colleagues" (Gains, 1999, p. 98). In contrast, the style used in academic emails ranged from semi-formal to informal with some messages displaying "evidence that writers are mimicking a form of conversation, albeit conducted in extended time and with an absent interlocutor" (Gains, 1999, p. 99). Gimenez's (2000) comparative study of 63 business email messages and 40 business letters from the same company suggests that written business communication has been influenced by computer-mediated communication, and notes a more informal and flexible register in the commercial emails.

On the basis of the discussion above, it is clear that email messages display a combination of features of both written and spoken communication, as well as "electronically mediated properties" as noted by Crystal (2006, p. 51). In the present study, the features of written language in emails indicate that email is less formal than traditional conventions of business letters, while the features of spoken language suggest a degree of informality between writer and recipient. The informal characteristics of emails seem to reflect a relaxed and casual style of communication between interactants. Such features are likely to contribute to establishing a closer relationship between the writer and the reader. While the informal style of email helps build rapport, the formal style of email seems to reflect the professional image of the company. In other words, this combination of features of written and spoken communication which is unique to email communication allows email writers to attend to interpersonal relations and construct solidarity while simultaneously getting transactional work done.

As a basis for exploring how email writers show interpersonal relations to others through this combination of features of both written and spoken communication, in the next sections, I give overviews of politeness theory and rapport management.

\subsection{Politeness Theory}

The choice of words and phrases used in any interaction, whether verbal or written, influences how speakers/ writers and addressees perceive their relationships or how they 
would like to be perceived. In order to establish whether email messages carry relational and interpersonal meaning, politeness theory is used. As stated in Chapter 1, this study draws on the constructs of rapport management in the analysis of emails in naturally-occurring interactions in cross-cultural workplace settings. To explain why a rapport management perspective is adopted in this study, standard politeness theory is reviewed and its related issues are explored. Following that is a review of rapport management.

Generally it is agreed that linguistic politeness involves the expression of the interpersonal component of communication. Brown and Levinson's (1987) politeness theory has been used by many researchers (e.g. Alsree, 1997; Morand \& Ocker, 2003; Ting-Toomey \& Kurogi, 1998; Waldvogel, 2005) to study linguistic politeness from a pragmatic point of view. However, although Brown and Levinson's approach to politeness theory remains influential, many researchers have developed this theory further. For example, Holmes (2006) investigates politeness in workplace discourse; Watts (2003), Eelen (2001), and Mills (2003) analyse politeness from a social concept perspective by incorporating social-theoretical elements, while Spencer-Oatey (2000b) takes a culture oriented approach.

In the next section, I provide an overview of politeness theory and a review of rapport management.

\subsubsection{Politeness Theory: An overview}

The initial proponents of politeness theory, Brown \& Levinson (1987), approached politeness from the speaker's perspective and used the concept of the Model Person (autonomous individual). According to Brown \& Levinson (1987), politeness is viewed from how speakers use certain linguistic strategies to show consideration towards their hearers in social interactions. Although their approach has a strong influence in social interactions and has provided an influential framework for many researchers, their theory has received many criticisms (Eelen, 2001; Lakoff, 1973; Leech, 1983; Locher \& Watts, 2005; Mills, 2003; Spencer-Oatey, 2000b; Watts, 2003). First an overview of the theory is given and this is followed by a discussion of criticisms of the theory. 
Brown and Levinson's (1987) politeness theory remains seminal among many politeness researchers because they attempt to provide a universal view on politeness (Eelen, 2001; Terkourafi, 2005). Brown and Levinson's (1987) concept of face derives from Goffman's (1967) notion of face. They define face as "the public self-image that every member wants to claim for himself ... [and assume] that all competent adult members of a society have (and know each other to have) it" (Brown \& Levinson, 1987, p. 61). Later, they take a dual concept of face. They claim that face consists of two specific kinds of desire: negative face, i.e. interactants' desire not to be imposed on and to have freedom of action; and positive face, i.e. interactants' desire to be accepted, liked and understood by others (Brown \& Levinson, 1987, p. 13). Scollon and Scollon (2001) suggest that positive face needs refer to the participants' desire for involvement, while negative face needs are related to the desire of participants to maintain a certain degree of independence. They claimed that in all communications, both positive face and negative face are projected at the same time. Because communication always involves face issues, they argue that interactants must respect face in order to avoid the risk to either independence face or involvement face.

Brown and Levinson's (1987) universal perspective of politeness is based on the assumption that certain types of speech acts, such as disagreements or requests, inherently threaten one's face and they call them face-threatening acts (FTAs). According to them, some acts threaten interactants' negative and positive face and have the potential to cause interactants to lose face when failing to observe interactants' face needs and wants. In order to save face, speakers can choose various strategies from five categories: (1) without redressive action, baldly; (2) positive politeness; (3) negative politeness; (4) off record; (5) don't do the FTA (Brown \& Levinson, 1987, p. 67).

According to Brown and Levinson (1987), the choice of politeness strategy depends on the weightiness of the FTA (Wx). This weight is determined by the value of three factors: (1) the power (P) that a hearer has over a speaker; (2) the social distance (D) between a speaker and a hearer; and ( 3 ) the ranking of impositions ( $R$ ) in a particular culture; it can be represented by the following equation: $W x=D(S, H)+P(H, S)+R x$ (Brown \& Levinson, 1987, p. 76). 
In summary, Brown and Levinson's (1987) theory displays the following characteristics:

a. The focus is on speech acts (FTAs) and it is speaker-oriented.

b. The primary concern is how speakers produce specific linguistic strategies according to a set of universal principles.

c. The theory is aligned toward a normative perspective on politeness.

This theory has been widely critcised. One of the main criticisms is that it does not take into account cultural differences (e.g. Haugh, 2003, 2004; Ide, 1989; Matsumoto, 1988; SpencerOatey, 2000b; Wierzbicka, 2003). Matsumoto (1988) claims that Brown and Levinson adopted a Western bias model. She argues that the theory draws on an autonomous individual without any consideration of other cultures. She says that "a person's self-image in Japan is not as an independent individual but as a group member having certain relations to others" (Matsumoto, 1988, p. 423). Another criticism relates to three factors that determine the FTA strength: P (power), D (distance) and R (imposition). For example, Spencer-Oatey's (1993) study of Chinese and British perception of tutor-postgraduate student relationship demonstrates that Chinese postgraduates show deference to their tutors in terms of respectfulness but expect a friendly social relation, compared to the British students. Brown and Levinson's (1987) politeness theory has also been challenged for giving undue prominence to speech acts which lack discourse level consideration (Eelen, 2001; Mills, 2003; Spencer-Oatey, 2000c; Watts, 2003). Both Eelen (2001) and Haugh (2007) recognise the need to move away from the analysis at the utterance-level proposed by Brown and Levinson because politeness must be analysed as an interactive social practice (rather than as a normative system which is prescriptive) because interactions are dynamic and not static. Moreover, the theory's emphasis on face-work rather than politeness has been widely critiqued (Haugh, 2007; Locher \& Watts, 2005; Watts, 2003). Haugh (2007) suggests an approach which focuses on how politeness is discursively negotiated through interpersonal interaction. Locher and Watts (2005) argue that the study of politeness should go beyond face-threatening act mitigation in order to explain the norms that determine polite behaviour in communication. In addition, Brown and Levinson's theory does not account for the hearer rather emphasising a speaker-oriented approach (Mills, 2003; Watts, 2003). Another criticism observed is that "politeness [...] arises in interaction between 
persons" (Arundale, 2006, p. 194). The analysis in this thesis takes these criticisms into account as well as selecting a framework which takes cross-cultural aspects into consideration.

To overcome these criticisms, politeness theory has been modified and developed. SpencerOatey (2000a, 2000b, 2000c) introduced Rapport theory to take account of politeness considerations in different cultures and her framework emphasises interpersonal relations to address issues concerning the relationship between speakers and hearers. In the next section, Rapport Management is discussed.

\subsubsection{Rapport Management}

To address the criticism of the lack of consideration of cultural aspects of face, SpencerOatey (2000b) proposed a framework that enables researchers to interpret politeness within its interactional context and to take into account different cultures. She also claims that her approach is "more hearer-centred" (Spencer-Oatey, 2005b, p. 335).

Adopting Brown and Levinson's (1987) original aspects of face, politeness research has mainly concentrated on the implications of speech acts that are deemed face-threatening or face-enhancing in interactions. To bridge the gaps in politeness theory, the term relational management has been introduced (Spencer-Oatey, 2000b, 2008; Spencer-Oatey \& Xing, 2003). In explaining relational management, Spencer-Oatey $(2008$, p. 1) argues that when people communicate, it "involves the management of social relations". In other words, when people interact with each other, they are managing and maintaining interpersonal rapport.

This idea of relational work was taken up by Locher and Watts (2005) when they developed their framework. Locher and Watts use the term relational work for this concept; they refer to it as "the 'work' individuals invest in negotiating relationships with others" (Locher \& Watts, 2005, p. 10). In this context, relational work can be understood as falling within Halliday's (1973) interpersonal function rather than ideational function (Locher \& Watts, 2005). The term relational work is based on the notion of frame (Tannen, 1993) and habitus, a term devised by the French sociologist, Bourdieu (1990). A frame is defined as "structures of expectation based on past experience" (Tannen, 1993, p. 53) and the term habitus refers 
to "the set of dispositions to act in certain ways, which generates cognitive and bodily practices in the individual" (Watts, 2003, p. 149). Locher (2004) recognises that relational work does not always mean social harmony and equilibrium. Therefore, the concept of relational work is a broader term for politeness as it includes not just polite or impolite behaviour (Brown \& Levinson, 1987), it also covers social behaviour which is considered appropriate/ inappropriate, politic/ non-politic (Watts, 2003) or marked/ unmarked. Politic behaviour refers to "behaviour, linguistic and non-linguistic, which the participants construct as being appropriate to the ongoing social interaction" (Watts, 2003, p. 21). In contrast, polite behaviour is "behaviour beyond what is perceived to be appropriate to the ongoing social interaction" (Watts, 2003, p. 21). In other words, relational work covers "the entire continuum from polite and appropriate to impolite and inappropriate behaviour" (Locher, 2004 , p. 51). Figure 2.1 shows relational work in the form of a continuum (Locher, 2004, p. 90).

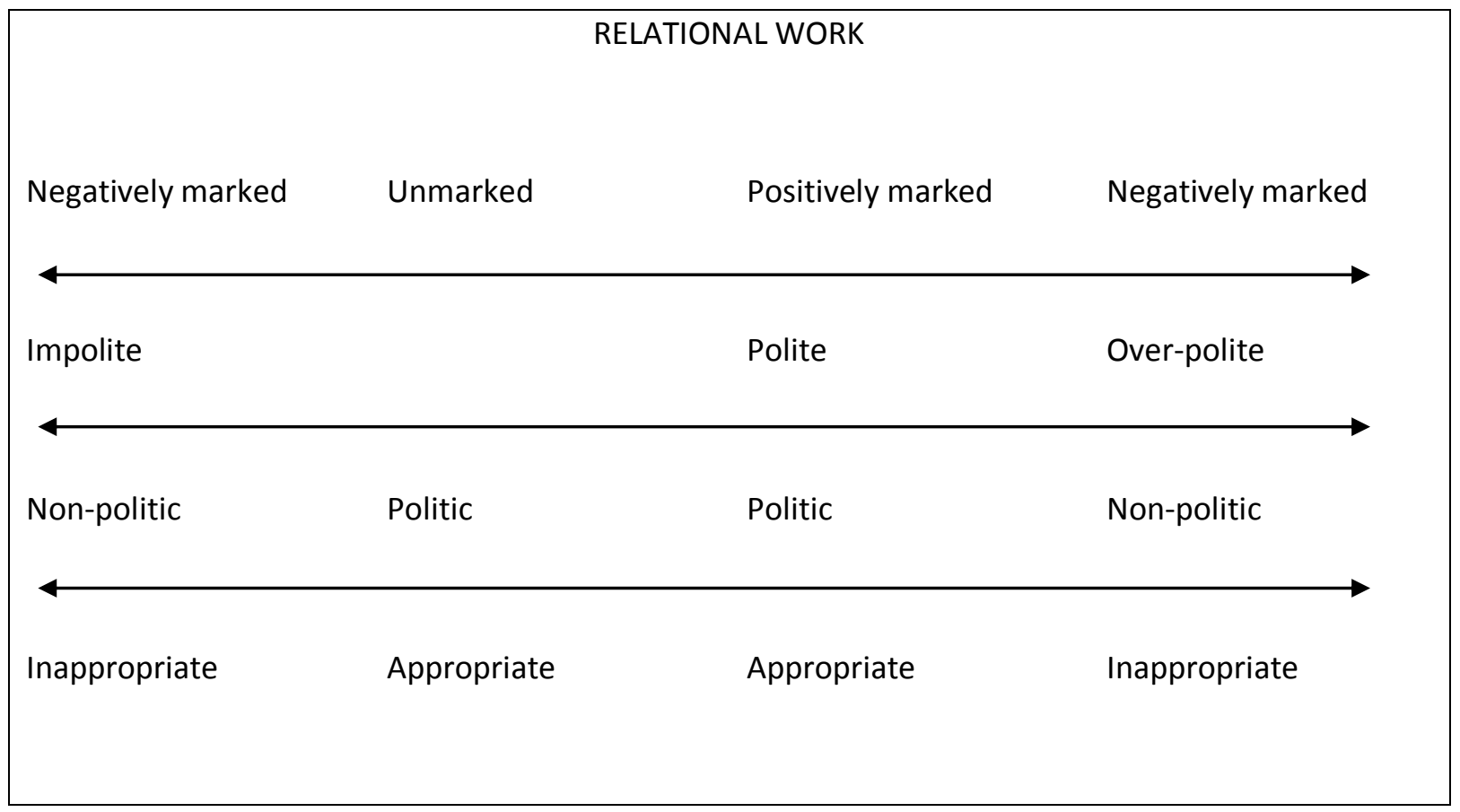

Figure 2.1 Comparison of polite, politic and appropriate behaviour with respect to marked relational work (Locher, 2004, p. 90)

The figure shows that in an interaction where a norm has been established, most relational work occurs in column 2 which is unmarked (i.e. politic and appropriate). Politeness is seen as marked behaviour and displays positive relational work. At the same time it is also politic 
and appropriate (see column 3). By contrast, negatively marked behaviour displays bothover-polite and impolite, non-politic and inappropriate qualities (see columns 1 and 4). Locher (2004) contends that whether something is interpreted as polite or impolite, appropriate or inappropriate depends entirely on the hearer's perception. In addition, politeness cannot be investigated in a vacuum. It must be analysed by looking at "the context, the speaker, the situation and the evoked norms" (Locher, 2004, p. 91). And as Holmes (1995), Mills (2003),Watts (2003) and Locher (2004) point out, no utterance can be considered inherently polite.

Spencer-Oatey $(2008$, p. 3) refers to her framework for analysing politeness in interaction as rapport and rapport management rather than politeness to describe "the use of language to promote, maintain or threaten harmonious social relations". She uses the term rapport to refer to "people's subjective perceptions of (dis)harmony, smoothness-turbulence and warmth-antagonism in interpersonal relations", while the term rapport management refers to "the ways in which this (dis)harmony is (dis)managed" (Spencer-Oatey \& Franklin, 2009, p. 102). She focuses on the illocutionary domain of interaction, an aspect of communication that deals with face-threatening or face-enhancing implications of speech acts rather than Brown and Levinson's (1987) original FTAs. She argues that rapport management is based on the idea that interactants "dynamically co-construct their discourse" (Spencer-Oatey, 2008, p. 6). In other words, it refers to the management of harmony-disharmony among people (Spencer-Oatey \& Franklin, 2009). It covers a wider scope than Brown and Levinson's (1987) politeness theory which focuses mainly on face management. It also suggests an aspect which is missing from Brown and Levinson's politeness theory and that is an aspect which creates more of a balance between self and other, achieved through the management of both face and sociality rights.

Within this framework, Spencer-Oatey (2008, p. 13) claims that "the concerns they [Brown and Levinson] identify as negative face issues are not necessarily face concerns at all" because an utterance can be considered polite and appropriate in one situation but rude and inappropriate in another (Watts, 2003). She also claims that face issues are dependent on a range of attributes associated with them (Spencer-Oatey, 2007). She gives an example of her personal experience with a Hungarian student at a railway station. When the young student complimented her for her kindness using the utterance "Thank you very much. You 
are a very kind old lady.", her positive face was maintained (Spencer-Oatey, 2007, p. 645). However, she also felt a threat to her face at the same time because she saw herself as middle-aged rather than old.

She proposes that rapport management is made up of three components: face sensitivities, sociality rights and interactional goals. The first component, face sensitivities, concerns the personal relational social value, a sense of worth and dignity and reputation of oneself as an individual as well as an individual as a member of a group. It is defined as "the positive social value a person claims for himself [sic] by the line others assume he has taken during a particular contact" (Spencer-Oatey, 2000b, p. 14). This component is further divided into quality face (a desire for a positive evaluation in terms of personal qualities, i.e. positive face in Brown and Levinson) and identity face (a desire for positive acknowledgement in terms of social roles).

The second component, sociality rights and obligations, concerns social experiences. People develop a sense of perceived rights and obligations such as their own entitlement from a legal/ contractual point of view or what is expected from certain behavioural conventions. This component is subdivided into two inter-related aspects: equity rights and association rights, which Spencer-Oatey (2008) labels as socio-pragmatic interactional principles (SIPs). SIPs are defined as "socio-culturally-based principles, scalar in nature, that guide or influence people's productive and interpretive use of language" (Spencer-Oatey \& Jiang, 2003, p. 1635). Equity rights concern the notion of fair treatment while association rights concern the belief that we are entitled to social involvement with others. In other words, we have the right to be able to participate in social interactions and conversations. When these SIPs are not met, interactional rapport can be affected.

The third component, interactional goals, concerns relational and transactional goals. When these goals are not met, interactants can become annoyed and frustrated. Within the rapport management framework, all three components (face sensitivities, sociality rights and obligations and interactional goals) are inter-related. By combining the three components, the focus will encompass the other and not just self (speaker or in the present study, the author of the email). 
According to this model, threatening behaviour can take place in two ways: face-threatening behaviour and rights-threatening behaviour. Some behaviour can cause the interactants to lose face, quality face, but do not violate their sociality rights. So, it is important to make a distinction between the two components (face sensitivities and sociality rights and obligations) because they account for personal aspects of interaction (quality face and equity rights) as well as the social aspect of interaction (identity face and association rights). In the context of the present study, this framework enables the researcher to look into the personal aspect as well as the social aspect of interaction.

Table 2.2 summarises how the notions proposed by Spencer-Oatey (2000c, p. 15) are related to those of Brown and Levinson (1987). The table also indicates that Spencer-Oatey (2000c) broadens the attention on individual to a larger context, to that of the society, and this extension covers a wider concept of face by incorporating the notion of identity face and the introduction of the notion of sociality rights.

Table 2.2 Components of Rapport Management (Spencer-Oatey, 2000c, p. 15)

\begin{tabular}{lll}
\hline & $\begin{array}{l}\text { Face management } \\
\text { (personal/ social value) }\end{array}$ & $\begin{array}{l}\text { Sociality rights management } \\
\text { (personal/ social value) }\end{array}$ \\
\hline $\begin{array}{l}\text { Personal / independent } \\
\text { perspective }\end{array}$ & $\begin{array}{l}\text { Quality face } \\
\text { (cf. Brown and Levinson's } \\
\text { positive face) }\end{array}$ & $\begin{array}{l}\text { Equity rights } \\
\text { (cf. Brown and Levinson's } \\
\text { negative face) }\end{array}$ \\
\hline $\begin{array}{l}\text { Social/ interdependent } \\
\text { perspective }\end{array}$ & Identity face & Association rights \\
\hline
\end{tabular}

This framework extends to include other domains. Spencer-Oatey (2008) suggests that rapport can be threatened in three main ways: through face-threatening behaviour, through rights-threatening/ obligation-omission behaviour and through goal-threatening behaviour. Rapport enhancement and rapport threat are subjective as they depend on the content of the message, people's interpretation of the message and the context in which the message is delivered. In other words, what is perceived by one person as polite may not be viewed in the same way by another person. According to Spencer-Oatey and Xing (2003), politeness is managed through a number of interrelated domains of language use rather than just the illocutionary domain (speech acts). Spencer-Oatey (2000c) argues that rapport is managed across five inter-related domains: (1) Illocutionary domain: this concerns the rapport- 
threatening/ rapport-enhancing implications of performing speech acts such as requests, apologies, etc.; (2) Discourse domain: this concerns the content and structure of an interaction, which include topic management and topic choice; (3) Stylistic domain: this concerns the stylistic aspects of an interchange, such as choice of tone, genre, syntax and use of terms of address and honorifics (e.g. greetings and closing expressions); (4) Participation domain: this concerns procedural aspects of an interchange such as turn taking, inclusion/ exclusion of a person in a conversation; and (5) Non-verbal domain: this concerns the non-verbal aspects of an interchange such as gestures, eye contacts and body movements.

By taking into account these domains, the framework takes into consideration the other rather than just the speaker. It also expands the analysis to a discourse level, rather than remaining at the utterance level. In order to manage interpersonal rapport, Spencer-Oatey (2008, p. 32) suggests four types of rapport orientation that speakers hold:

(1) rapport enhancement orientation - a desire to strengthen or enhance harmonious relations between the interlocutors;

(2) rapport maintenance orientation - a desire to maintain or protect harmonious relations between the interlocutors;

(3) rapport neglect orientation - a lack of concern or interest in the quality of relations between the interlocutors; and

(4) rapport challenge orientation - a desire to challenge or impair harmonious relations between the interlocutors.

Spencer-Oatey (2008) views rapport enhancement and rapport threat as subjective, i.e. dependent on context and people's interpretation. She shares the same opinion as Matsumoto (1988) who considers that the use of language can have an effect on rapport. He draws examples from honorifics, formulaic expression and the verbs of giving and receiving to show how Japanese language and culture can affect social relations between the speaker and the addressee(s). For example, using the formulaic expression "Musume o doozo yorosiku onegaisimasu" (English translation: "I ask you to please treat/ take care of my daughter well") indicated that the speaker shows deference to the addressee (Matsumoto, 1988, p. 409). 
While maintaining the core idea underlying politeness theory, i.e. that politeness is a universal concept that governs interactions as interactants have face needs to attend to, Spencer-Oatey (2000b) makes some improvements to the theory. She argues that no utterance is in itself, inherently polite or impolite and that politeness is a "social judgement" (Spencer-Oatey, 2000b, p. 3). An utterance is judged for its politeness in relation to the context in which it occurs.

She also claims that politeness is "a question of appropriateness" which depends on cultural differences in ways of managing rapport (Spencer-Oatey, 2000b, p. 3). In other words, appropriateness is influenced by culture. Culture is defined as "a fuzzy set of attitudes, beliefs, behavioural conventions, and basic assumptions and values that are shared by a group of people, and that influence each member's behaviour and each member's interpretations of the 'meaning' of other people's behaviour" (Spencer-Oatey, 2000a, p. 4). This definition encompasses some important features of culture: (1) culture is manifested through different types of regularities, some of which are more explicit than others; (2) culture is associated with social groups, but no two individuals within a group share exactly the same cultural characteristics; (3) culture affects peoples' behaviour and interpretations of behaviour; and (4) culture is acquired and/or constructed through interaction with others. Many researchers have adopted Spencer-Oatey's (2000b) theory to analyse cross-cultural communications. For example, Tanaka et al. (2008) investigates Japanese and English apologies, Planken (2005) compares professional and aspiring negotiators in lingua franca sales negotiations and Spencer-Oatey and Xing (2008) look at the impact of intercultural differences during meetings between British and Chinese. Planken (2005) concludes that the use of safe talk, an important aspect of rapport management, occurred only in the initial and closing stages of negotiation. She also concludes that the professional negotiators' engagement in "professional safe talk" in the central bargaining phase served to reinforce their professional identity and develop transactional goals, which can be interpreted as a source of rapport building between the negotiators.

Spencer-Oatey and Xing's $(2000,2003,2008)$ studies concern face issues of a delegation of Chinese business men visiting an engineering company in Britain. The visit was planned as part of an exercise to get to know the business better after successfully signing a deal. This 
visit turned out to be complicated and both parties were unhappy. This caused a rift in the relationship between the two counterparts. Several reasons accounted for this failed relationship: firstly, the Chinese delegation cancelled all the pre-arranged training sessions and demanded sight-seeing and shopping activities for the entire 10-day trip which annoyed the British; secondly, the Chinese felt that they were looked down upon when they were not given a chance to give an introduction speech but the British did not think that a return speech was necessary; and lastly, the Chinese were also unhappy with the seating arrangement at the welcome meeting because they felt that the British were "commanding, in control, contemptuous" (Spencer-Oatey \& Xing, 2000, p. 275). But the British felt that seating at the head of the table was for practical reasons due to the size of the meeting room. In other words, in the Chinese business men's eyes, role rights and obligations and power were handled inappropriately by the British. This example shows that the Chinese delegation felt that their identity face (status and importance) was being threatened by their British hosts with regards to the seating arrangement in the meeting and speech making. Beside face as a factor which contributed to the dissatisfaction, the Chinese delegation also felt that their obligation rights had not been attended to when their expectations were not met. The authors (2000) suggest that the discontentment and dissatisfaction can arise from both parties' lack of understanding of each other's culture and notions of politeness.

Spencer-Oatey's (2008) theory has also been used by other researchers in CMC contexts focusing particularly on emails (Fletcher, 2011; Ho, 2009). By applying the concepts of interpersonal rapport (face sensitivities; sociality rights and obligations; and interactional goals) in the analysis of emails in an IT company in New Zealand, Fletcher (2011) shows how solidarity can be achieved through more than the illocutionary domain. She shows that a richer interpretation can be obtained if the analysis is expanded from an utterance level to a discourse level. Ho (2009) adopts rapport management theory to analyse how email writers managed and enhanced rapport as well as construct their identities in email requests of nonChinese members and Chinese members in an academic institution in Hong Kong. His findings show that leaders who frequently used rapport building moves were able to construct solidarity with their subordinates.

In summary, although Spencer-Oatey's (2000c) theory uses the notion of face which is a core component of Brown and Levinson's (1987) theory, she adds the notion of sociality rights to 
account for social concerns rather than focusing only on individuals. She also maintains that rapport is managed across five interconnected domains including speech acts (i.e. the illocutionary domain). By incorporating the domains, she extends her framework by taking into account the discourse level rather than focusing on speech acts alone. The data from the present study support this framework, in that rapport is clearly managed through multiple domains, particularly the illocutionary and stylistic domains.

\subsubsection{Summary}

So far in the previous sections, standard politeness theory, i.e. Brown and Levinson's (1987) theory, and rapport management (Spencer-Oatey, 2000c) have been reviewed. In the workplace, language is used to perform both transactional goals and maintain relational goals. Therefore, Spencer-Oatey's (2000b) rapport framework has been chosen for this study. Applying her framework to the analysis of workplace emails shows that rapport is managed through a range of discourse domains rather than just the illocutionary domain, and that doing politeness involves more than attending to face sensitivities. In addition, this framework allows not only a micro analysis (discourse level) but also a macro analysis (context level) necessary for a comparative study of New Zealand and Malaysian workplaces.

\subsection{Conclusion}

The present study, which focuses on workplace email, looks at how it is used in three contrasting workplaces and how the authors of emails attend to both the ideational as well as the interpersonal aspects of their communication. It addresses the specific research question 'How do managers and subordinates accomplish relational work in email messages, i.e. how is rapport established, maintained and negotiated in email exchanges?' by investigating the different email styles and discourse patterns used by the participants in the three workplaces. In this chapter the unique characteristics of email as an asynchronous CMC and the guiding concepts that have informed the theoretical decisions made for the present study have been discussed. The methodology adopted in this study is described in the next chapter. 


\section{Chapter 3: Methodology}

\subsection{Introduction}

This chapter gives an account of the methodology adopted for this study and discusses issues of validity and reliability. I provide a detailed explanation of the methodology used to identify the data for investigation and give an account of the participants, methods of data collection, analysis and limitations of this research.

It was not an easy task to identify a suitable research design for my study. To my knowledge, there is no established framework for the study of authentic email communication. To this end, I adopted a mixed-methods approach which involved several data collection procedures: fieldwork observations, questionnaires, interviews and discourse analysis of emails. In addition, I drew on some aspects of ethnography because I found them useful as a method of observing relevant contexts of interaction and collecting background information to support the interpretations and analyses of the emails in my dataset.

I begin this chapter with a description of the workplaces which were the settings for this study. The data gathering processes adopted from the Language in the Workplace (hereafter LWP) project are described, followed by an account of how access was gained to each workplace and how emails were collected from the participants. A discussion of methods of analysis concludes this chapter.

\subsection{Research context}

\subsubsection{Introduction to the workplaces}

This study was set in three contrasting workplaces: a small cohesive organisation and a large international corporation, both located in New Zealand and a medium sized company in Malaysia. In the following three paragraphs, I give a brief introduction to the three workplaces. The names of companies and participants are pseudonyms to protect their identities. 
The first workplace, Company NZ1, a small cohesive company involved with event management, was a non-profit organisation heavily funded by private and corporate sources. This company, located in the centre of a busy suburb in Wellington, occupied half of the first level in a main building. At the commencement of the research study, Company NZ1 had only fourteen people but had a new addition one month after I started my project. It was predominantly female (87\%) and comprised mainly New Zealand European staff. The formal hierarchy observed by Company NZ1 was relatively horizontal, comprising Andy, the Chief Executive Officer (CEO hereafter) and everyone else from the Corporate Team, the Head of Events (HOE hereafter) Team and the Support Team (see Figure 3.1). The Corporate Team consisted of three members: Susan, the Relationship Manager; Marcus, the Finance Manager and John, the Relationship Manager - Events. The Head of Events Team was made up of four members - Lesley, Jacinta, Jean and Symone. There were six support staff members in the Support Team - Annette, Rose, Joline, Teresa, Pat and Sally. At the time of data collection, the company had recently been restructured and following this, everyone reported directly to the CEO. The CEO had labelled the original organisation structure as 'silo' because one support staff reported to one HOE and each event worked independently of each other.

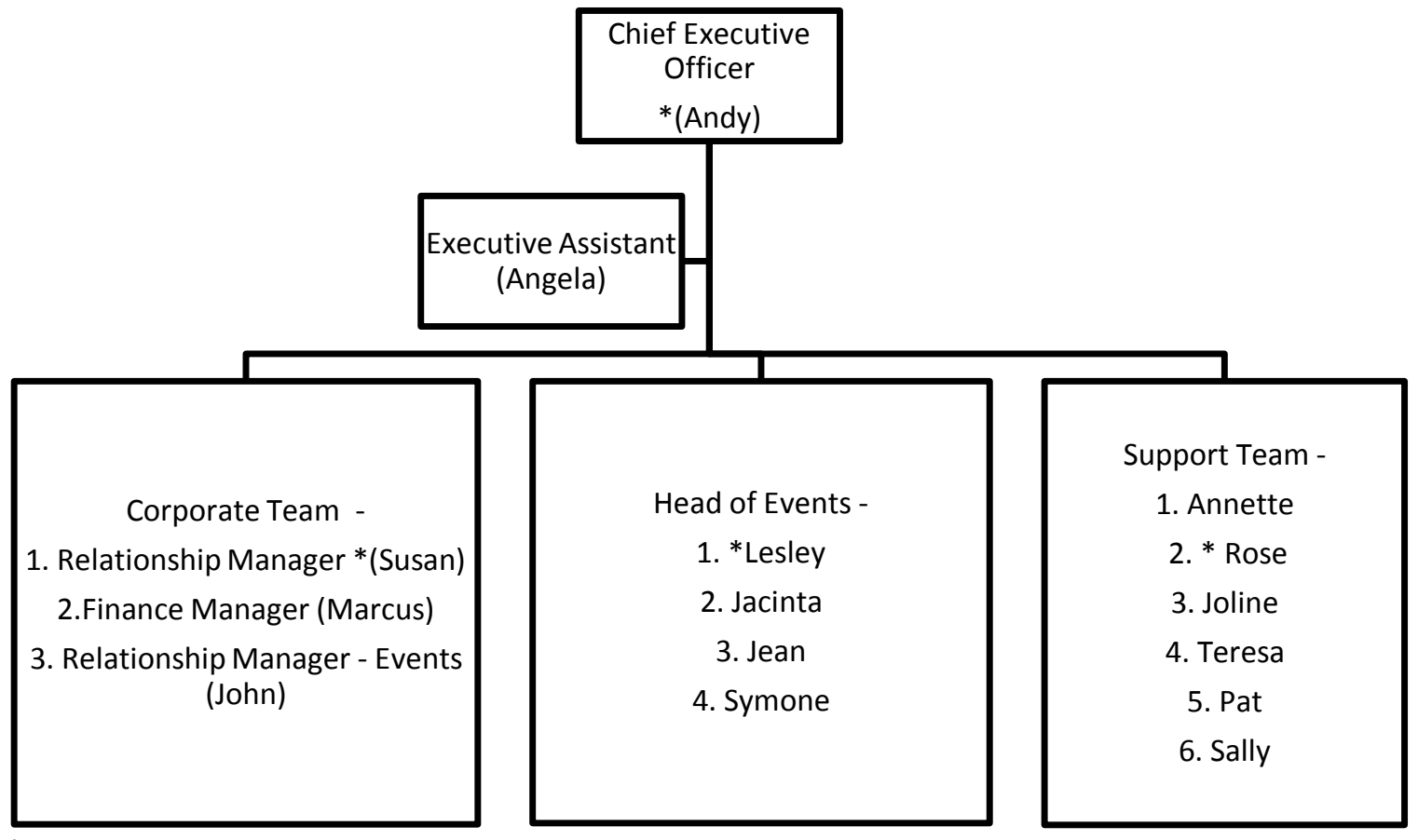

* Key participants are marked with an asterisk

Figure 3.1 Organisation chart of Company NZ1 
The second workplace, Company NZ2, was a large construction company based in Wellington. This organisation was part of a larger international corporation and provided employment for about 200 employees in the Wellington region. The company operated from two branches in the Wellington region and had two divisions: operations and administration. The organisation chart of the branch in which I conducted my research indicates that the hierarchy of the participants of this research had six levels (see Figure 3.2). The General Manager at the top level oversaw both second level divisions which were overseen by an Operations Manager and a Finance Manager. The Finance Manager had three staff subordinates: an Assistant Accountant, a Senior Administrator and an Administrator. The Operations division was a bigger group and had more levels with two Divisional Managers and other managers reporting directly to the Operations Manager. Because one of the key participants (Patrick) belonged to the Maintenance section in the Operations division, I will use this division to explain the level of hierarchy and reporting line that this workplace observed. In the Maintenance section, the Divisional Manager reported directly to the Operations Manager with a Senior Manager at the fourth level reporting to the Divisional Manager. The Senior Manager had three subordinates who were engineers. At the fifth level, one of the engineers who held the role of a Manager had the following subordinates: a team of blue collar workers and an Assistant Manager. Company NZ2 was a predominantly male organisation (90\%) and it comprised mostly New Zealand European staff with a minority of other ethnicities. 


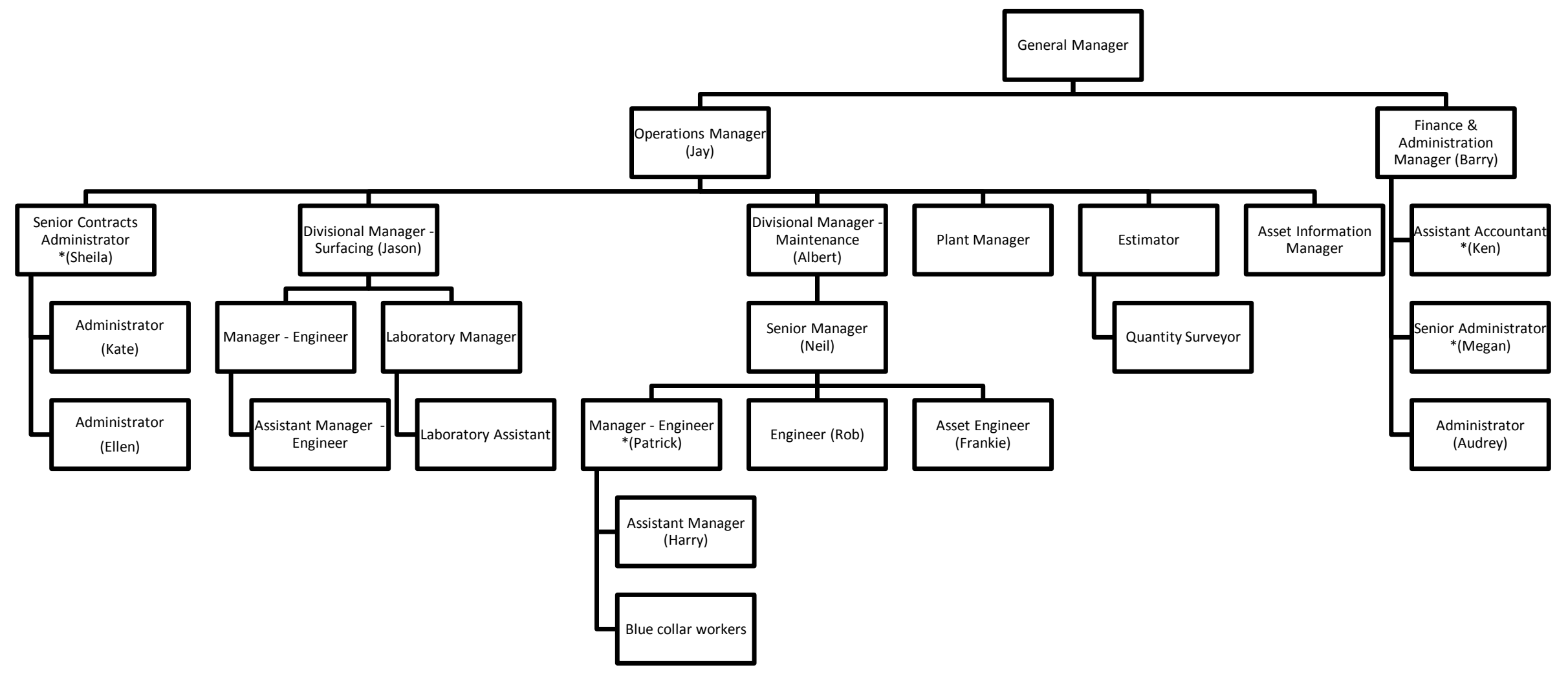

*Key participants are marked with an asterisk

Figure 3.2 Organisation chart of Company NZ2 
The third workplace, a Malaysian company, Company M, was a firm of Chartered Accountants which offered a wide range of business solutions. The company was formed as a partnership before it was incorporated as a private limited company in the 1990s. The company, which celebrated its tenth anniversary in 2011, had a strong clientele ranging from engineering, construction, manufacturing, health and pharmacy sectors. Under three different company names, it offered a wide range of services such as internal auditing, tax, corporate finance, corporate insolvency/ recovery, secretarial and accounting, with the main focus on auditing. At the highest level, there was a Board of Directors which consisted of the Managing Director (hereafter MD) and the Chairman of the company (see Figure 3.3). Next in the hierarchy was the CEO who oversaw the day-to-day business operations of the company. All Heads of Departments, the Human Resource (hereafter HR) Manager and the IT Manager reported directly to the CEO. They were responsible for their respective departments and their immediate subordinates. For the present study, I worked with only two departments: the Corporate Governance and Human Resource Departments, therefore the description of the organisation chart is limited to these two departments. The Head of Department (hereafter HOD) and the HR Manager each had three and four staff members reporting to him and her respectively. 


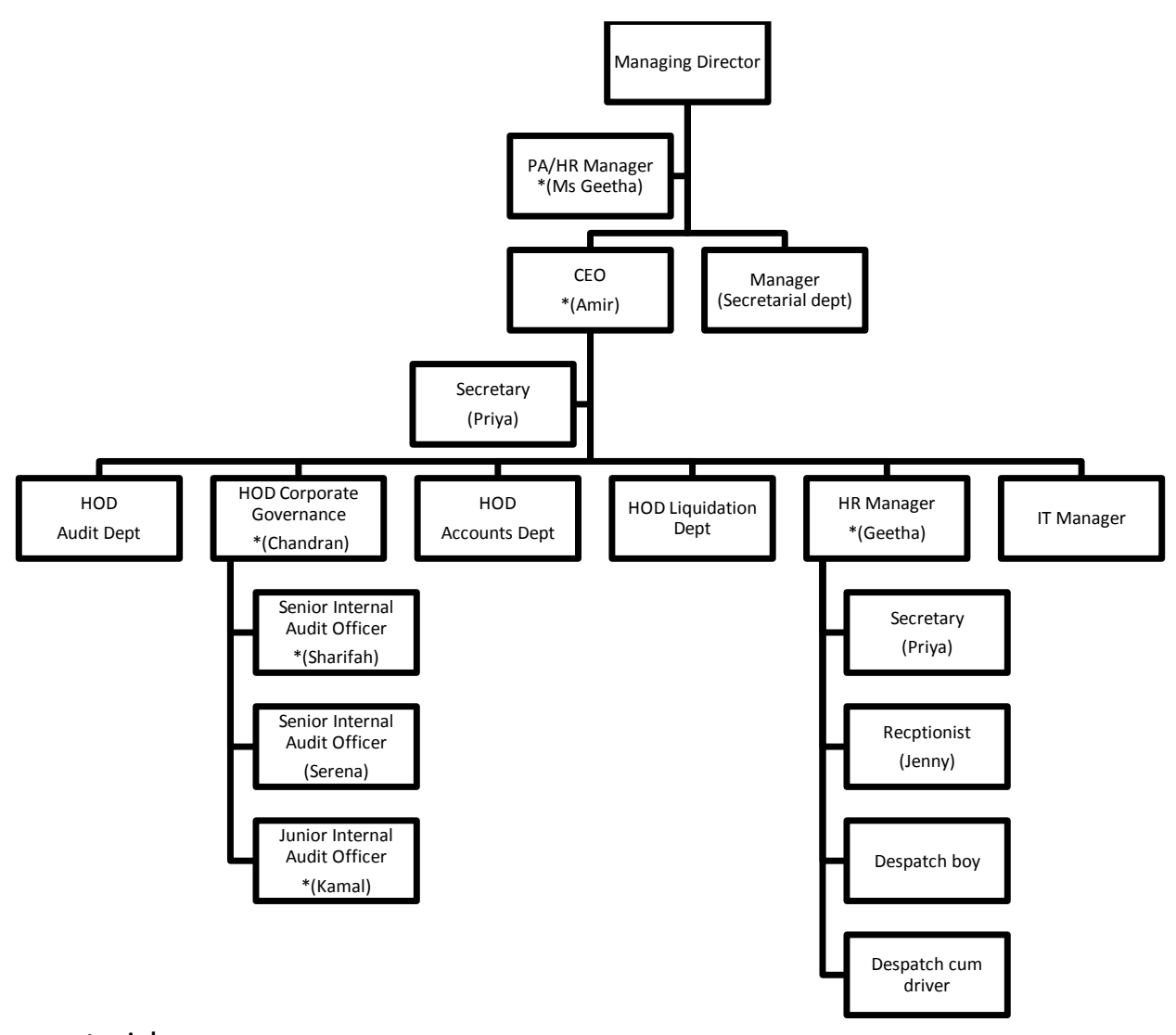

*Key participants are marked with an asterisk

Figure 3.3 Organisation chart of Company M 


\subsubsection{Research participants}

To address the research questions, I planned to collect data from four key participants in each workplace who would participate in the research by providing me with internal workrelated emails for analysis and by being interviewed about their email practices.

The CEO of the first workplace (Company NZ1) gave permission to carry out the research and everyone in that organisation volunteered to participate. As a result, I collected emails from all 15 participants and interviewed all the staff members. For my study, one participant was selected at random from each team. So, the key participants were the CEO, the Relationship Manager from the Corporate Team, one HOE from the HOEs Team and one Support Officer from the Support Team.

In contrast to the first workplace, at the second workplace (Company NZ2), the Divisional Manager whom I approached to obtain permission to conduct my research nominated four staff members who agreed to participate. The four staff members were my key participants and they were from different levels of hierarchy - a manager who was also an engineer, a senior administrator from the operations division, an assistant accountant and a senior administrator from the administration division. Internal emails were collected from these four key participants and I interviewed a total of seventeen employees in this workplace.

To obtain a comparable dataset, I informed the CEO at Company $M$ that I would like to analyse emails written by staff members from different levels of hierarchy in the company. As with the second workplace, the CEO nominated three staff members from the Corporate Governance division and the HR Manager to be my key participants in the study. The key participants were the CEO, the HOD, the HR Manager, a senior internal audit officer and a junior internal audit officer. 
Tables 3.1, 3.2 and 3.3 summarise the key participants in each workplace.

Table 3.1 Key participants in Company NZ1

\begin{tabular}{ll}
\hline Name (pseudonym) & Role in the organisation \\
\hline Andy & CEO \\
\hline Susan & Relationship Manager \\
\hline Lesley & HOE \\
\hline Rose & Support Officer \\
\hline
\end{tabular}

Table 3.2 Key participants in Company NZ2

\begin{tabular}{ll}
\hline Name (pseudonym) & Role in the organisation \\
\hline Sheila & Senior Administrator - Operations \\
\hline Patrick & Manager - Engineer \\
\hline Ken & Assistant Manager \\
\hline Megan & Senior Administrator - Administration \\
\hline
\end{tabular}

Table 3.3 Key participants in Company M

\begin{tabular}{ll}
\hline Name (pseudonym) & Role in the organisation \\
\hline Amir & CEO \\
\hline Chandran & HOD \\
\hline Geetha & HR Manager \\
\hline Sharifah & Senior Audit Officer \\
\hline Kamal & Junior Audit Officer \\
\hline
\end{tabular}

\subsubsection{Ethical considerations}

I gained ethics approval (see Appendix 1) from the VUW Human Ethics Committee prior to the commencement of my fieldwork. Consent forms and information sheets were designed in which it was made explicit that participants were given the right to withdraw from the project at any point up to two months after data were provided and any emails donated and interview recordings of them would be deleted. None of the participants from the workplaces withdrew from the project.

The key consideration regarding ethics was confidentiality. Participants were given the assurance that pseudonyms would be adopted to preserve confidentiality and none of the participants or the organisation would be traceable. Assurance was also given that only the 
researcher and the supervisors would have access to the materials collected and that these materials would be locked away safely. Recordings of interviews were kept locked securely in the computer which would require my password in order to get access. Transcriptions of interviews and completed questionnaires were properly labelled, filed in a ring file and locked in a metal cabinet for safekeeping. As the materials collected for my research are emails, i.e. written documents, it was a matter of trust that I would delete any emails which were not intended for my research or when I was requested to do so by the participants. I received only one request on this issue. The CEO from Company NZ1 requested that the emails which were forwarded automatically via the Rules and Alerts feature for one day be deleted. She informed me that the emails contained some confidential staffing issues, and I deleted them immediately. See Section 3.4.4 for details of the Rules and Alerts feature.

Ethical issues were discussed in the first meeting with the CEO of Company NZ1 and the Divisional Manager of Company NZ2 and they were also addressed during meetings with the potential participants. An information sheet covering the nature and length of the research, storage of data, protection of the organisation and participants' identities was distributed and discussed. For Company $\mathrm{M}$, a different approach was taken because I was unable to go to Malaysia for a face-to-face meeting. I contacted the MD via email and a proposal outlining this research including the information about the aims and methods of study, the data collection process, ethics and confidentiality and the costs and benefits of the research to the company were sent to the company prior to his agreement.

In line with the LWP project's basic principle of giving as much control as possible to participants (see Section 3.3.1), people were also given the option to select the emails that they would like to donate to the researcher for the purpose of this study. Opportunities to ask questions were given to the participants before their written consent was collected. For Company $\mathrm{M}$, all queries were addressed via email through the CEO, who was my main contact person. Since all the participants in the three companies had given their consent, all internal emails were used except emails relating to sensitive issues such as staff members' salaries and other confidential matters.

As noted in Chapter 1, it was very difficult to convince business organisations to share written documents in the workplace. To express my appreciation to the participants who 
participated in my research, I gave them morning tea treats and souvenirs on the last day of my observation.

\subsection{Research design}

\subsubsection{Language in the Workplace (LWP) ${ }^{4}$ project}

This research study adopted a participatory approach, an approach similar to that used by the LWP project team at Victoria University of Wellington. The LWP project began in 1996 and was spearheaded by Professor Janet Holmes of Victoria University of Wellington to explore effective workplace communication in various settings (e.g. hospital, factory, government). Some of the areas of workplace discourse based on authentic workplace interaction include leadership discourse (Holmes, Marra, \& Vine, 2011; Schnurr \& Chan, 2009), business meetings (Chan, 2005; Marra, 2003; Murata, 2011), organizational knowledge creation (Fletcher, 2011), workplace email communication (Waldvogel, 2005) and directives (Vine, 2001).

The LWP team has developed a distinctive methodology for collecting interactions for workplace discourse in New Zealand (see Holmes \& Stubbe, 2003b; Marra, 2008). In terms of the data collection procedures, volunteers in each organisation were asked to record their own work-related interactions with two aims in mind (1) "to give participants as much control as possible over the research process, and especially the data collection process; and (2) to reduce the researchers' involvement in the physical collection of data to the absolute minimum" (Holmes \& Stubbe, 2003b, p. 21). Some examples of studies which made use of this model or a similar model are Murata (2011), Fletcher (2011), Chan (2005), Marra (2003) and Alsree (1997).

The stages of the LWP data collection process are:

1. Initial meeting with the management

2. Volunteers record their own data

3. Feedback sessions

4. Data analysis

\footnotetext{
${ }^{4}$ Details of LWP are also available at this website: http://www.victoria.ac.nz/lals/lwp/.
} 
I have only adopted those aspects of the methodology which were relevant for my study. I give a detailed account of each stage in the next section. I explain how access was negotiated and how the initial meeting with the management of the three workplaces was arranged.

\subsubsection{Gaining access}

One of the central problems researchers face is gaining access to the field (Watt \& Jones, 2010). As it is difficult to collect authentic data in business settings (acknowledged by Bargiela-Chiappini \& Harris, 1997), identifying key people, i.e. "gatekeepers" (Denscombe, 2010 , p. 89 ) is crucial to the success of gaining entry to the research site. In addition researchers have to work with whichever companies agree to participate in the research project.

Researchers have to be prepared to face challenges if they choose to be involved in authentic workplace research. In my case, the disappointment of being turned down by several workplaces. My first experience was with Company $A$, the first workplace which we ( $m y$ supervisor and I) approached at the end of 2010. The representative from Company $A$ had shown great enthusiasm and had agreed to participate in my research project and plans were made to meet the team at the beginning of 2011 , however, two days before the arranged meeting, Company A withdrew from my research. With this rejection, we started to look for another workplace. My second experience was another workplace which also showed great interest in my research project, but which decided to postpone their involvement to a later time because of work commitments and priorities.

My subsequent experiences involved rejections from a number of workplaces in Malaysia. Generally, it was very difficult to find workplaces in Malaysia that were willing to participate in this study as people are reluctant to share their written documents. An email was sent to one workplace which showed some interest but it was rejected when the Deputy Vice Chancellor of the workplace declined to give consent explaining "Unless there is a formal collaboration with us in the form of joint research, we are unable to consider supporting request of such nature." 
After failing to secure approval from the President and Chief Operating Officer to conduct research at my previous workplace, I wrote to the Vice Chancellor. Unfortunately, again, my proposal was turned down due to issues of confidentiality.

While rejection is something that is unavoidable in authentic workplace research, there are also other workplaces which are supportive of research of this nature. Following examples by practitioners of participant observation such as Agar (1996) and Holmes (2000b) who sought personal contacts through networking, I was fortunate to get my first research participant, Company NZ1, as my entry point to the 'real' research world which began in March 2011. The selection of the second and the third workplace relied on social networks or 'friend-of-a-friend' approach (Milroy, 1987). The social network established through attending a local church in 2010 to 2011 assisted in making contact with the participant in Company NZ2 (the second workplace) and the third workplace in Malaysia (Company M) was secured through a personal contact. Access to these three workplaces is discussed in Section

\subsection{3.}

As cautioned by Watt and Jones (2010) negotiating entry into the workplace proved to be the most difficult phase in my research since it was the workplaces, i.e. the gatekeepers (Denscombe, 2010), that chose to give their cooperation. Negotiating entry had to be done cautiously and carefully to protect the good name of the University. Although there were numerous rejections encountered throughout the whole negotiation period, such situations must be handled in a diplomatic way.

\subsubsection{Initial meeting with management}

\subsubsection{Company NZ1}

Entry to Company NZ1 was first negotiated by my supervisor, Professor Janet Holmes, with the Relationship Manager via email. An appointment was set with the CEO on the $1^{\text {st }}$ March 2011. At our initial meeting we discussed the objectives of the research and the types of data that I aimed to collect. At this initial meeting I provided a written information sheet which outlined the project (see Appendix 2), a proposal outlining the LWP project (see Appendix 3) as well as the consent form (see Appendix 4) for the participants (Marra 2003, Waldvogel 2005). The CEO expressed her interest in participating in the research and gave us 
an overview of the changing culture since her appointment the previous year. After this discussion, the CEO invited us to give a presentation about my research project to the staff members at the company's weekly meeting the following week.

During this presentation, I was introduced to the staff members of Company NZ1. The CEO was very positive but stressed that there was no pressure on anyone to participate. A copy of the LWP proposal, information sheet, consent form and questionnaire (see Appendix 5) were distributed to all staff members. All who were present returned the consent forms immediately. One staff member collected consent forms for two members who were not present at the meeting. They completed the forms and returned them to me within a week. It was agreed that I take on some tasks during my time there. Data gathering was arranged for one full day each week but to obtain rich observational data, I spent at least three full days in a week in the organisation in the first month. I also chose to go on different days of the week for two and a half months to carry out my observation to enable me to see how all the staff members interacted with each other as not all of them worked every day.

\subsubsection{Company NZ2}

For the second workplace, a church friend who worked in this large New Zealand organisation asked the Divisional Manager to meet me and arranged the initial meeting opportunity in August 2011. I met the Divisional Manager and talked about my research project, explaining it using the research information sheet for participants and the LWP initial proposal. After the Divisional Manager obtained approval from the Operations Manager of the Wellington branch, a meeting was set up to meet with the four nominated key participants to discuss the detailed arrangements for the project. This meeting gave my supervisor and me the opportunity to address any issues that the key participants had before they gave their consent to participate in my project. One participant enquired about the benefit of conducting research of this nature while the rest had no comment. As with the arrangement with Company NZ1, it was also agreed that I take on some administrative tasks during my time in Company NZ2. It was decided that I start the following week. Due to the nature of the business, I went to 'work' as early as six in the morning in order to capture all the actions taking place. I also chose to go every day for three weeks as it gave me the opportunity to become familiar with the staff members in this large organisation. 


\subsubsection{Company M}

The third workplace was secured with the help of a close friend who had vouched for my credibility as a researcher and this gave me an opportunity to present my proposal to the MD. Because I was in New Zealand during this negotiation period, a different approach was adopted and all communication was done via email. After obtaining permission from the MD, the next level was to get an agreement from the CEO who was quick to agree and gave me full cooperation. I informed him of the criteria for choosing key participants for this project and three weeks later, he gave me the nominated staff members. Unlike the other workplaces where I had the opportunity to meet the participants before the project began, in Company $\mathrm{M}$, all queries were addressed via email. The only concern the company had was the issue of confidentiality and the assurance of giving pseudonyms to both participants and company was well received. As with the arrangement with Company NZ1 and Company NZ2, it was agreed that I take on some administrative tasks during the time I was at the workplace to conduct observations. Unlike the first two companies where I started immediately upon obtaining consent from the "gatekeepers" (Denscombe, 2010, p. 89), my first day at the workplace was five months later, i.e. May 2012.

It is important to note that, as in many applied linguistics research studies, my study uses non-probability sampling (i.e. convenience sampling). According to Wagner (2010, p. 25), convenience sampling "involves surveying individuals who are readily available and that the research has access to". In other words, I worked with workplaces which were "available" (Dornyei, 2007, p. 129). While I cannot make generalisations on the basis of the three workplaces because each workplace was different from the others, my data analysis show that features of email communication in one of the New Zealand workplaces was more similar to that of the Malaysian workplace in a number of respects than it was to the other New Zealand workplace. See Chapters 4, 5, 6 and 7 for details.

\subsubsection{Preparation for data collection}

Before I entered the 'real world' to collect data, I trialled the proposed methods to test their suitability. The trial period provided me with valuable information which helped me improve on the methods before I adopted them in the actual research sites. In the sections 
that follow, I discuss each method by outlining how it was trialled, the challenges faced, the final decisions made and the actual data collection.

\subsection{Data Collection}

The main aim of my research was to collect the following data (presented in the order they were collected) from the workplaces ${ }^{5}$ :

1. Observation notes detailing the artefacts in the workplace and behavioural patterns of the participants.

2. Workplace email questionnaire to survey people's use of emails, their reported email practices as well as their attitudes towards the use of email in the workplace.

3. Interviews to collect the participants' reflections about their email practices and their reactions to the email messages they received. The interviews were also used to find out people's use of various communication modes, their rationale for the choice of media, as well as providing a follow up to the questionnaire.

4. A dataset of email exchanges between the participants on various topics in order to analyse how email was used, what it was used for, and whether other modes of communication affected the use of email. The emails also provided material for an analysis of stylistic features.

The data collection methods described above allowed for a degree of triangulation. Triangulation refers to the use of more than one approach to address the problem of subjective interpretation in qualitative research and ensure that the data collected is credible (Denzin, 1989). Webb and his colleagues $(1966$, p. 3) suggest that "once a proposition has been confirmed by two or more independent measurement processes, the uncertainty of its interpretation is greatly reduced". More specifically, according to Duff (2006), readers should be able to make their own evaluations based on the different types of data presented to support the findings of the research. In my study, credibility has been addressed through the different methods employed to triangulate data analysis questionnaires, interviews and observation field notes (see Section 3.5).

\footnotetext{
${ }^{5}$ For the Malaysia workplace, steps 3 and 4 were reversed.
} 
The following sections give a description of each data collection method and the trialling process that the researcher experienced. First I explain the trialling process and then the actual data collection procedure. The basic methodology for collecting data from the Malaysian workplace followed the standard process applied in the two workplaces in New Zealand as closely as possible to ensure comparability (Holmes \& Stubbe, 2003b). While ensuring that this standard process adopted by LWP researchers was followed closely when collecting data in Malaysia, some adaptation was implemented where necessary. This is explained in the sections that follow.

\subsubsection{Observation/ Field notes}

Observation is crucial for gaining an understanding of the organisation as an interactional setting. It also provided an interpersonal and transactional setting for the nature of the email which enabled me to understand how, when and why the emails were written. Hymes (1974) states that it is also important to pay attention to details such as the physical layout of the workplace, location and other artefacts. To do this, I drew on some aspects of ethnography which are explained in Section 3.4.1.1.

In order for me to be able to collect useful and rich data from the actual data collection site, I undertook a trial study to collect some basic information, to determine the feasibility of collecting a useful dataset to answer my research questions as well as to learn the ropes of ethnographic observation. The pilot study was conducted over a two day period in a university department office in December 2010. The following lessons learned during this trial process proved valuable and were used as benchmarks when I went to the actual research site to conduct my observation:

- I needed to be a member of the staff in the office so that my presence at the workplace would not be awkward. For this I suggested that I was given a chore to help out in the daily operation of the office while taking down observation notes.

- In order to follow the events of what happened in the office, I needed to be there for a period of time. Three to five days was judged a good time frame to get to know more of the office culture and to follow the events that took place in the office. In other words, it is important to immerse oneself in the culture if one needs to obtain rich data and understand what is taking place (Hymes, 1974). This concurs with many 
researchers' experiences of immersing oneself within the cultural setting (Hammersley, 2007; Lofland \& Lofland, 1995; Watt \& Jones, 2010).

- It is important to know the names of the people and what they do. I found that it was easy for me to take down my observation notes if I knew the names of the people in department.

- It was also important to know the structure of the organization as it helped to understand why things were done in certain ways or why people reacted in a certain manner.

The important lessons I learned from the trial study were applied when I carried out the fieldwork observations in the three workplaces. According to Nunan (1992), a researcher in a qualitative study can be both a participant and observer or just an observer, and for this study, I assumed the role of both observer-as-participant and participant-as-observer. In the first workplace I spent a period of two and a half months (March to mid May 2011) and a total of about 150 hours taking on the two roles mentioned above. In the second workplace, I spent about three weeks and a total of 82 hours of observation. The field notes were collected from end of August till the middle of September 2011. In the third workplace, the observation period was from $8^{\text {th }}$ to $19^{\text {th }}$ May 2012, and I spent eight days and a total of 50 hours to collect field notes. Positioning myself at a strategic location provided me with an opportunity for observation (see Section 3.4.1.1). I did not look for specific behaviours and my observation was to gather a general sense of how people went about their daily routine and to develop a feel of what it was like to be part of the organisation. This method enabled me to gather specific examples of types of interaction that occurred during the working day to support my subsequent analysis of emails. Joining the staff members for morning tea birthday celebrations (Company NZ1 and Company NZ2) and lunch (Company NZ1 and Company $\mathrm{M}$ ), enabled me to observe their behaviour outside the office. Overall, the purpose of observation was to understand cultural patterns and contexts, as discussed in Section

\subsubsection{1.}

Field notes taken during the observation periods are a useful tool as they are a record of the past that is retained in the present. According Emerson et al. (2001, p. 353) field notes are descriptive writing that "embodies and reflects particular purposes and commitments, and it 
also involves active processes of interpretation and sense-making". They can be recorded electronically or hand written. As I was not given a workstation in the office, I kept a hand written record of my observations in a notebook. Notes were written up on the spot when I was able to or as soon as possible later in the day (Palmer, 2010). These notes were usually written as narrative accounts which 'captured' what I saw and observed. However, sometimes they were just brief notes as a snapshot of what I observed at that time. At the end of each work day I reviewed my notes and this helped me to develop an overview of the organisation. Besides taking notes about the layout of the office, artefacts and decoration of each workspace, I made time-coded notes, in the course of a normal working day, about what was happening in the office. This allowed me to notice patterns in the on-going activities.

\subsubsection{Aspects of ethnography}

The observation/field note practices I used drew on some aspects of ethnography, which I discuss in this section. Sociolinguistics has drawn on ethnography, which is rooted in anthropology, to develop a qualitative participatory approach which provides observational tools and techniques to collect data in the real world as opposed to laboratory conditions. Research which takes an ethnographic perspective uses direct observation to study people in their own settings to obtain rich data about the people and their culture, and in my research the people are employees at their workplaces. Conducting research in specific workplaces provides insights into the social contexts, shared repertoires and membership of group members (Holmes, 2005; Schnurr, 2009). The ethnographic perspective is important in gathering authentic linguistic data as well as in helping the researcher to better understand the target community.

Willis (2000, p. viii) writes that ethnography is the "eye of the needle through which the threads of the imagination must pass". He points out that ethnographers must report the experience and activities observed in detail with evidence and not through imagination. His assertion supports Hammersley and Atkinson's (2007) argument that observers should report accurately what is observed not just contextually, but culturally, socially and politically. Observers must record what is seen and should not be biased with their own interpretations and perceptions. In order to gather rich data, ethnographers must immerse 
themselves within the cultural setting (Hammersley \& Atkinson, 2007; Jones, 2010; Lofland \& Lofland, 1995; Watt \& Jones, 2010).

Research which uses an ethnographic approach aims to provide detailed descriptions of events and cultures. The goal of ethnography is to "elicit insiders (emic) perspectives as well as those of the researcher, undertaking participant observation (etic) perspectives' (Duff, 2010 , p. 52). In other words, an ethnographic approach assists the researcher "to grasp the native's point of view" (Denscombe, 2010, p. 81).

Hammersley and Atkinson (2007) argue that ethnography is not just gathering information; it is about changing understanding. In ethnographic research, an observer's paradox (the possibility that participants may behave differently when under observation) should not be viewed as a problem (Feagin, 2013). Instead researchers develop an awareness of possible observer effects and ask relevant questions to address the limitations. According to Frey, Botan, Friedman and Kreps (1991, p. 231), "ethnography emphasizes studying subjective reality over objective fact-finding". Using this approach, patterns are identified and hypotheses are developed after the data have been collected (Frey et al., 1991).

One benefit of taking an ethnographic perspective is that it provides a holistic view. Denscombe cites Malinowski's view (1992, p. xvi) of ethnography as:

One of the first conditions of acceptable Ethnographic work certainly is that it should deal with the totality of all social, cultural and psychological aspects of the community, for they are so interwoven that not one can be understood without taking into consideration all the others. (Denscombe, 2010, p. 80)

Thus, the ethnographer must take into consideration not only the individual's or group's behaviour under investigation but also the contexts in which the behaviour occurs. In my study, when I was in the office, I joined the staff members for lunch as it was important to preserve the "naturalness of the setting" (Denscombe, 2010, p. 206) and during these times a great deal of useful information was obtained. In addition, I was given rides to the workplace by one of the staff members. During these rides I was given more 'insider' information and was able to seek clarification of certain issues that took place in the office when I was not there. The additional information provided further insights to the workplace culture. 
Although I needed to develop a deeper understanding of the workplace culture, I also needed to avoid interfering with the daily routine in the office (Saville-Troike, 2003). For example, at Company NZ1, by situating myself in the centre room in the office, I was able to see when my help was needed and when I should not disturb the staff members. For the first few weeks with the team I restricted my interactions to the Support Team members, but as I got to know the other staff members better, they started to share with me more information about their roles and responsibilities in the organisation.

Conducting research in workplaces involves "the investment of a sufficient amount of time to build trust with respondents, learn the culture to the extent possible and test for misinformation introduced by both the researcher and the researched" (Davis \& Henze, 1998, p. 404). In my study, the time spent in each workplace very much related to what was possible. Although I attempted to undertake as much participant observation as possible, I was bound by the constraints of the workplace. I could only stay for as long as the workplace would tolerate my presence and I could not stay beyond being made welcome. So in each case I spent as long as possible given the workplaces' view about how much time they were prepared to make available to me (see Section 3.4.1 for the duration spent in each workplace).

According to Saville-Troike (2003, p. 89), researchers who study their own culture have the benefit of making "explicit the systems of understanding which are implicit" because they are able to "use themselves as sources of information and interpretation". This selfknowledge and cultural awareness was especially useful when I conducted my observation in Malaysia. Being in the workforce and having worked in Malaysia for more than a decade before I embarked on this PhD journey, I had an advantage because I was able to make "subtle interconnections of meaning in ways that an outsider could attain only with great difficulty" (Saville-Troike, 2003, p. 90). As stated in the previous paragraph, gaining trust is another important feature which needs attention from researchers who take an ethnographic perspective. In the beginning, I took on some simple office tasks such as laminating work, wrapping parcels and making coffee for morning tea. As I spent more time in the office I was given more administrative responsibilities as I gained the participants' trust. Eckert (2000) has demonstrated in her research on high school students in Detroit the importance of a researcher gaining the trust of her participants in order to collect authentic 
interaction data. During my research, the fact that I was given office-related tasks which dealt with clients' information suggested that trust had been earned (Watt \& Jones, 2010).

There are various approaches to ethnography and I make reference to two which investigate the way the target community works with a focus on language, namely, the ethnography of communication (Hymes, 1968) and linguistic ethnography ${ }^{6}$ (Creese, 2010; Rampton, 2007).

The ethnography of communication deals with the patterns of communication and cultural behaviour within a speech community. This discourse approach was first developed by sociolinguist Hymes (1974) who believes that there is a strong relationship between language and culture. The approach is further developed by Saville-Troike (2003, p. 2), who states that the focus of the ethnography of communication is "the speech community, the way communication within it is patterned and organised as systems of communicative events, and the ways in which these interact with all other systems of culture". Speech community, the primary unit in the ethnography of speaking, is defined by Hymes (1974, p. 51) as "a community sharing knowledge of rules for the conduct and interpretation of speech. Such sharing comprises knowledge of at least one form of speech, and knowledge as to its patterns of use. Both conditions are necessary". In other words, the community's communicative practices are studied as a whole and not as a single entity.

The ethnography of communication examines the localised patterns of communication by paying close attention to how and for what purposes language is used in specific contexts. For example, it examines speech events or sequences of speech such as a dialogue between two or more people, a christening of a baby or a lecture within the context in which these events occur. It also takes into account the diversity of communicative practices which exist in human societies, and recognises the fact that these form an integral part of what members of a particular (sub)culture know and do. In other words, the focus in ethnography of communication is the ability of the speakers to communicate appropriately within a particular speech community, and the skills they need to make use of it (Saville-Troike, 2003).

An analysis of communicative or speech events takes into account the following dimensions: the genre or type of event; the topic; the purpose or function of the event; the setting including the location, time of day, season of year, and physical aspects of the situation; the

\footnotetext{
${ }^{6}$ see The Linguistic Ethnography Forum at http://www.ling-ethnog.org.uk/
} 
participants involved with reference to their age, gender, social status, ethnicity, relationship to one another or other relevant categories; the message form; the message content; the sequence of communicative acts; the rules for interaction and the norms of interpretation (Saville-Troike, 2003, p. 110). An ethnographic approach which focuses on these dimensions is useful for any discourse analysis research, and it has proved to be equally useful for my study because it supports the process of interpretation and inference of email communication.

Rampton (2007, p. 585) describes linguistic ethnography as a "site of encounter where a number of established lines of research interacts", which include: new literacy studies; interactional sociolinguistics; critical discourse analysis; interpretive applied linguistics for language teaching; and new-Vygotskian research on language and cognitive development. Linguistic ethnography brings together two fields of study - linguistics and ethnography - to investigate the role language plays in a wider social context. Linguistic ethnography argues that combining the two fields offers great benefits: first, ethnography "provides linguistics with a close reading of context", while linguistics "provides an authoritative analysis of language use" (Creese, 2010, p. 139); and second, using discourse analytic tools, linguistic ethnography focuses on "'topic-oriented' ethnographies of specific types of professional interaction" rather than "comprehensive descriptions of speech communities" (Rampton, 2007 , p. 592). Some studies which adopt this approach to investigate different groups of people in different communicative events are Chinookans (Hymes, 1996), 'jocks' and 'burnouts' (Eckert, 2000) and 'Latina youth gangs' (Mendoza-Denton, 2008). These diverse linguistic ethnographies provide a rich source of information about the authentic language events of the communities in various settings.

Even though my research study was not an ethnography of communication or a linguistic ethnography per se, I drew on the ethnographic principles outlined above when I conducted my observation at the workplaces. These observations and field notes provided useful and rich background information about the workplace cultures and their practices which allowed me to interpret and analyse my email dataset more fully. 


\subsubsection{Questionnaires}

A pen and paper questionnaire adapted from Waldvogel's (2005) study containing four sections and a total of 58 questions (see Appendix 5) was designed to find out how staff members used emails, their attitudes to email and their email practices. Before the research began, I trialled draft versions of the questionnaire with three groups of people who were working in an office environment and who were of similar background to my target participants. Trialling the questionnaire before administering the final questionnaire was a crucial step to ensure the suitability of the questions, avoid any glitches and to help check for researcher bias in the questions. This concurs with Bloomer (2010) who stresses that a trial study is important so that one can identify potential problems and remove them before embarking on the actual research.

I worked through three rounds of trialling the questionnaire; each round was with a different group of people. The first round was to check on the suitability of the questions designed in the questionnaire and its length. I made changes to some words and phrases to reflect an office rather than a factory environment following feedback from the first round of trialling. Then I administered a second round of trialling after reorganising the sections in the questionnaire. For example, the section to elicit demographic information was put at the beginning because participants felt that the questions were easy to answer and it was a good warm up before answering the more serious questions. In the third round, attention was given to the presentation of the questionnaire, and participants were asked to comment on whether the layout of the questions and instructions given were clear. After the third round, I was confident that the final version was suitable. The final version of the questionnaire is made up of four sections:

1. Section A: participants' demographic profile (eight questions);

2. Section B: participants'email usage and the frequency of using email for 13 communicative functions (five questions);

3. Section C: participants' attitudes towards the use of email (five questions); and

4. Section D: participants' email practices (40 questions).

In Company NZ1, all the staff members completed the questionnaire and returned it to the researcher within three weeks. 
In Company NZ2, a set of questionnaires was given to the Divisional Manager on my first initial meeting with him. On my second visit to the company to meet the four key participants, four sets of completed questionnaires were returned. Twenty-five staff members from various departments including two from a different location were approached individually and asked if they were willing to complete the questionnaire. Two staff members declined to complete the questionnaire. Although twenty-five questionnaires were distributed, only seventeen were returned. Therefore in total, I collected twenty-one completed questionnaires from Company NZ2.

As stated earlier, I adopted a different approach with Company M. Because I was in New Zealand at the time permission was granted, I emailed the questionnaire to the CEO and requested him to send it to all his staff members. Thirty-one completed questionnaires were returned. With the help of a friend, the completed questionnaires were collected from the office and posted to me in February 2012.

Table 3.4 shows the breakdown of the roles/ work area of the staff members who completed the questionnaire in each of the three workplaces.

Table 3.4 Breakdown of the roles of staff members in Company NZ1, NZ2 and M

\begin{tabular}{|c|c|c|c|c|c|}
\hline \multicolumn{2}{|l|}{ Company NZ1 } & \multicolumn{2}{|l|}{ Company NZ2 } & \multicolumn{2}{|l|}{ Company M } \\
\hline CEO & 1 & Administrative Manager & 1 & CEO & 1 \\
\hline Executive Assistant & 1 & Divisional Manager & 2 & HODs & 1 \\
\hline Corporate Team & 3 & Engineer & 3 & Manager & \\
\hline HOES & 4 & Manager & 5 & Executive & 3 \\
\hline \multirow[t]{7}{*}{ Support Team } & 6 & Senior Administrator & 2 & IT Consultant & 7 \\
\hline & & Administrator & 2 & Secretary & 1 \\
\hline & & Assistant Manager & 2 & Senior Officer & 1 \\
\hline & & Chief Civil Engineer & 1 & Junior Officer & 6 \\
\hline & & Quantity Surveyor & 1 & Administrative Clerk & 8 \\
\hline & & Laboratory Technician & 1 & & 3 \\
\hline & & Estimator & 1 & & \\
\hline Total: 15 & & Total: $\mathbf{2 1}$ & & Total: 31 & \\
\hline
\end{tabular}




\subsubsection{Interviews}

Two trial interviews were conducted to avoid potential problems such as asking inappropriate questions and interviewing unsuitable participants (Hymes, 1970). The first one was conducted with a staff member of a university department who was similar to my target group of participants, and the second interview was conducted with a PhD colleague. The main purpose of conducting these interviews was for me to check if the prepared questions were easily understood, to improve on the questions if they were ambiguous or unclear, and to see how the other party would react to the questions. Another advantage of the trial session was to check the reliability of the information by asking several people similar questions (Saville-Troike, 1997).

I learned three closely related lessons from the interview practices. First, it is important for an interviewer to ensure that the interviewee is put at ease and does not feel pressured as this is likely to affect the outcome of the interview. Second, I needed to establish rapport with the participants before beginning the interview. This follows Eckert's (2000) approach: she did not approach the students at Belten High school until she was accepted and had built rapport with them. After modifying the interview questions based on the experiences learned from the trial process, interviews were only conducted after I had familiarised myself with the staff members of all the three workplaces and rapport was established. Thirdly, it is a useful strategy to employ open-ended 'share with me your thoughts' and 'tell me about' questions, as suggested by a PhD colleague, to allow participants to present whatever first came to their minds regarding their email practices. According to Angrosino (2007), semi-structured interviews are characterised by a set of pre-prepared questions that are focused on specific domains of interest. The flexibility of semi-structured interviews allows the researcher to explore and relate to other issues as well as to new information revealed during the interviews.

In Company NZ1, all staff members indicated their willingness to be interviewed. Interviews were conducted face to face at the workplace over a period of two weeks between end of March and mid April 2011 and each took between thirty minutes to one hour. The total time spent on interviews was about 14 hours. These one to one interviews were guided with a set of questions which provided a structure for the interview and ensured that all information 
needed was covered. As I had already established rapport with the staff members, the interviews were very relaxed and those whom I interviewed shared their views with me openly and at length, that most of the interviews I conducted exceeded the initial time limit agreed upon.

While the interview sessions in Company NZ1 were long, in contrast, the interview sessions with staff members in Company NZ2 were rather short with the majority of the interview sessions about 15 to 20 minutes long. Only four staff members were very open and happy to share information at length with these interview sessions lasting between 30 to 40 minutes. The total time spent on interviews was six and a half hours and the face-to-face interviews were conducted within two weeks at the end of August 2012. While all the interviews were conducted in the office in Company NZ1, I interviewed one of the key participants in Company NZ2 in the car when he went for site visits. From this experience, I learned to be flexible and to accommodate to staff members' availability. In other words, interviews could be conducted anywhere and at any time.

All interviews in Company M were conducted face-to-face in May 2012 except for one which was conducted via Skype in January 2012. This interview was arranged in advance because Kamal, the key participant, had resigned from the workplace. Interviews in this workplace were similar in length to those in Company NZ2, and the majority of the interview sessions were about 20 minutes due to the participants' busy schedules and work demands. The longest interview session, 52 minutes, was conducted with the CEO who was very open and happy to share information at length. Although the interview sessions were short, I was fortunate that some of the participants interviewed were willing to share more information over lunch. The total time spent on interviews in this workplace was four and a half hours.

Denscombe (2010, p. 182) suggests that it would be easier to contact prospective interviewees in advance but he also cautions that researchers "cannot always control events as they might want". Although I made appointments with the staff members prior to the interviews, I was not able to follow the schedule strictly. Sometimes interview sessions were postponed because of the staff members' ad hoc meetings and other work which had to take priority. Interview sessions which were postponed due to work commitments were arranged at a later time. My regular presence at the workplace enabled me to conduct the 
postponed interviews on an ad-hoc basis. Therefore, I was able to interview all the staff members in Company NZ1. Unfortunately in Company NZ2 I was unable to interview two staff members although they had agreed to be interviewed.

With the staff members' consent, all interviews in the three workplaces were audio recorded. To ensure that the recording was clear, I used a good quality sound recorder, the Olympus VN6800pc model. The recording was not only clear, its sound file, which is WMA, was also compatible with Transcriber 1.5 .1 (copyright 1998), the transcribing software I used to transcribe the interviews. The audio recording did not seem to influence the flow of the interview and the interviewees appeared to be comfortable. However, on one occasion, one staff member in Company NZ2 put on a serious tone when he knew that the session would be recorded, but as the interview progressed he became more comfortable. In another case, an interviewee in Company $\mathrm{M}$ was initially hesitant to share information because the interview was recorded. However, after assuring her of strict confidentiality, she became more at ease and the interview went well. By recording the interview sessions, I was freed from taking notes and I was also able to maintain eye contact with each interviewee, an important aspect in conducting effective interviews (Macksoud, 2010).

The interview sessions were valuable as they assisted me to better understand the context within which the email message exchanges took place and they provided valuable historical information to aid my interpretation of the meaning of the messages. The interviews were also especially useful to clarify missing data in the questionnaire. By interviewing each staff member, I was able to obtain a more complete understanding of the issues relating to their individual communication practices and styles.

\subsubsection{Emails}

There were various valuable lessons learned during the trialling period. One of the practical considerations which arose was how to collect emails from the participants with minimal interruption to their work. After an initial discussion with my supervisor and with the help of one of my PhD colleagues, a Rules and Alerts feature (hereafter the Rule) was designed. This feature which automatically forwarded all sent emails to my email account was offered to the participants. This option was to save the staff members' time and trouble of having to 
remember to forward the emails to me manually. However, the participants were also given the option of manually selecting their choice of emails to be forwarded to me, a principle observed by LWP researchers, i.e. giving participants as much control as possible over the research process (Holmes \& Stubbe, 2003b). In what follows, I briefly describe the procedure used to collect emails from the participants in the three workplaces and this is followed by the explanation how the emails were labelled.

The Rule was offered to all the staff members in the first workplace, Company NZ1. Staff members who dealt with sensitive and confidential issues were requested to select and manually forward emails to me. In order to test that the Rule worked properly before offering the CEO this option, I sought help from two staff members, Rose and Joline, whom I had, by this time, developed a very good rapport with. As a start I emailed them the Rule and gave them specific instructions on how to import it into their Outlook programme. There were some initial teething problems which were later solved with the help of the PhD colleague who helped design the Rule. Three staff members declined to use this option because they dealt with sensitive and confidential issues. One week after the Rule was imported in the CEO's Outlook programme, she removed it as she had some confidential issues to deal with. Emails from the CEO were then sent to me manually on a daily basis.

I received all kinds of emails - ranging from new emails, replies to emails, meeting invitations, meeting cancellation notices and setting appointments to forwarded emails from the staff members. In total I received 923 emails from all the 15 staff members who donated the emails. Apart from a few deleted email messages which were confidential in nature and contained messages from external clients, all internal work related emails were collected over a period of two months. However, for the purpose of the present study only emails donated by the four key participants were used for analysis. The total number of emails from the four key participants was 611 . Fifty-five percent of the messages were first or primary communications on a particular subject.

Email was a common way of forwarding on images, documents and contact details to each other. Most of these emails followed face to face conversations and did not have any subject lines. Only a small percentage of the emails were on-going dialogues. As these emails were automatically forwarded on to me via the Rule feature and they provided me with the 
background information of some of the workplace issues, I was able to understand some of the on-going discussions when I was in the office.

In Company NZ2, all the key participants were offered the Rule. However, only one accepted the offer while the other three participants opted to forward their emails to me manually. Participants who chose to forward their emails to me manually did so at the end of each week for a month. One of the problems encountered when participants chose to forward the emails to me manually was that they had to remember to do so and it took precious time to manually select the emails. As a consequence there was a risk of not receiving enough emails from the participants. For example, after one and a half weeks, I received only thirty emails from Megan. Fortunately, because I was conducting observation at the workplace, I was able to remind her to forward more emails to me. Another problem encountered with emails which were forwarded to me manually was that I had to discard some because they contained messages from external clients. After one month, I received a total of 575 emails from the four key participants. Sixty-seven percent of these messages were first or primary messages on a topic.

In contrast with Company NZ1 and Company NZ2, the Rule was not offered to Company M. Instead, the key participants of Company M were requested to select 100 emails from their inboxes to be forwarded to me and all attachments in the emails were removed because of this. These emails were received at the end of 2011 before the observation took place. After looking through the emails, I had to request more emails because there were many emails written to external clients, which I had to discard since the present study focussed only on internal communication. During the period of observation at the workplace in May 2012, I again requested further emails. At the end of the data collection period, four key participants donated more than 100 emails while another donated only 35 emails. A total of 559 emails were collected. From the dataset, 64 percent were first or primary communications on a specific topic. 
Table 3.5 shows a summary of emails received from the three workplaces.

Table 3.5 Composition of emails received from three workplaces

\begin{tabular}{lcccccc}
\hline \multirow{2}{*}{ Emails } & \multicolumn{2}{c}{ Company NZ1 } & \multicolumn{2}{c}{ Company NZ2 } & \multicolumn{2}{c}{ Company M } \\
\cline { 2 - 7 } & No & \% & No & \% & No & \% \\
\hline New messages & 338 & 55 & 388 & 67 & 360 & 64 \\
Chain emails & 273 & 45 & 187 & 33 & 199 & 36 \\
\hline Total & 611 & 100 & 575 & 100 & 559 & 100 \\
\hline
\end{tabular}

In my study, emails were given a code to differentiate the two types of emails collected. A new or first message was labelled with the initial ' $\mathrm{S}$ '. This type of message does not contain a reply and is usually a primary message. Chain emails which are follow-up or response messages were labelled with the initial ' $C$ '. They are usually created by the Reply or Forwarding function generated by the email system, which signals a reply with 'RE' (reply) in front of the subject line, and signals a forwarded email with 'FW' (forwarded) in front of the subject line. The length of a chain email varies. A short chain email can be a two-message exchange while a long chain email may contain as many as eight or more messages. In the present data, although the same message may appear more than once in the dataset of emails donated by all the key participants, only one message was included in the data analysis. A forwarded message which was redelivered to a new recipient with or without any in-text message or only with ' $\mathrm{FYI}$ ' was considered a new email. Thus it was labelled with the initial ' $S$ '.

In categorising a chain email, the position of the email message was indicated by a lower case i.e. $a, b, c$. For example, if a chain email had three follow-up messages, the messages were labelled by a lower case $a, b, c$ and $d$. The first or primary message was labelled $a ; b$ showed that it was a reply to the primary message $a ; c$ indicated that it was a reply to message $b$; and $d$ showed that it was a reply to message $c$. 
The following figure gives a graphic representation of how a chain email was labelled.

\section{Position of message in a chain email}

Chain 1 contains a two-
message exchange

Chain 2 contains a

three-message

exchange
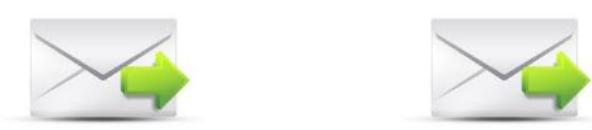

a

b

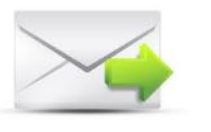

Figure 3.4 Graphic representation of a chain email

The following labelling was employed in the following sequence: initial of type of email; initial of key participant; and a two-digit number such as '01' to show the email number. Thus, message SA01 was a new message number 1 and came from Andy if the email was taken from Company NZ1 dataset. And message CP22b was the second message from chain email number 22 and came from Patrick if the email was taken from Company NZ2. The second letter in the label could belong to either one of the datasets if the key participant had the same initial. In such cases, the workplace where the email was taken is made explicit.

Emails presented in this thesis were used exactly as produced by the key participants, except that to provide confidentiality, pseudonyms were given to the participants and people referred to in the emails, company, locations, dates and other information deemed identifiable to the respective companies.

\subsubsection{Other data sources}

Additional data sources provided me with a range of useful and rich background information. These included consulting the organisation's website, annual reports, internal newsletters and other relevant documents, such as brochures designed for company clients. The organisation's website provided me with valuable information: the structure of the company, the history of the company, the company's portfolio and the different types of services offered. A detailed history of the company was obtained from the CEO of Company NZ1 and 
Company $M$ on the first initial meeting as well as through informal chats with the staff members. For Company NZ2, a brief review of the company was provided by the Divisional Manager and my friend who recommended this company.

\subsubsection{Summary of dataset collected}

Table 3.2 summarises the full dataset from the three organisations - Company NZ1, Company NZ2 and Company M.

Table 3.6 Summary of full dataset

\begin{tabular}{lllll}
\hline Types of data & Details & Company NZ1 & Company NZ2 & Company M \\
\hline Observation Notes & Hours & 150 hours & 82 hours & 50 hours \\
& Duration & 2.5 months & 3 weeks & 1.5 weeks \\
\hline Questionnaire & No. of Respondents & 15 & 21 & 31 \\
\hline Interview & No. of Interviewees & 15 & 17 & 8 \\
& Total Hours & 14 & 6.5 & 4.5 \\
\hline Emails & No. of Participants & 4 & 4 & 5 \\
& Total emails & 611 & 575 & 559 \\
\hline Other data sources & Materials & Annual reports & Annual reports & Internal \\
& & Company's & Internal & newsletter \\
& & webpage & newsletter & Company's \\
& & Brochures & Company's & webpage \\
& & Events' leaflets & webpage & \\
\hline
\end{tabular}

\subsection{Methods of analysis}

Data collected for analysis include field notes, transcribed interviews, questionnaire data, emails donated by key participants and other data sources. Data analysis becomes richer when the findings can be corroborated by comparing the data produced using various methods as a process of triangulation of data (Creswell, 2003). Starfield (2010) also suggests that it is important to triangulate the interpretation of findings with other sources of data collection to address the issue of validity. For this purpose, I employed two methods: first, my interpretations were "cross-checked by [the participants] who supplied the data" (see 'member-checks' Burns, 2010, p. 86); and second, "the perspectives of those relatively uninvolved [in my study were] sought" (see 'peer comparison' Burns, 2010, p. 86). I applied the first method by sending a report written in a form which was comprehensible to non- 
specialists to the CEOs of Company NZ1 and Company M and the Divisional Manager of Company NZ2 once some preliminary analysis had been completed. The report was also made available to all the staff members who participated in this study. To this end, I followed closely the third basic step of the LWP methodology, i.e. provide initial feedback to workplace participants (see Holmes et al., 2011; Holmes \& Stubbe, 2003b; Marra, 2008; Marra \& Holmes, 2008) (see Section 3.3.1). The aim was to obtain feedback or suggestions concerning the content of the report from the participants. I applied my second method by presenting my data at three different meetings at the School of Linguistic and Applied Languages Studies, Victoria University of Wellington: Friday School Seminar, a session of the Research Network for Workplace and Organisational Discourse ${ }^{7}$, and the PhD thesis group. In addition, presentations at conferences also provided useful opportunities to obtain comments from other discourse analysis experts.

The methods employed for data analysis are explained in the following sections.

\subsubsection{Analysing field notes}

Field notes related to the physical layout of the workplace, staff members' workspaces and the behavioural patterns of participants were recorded. These notes were useful in two ways: first, they were useful when interpreting meaning in the email messages, and second, the relevant information was tagged with key words relating to the three crucial dimensions of CofP (Wenger, 1998a) (see Chapter 4). These key words were also used to match email messages which could be interpreted in the light of my field notes. Field notes were labelled FN followed by the date the field note was taken.

\subsubsection{Analysing questionnaire}

The questionnaire data was used as an exploratory device to get the opinion of a wider population and a simple frequency count was undertaken and recorded based on how many participants answered each option in the Likert scale for each item. The frequency count was to obtain percentages which were then used as a basis for identifying trends in the participants' views about email communication.

\footnotetext{
${ }^{7}$ http://lists.vuw.ac.nz/mailman/listinfo/rnwod
} 


\subsubsection{Analysing interviews}

The contents of all recorded interviews were transcribed using the freely available transcription software Transcriber 1.5.1 (see http://trans.sourceforge.net/en/presentation.php) and summarised in meaning units. The transcription provided a rich body of qualitative data for further analysis as described below. Interview transcripts were useful for two reasons: first, they provided relevant quotes from participants; and secondly, they provided relevant information to support the quantitative data collected from the questionnaires. Following the transcription process, I coded the content of the interview transcripts according to themes that were related to the questionnaire and the three criteria defining CofPs using NVivo (NVivo qualitative data analysis software 2010).

\subsubsection{Analysing emails}

The analysis of emails was carried out in two stages. As a preliminary step in analysing the emails, I read the messages through to get a sense of the manner and context in which the email was written. Then I coded the emails according to the communicative functions that they performed and the types of greetings and closing expressions, if used, using qualitative analysis. The systems developed to code emails according to their communicative functions and the types of greeting and closing expressions are described in Chapter 5 and Chapter 6 respectively.

The Statistical Package for the Social Sciences (SPSS 18.0) was used as the statistical tool to analyse two variables (i.e. communicative functions and the use of greetings and closings). The alpha level was conventionally set at .05 for all statistical tests. However, for multiple comparisons, in order to avoid Type I errors, simple Bonferroni correction was used, that is, the significance level was adjusted at a level of .05 divided by the number of comparisons (Larson-Hall, 2010). It should be noted that simple chi-square tests of independence were used selectively throughout the thesis to further support the qualitative analyses, especially in cases where the numbers are particularly interesting in light of other research. 
The second stage involved an in-depth interpretation of the emails. I used Spencer-Oatey's (2000b, 2008) rapport management to frame this work. As stated in Chapter 2, her framework can be used to analyse the management of interpersonal relations beyond the illocutionary domain, to discourse level. In addition, this framework takes into account different workplace cultures. To support my analysis, Halliday's (1994; Halliday \& Matthiessen, 2004) three metafunctions (ideational, interpersonal and textual) were also used to interpret the emails. According to Halliday (2009, pp. 15-16), each of these metafunctions has "its own systems of meaning potential, having as output some specific contribution to the total lexicogrammatical coding". He claims that the three metafunctions are intertwined and do not operate in isolation, and in one grammatical clause, all three metafunctions are being expressed simultaneously. The ideational metafunction is about the meaning of the world, the interpersonal metafunction refers to the meaning of relationships and roles of interactants, and the textual metafunction relates to the meaning of the text. The metafunctions provided additional support to analyse the email messages by investigating the "goings-on"(Halliday, 1985, p. 101), the relationships between the participants and the structure of the email messages. To examine the interpersonal metafunction in written communication, in this case, I focused particularly on the mood choices (i.e. imperatives, declaratives and interrogatives) used in email messages. To explore the ideational metafunction, I analysed transitivity patterns by focusing on process types, particularly the material and mental processes. And to focus on the textual metafunction, I analysed the structure of emails in terms of greetings and closings. By adopting these three approaches, I have been able to provide discourse and pragmatic evidence to show how language is used to maintain social relationship while performing the transactional functions.

In order to demonstrate the application of the three approaches, I include an example analysis and interpretation of a request email from a subordinate, Lesley (HOE) to her superior, Andy (CEO). This email shows how the subordinate manages rapport in the request email to her superior. 
Email 3.1 (CL78a) NZ1

Context: Lesley requests for permission to work from Auckland.

1 From: Lesley

2 Sent: Thursday, 21 April 2011 11:06 a.m.

3 To: Andy

4 Subject: working out of AKL week of 2 May?

5

Hi Andy - [daughter's name]'s going to London on Friday 6th May and I wonder if you would you be ok with me working out of AKL for that week from home as I'd like to spend some time with her in case she doesn't come back for a while and marries Price Harry or something.... I would pay to go up and back myself of course.

As it turns out now, I would also like to meet with [name] and [name] and go through arrangements for AKL coordination and would also have a logistics meeting with [name of client] for the [name of event] event.

Cheers

Lesley

I begin the process of analysis with an overview of the domains through which interpersonal rapport is managed. This analysis supports Spencer-Oatey's (2000a, 2008) and SpencerOatey and Xing's (2003) claim that rapport is managed through a range of domains and that it involves more than the consideration of face sensitivities. Table 3.7 shows an overview of how interpersonal rapport is managed through the five domains. 
Table 3.7 Overview of how interpersonal rapport is managed through the five domains

\begin{tabular}{|c|c|}
\hline Domain & Description \\
\hline $\begin{array}{l}\text { Illocutionary } \\
\text { domain }\end{array}$ & $\begin{array}{l}\text { Strategies used: } \\
\text { Summons: The address line signals that the email is directed to Andy. (line 3) } \\
\text { Announcement: the subject line indicates the topic of the email. (line 4) } \\
\text { Greeting: acknowledging the recipient and expressing solidarity (line 6) } \\
\text { 'Grounder' }{ }^{8} \text { : giving the reason, rationale or justification for her request (line 8). } \\
\text { Humour: injects humour as part of her explanation (line 9). } \\
\text { Head Act: request for permission to work from Auckland (lines } 6 \text { to 7). } \\
\text { Expressing intention: expressing intention to meet with clients while she is in } \\
\text { Auckland (lines } 12 \text { to } 15) \text {. } \\
\text { Closing: expressing solidarity (lines } 17 \text { and 18) }\end{array}$ \\
\hline $\begin{array}{l}\text { Discourse } \\
\text { domain }\end{array}$ & $\begin{array}{l}\text { Lesley has control of the topic but since this is a request to seek permission from } \\
\text { her superior, it is reasonable to expect a reply from Andy. }\end{array}$ \\
\hline $\begin{array}{l}\text { Stylistic } \\
\text { domain }\end{array}$ & Friendly tone and humour is injected into this email. \\
\hline $\begin{array}{l}\text { Participation } \\
\text { domain }\end{array}$ & This email is the first message of a 'conversation' between the two interactants. \\
\hline $\begin{array}{l}\text { Non verbal } \\
\text { domain }\end{array}$ & $\begin{array}{l}\text { No emoticon is present in this email. However, the question mark at the end of } \\
\text { the subject line shows that she is seeking permission. }\end{array}$ \\
\hline
\end{tabular}

Textually, the subject line gives an indication of the topic of the email, written in the form of an ellipsis as in 'working out of AKL week of 2 May?' (line 4). By including a greeting, Lesley is seen to establish rapport with her superior, Andy, by including a greeting ' $\mathrm{Hi}$ ' and acknowledges her by her first name in the email (line 6). In this request email to her superior for permission to work from home, Lesley begins her email by giving an explanation (grounder) that her daughter will be going to London. This is followed immediately by her request for permission to work from her home. When making this request of her superior, she frames it using a few strategies. First, she employs the mental process realised through the use of the verb 'wonder' (line 6); second, the modal 'would' (lines 7, 8 and 10); and third, the conditional 'if' (line 7) are used to mitigate the force of her request. By using the mental

\footnotetext{
${ }^{8}$ Blum-Kulka and Olshtain (1989, p. 205) define grounder as the "reasons for the request" made.
} 
process rather than the material process (as in 'I want to work out of AKL ...'), Lesley is seen as attending to the equity rights of her superior.

She then justifies why she needs to spend the time with her daughter. This justification is accompanied by humour ('... in case she doesn't come back for a while and marries Prince Harry ...' in line 9) which suggests that she is constructing collegiality while making a request of her superior. In an organisation, it is not a common practice to request permission to work from home. Hence this request can be considered a high imposition request. Injecting humour into the request suggests that Lesley attends to Andy's equity rights, i.e. of not being unduly imposed upon, and then she continues to explain that she will pay her own transportation costs (line 10). What is interesting to note here is that she also uses the modality element 'would' and the mental process 'like' (line 12) to signal tentativeness when informing her superior of her intention to meet with some clients, which is work related. It can be interpreted that by using modality and a mental process to realize her intention as she did in line 12, she may be informing Andy that the plans are not confirmed. She could also be protecting herself if the meeting with the clients did not take place.

In this email to her superior, by using some rapport management strategies as well as the lexico-grammatical clauses, Lesley minimizes the threat of her request to her superior's 'quality face' and 'equity rights'. From this example, I have shown how the two approaches (Spencer-Oatey's rapport management and Halliday's metafunctions) can work in tandem to show how language is used to maintain collegiality and solidarity while performing the transactional functions.

\subsection{Conclusion}

This chapter has provided the rationale for using a mixed-methods approach to collect data for my study. The ethnographic perspective as discussed above provided me with tools to understand how the organisation operated, the relationships between the managers and subordinates as well as the work dynamic of the workplace. Applying aspects of linguistic ethnography and the ethnography of communcation enabled me to record communicative events which provided me a deeper understanding of the social practices in the workplaces in order to interpret the language in written text, i.e. email. In addition, the field notes 
collected helped me to form a big picture of how the three workplaces were constituted as communities of practice. I have also described the settings for my study and detailed the methodological steps involved in collecting and analysing the field notes, questionnaires, interviews and emails.

The following four chapters present the analysis and findings. Chapter 4 presents the three workplaces as CofP while the remaining chapters focus on the email data. Chapter 5 discusses the categorisation process of emails into their main communicative functions, and Chapter 6 focuses on the types of greetings and closings in the emails. Chapter 7 presents the results of the analysis of emails focusing particularly on requests. 


\section{Chapter 4: The Workplaces as Communities of Practice}

\subsection{Introduction}

This chapter provides an introduction to the two New Zealand workplaces and one Malaysian workplace. It provides a description of Company NZ1, Company NZ2 and Company $M$, their workplace cultures, their linguistic and non-linguistic behaviours. The workplace culture $^{9}$ of each workplace will be exemplified through a combination of information obtained from observations, questionnaires, interviews, and other documents collected from the workplaces. As discussed in Section 2.4.2, it is the workplace culture that governs the appropriateness and/ or acceptability of how certain things can or cannot be done in a particular way. It is important to note that communication does not exist in a vacuum, and that people's language and behaviour are influenced by their workplace culture. The linguistic choices that people make play an important role in constructing the individual's identity as well as the organisation's identities and culture. In other words, workplace culture is constantly being instantiated in how people do things and on-going interactions (Miller, 2009).

In this chapter, I adopt a Community of Practice (hereafter CofP) (Wenger, 1998a) framework because it allows me to gain insights into the characteristics of workplace culture such as the participants' behaviours, language, values and beliefs and to discuss the different aspects that shape the workplace culture of each company - Company NZ1, Company NZ2 and Company M. Wenger's three criteria (mutual engagement, joint enterprise and shared repertoire) are important in identifying each workplace as a CofP, all of which were evident in the three workplaces. The CofP framework also provides an avenue to explore how staff members 'assert' power and 'do' collegiality.

As explained in Section 3.2, ethnography is a vital tool when using a CofP approach and when examining the interpretation of events in the context in which they occurred. To

\footnotetext{
${ }^{9}$ Workplace culture can be defined as follows "workplace cultures revolve around the shared values and attitudes and the shared experiences that validate them. A culture includes everything that is learned and shared by its members: its social heritage and rules of behaviour, its own customs and traditions, jargon and stories" (Smircich, 1983, cited in Holmes and Marra, 2002: 1684).
} 
understand the workplace culture of each organisation as an outsider, the most appropriate way of initially accessing the culture seemed to be through the lens of what is first presented to the observer, i.e. artefacts ${ }^{10}$, physical layout and workspaces. These aspects of the workplace culture, based on my observations, were supported by the findings from interviews, as well as by conversations with staff members over my period of participant observation in the workplaces.

This chapter is organised as follows. I begin with a discussion of CofP, a theory which has been developed within a social constructionist framework as a valuable concept for analysing an individual's behaviour in a specific working group. Then I discuss what defines each workplace as a CofP. Next, I describe the complexities of the CofPs and take account of how new members in the three workplaces are inducted into the CofPs and how core members differentiate themselves from others. The chapter concludes with a brief discussion comparing all three workplaces.

\subsection{Communities of Practice}

A CofP framework focuses on the discourse which people use to construct their identities in a group, such as in a particular working group, and it has been a useful tool for sociolinguists for analysing interaction at a micro level (Corder \& Meyerhoff, 2007; Eckert \& McConnellGinet, 2003; Marra \& Holmes, 2007; Wenger, 1998a). Given the present research is a comparative study of three different organisations it will involve not only micro analysis but also macro analysis to present similarities and differences between these three workplaces. Therefore the aim of this chapter is to demonstrate that each workplace can be considered a CofP and to compare how the participants in each company construct their membership in the group.

CofP is a social learning theory introduced by Lave and Wenger (1991). This theory was further developed by Wenger (1998a) and has been adopted by sociolinguists in

\footnotetext{
${ }^{10}$ Artifacts refer to "everything from the physical layout, the dress code, the manner in which people address each other, the smell and feel of the place, its emotional intensity, and other phenomena, to the more permanent archival manifestations such as company records, products, statement of philosophy and annual reports" (Schein, 1990, p. 111).
} 
investigating language use in society (Eckert \& McConnell-Ginet, 1992, 1999; Holmes \& Meyerhoff, 1999; King, 2011; Murata, 2011; Schnurr, 2009; Schnurr, Marra, \& Holmes, 2007; Wilson, 2011). Of late, the theory has also been accepted as an organisation tool in business to capitalise on shared knowledge (Fletcher, 2011). The theory distinguishes itself from other approaches by emphasising the concept of mutual engagement and practices ${ }^{11}$. So what is community of practice? According to Wenger and Snyder (2000, p. 139), communities of practice is a term that refers to "groups of people informally bound together by shared expertise and passion for a joint enterprise." In other words, the term community of practice refers to a group of people who are engaged mutually in a common endeavour; and through such mutual engagement, common practices such as "ways of doing things, ways of talking, beliefs, power relations - in short, practices" emerge (Eckert \& McConnell-Ginet, 1992, p. 464). Through sharing experiences and information with each other in a group, group members learn from each other and they have the opportunity to develop themselves both personally and professionally (Lave \& Wenger, 1991). In other words, "practice [which involves the negotiation of meaning] is about meaning as an experience of everyday life" (Wenger, 1998a, p. 52). This in turn involves the confluence of two processes: participation and reification in order for negotiation of meaning to take place (Wenger, 1998a).

In order to be considered a CofP, a group must fulfil at least the following three main criteria:

a. mutual engagement

b. joint enterprise

c. shared repertoire

(Wenger, 1998a)

I exemplify each of these criteria by drawing on examples collected from my pilot study (see Section 3.5.1) which was conducted over a two day period at a university department (pseudonym QS) in December 2010.

Mutual engagement refers to on-going regular interactions. These interactions can take place casually, intensively or comprehensively (Holmes, Stubbe, \& Vine, 1999). Some examples of casual interactions are conversations which take place along the corridor or at

\footnotetext{
${ }^{11}$ See Holmes and Meyerhoff (1999) for a comparison between CofP and other social concepts such as social identity and speech community.
} 
morning tea (Holmes \& Meyerhoff, 1999). The staff in QS interact regularly: casually, they meet at the same time every day for morning tea to 'catch up' with each other or just to 'unwind' and sometimes they interact when they pass each other in the corridor or stairs; they meet intensively as a large group for meetings once a month to discuss work-related issues; or they meet in pairs or small groups for specific discussions. By fulfilling this aspect of CofP, the staff in QS can be considered a potential CofP.

Joint enterprise refers to a "process, not a static agreement" (Wenger, 1998a, p. 82). Wenger (1998a) states that joint enterprise is a negotiated enterprise, and is not just a stated shared goal. Joint enterprise involves the complex relationships of mutual accountability that become part of the practice of the community. For example, the stated goal of the QS group was reflected in their shared institutional objective. The staff members work together towards the achievement of this shared institutional objective which is to provide excellent customer service to students who enrol in the university. According to Wenger's (1998a) theory, the staff members in QS constituted a CofP because they were engaged in an on-going process of negotiation in their attempt to contribute towards the larger enterprise. This negotiated enterprise or shared goal reflected the staff members' own understanding of their respective roles within the department. It is the fulfilment of this characteristic that defines the staff members in QS as a CofP.

As time progresses, the joint enterprise will develop a repertoire of resources shared for the purpose of negotiating meaning (Wenger, 1998a). In other words, shared repertoire refers to a common set of linguistic resources which is understood among group members (Wenger, 1998a). According to Holmes and Meyerhoff (1999), social norms and behavioural expectations form part of a workplace's shared repertoire and this contributes to the formation of a CofP. Some examples that may contribute to a shared repertoire are linguistic routines, gestures and regular joint meals (Holmes \& Meyerhoff, 1999, p. 176). In QS, for example, I observed regular greeting rituals such as 'Hello', 'Good morning' and 'Cheers' exchanged between members. Another example is the members at QS seemed to be able to treat conversations as though they were continuing the discussion where they had previously left off. This feature of lack of preambles provides the basis of identifying the staff in QS as a CofP (Wenger, 1998a). 
As a summary of the discussion above, through members' mutual engagement in a joint enterprise, the discursive behaviours of members in a community may develop which eventually results in the development of a shared linguistic repertoire and agreed ways of communication. This shared linguistic repertoire may be reflected in specific linguistic strategies members draw on and precise interaction patterns that are displayed in daily interactions. By applying these three criteria to the workplace, a particular team or working group and the organisation as a whole can be considered a CofP (Wenger, 1998a, p. 125).

How does the researcher determine that the group of people being examined constitute a CofP? What yardstick is used to verify that the three criteria of CofP are present? To answer these questions, Wenger (1998a, pp. 125 - 126) outlines a list of 14 specific features through which the main three criteria (i.e. mutual engagement, joint enterprise and shared repertoire) discussed above are instantiated. These features include both linguistic and nonlinguistic aspects which form the basis of workplace cultures, but the following nine are particularly relevant in the present research:

- sustained mutual relationships - harmonious or conflictual

- shared ways of engaging in doing things together

- absence of introductory preambles, as if conversations and interactions were merely the continuation of an on-going process

- knowing what others know, what they do, and how they can contribute to an enterprise

- mutually defining identities

- specific tools, representations, and other artifacts

- local lore, shared stories, inside jokes, knowing laughter

- jargon and shortcuts to communication as well as the ease of producing new ones

- certain styles recognized as displaying membership

Holmes and Meyerhoff (1999) comment that the above features can be used as a means of identifying a CofP's distinct qualities. More specifically, these features can be used as a tool to ascertain whether a group constitutes a CofP at all and to compare the group's communicative practices. Some examples of workplace shared repertoire taken from the Victoria University of Wellington LWP (see Section 3.4.1) are regular greeting rituals, the 
exchange of small talk, how directives (Vine, 2001) are given and how humour functions in workplace meetings (Holmes \& Marra, 2002; Marra \& Holmes, 2007).

As stated above, the three criteria discussed in the previous sections are considered the main principles of a CofP approach. In addition to the three criteria, the element of identity (similar to that of the social identity theory (Holmes \& Meyerhoff, 1999)) is also important in a CofP approach. The element of identity presents individual identity and group identity as being dependent on each other. One aspect which differentiates Tajfel's Social Identity theory (see Holmes \& Meyerhoff, 1999) and CofP is that the latter also builds in a goal (i.e. joint enterprise). In other words, the identity that members bring to the group helps shape the identity of the group and it is this identity which is jointly constructed. It is this notion of group identity which makes the CofP approach useful for this research, as one of the goals of this research is to determine how linguistic and non-linguistic behaviours and practices construct an identity for the staff members in their respective workplaces.

There are several advantages of using a CofP approach in this thesis. One of the advantages is the criterion of shared construction and the ability to re-negotiate practices. It is perhaps this criterion which makes the CofP approach most useful for this research, as staff members in the three workplaces were seen to continually re-negotiate their shared practices. One example which shows the advantage of using a CofP approach to discover the shared practices within the CofPs identified at a school is Eckert's (2000) seminal study of a group of students at Belten High School. Her study, which combines an ethnographic approach and linguistic analysis of shared practices of the students at this school, provides an insight into the co-constructed shared practices of a wider community, which in turn determines the community identity as a whole. While an individual may choose to join a social group in a high school (Eckert, 2000) through mutual engagement, they are only accepted as a member once they have conformed to the social behaviour of the group that they joined. Having said this, people need not develop shared practices so that they are considered communities of practice because there exists a variety of 'modes of belonging'. Wenger (1998a) lists three modes of belonging: engagement, imagination and alignment. Members need to be aware that there must be active involvement in their engagement in the community to prevent marginalisation (Wenger, 1998a). Through communities of imagination, members imagine themselves to have something in common with other individuals, for example, sharing the 
same trait or belonging to the same level of hierarchy in a workplace context. Likewise, through communities of alignment, members can 'align' themselves as part of an organisation, but that alignment is considered compliance rather than engagement.

Wenger (1998b, p. 3) claims that membership in an organisation is based on "participation rather than an official status" and an individual joins a CofP as a peripheral member. Once new members have acquainted themselves with the ways of doing things and talking, familiarized themselves with and assimilated the beliefs held by the CofP, and understood and observed the power relations that exist in the CofP, the new members may be accepted as core members. In other words, the core membership requirement of a CofP is satisfied when members acquire a 'certain level of sociolinguistic competence' (Holmes \& Meyerhoff, 1999, p. 174). However, it is important to note that the degree of membership and the social status of members within the CofP may vary (Wenger, 1998a). This is because the identities and status of members are constantly in the process of being modified, negotiated and reified.

The CofP approach allows for a member's alignment to change over time. This can be explained using the concept of trajectory. A member may be labelled as peripheral but on an inbound trajectory; this type of member does not exhibit all the core practices which form part of the CofP's repertoire but over time, may be accepted as a core member when these practices are adopted. The approach also allows for a hierarchy to exist within a CofP and this enables the higher status member to have more influence over what is accepted as shared practice (Eckert, 2000; Eckert \& Wenger, 2005). Davies (2005) comments on this point and suggests further investigation of the relationship between power and hierarchy in legitimising membership in a CofP. This situation is common in workplaces where the superiors tend to have greater influence in what is accepted as shared practice, as shown later in this chapter.

A CofP develops with "an aggregate of people who come together around mutual engagement in an endeavour" (Eckert \& McConnell-Ginet, 1992, p. 464). In the case of workplaces, this endeavour is to perform the transactional duties which the members are paid to do. CofPs found in workplaces are on-going entities and could potentially last for a long time unlike, for example, the CofPs in rugby teams as Wilson (2011) demonstrated in 
his research. New members who join a workplace CofP will learn the communicative and social practices in order to align with the group and even though these practices may change, the CofP may remain the same because there is a continuation of negotiated practice.

As there are many work groups in a workplace, Wenger (1998a) identifies a diverse community which encapsulates a broader scope of engagement than a single CofP as a Constellation of Practices. He notes that such a configuration may be "a large configuration such as the global economy, a city or a social movement, or a smaller configuration such as a factory, an office or a school" (Wenger, 1998a, p. 127). This concept has been further developed by other researchers (Brannan, 2007; Rock, 2005). For example, Brannan (2007) uses this concept to describe how sexuality is constructed in a call centre; and Rock (2005) uses it to examine the discursive practices that a police force used in interviews with detainees during police interviews.

According to Wenger (1998a), viewing an organisation as a constellation fulfils two functions: it is a way of recognizing it as a community, and it takes into account important discontinuities between individual communities. Wenger (1998a, p. 127) suggests the following criteria as a guideline for considering an organisation as a constellation of practices:

1. sharing historical roots

2. having related enterprises

3. serving a cause or belonging to an institution

4. facing similar conditions

5. having members in common

6. sharing artifacts

7. having geographical relations of proximity or interaction

8. having overlapping styles or discourses

9. competing for the same resources

Based on the guideline, all three organisations in my research can be viewed as constellations, as I will demonstrate in this chapter. 
As practice does not exist in a vacuum, Wenger (1998a) recognizes that physical distance between members in a CofP can be a factor that cause CofP to fizzle out or fail. He argues that CofPs are shaped by the active participation of participants rather than being defined by a physical form.

My argument is not that physical proximity, institutional affiliation, or frequency of interaction are irrelevant, but rather that the geography of practice cannot be reduced to them. Practice is always located in time and space because it always exists in specific communities and arises out of mutual engagement, which is largely dependent on specific places and times. Yet the relations that constitute practice are primarily defined by learning. As a result, the landscape of practice is an emergent structure in which learning constantly creates localities that reconfigure the geography. (Wenger, 1998a, pp. 130 - 131)

Wenger, McDermott and Snyder (2002, p. 115) discuss the challenges of geographical separation in what they label as "distributed communities" (referring to communities that cannot rely on face-to-face interactions as their main channel of communication). They attribute the issues of space and place as a problem because physical proximity facilitates connections between members, including those who are marginal. Although they argue that "members located in the same building or town often see each other by chance - in the hall, in the elevator, at meetings, or in the lunchroom", they emphasise that "distance simply makes it more difficult" (Wenger et al., 2002, p. 116). In other words, the use of space and the relative distance between staff members can potentially have an influence on the communicative norms that define them. As shown by Wilson (2011) in his study of rugby players, the Forwards had more opportunities to participate in phatic communication and do solidarity than the Backs due to the relative distance between the players. Similarly, in the workplace, physical location and workspaces also have the potential to influence the discourse strategies that staff members employed.

\subsection{Company NZ1 as a community of practice}

As introduced in Section 3.3.1, Company NZ1 was a non-profit organisation and its core business was oriented to events management. This company had a change of name in 2009 and went through a restructure when a new CEO was appointed. The new structure which was made up of three groups - Corporate Team, HOE and Support Team - is an example of a 
constellation. Although all the staff members in each team had their own specific focus and job responsibilities, each of these communities contributed to the overall constellation.

So what makes Company NZ1 a CofP? The discussion that follows will focus on the three criteria: joint enterprise, mutual engagement and shared repertoire.

\subsubsection{Joint enterprise}

Company NZ1's goal was not just a stated shared goal but a negotiated enterprise which involved "mutual accountability" that was one component of the CofP (Wenger, 1998a, p. 81). The staff members of Company NZ1 were very committed to their clients and they aimed to provide them with quality events and excellent customer service. To illustrate this point, I make reference to an example. A technical glitch in the online registration of one event resulted in a series of clients' complaints and this problem would eventually have serious implications for the company. To solve this problem, clients were requested to register manually although this meant additional administrative work for the staff members. I noted that staff members in Company NZ1 paid attention to customers' needs when solving this problem became their priority (FN 17 March 2011). I wrote in my field notes "Due to a technical glitch, all registrations for [name of event] had to be manually keyed into the clients' database. As this job was tedious and time consuming, all staff members were involved. Everyone, including myself offered to help and we were each given a stack of registration forms to be manually keyed into the database. At about $11 \mathrm{am}$, Andy, the CEO, organised an impromptu meeting and Lesley, the HOE, and Rose, the support team member, were called into her room. I was later informed by Rose about the purpose of the meeting to find a solution for the online registration problem." The team effort described above shows that everyone believed that customers' satisfaction was important. In other words, keeping customers happy was their collectively negotiated enterprise. Unfortunately, one week later, the problem was still unresolved by the IT provider. Lesley was quick to address the problem and sent an email to Andy "sorry to escalate this but is [it] seems that the registration is still not fixed." This email further shows the staff members' commitment to keeping their customers happy, in this case, the ability to register without hassle. Thus, it is evident that providing excellent customer service to clients was a joint negotiated enterprise. 


\subsubsection{Mutual engagement}

Mutual engagement takes place anywhere from work-related, formal discussions to casual, informal interactions, as people pass each other in the corridor or "share morning tea" (Holmes \& Meyerhoff, 1999). The availability of the physical space and the open layout of the office in Company NZ1 facilitated interaction and promoted members' participation, making mutual engagement possible. At the centre of the office was a room occupied by four support team members with their desks located at each corner of the room (see figure 4.1). This room which was converted from a board room, previously used for meetings, was a busy spot in the office. Staff members usually congregated at a round table in the room for discussions of both work and non-work related issues. The door to this room was always open and this allowed other staff to walk in and out of the room freely. A full wall of this room had been re-constructed to a half wall which not only allowed light into the room but also created the impression of an organisation that emphasised open communication. This layout appeared to facilitate free flowing conversations: on many occasions I observed staff members call out to each other and pass documents to one another over the half wall without having to enter the centre room. There were also many instances of exchange of social talk as staff members stood at the half wall. In addition, the open-plan work area in the centre room also facilitated interaction as conversations often broke out between the four support members while they were sitting at their desks. In other words, the space and relative distance between the staff members gave them the advantage of doing solidarity and maintain rapport as shown later in the chapter. This observation mirrors that of Wilson's (2011) study of rugby players where the relative distance between the Forwards gave them greater advantage to build rapport and participate in social interaction.

Staff members met on a regular basis, fulfilling the criterion of "mutual engagement" (Wenger, 1998a, p. 73). Meetings provided an avenue for staff members to update each other on work related issues, achieve workplace goals, organise and plan work schedules, solve problems and make decisions. Meetings were crucial to negotiate and align business goals and objectives. During these weekly meetings, all staff members presented a report on their work and discussed issues related to the events the company conducted (joint enterprise). It was also during these meetings that they exhibited an understanding of each other's roles (shared repertoire). These meetings were also evidence of the expression of 
"legitimate power"(French \& Raven, 1968, p. 264) in that the CEO, Andy, was in control of meetings in that she decided what went on the agenda and chaired these meetings. According to Holmes, Marra and Vine (2011, p. 61), the chair "represents the voice of conceptualised authority". In other words, setting the agenda of the meeting and chairing these meetings clearly demonstrated the power of the CEO. From the interviews conducted, I understood that these weekly meetings also provided an opportunity for staff members to plan, solve issues, and to improve rapport and relationship with staff members. In one meeting, staff members were asked to consider their roles and job descriptions after the restructuring process. As Wenger (1998:74) states, unless the members in a CofP are "included in what matters" in the community, a CofP is not likely to coalesce. By getting the staff members involved in the restructuring process, i.e. soliciting their opinion, Andy ensured that all the staff members played a core role in the development of the CofP. While meetings fulfil the criteria of mutual engagement, they are also one way of 'doing power' in workplaces. One of the most obvious ways in which one 'does power' through meetings is by determining what goes on the agenda, as illustrated in the discussion above.

Other activities in which staff members "engage" with each other in regular interaction are celebrations, social gatherings and small talk. Small talk, the shared repertoire observed in this workplace, was common at the beginning of the day. For example, when I arrived at the office one morning, Jean and Pat were engaged in small talk with Rose and Teresa in the centre room (FN 30 March 2011). Small talk also took place before requests were made. I wrote in my field note on 15 March 2011, "Jean asked Joline about her son's health before giving instructions on a work related task". Such exchanges typically softened the transition to work related instructions and attended to the face needs of the hearer (Holmes \& Stubbe, 2003b).

My observation over a period of about two and a half months suggested that the boundary between work talk and social talk was relatively weak in the office. Conversations broke out throughout the day. Small talk covered a wide range of standard topics such as the weather, fashion, mention of family and health. Some specific examples of small talk topics at Company NZ1 were the aftermath of an earthquake in Japan, travelling to the US and diet. Humour was also injected into these conversations. This could be seen when the support 
team members chatted with each other between intervals of work. These strategies are ways of reinforcing group solidarity and 'doing collegiality'.

\subsubsection{Shared repertoire}

Shared repertoire refers to "joint resources for negotiating meaning" (Holmes \& Meyerhoff, 1999, p. 176), including "routines, words, tools, ways of doing things, stories, gestures, symbols, genres, actions, or concepts that the community produced or adopted" (Wenger, 1998a, p. 83). This also includes "specialised terminology and linguistic routines" which Holmes and Meyerhoff (1999, p. 176) view as "fruitful" for discourse analysts.

At Company NZ1, shared repertoire is observable in regular greeting rituals face to face, greetings and closings in email messages, celebrations, the absence of preambles, the use of jargon, and the way of getting things done.

Staff members used an informal mode of address and first names were the preferred choice. They greeted each other at the first encounter of the day with 'Hi' or 'Good morning' and in passing. When staff members received external phone calls, the standard greeting was 'Good morning/ afternoon + [name of person who answers the call] speaking'. Sometimes the widely known Maori greeting 'Kia Ora' was used. Similarly, there was no specified way of writing an email. Greetings used in email communication were informal with the most common form being 'Hi + first name'. Like greetings, an informal form "cheers" was used to sign off an email. Some staff members signed off with their first name and some with only their initials. See Section 6.3 for a detailed analysis of the use of greetings and closings in emails.

Celebrating staff members' birthdays and special occasions was a common practice in Company NZ1, one aspect of their "shared repertoire" (Wenger, 1998a, p. 82) of practices and has become part of the company's practice. Unlike staff meetings which enacted power from the top, celebrations of staff members' birthdays were initiated by the staff members themselves. These celebrations are examples of manifestations of collegiality and solidarity, i.e. aspects of rapport management (Spencer-Oatey, 2000c). Although staff members did not have morning tea together every day, they usually celebrated these occasions during 
morning tea. The person who celebrated his/ her birthday usually brought a cake to share with their colleagues during morning tea. These invitations were announced verbally on the day itself. For example, Angela gave a morning tea shout ${ }^{12}$ to celebrate her birthday (FN 30 March 2011). I wrote in my field notes "Angela rang the bell at the reception area and announced 'Morning tea' twice. Everyone made their way to the meeting room where the celebration took place and a variety of cakes, biscuits, muffins, homemade pies and coffee and tea were served".

Besides birthday celebrations, having lunch together was another common practice. Usually the same group of staff members gathered together in the meeting room for lunch. In addition to the centre room as an ideal place for mutual engagement, the meeting room performed the same function. In fact it had a dual function - transactional and interpersonal. Staff members used this room for their weekly meetings and social gatherings, i.e. lunch and birthday celebrations. The fact that this room was available for staff members to use to have lunch facilitated social interaction and staff members had the opportunity to 'do solidarity' and 'do collegiality'. It also gave them a sense of belonging together and prevented them from being marginalised. I wrote in my field notes "staff members use the meeting room for morning tea when there are birthday celebrations. This room is also very popular during lunch time as staff members gather together for lunch. There is lots of laughter, jocular abuse and social talk" (FN 12 April 2011).

During lunch time, they engaged in conversations of various topics and there was lots of laughter and teasing humour, an expression of solidarity (Hay, 2001). For example, on one occasion (FN 8 March 2011), while having lunch together, some of the staff members teased Angela who was addicted to Diet Coke and joked about her excessive intake and the impact it would have on her health. Angela did not take offence at that comment but contributed to the joke, as "[a] shared way[s] of engaging in doing things together" (Wenger, 1998a, p. 125), by making reference to how much money she could have saved if she stopped her addiction. This example of supportive humour demonstrates an important aspect in workplace

\footnotetext{
${ }^{12}$ The word 'shout' is a popular colloquial term used in New Zealand and was first associated with the country's beer drinking culture. According to Bennett (1943, p. 89), 'to shout' means " to 'stand a drink', to shout an order, at the bar; but it now also means to treat another person, no matter what the place or occasion". Similarly, Bardsley (2013, p. 343) defines 'shout' as 'to buy a round of drinks'.
} 
interaction and provides a basis for this workplace's distinct culture (Holmes, 2000a; Holmes \& Marra, 2002; Holmes \& Stubbe, 2003b; Marra \& Holmes, 2007).

As stated in Section 4.2, another possible feature of a CofP is the absence of introductory preambles. Staff members seemed to be able to pick up a conversation after a short or long break and continue from where they had left off. One example is when Rose asked Annette about their mutual friend's latest update (FN 9 March 2011). While I was unable to follow the conversation, Annette seemed to know who Rose referred to although the name of their mutual friend was not mentioned.

In addition to the areas discussed above, Company NZ1 had its own in-house jargon, mostly referring to specific events that the company ran. For confidentiality reasons, I cannot present specific examples. A newcomer needed to become familiar with this shared language in order to fit into the CofP (Wenger 1998). Familiarity with the names of the events was also essential to perform one's job efficiently. The main point here is that these locally negotiated terms and specialised jargon are not part of a newcomer's linguistic repertoire and John, a newcomer to the staff, was unable to participate actively in the discussions with the members of the Support Team in his first two weeks. His failure to participate actively was an indication of his lack of access to the shared repertoire which the community had already developed (i.e. the jargon used to refer to the names of events). This example provided evidence that the ability to understand and take an active part in team discussions (localised practice) show that core members were separated from those who were either peripheral or marginal members (Wenger, 1998a, p. 166).

Getting things done at work is "the result of a collective process of negotiation that reflects the full complexity of mutual engagement" (Wenger, 1998a, p. 77). Different workplaces develop distinctive discursive practices in the way they get things done in the office. Some practices adopted convey power while others do solidarity. Language is one way by which meaning is reified and these shared practices are jointly negotiated. In workplaces, staff members use a range of communicative functions to get work done. See Section 5.3.1 for the discussion of the main communicative functions in Company NZ1. Making requests is one typical example of how people get things done in a workplace environment and they are done either face to face or via email. 
In Company NZ1, requests were made face to face and via email. Verbal requests were made in a casual manner. When requests were made face to face, small talk usually occurred in between the conversation or at the beginning of the conversation. Small talk "expresses and reinforces solidarity; and it is a way of doing collegiality" (Holmes \& Stubbe, 2003b, p. 107). Two examples are given to support this observation. The first example was taken from my observation field notes on 7 March 2011 when Symone came to Joline's desk and sat next to her while they composed an email together. Symone wanted the email to be sent out to the company's clients. Rather than dictating to Joline how the email should be written, drafting the email together shows that Symone encouraged an active discussion of exploring the possible words and phrases to be used in the email. Another example was noted on 22 March 2011 when Jean came to Annette's desk to give her a task to do. Rather than sending the request via email or logging it in the internal task request system ${ }^{13}$, Jean chose to do it face to face. All requests were made in a casual and polite manner and always ended with 'thanks'. When requests were made via email, humour was used as one strategy to soften the illocutionary force of the requests. See Sections 7.5.1 and 7.6.1 for discussion of how requests were made via email. This discussion shows that although the tasks were predominantly transactional in function, the different ways of conveying the requests clearly suggest that attention was paid to the interpersonal aspects of interaction, i.e. rapport management (Spencer-Oatey, 2000c).

Another feature of the shared repertoire observed at Company NZ1 was that emails sent in response to a request were typically sent without any messages in the body of the email. These emails were usually a follow up from verbal discussions. This shared repertoire is one means of instantiating one of Wenger's (1998a, p. 126) features of CofP, namely "certain styles recognized as displaying membership". Staff members who were interviewed said that it is not necessary to include a note in the body of the email because 'it is understood'.

The shared discourse repertoire observed in this workplace provided evidence of how the participants cement solidary relationships. By participating in these shared repertoires which

\footnotetext{
${ }^{13}$ The internal Task Request System has been used to modify the use of email in Company NZ1. This system was created to log internal requests/jobs to be carried out by staff members. When someone has a task for another staff member, the task request system is used as a replacement for the former use of email. This system was created (1) to reduce the number of internal emails and (2) to monitor tasks that need to be carried out within a certain time frame.
} 
comprised "routines, words, tools, ways of doing things, stories, gestures, symbols, genres, actions or concepts" (Wenger, 1998a, p. 83), the staff members developed an identity which created a sense of belonging to a CofP. These shared repertoires helped to create bonding and construct solidarity among the staff members. In other words, when staff members were able to contribute actively in formal or casual conversations, they were able to contribute positively to the dynamic interaction. Sharing ways of engaging in doing things together through the use of supportive humour also demonstrates the staff members' construction of membership in the workplace. In addition, the open layout of the office provided opportunity for work colleagues to 'do collegiality' and acquire a sense of collective identity, an observation which concurs with that of other researchers (Jewson, 2007; Wenger et al., 2002; Wilson, 2011).

In summary, Company NZ1 fulfilled the three criteria of a CofP as listed below:

- Jointly negotiated enterprise - their goal was to provide quality events and excellent customer service for the company's clients;

- Mutual engagement - staff members' regular interactions at weekly meetings, birthday celebrations and during lunch hour; and

- Shared repertoire - they shared common linguistic and non-linguistic practices such as in-house jargon which referred to names of events, informal greetings and closings used in emails, informal verbal greeting rituals and the exchange of small talk and collaborative humour between staff members. 


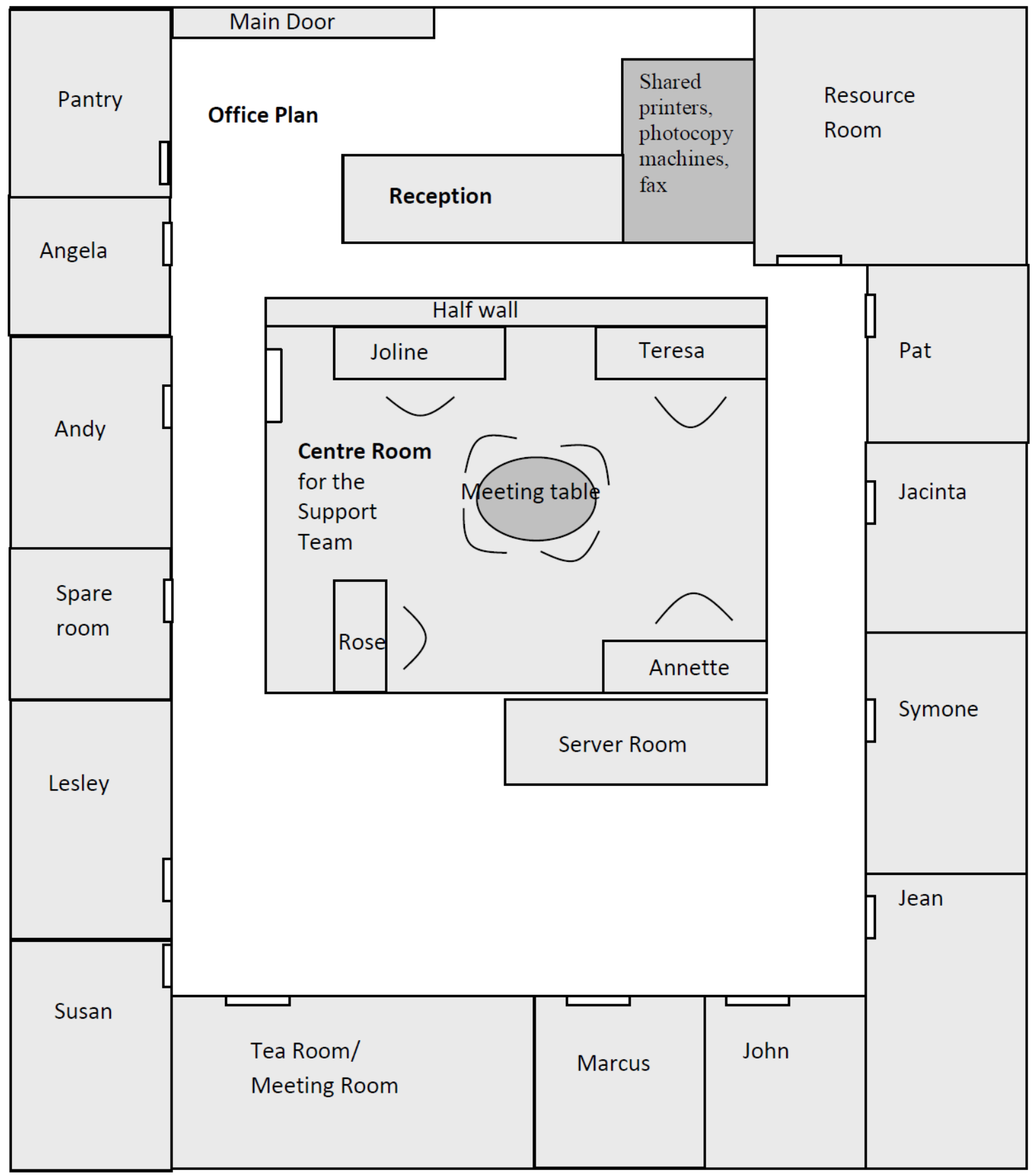

Legend:

Door -

Figure 4.1 Layout of office (Company NZ1) 


\subsection{Company NZ2 as a community of practice}

Company NZ2 was a large commercial organisation whose main focus was construction. It was part of a large international organisation with its main headquarters in Australia. The New Zealand division began operation in the 1800s and provided employment for almost five thousand people. A large company like Company NZ2 is an example of a Constellation of Practice, i.e. this organisation was made up of multiple embedded CofPs. The various departments (e.g. administration, finance, upper management, engineering, and other cross functional departments) and work groups (e.g. white collar professionals and blue collar construction workers) in Company NZ2 all contributed to the overall organization even though each department had its specific goal and practices. This chapter, however, deals primarily with the white collar professionals because the blue collar construction workers were not involved in email communication, which is the focus of this study.

So what makes Company NZ2 a community of practice? The discussion that follows focuses on the three criteria: joint enterprise of a construction company, mutual engagement and shared repertoire.

\subsubsection{Joint enterprise}

Staff members in Company NZ2 came together around the joint enterprise of their shared goals as a construction company (Wenger, 1998a). Their common objective, i.e. providing customer satisfaction in relation to delivery of required construction, was evident from the company's Quality Policy which stated 'meet the needs of its customers by providing products and services to the standard of quality and delivery expected by them'. Employees were made aware of the Quality Policy as it was displayed on the walls in the various offices, reception area, lunch room and laboratory. Although one may argue that it was an edict from top management, there was also evidence that employees shared this same objective. I refer to the following example extracted from my field notes. In one meeting which I had the privilege to attend, Patrick reminded his subordinates to be courteous and to behave professionally at all times (FN 30 August 2011). One subordinate replied in response to Patrick's comment "we always put customers first". This example shows that the employees also contributed to the joint enterprise. 
In another example, on 15 August 2011, Wellington was hit by a snow storm. Patrick and his team were involved in clearing the roads in the city from snow and had been working hard the entire night to ensure that customers' complaints were addressed in an efficient and timely manner, in line with the company's Quality Policy. Sheila sent an email which read 'Can you please check and provide contact details of those that may be available to be called on tonight and what vehicles and/ or licences they hold.' to all staff members on 16 August requesting volunteers as a back-up crew for Patrick's team. Several departments responded with a list of volunteers. While this example clearly shows Company NZ2's team spirit, it also exemplifies a shared negotiated enterprise. In sum, the two examples described above indicate that staff members do come together as a joint enterprise.

\subsubsection{Mutual engagement}

Mutual engagement involves regular interaction and the availability of physical spaces make this possible (Wenger et al., 2002; Wilson, 2011). As discussed in Section 4.3.1, physical spaces and an open layout typically facilitate and encourage interaction in Company NZ1, however, quite the opposite was observed in Company NZ2. Company NZ2 had a large construction depot with a main administration office building, a yard, truck storage, a sales office and a laboratory. The sales office and laboratory were located about 200 metres away from the main administration office building which had two levels: the first level was occupied by the management team and the ground level was occupied by one department which had three sub-divisions - engineers, supervisors and the administrative staff. While staff members of the management team had their own rooms on the first floor (see Figure 4.2), staff members occupying the ground floor observed an open office plan (see Figure 4.3). Generally the office on the first floor was quiet although occasionally there were some quick exchanges of greetings at the first encounters of the day. From my observation, staff members did not gather around for small talk during office hours. The quietness on the first floor was in contrast with the bustling chatter of the blue collar workers when they gathered for an early morning briefing and afternoon debriefing on the ground floor. While the open layout area in Company NZ1 facilitated and encouraged social interaction between the staff members, the design of the physical space in Company NZ2 did not facilitate interaction 
between the white collar professionals. This clearly challenges the observation of other researchers (Wenger et al., 2002; Wilson, 2011) that space and physical proximity facilitated interactions.

Meeting regularly fulfils the "mutual engagement" criterion which is critical to the development of a CofP (Wenger, 1998a, p. 73). Fortnightly meetings, referred to as Toolbox meetings, were conducted with blue collar workers and I had the privilege to attend one of these meetings (FN 30 August 2011). These meetings which usually lasted for an hour started as early as seven in the morning and were deemed important by both the management and the staff members. According to one site worker, these meetings act as a bridge between them and higher management. He added that the meetings are a good avenue for the blue collar workers to raise issues with the management and for the management to inform the staff about the changes or progress of the company, fulfilling the criteria of a community of practice, namely "joint negotiated enterprise" (Wenger, 1998a, p. 78). During these meetings, managers were required to show the company's monthly DVD which gave an account of the company's development. Meetings conducted were predominantly to fulfil the requirements from top management and clearly constituted an enactment of power and control. This supports Mumby's $(1988$, p. 68) assertion that meetings "function as one of the most important and visible sites of organisational power, and the reification of organisational hierarchy". In other words, meetings are an indication of power from the top management.

I observed that this workplace was predominantly work oriented. Unlike Company NZ1 where staff members usually engaged in small talk before a request was made, in this workplace, requests were made immediately and without preamble; small talk rarely occurred. Sometimes only formulaic exchanges of greetings preceded the requests made. Clearly in this workplace, staff members did not feel obligated to engage in small talk in work transactions. If staff members had the opportunity to gather together during morning tea or lunch, mainly work related issues were discussed during my periods of observation. In my field notes dated 23 August 2011, I wrote "It's about 12 noon, I was invited to join the divisional managers for lunch in the meeting room. In the room there were the Operations Manager, three divisional managers, a consultant from the headquarters in Auckland, a civil 
engineer, Megan, the Senior Administrator and Ken, the Assistant Accountant. The first nonwork related conversation was initiated by the civil engineer who asked the consultant about his trip to the office. After a brief exchange, this conversation immediately switched to workrelated issues". In short, topics related to work dominated the lunch discussion. This example typified the other observations made throughout the period I conducted my field work in this workplace.

\subsubsection{Shared repertoire}

The staff members in Company NZ2 displayed a number of characteristics of shared repertoire. These characteristics formed a basis of distinguishing a unique workplace culture in Company NZ2. Shared practices such as greeting rituals, celebrations, the use of jargon, preferred ways of making requests, the exchange of small talk and how humour is tolerated in the workplace instantiate some of the features of a CofP identified by Wenger (1998a).

As in Company NZ1, staff members greeted each other by first names. Although staff members' positions in the company were determined by the organisation hierarchy (see Section 3.3.1), staff members in higher positions appeared not to consider formality as of high importance. Other address terms 'mate', 'bro', 'guys' ${ }^{14}$ and 'cuz' ${ }^{15}$ were also used to address one another in this workplace. These terms enhance the solidarity of the relationship between the hearer and the speaker (Wilson, 2010). It is interesting to note that the terms 'bro' and 'cuz' were used to non-relations, possibly to indicate the importance of kinship of Maori language address terms (Johnston \& Robertson, 1993, p. 125).This informal term of address was also present in the emails with the most preferred form being ' $\mathrm{Hi}+$ first name'. Familiarizers such as 'guys', 'mate' and 'cuz' were also evident in the emails collected. See Section 6.4 for more detailed analysis of the use of greetings and closings in emails.

When external phone calls were received, staff members always introduced the company's name first. The following are some examples 'Welcome to [company name], you are

\footnotetext{
${ }^{14}$ Leech (1999, p. 110) categorises these terms 'mate', 'guys' and 'bro' as "familiarizers" and they are used predominantly between men. 'Mate' is a popular term in New Zealand as shown in Wilson's (2010) research of address terms used by rugby players.

${ }^{15}$ The term 'cuz' is associated with Maori English and it is referred to as Maori Vernacular English (MVE)(Bell, 2000, pp. 222 -223) and as 'bro talk' in colloquial terms (King, 1999, p. 20).
} 
speaking with [name of person who answers the call]', 'Welcome to [company name + location]' and 'Welcome to [company name], good morning/ afternoon'. Although there was no written policy on the greeting used when receiving external phone calls, the examples above gave the impression that introducing the company's name signals a sense of belonging.

As in Company NZ1, celebrating staff members' birthdays and other events in people's lives was a common practice in Company NZ2, fulfilling the criterion of "shared repertoire" (Wenger, 1998a, p. 82). These celebrations are examples of reinforcing interpersonal relationships between staff members who work together. Like Company NZ1, it was the employee who celebrated his/ her birthday who gave a morning tea shout to all the employees. The morning tea shout usually consisted of a wide spread of choices - meat pies, cookies, cakes with sometimes home baked cookies were also offered. In other words, it was the individual who took responsibility for contributing to the rapport and solidarity in the workplace. During my ethnography period, I observed two such occasions. Sheila, who was celebrating her birthday, treated the staff members to home-baked chocolate cookies in addition to some meat pies that she had bought (FN 22 August 2011). On another occasion, when the Operations Manager celebrated his $50^{\text {th }}$ birthday (FN 7 September 2011), he gave a sumptuous morning tea to the delight of all the employees. It was a common practice for staff members to send out a generic invitation for such occasions on the day itself. Unlike Company NZ1 where invitations for morning tea were given verbally on the day itself, in Company NZ2, emails with the subject heading 'Morning tea shout' were sent to make the announcement. In this case the use of email made the communication easy since it was a big group and staff members were scattered around the construction depot.

These birthday celebrations were held at a sizeable lunch room, also referred to as the 'smoko'. While the meeting room in Company NZ1 provided a space which facilitated and promoted social interactions on non-work related issues between the staff members, the availability of the 'smoko' in Company NZ2 worked differently, i.e. work colleagues discussed only work related matters. In other words, physical space and proximity did little to promote social interactions on non-work related issues in Company NZ2. In addition, staff members interviewed reported that they do not have lunch with their work mates because everyone is 
always on the move - attending meetings and off site work. One staff member commented that she misses her previous workplace where she and her work colleagues always have lunch together. She stated that everyone has lunch alone in this workplace.

Besides birthday celebrations, company events such the staff children's annual Christmas party and various sport events were a common practice in Company NZ2. On one occasion, the staff were treated to a match during the Rugby World Cup 2011. These company events and activities formed an important part of the workplace culture, a negotiated shared repertoire, besides making mutual engagement possible. Clearly these company events provide an opportunity for the manifestation of solidarity and collegiality which are aspects of rapport management (Spencer-Oatey, 2000c).

Sharing the same language or in-house jargon creates a sense of belonging in the community through active involvement (Wenger, 1998a, p. 173). This CofP had its own 'language' or technical jargon specific to the sector - construction site jargon and CofP specific jargon - as negotiated shared repertoire, usually a three letter acronym, which made no sense to outsiders. To protect the identity of the company, I can present only one example of CofP specific jargon, i.e. 'smoko' which is used by the staff members in this CofP when referring to the lunch room, in contrast with the dictionary definition which means "a work break for refreshment or to smoke a cigarette" (Bardsley, 2012; 2013, p. 348). Other general jargon shared among people from this construction workplace were $\mathrm{BCl}$ (Buyer Created Invoice), MOJ (Ministry of Justice), DFA (Delegated Financial Authority), JDE (J D Edwards), DJR (Daily Job Record) and RT (the radio transmitter fixed in company vehicles which blue collar workers used to contact the office). For a new employee, becoming familiar with the language takes time and this manifestation of a shared language is one of the dimensions of a CofP which helps create a feeling of belonging. To illustrate what I mean, I refer to an example extracted from my field notes "I was first introduced to the word 'smoko' when one of the engineers said 'Let's go to the smoko'. I looked puzzled as I was unsure what the engineer referred to until Sheila told me that 'smoko' was the lunch room. She explained how the room got this name "staff members called this the smoking room because it was adjacent to an area where smoking is permitted" (FN 22 August 2011). After that introduction, whenever staff members mentioned 'smoko', I knew instantly what they 
referred to. The reason that I had failed to understand this shared language is that this jargon had developed locally within that CofP.

At Company NZ2, requests were made either face to face or via email. Verbal requests were not preceded by small talk, nor were requests to perform routine tasks made via email accompanied by a grounder (see Section 3.6.4). The request could be targeted upward or downward or made among equals. This was a shared practice and generally staff members complied with the requests made (see Section 7.4.2 for details). Emails sent in response to a verbal request were usually sent without any messages in the body of the email. As with Company NZ1, this is one way of instantiating one of Wenger's (1998a, p. 126) features of CofP, namely "certain styles recognized as displaying membership". From the dataset collected from this workplace, these emails usually contained an attachment without a greeting, an introductory comment in the body of the email or a closing.

When managers gave instructions to the blue collar workers, they were framed as commands: one of the managers was heard to say 'if you don't know, just put your head down and shut up', 'if you drink don't drive a company vehicle', 'remember to be courteous', 'you must look professional', 'go to CBD' and 'don't fuck up'. Usually these instructions were given verbally during the daily morning briefing before work began and afternoon debriefing at the end of the day.

Humour is considered a distinctive feature of the culture of a workplace and provides a way of characterising workplace interactions (e.g. Holmes \& Marra, 2002; Marra \& Holmes, 2007; Schnurr, 2005). Unlike Company NZ1 where humour was predominantly positive and supportive, at Company NZ2, a rather different reaction towards humour was observed. The following observation noted on 29 August 2011 is an example of how humour was taken the wrong way and indicated that tolerance of humour was rather minimal in this workplace. It is worth noting here that all three participants in this example were symmetrical in terms of power relationships. When Megan's computer screen failed, Sam's big computer screen was taken from his workstation and was replaced by a smaller screen. This incident happened when Sam was on annual leave. When Sam returned to work the following week, he was upset because he now had a small computer screen and didn't take this change in a positive manner. When he asked his work colleagues what had happened to his big computer screen, 
no one gave him a definite answer, which irritated him, so he went to see Megan to sort out this problem. Megan who was then using his big screen did not tell him the truth. Then Ted, one of his work colleagues who shared the same office as Sam sent a humorous email to all the staff.

Unfortunately, the email which he (Ted) told me afterward was intended to amuse his workmates and to relieve boredom from the job, backfired. This email was accompanied by a picture of Sam's workstation without a computer screen to provide a visual representation of the message in the email. What was meant as a joke targeted at Sam was taken negatively when one of the Divisional Managers demanded to know what had happened to Sam's computer screen. This caused some friction between the three of them when both Sam and Megan became upset with Ted. However, if Sam had played along with the joke (i.e. collaborative humour) the outcome would have been different. In other words, rapport was not managed in this case by acknowledging the face needs and sociality rights (SpencerOatey, 2000c) of those involved. This example provides evidence that the appropriateness of 'doing humour' seems to depend on the norms of what is considered acceptable ways of interaction in specific workplaces or CofP. This example also indicates that Sam's face needs (i.e. he was the centre of joke) and sociality rights (i.e. his entitlement to a big computer screen) were violated. When one Divisional Manager put a stop to the joke, it seemed to indicate that the humour executed was beyond the boundaries of what was perceived as acceptable behaviour within this CofP.

The shared discourse repertoire observed in this workplace provided evidence that participants had to behave appropriately in order to be accepted as a core member. Not understanding that humour is not always viewed positively can result in friction between work colleagues.

Participating in the shared repertoire enabled the staff members to develop an identity which helped create a sense of belonging to a CofP. As discussed, workplace harmony was contributed to by both the individual and the company. Individuals took the responsibility of contributing to the solidary relationship by means of morning tea shouts while the company's efforts in reinforcing and maintaining a positive atmosphere was evident when social activities were organised for the staff members. One example given in this section was 
offering staff members free tickets to a rugby match. Because the workplace culture observed in this workplace seemed to place importance on transactional work, the availability of physical spaces and the open layout of the office did little to promote social interaction among staff members.

In summary, the three dimensions that are used to define the staff members in Company NZ2 as a CofP are listed below:

- Jointly negotiated enterprise - their goal was to provide excellent customer service to the company's clients and upheld the company's values which were to strive for excellence and to display commitment to the job;

- Mutual engagement - the staff members interacted with each other regularly at weekly meetings and minimally during morning tea; and

- Shared repertoire - they shared common linguistic and non-linguistic practices such as specialised terminology, birthday celebrations and company events.

In the previous sections, I have presented a description of two companies in New Zealand and the justification for classifying them as CofPs. Now I will introduce the company in Malaysia and justify why it is considered a CofP. 


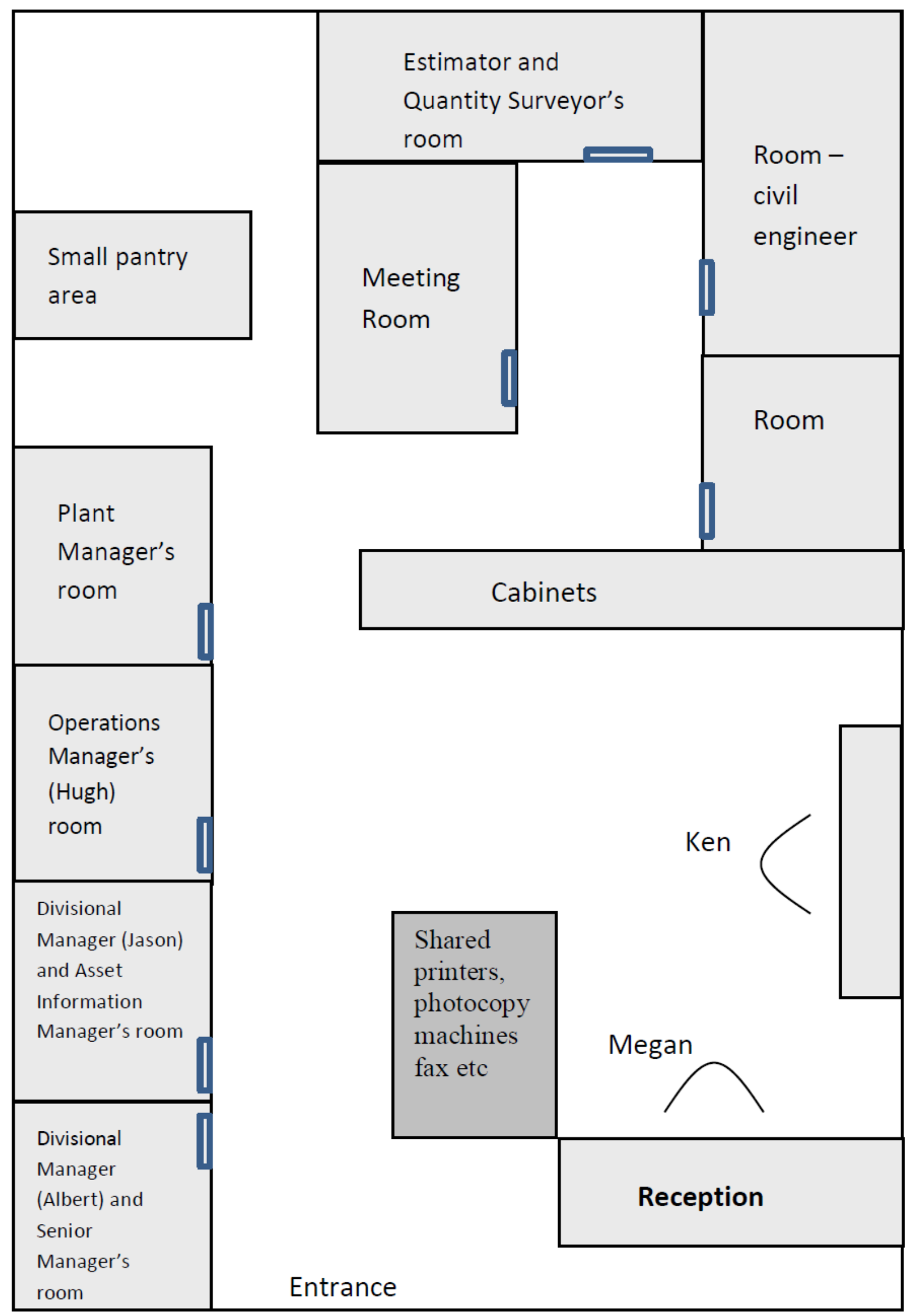

Figure 4.2 First floor of the main administration office building (Company NZ2) 


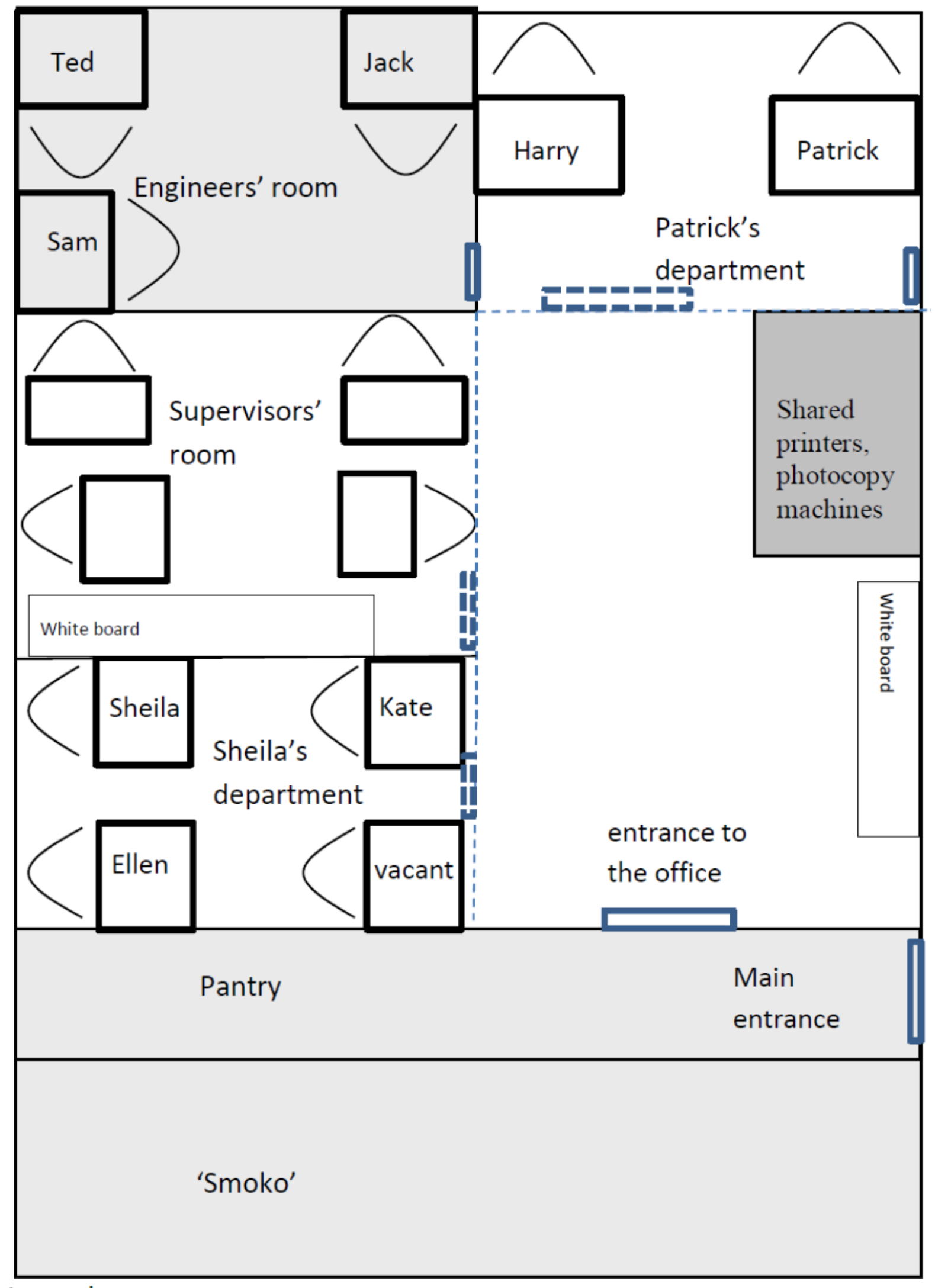

Legend:

Door to the room

Figure 4.3 Ground floor of the main administration office building (Company NZ2) 


\subsection{Company $\mathrm{M}$ as a community of practice}

Company M, as introduced in Section 3.3.1, was a Chartered Accountants firm. Although it operated under three different company names, it is an example of a constellation. Belonging to the same company, even though the staff members worked in different divisions - audit, tax, corporate finance, secretarial, accounting - they all had the same goals and contributed to the constellation as a whole.

It should be noted here that unlike New Zealand where English is the majority language, in Malaysia, English is considered a second language. However, in this company, as in many corporate companies, English is widely used in business transactions (Asmah, 1982). From my observation, all types of written communication (letters to clients, memoranda and emails) were produced in English while verbal communication was a mixture of English, Malay (the national language) and Tamil (a language spoken by Indians, one of the ethnic groups in Malaysia). All emails collected in this workplace were written in English. Meetings and discussions were usually conducted in English while a mixture of Malay and Tamil was used when conversing with the lower level staff (i.e. the despatch boys, the MD's driver and the tea lady).

In the following sections, I focus on the three criteria - jointly agreed enterprise, mutual engagement and shared repertoire - to analyse whether Company $\mathrm{M}$ qualifies as a CofP.

\subsubsection{Joint enterprise}

The joint enterprise of Company $M$ is evident in its company slogan "We are committed towards 'Corporate Social Responsibility'(CSR)". This shared goal was prominently displayed on a banner at the reception area. From the interviews conducted with the staff members, I understood that they shared the same view - the importance of 'giving back to the society'. They reported that they organised many charitable activities such as food sale, treasure hunts, 'Go Green' projects and garage sales, through the company's Sports Club (see Section 4.5.2) to achieve this objective. Many emails in my dataset illustrate the staff members' jointly agreed enterprise which relates to "mutual accountability" (Wenger, 1998a, p. 81). I provide just two examples here. The first email with the subject 'Jumble Sale by MAKNA' 
reads "In our continued efforts towards Corporate Social Responsibility (CSR), we have been called again by MAKNA to assist in their Jumble Sale." was sent by Geetha. The email gave details of the jumble sale and invited staff members to donate articles which were in good condition. All proceeds from the jumble sale went to MAKNA, a non-profit cancer organisation. The second email with the subject heading 'CSR Movie Blockbuster The Avengers' was sent by Sharifah inviting staff members to watch the movie and informing them that the proceeds from the sale of the movie tickets would be donated to the National Kidney Foundation.

In addition to the staff members' active involvement in the activities to promote the CSR concept as discussed above, they were also committed to the daily operations of the company. Staff members shared the same goal - to provide services that customers want and to provide better customer service - and this was evident when accounting projects were closed in a timely manner and each staff member was responsible to their respective clients. In other words, the staff members in Company M were aligned in their shared negotiated enterprise.

\subsubsection{Mutual engagement}

I have discussed in Sections 4.3.1 and 4.4.1 the impact of physical spaces and different layouts on the facilitation of regular interactions in Company NZ1 and Company NZ2 respectively. I now examine the physical space in Company $\mathrm{M}$ and its contribution in making mutual engagement possible. Company $\mathrm{M}$ occupied two units on the fifth floor in one office block and the two units were linked together in a $U$ shape (see Figure 4.4). Although the Ushape structure connected all the various departments, the doors leading into the individual departments were always closed. The closed doors were starkly juxtaposed with the open layout plan within each department. Desks were positioned in two rows with staff members facing each other. This open-plan layout reminded me of staff rooms for teachers in local schools in Malaysia which facilitated interactions between the teachers. Although this layout enabled staff members to have conversations with each other freely as shown in the discussion in Section 4.4.1 and Wilson's (2011) research, the office was, surprisingly, generally quiet. 
From the interview I had with the CEO and HR Manager, I understood that the open plan concept was designed to promote a 'family friendly environment' in the workplace. This concurs with Fletcher's (2011, p. 28) view that the person who has the power "makes decisions about spatial configuration and the allocation of space within the office". Although the open-plan layout and U-shape structure of the office might be expected to have a major influence on discourse strategies and communicative norms in this workplace, in a culture where hierarchy and status were given prominence, as in Company $M$, the open plan concept which was intended to promote a 'family friendly environment' seemed to have the opposite effect. For example, it seemed that staff members shared an understanding that small talk was unacceptable during office hours. I wrote in my field notes "small talk and humour were not common practice during office hours. Conversations that broke out occasionally were strictly work related. Staff members did not crowd around each other's workstations to have social chats. They seemed to be too busy to have social chats with one another during working hours" (FN 18 May 2012). Staff members from different departments only interacted when the need arose. This observation is somewhat similar to what I noted in Company NZ2 but in strong contrast to Company NZ1.

Meetings provided an avenue for regular interaction. As with Company NZ1 and Company NZ2, meetings in Company M were crucial to align business goals and objectives (Wenger, 1998a). From the interviews conducted and my observation, only senior staff members of the organisation hierarchy had a regular a bi-monthly meeting with the Managing Director which were fixed on Wednesdays at 9.00 am. During these meetings, each HOD gave an update of their individual departments and other important issues such as staffing matters were discussed. The meetings were usually chaired by the CEO and lasted about two hours or longer if needed (for example when there were serious issues that needed to be addressed). As in Company NZ1, the agenda was determined by the CEO and as the chairperson, he controlled the direction of these meetings. Usually the HR Manager, under the instruction of the CEO, sent a meeting reminder to all senior staff members via an email, and the email clearly specified that attendance was compulsory. This is one strategy for instantiating of power at work. 
Unlike the senior staff members in the hierarchy who had regular bi-monthly meetings, HODs did not have regular meetings with subordinates in their departments but had face to face discussions with them on an ad-hoc basis. For example, the HR Manager reported that it is impossible to have regular meetings due to the job demands of her subordinates. She usually had a short morning briefing with all her subordinates before work began at $8.30 \mathrm{am}$. Although formal meetings were not normally held with those lower in the organisational hierarchy, these morning briefings and the company's regulation that staff movements during office hours were recorded illustrated the rigid and relatively strict management imposed in Company M.

The staff members shared an understanding of what counted as appropriate behaviour on the day the management meeting was held. From my informal chats with the receptionist, I was informed that staff members studied the facial expressions of their respective HOD to determine if the HOD meetings were normal or unusual. If the HODs were happy at the end of the meeting, she said that it was a sign that the meeting went well. On the contrary, if they did not look happy, it was a sign that every staff member had to behave well to avoid being shouted at, reprimanded or scolded in public. It also meant that they had to avoid making mistakes on that day. In other words, it was expected that staff members 'read between the lines' and understood what appropriate behaviour they should display. This shared understanding not only marks the difference between a peripheral and a core member but it also saves the staff member from being reprimanded in public which could result in face loss (both quality face and identity face) (Spencer-Oatey, 2000c). It also signals the in-group non-communicative repertoire (i.e. the ability to read facial expressions of superiors) that core members develop over a period of time.

As in Company NZ1 and Company NZ2, meetings in Company M fulfilled one of the criteria of CofP, namely mutual engagement. Meetings also functioned as a means of 'asserting power' in this workplace. Emails sent as a reminder show how top management 'do' power by stating that attendance was compulsory, implying that attending these meetings was a priority. 


\subsubsection{Shared repertoire}

As in Company NZ1 and Company NZ2, Company M developed its own distinctive practices which characterised this CofP. Some of these practices were jointly negotiated while others were determined by those higher in the hierarchy of the organisation. These shared practices included greeting rituals, celebrations, the use of jargon and ways of making requests.

In the Malaysian context, in a culture that places great emphasis on titles and forms of address, the use of first name alone seldom occurs in asymmetrical relationships. It was a cultural practice in this company that members of the lower hierarchy of the organisation addressed their superiors formally in both written and verbal communication. Because I was born and educated in Malaysia, this localised practice is familiar to me although this company was new to me. My observation was also confirmed by other studies conducted in Malaysia (e.g. Abdullah, 2003; Alsree, 1997).

In Company $\mathrm{M}$, different levels of staff members used different forms of address when addressing their superiors or colleagues. According to the CEO, staff members in this workplace address their superiors according to the appropriate titles. For example, the HOD interviewed said he addresses the CEO as 'sir', 'boss' or 'En. ${ }^{16}$ Amir' and addresses the other HODs by first name. Generally, the staff members in this workplace addressed each other according to their level or status in the company. The CEO added that the practice of using titles in this workplace is merely a form of respect and does not suggest a 'master-servant' relationship among the employees. This formality was also evident in emails. The common greeting used was 'dear' followed by the recipient's 'title + name or surname'. Emails were usually signed off 'regards + first name'. No one signed off with the initial of the first name, a notable contrast with the practice observed in Company NZ1. See Section 6.5 for the discussion of the analysis of the use of greetings and closings in emails. The knowledge to employ this localised practice is one aspect which separates the peripheral and marginal members from the core members.

\footnotetext{
16 'En.' is an abbreviated version for 'Encik', a Malay equivalent of Mr.
} 
As in Company NZ1 and Company NZ2, celebrating staff members' birthdays was also a common practice in Company $\mathrm{M}$, one of the criteria of a "shared repertoire" (Wenger, 1998a). I was informed that birthdays were celebrated every two months, and not on the actual day. Unlike the New Zealand culture where the birthday person gives a 'shout' on his/ her birthday, birthday celebrations in this workplace were initiated by the management. These birthday celebrations were organised by different departments on a rotation basis and were usually held in the last hour of the working day. Having the celebrations at the end of the day gave the staff members an opportunity to mingle casually with one another, a rare sight during working hours in this workplace.

According to the HR Manager, the company tries to promote a social and family environment for the employees in line with the company's mission and vision. She said that the company formed an in-house club called the Sports Club with the main function of organising social events such as the company annual dinner, annual company trip, fund raising activities and sports related activities for the staff members. As the club was initiated by the management, one of the Directors of the company (also one of the managers) was appointed as the chairperson. From the interviews conducted, it was reported that the club was successful in developing social relationships amongst the staff members through the various social events and sports activities organised. The club's presence was viewed positively by the staff members.

Social events as mentioned above provided opportunities for this CofP to create a sense of belonging together. It also functioned as an avenue to build solidarity among staff members. It is worth noting that although the Sports Club was an initiative from top management, staff members responded positively to the announcements/ emails from this club. Clearly the positive response from staff members who were interviewed showed that rapport (SpencerOatey, 2000c) was established.

As with Company NZ1 and Company NZ2, this company had its own in-group jargon which an outsider could not understand. In this CofP, the word 'hijack' meant the act of intercepting phone calls. As an outsider when I heard it for the first time, I was puzzled, but the meaning of the word in context became clear to me when I spent more time at the reception area. By positioning myself at the reception counter, I also noted that the standard 
greeting used when answering external phone calls was 'Hello + [company name]'. In Company $\mathrm{M}$, all external phone calls went through the receptionist in the first instance. These calls were first screened by the receptionist and then re-directed to the respective recipients. By introducing the company name to callers, the receptionist helped create a sense of identity for the company.

On work related issues, instructions were often given by the CEO and the HOD to junior staff members. On the basis of interviews conducted, staff members preferred to give instructions via email because these emails were a record. These instructions were usually realised in the imperative form and unmitigated, with verbal instructions direct and explicit. Examples such as '[name of staff], do this'; 'follow up with the client on this matter' and 'please call [phone number] for me' were heard during my observation period. Sometimes the declarative form was used as one manager was heard to say 'I need you to go to the client's place tomorrow morning to send this cheque'. These instructions were usually not followed by a 'thank you'. While in other situations, this might be considered unacceptable and possibly labelled as rude, in this workplace, it was a shared understanding that these instructions were part of their routine tasks. This shared negotiated feature characterised Company M's workplace culture.

Only emails sent by the Sports Club used informal and non-institutional language. This informality is in marked contrast to transactional emails related to routine tasks (see Sections 7.4.3, 7.5.3 and 7.6.3 for examples of emails). Emails sent by the Sports Club always created a light mood and all staff members interviewed said they look forward to receiving such emails which were usually written in colourful fonts and/ or accompanied by relevant pictures, images and emoticons to suit the subject. The shared repertoire observed in this CofP helped create a collegial atmosphere.

Unlike Company NZ1 where collaborative humour was employed as a means of reinforcing solidarity, in Company $\mathrm{M}$, jokes were usually initiated by the top management (confirmed by staff members during interview sessions). When a joke was made, it seemed that it was inappropriate for a subordinate to respond, especially in a culture where asymmetrical position is greatly emphasised. Previous research confirmed this observation (Schnurr \& Chan, 2011). To quote an example, Amir, the CEO, sent an email to his secretary, Priya, 
informing her of a crack in one of the toilet seats in the male toilet. Amir described the consequences a staff member would experience as the result of sitting on a cracked toilet seat. Although the description was phrased in a comical and humorous manner, there was no evidence that this humour was taken up. This could possibly be attributed to the culture of this workplace supporting the notion of the "ethnicised CofP" introduced by Schnurr, Marra and Holmes (2007, p. 726) which refers to a workplace where staff members behave in "ways that are in accordance with the norms" and values of their "ethnic identities".

Another normal way of doing things observed in this CofP was how emails were sent in response to verbal requests. Unlike Company NZ1 and Company NZ2 where emails responding to requests were sent without any messages in the body of the email, staff members in Company $\mathrm{M}$ wrote a complete email. They began the email with a greeting, wrote a sentence to explain why the email was sent and signed off the email. In an interview with Kamal, he explained that it would be deemed as rude or disrespectful if he sent the reports without any acknowledgement. He added "that's not the way we do things here". From this interview and as confirmed by other interviewees, it is clear that this CofP had established a certain style of writing emails, in line with one of Wenger's (1998a, p. 126) features of CofP, namely "certain style of displaying membership".

It is important that staff members know what should and should not be done. The shared repertoire observed in this workplace provided evidence that participants should behave according to what is perceived to be a localised practice in order to be accepted as a core member. Using formal address forms to address work colleagues is an instantiation of respect in a culture where status and age are factors which are given important considerations. This localised practice and the use of specialised terminology (e.g. hijack) were negotiated shared practices which helped create bonding and construct solidarity among the staff members. Birthday celebrations and social events (i.e. charity sale, company annual dinner, a movie treat, etc.) organised by the company were indications of the company's effort to reinforce and maintain a positive atmosphere which promoted solidarity in the workplace. Unlike the New Zealand culture where celebrating birthdays is an individual's effort to cement solidarity, in the Malaysian workplace, the act of celebrating staff members' birthdays was initiated by the top management and the precise date for the celebration was selected by them. 
In summary, three dimensions that are used to define the staff members in Company $\mathrm{M}$ as a CofP are listed below:

- Jointly negotiated enterprise - their goal was to provide a range of consultancy service and expertise that customers want and better customer service to the company's clients;

- Mutual engagement - the staff members had regular interactions with each other at weekly meetings, birthday celebrations and social events organised by the top management; and

- Shared repertoire - they shared common linguistic and non-linguistic practices such as specific terminology, formal address forms in both written and verbal communication and appropriate behaviour to display during working hours.

In Sections 4.3, 4.4 and 4.5, I have demonstrated that in all three workplaces, the jointly negotiated enterprise was to provide excellent customer service to the company's clients. In addition, staff members interacted regularly during staff meetings and birthday celebrations, fulfilling the criteria of mutual engagement. A number of features of shared repertoire of the different CofPs such as regular greeting rituals, preferred ways of making requests, the types of greetings and closings used in emails, and the use of jargon and terminology at the workplaces were also evident.

In the next section, issues related to becoming a member of a CofP and their challenges are discussed. It is important that new members are inducted into the CofP so that they move from being a peripheral member and become a full participant. The challenge is two pronged: newcomers face the challenge of being inducted into the CofP; and core members distinguish themselves from others in order to retain their core membership and identity. 


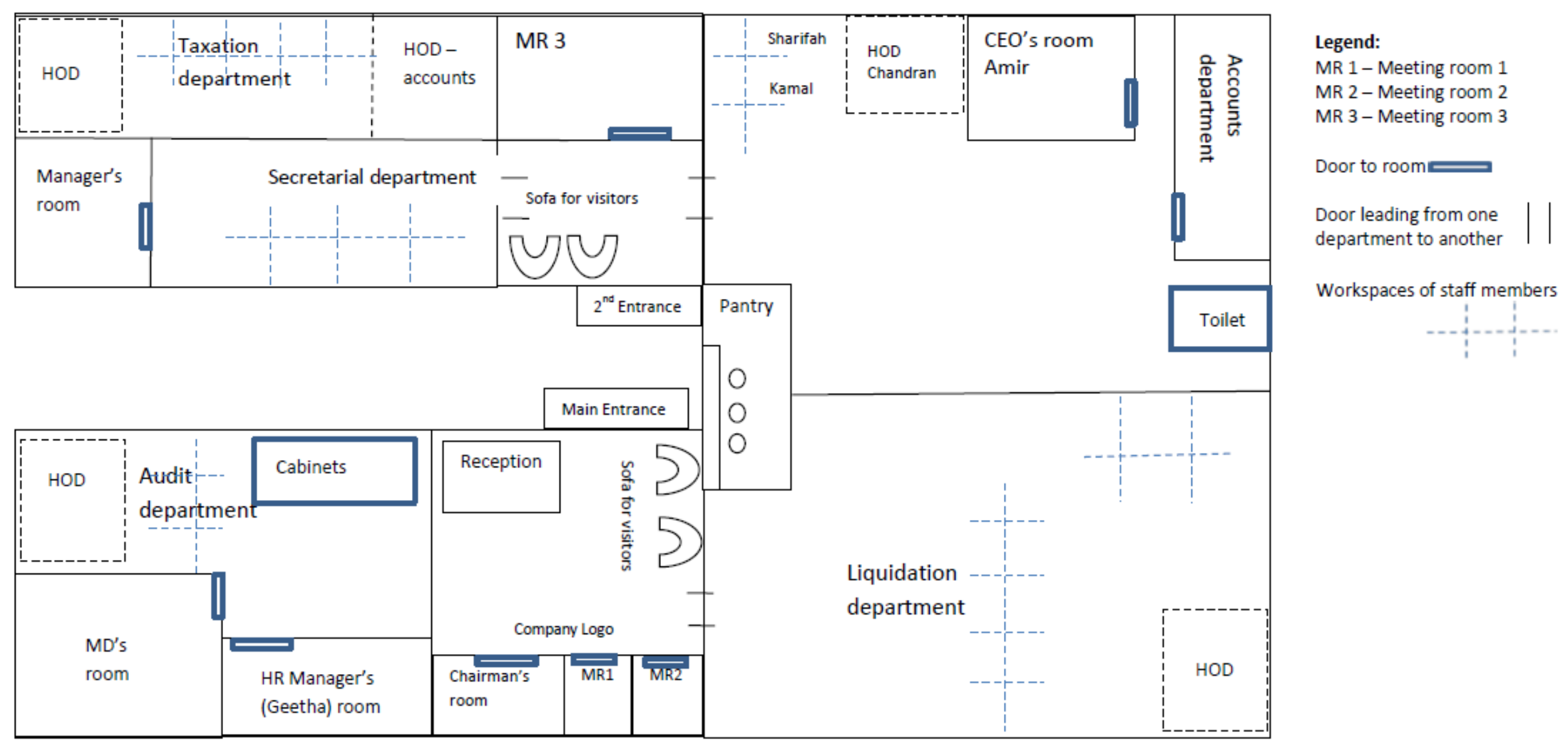

Figure 4.4 Layout of office (Company M) 


\subsection{Becoming a member in a community of practice}

As discussed earlier, one of the main challenges of CofP is to be accepted as a core member. So how does a newcomer learn and become a core member of the CofP? The process of becoming a member in any CofP requires the newcomer to learn the appropriate behaviours, both verbal and non-verbal, that characterise the group and differentiate it from others. Lave and Wenger (1991, p. 29) address this concern by introducing a process they label as "legitimate peripheral participation". According to Lave and Wenger (1991) this process of learning is through an engagement in appropriate practice, i.e. apprenticeship. They state that learning is a situated activity and that people learn by participating in an activity. Newcomers progress from being a peripheral member to a core member by participation through practice under the guidance and tutorship of the old timers of a CofP so that learners are given limited participation in an actual task and a certain degree of responsibility so that they can learn without undue pressure. These learners are said to be on an inbound trajectory, ready for full participation in the CofP.

Lave and Wenger (1991) refer to five case studies of apprenticeship and describe which is more or less successful, i.e. the process of learning in practice and the meaning of attaining full participation in the CofP. They cite the examples of Vai tailors and Yucatec midwives as more successful apprenticeships because the trainees were given the opportunity to increase their participation gradually in the practices within the community. In contrast, the apprenticeship of the butchers was less successful because an apprentice was allowed to learn one task at a time. In addition, an apprentice was only allowed to move to the next task if the previous apprentice left. In other words, the trainees were not allowed access to the entire practice necessary to award them full participation in the CofP. One point worth noting about learning is the influence of the "physical layout of a work setting" (Lave \& Wenger, 1991, p. 78). Using the example of the butchery apprenticeship, Lave and Wenger (1991, p. 78) claim that trainees learn more "from observing others and being observed".

According to Wenger (1998a), the concept of legitimacy is related to the forms of membership and the trajectory of the person in question: full participation, peripheral participation and marginal participation. A full participant is on the insider trajectory and maintains his/ her membership through participation. A peripheral participant may be on an 
inbound trajectory or a peripheral trajectory and has partial participation. A newcomer may be on an inbound trajectory and has the prospect of becoming a full participant. Marginal participants are on an outbound trajectory and their partial participation is considered a barrier to full participation.

In the workplace, newcomers do not always have a choice. They have to learn the practices of the community (i.e. workplace practices) in order to become full participants. Learning in a CofP is ultimately about legitimate peripheral participation. In other words, newcomers are put in a safe environment in which if they make mistakes, they have the opportunity to re-learn and practice. However, Wenger (1998a) stresses that access to a safe environment where a mistake is permissible is not always available:

On the school playground, the unmarked but sharp boundary of a clique can be a cruel reality, one for which well-meaning parents and teachers are of little help. The nuances and the jargon of a professional group distinguish the inside from the outside as much as do certificates. Not having the style and the connections can be as detrimental to an ambitious employee as the lack of a degree from a major business school. (Wenger, 1998a, p. 104)

When someone new joins a workplace, he/ she needs to learn the cultural norms of the workplace and the appropriate behaviours in order to gain acceptance into the CofP. Learning is inevitable if the newcomer wants to be accepted as a core member. In my study, newcomers not only needed to learn the appropriate behaviours, they also needed to learn the acceptable way of writing email requests that the CofP had already established. The aim is usually to gain cooperation from the members of the CofP rather than creating negative reactions from the recipients. So, peripheral members must learn to write their requests in ways that are both appropriate and acceptable in the CofP in order to gain full cooperation.

In some workplaces all newcomers are required to complete a period of formal training. For example, a training program for customer-service representatives (CSR) at a call centre (Brannan, 2007) and training for claims processors at an insurance company (Wenger, 1998a). In Company NZ1, there was no formal training program for new employees. Instead the CEO drew up an induction schedule, a process of training which involved all the staff members, where each team member spent about one hour to brief the newcomer on their respective job and responsibilities. 
The training process for a newcomer in Company NZ1 was quite similar to that of the apprentice Alcoholics Anonymous (A.A.) member in Lave and Wenger's (1991) case study, who attends a few meetings each week and spends time with others in the community to learn their practices and identity. At these meetings the newcomer learns the tricks of old timers of how to tell success stories of becoming sober and remain in that condition in order to be accepted in the A. A. community. Lave and Wenger (1991, p. 82) state that "telling an A. A. story is not something one learns through explicit teaching. Newcomers are not told how to tell their stories, yet most people who remain in A. A. learn to do this." Similarly in Company NZ1, the newcomer went through a training process which was similar to an onthe-job training. To illustrate this process, I refer to John, the newcomer. He joined Company NZ1 as a Relationship Manager - Events, two weeks after I started my research project. His role was to market all the events that Company NZ1 organised. Although he had had many years of experience in the field of marketing, in order to be a full participant in this CofP, he had to learn the practices of this community. Instead of a meeting with the whole group, as with the A. A., John was paired with all staff members, in turn, on different days for a one to one discussion session. During the discussion sessions, John learned the different job responsibilities of each old timer and the specific events that the old timer was in charge of. The purpose for this sort of arrangement was to enable John to move from an inbound trajectory to becoming a full participant in the CofP. Like the apprentice A.A. member who was not taught explicitly how to tell an A. A. story, John was not 'taught' how to market the events. He had to learn how to do so according to the expectations from the old timers and the workplace. To become a full participant, John had to demonstrate that he had become familiar with the specific job requirements and possessed the right knowledge to attain full membership. Once he had achieved those qualities, he would be fully integrated as a core member in the CofP.

Unlike Company NZ1, Company NZ2 arranged a formal induction programme for newcomers (interview with one of the Divisional Managers). Usually it was a one-day orientation session where the newcomer was given a briefing of the company's history, a tour of the office plant and an introduction to the company vision, mission and values. The formal induction programme illustrated power asserted from top management. Newcomers must comply with the rules and regulations stipulated and the objective of the induction 
programme was to facilitate learning. As the company emphasized health and safety, staff members were regularly sent for refresher training programs on this aspect. Some examples of training programmes were the 'First Aid' course and the 'How to handle equipment safely' course. In addition, in line with the company's policy on health and safety issues, it was mandatory for all staff members to attend these refresher training programmes. Noncompliance could have had negative repercussions.

Like Company NZ1, the Malaysian workplace, Company M, did not have any formal training programme for new employees. However, according to Geetha, the HR Manager, training plays an important role in the professional development of staff members. Unlike large organisations which organise in-house training programs, Company M sent staff members who required specific technical training courses to external providers. She also said that there is no formal induction program for newcomers due to the small size of the company, and perhaps because of the culture that the company practises. Induction programs were conducted on a one-to-one basis. This was in line with the company's practice, i.e. to promote a family friendly environment. When a newcomer reported to work on his/ her first day, he/ she was given a briefing on basic information by the HR Manager. Under a recently introduced peer concept, a newcomer was given a tour around the office and was introduced to the existing staff members by the CEO's secretary. The newcomer was then paired up with a senior staff member with the purpose of shadowing the senior staff for a period of two weeks. During these two weeks, the newcomer was given on-the-job training so that he/ she learned the technicalities required to perform his/ her job. This informal training meant that the newcomer was provided with an established shared practice to draw upon. Ultimately the newcomer learned and moved from being a peripheral member to a core member in this CofP. This process of attaining full membership and integration into the CofP mirrored that of the Vai tailors and the A. A. apprenticeship course (Lave \& Wenger, 1991). In addition, the open plan layout of the work setting also facilitated learning. Because the newcomer sat together with the old timers, he/she could also learn through observation. By observing what the old timers did, the newcomer learned what to do and what not to do. In other words, the newcomer became familiar with the shared repertoire that the community had developed. 
This section has explored the process of legitimate peripheral participation in three different workplaces. In both Company NZ1 and Company M, there was no formal training program organised for newcomers. The task of the newcomer was to learn on the job so that he/ she was able to produce competent performance. Newcomers learned the scope and complexity of the job as well as the shared repertoire developed by the community by way of participating in the task. The learning in practice eventually moved the newcomer on an inbound trajectory forward from a peripheral participant to a full participant. In contrast, a formal and structured induction program was designed for all new employees who joined Company NZ2. Newcomers learned the do's and don'ts in particular practices related to health and safety, so that they gradually became old timers. These practices were not negotiated and newcomers had to comply in order to become core members in the CofP. A formal induction programme such as the one conducted in Company NZ2 is an example of instantiation of power and control.

\subsection{Conclusion}

This chapter has covered three things. First, it has considered the perspective of physical space and spatial location of workplaces and their impacts on features of a CofP. Second, it has provided insights into the distinctive workplace culture which was developed in three different workplaces or CofPs through the three criteria: joint enterprise, mutual engagement and shared repertoire. And third, it has discussed how a newcomer becomes a member of a CofP through the process of legitimate peripheral participation within a workplace.

The concept of CofP has proven to be a suitable framework in order to highlight the unique characteristics of the three different workplaces (CofPs), and to compare their communicative practices. The discussion above shows that each CofP established different linguistic and non-linguistic practices which formed the basis of workplace culture. Each CofP has developed its own distinctive features such as its own way of dealing with other staff members, the acceptable manner in which email is written and the degree of tolerance towards humour in the workplace. My data has also illustrated that what is considered polite and appropriate practices in one CofP may be considered inappropriate and even 
impolite in other CofPs (Spencer-Oatey, 2000c). Through the discussion of physical space and proximity, I have shown that the use of space influenced the interaction of staff members in each workplace differently. While the open layout in Company NZ1 facilitated and promoted social interaction, the staff members in Company NZ2 and Company M appeared to be unaffected by it.

Although the discussion above showed that the three workplaces had formed a CofP in their own terms, it is important to remind ourselves of the following advice:

Practice, by its very nature, involves a relation to the world: it looks outward. Relations among participants within a community of practice are intricately tied to relations beyond the community of practice, and to the community's joint construction of its place in the wider world. (Eckert \& McConnell-Ginet, 2007, p. 28)

The advice is a good reminder that regardless of the factors which contribute to the formation of the CofP, the shared practices developed in a CofP will constantly be negotiated due to the influences from external forces. Having demonstrated why the three workplaces should be considered a CofP, the communicative functions of emails in each CofP is examined in the next chapter. 


\section{Chapter 5: Communicative Functions of Emails}

\subsection{Introduction}

This chapter reports the results of a survey administered to a total of 67 participants in the three workplaces and the follow-up interviews conducted with those who agreed to be interviewed (refer to Section 3.5.3 for details of interview methods). The email questionnaire and follow-up interviews were designed to investigate people's reported use of email, their email practices as well as their attitudes towards this technology. The findings obtained from the questionnaire and interviews were then compared to the actual email data collected.

This chapter is organised as follows. First, I describe the participants' reported use of email in the three workplaces. Then I give an overview of Speech Act Theory (Searle, 1969) which provides a framework for classifying the emails according to their communicative functions. Next I discuss the communicative functions of emails for each workplace. The chapter ends with a discussion of the similarities and differences in the communicative functions across the three workplaces.

\subsection{Email usage in three workplaces}

In this section I discuss the participants' reported usage based on the questionnaire I administered and interviews I conducted at each workplace. The data presented are useful for two reasons. First, they offer an insight to what the participants said they did; and second, the data enable the researcher to make a comparison between what the participants reported they did and what they actually did with reference to email communication. The data from each workplace is discussed in turn. 


\subsubsection{Email usage in Company NZ1}

Email has become an important tool of communication in Company NZ1 and participants reported that it takes up a reasonable amount of time in people's working day. Sixty percent of 15 staff members surveyed reported that there has been an increase in email usage. Table 5.1 shows the amount of time spent on email communication in the participant's working day.

Table 5.1 Amount of time spent on email daily by participants in Company NZ1

\begin{tabular}{lcc}
\hline Amount of time spent & \multicolumn{2}{c}{ Respondents } \\
\cline { 2 - 3 } on email daily & No & Percentage (\%) \\
\hline More than 2 hours & 5 & 33 \\
$1-2$ hours & 4 & 27 \\
30 minutes -1 hour & 4 & 27 \\
$0-30$ minutes & 2 & 13 \\
\hline Total & $\mathbf{1 5}$ & $\mathbf{1 0 0}$ \\
\hline
\end{tabular}

As indicated in Table 5.1, 87 percent of the participants surveyed reported that they spend more than 30 minutes in a working day on email, with more than one third of them spending over two hours daily replying emails. This result is similar to Waldvogel's (2005) research. Her study reports 86 percent of the staff surveyed reported that they spent more than 30 minutes a day on email.

In my study, 14 of those interviewed said that they attend to their email continuously. Two staff members, Andy, the CEO, and Symone, one HOE, said that they checked their email at home before coming to the office. Only one staff member, John, said that he allocates a specific time to check his email and this is within an hour of starting his day. He commented that email is a huge distraction and that he prefers to check his inbox after he has cleared the most important tasks of the day. He said 'if you are not careful, email will drive your day'. While a considerable amount of time was spent on emails, none of the participants reported that it is a waste of time.

Participants were asked to indicate the frequency of using emails for various communicative functions. In the questionnaire (see Appendix 5), I asked the participants to report on the frequency of use of email for 13 communicative functions in Section B. However, for the purpose of discussion in this chapter, I focus on seven relevant communicative functions from the questionnaire to match as closely as possible the 
categories discussed in Section 5.3. Table 5.2 and Figure 5.1 show the reported frequency of use of email for the following seven communicative functions: sending information, making requests, offering feedback, sending compliments, saying thank you, seeking advice and resolving conflict. For discussion purposes, I have grouped 'never' and 'seldom', and 'often' and 'always' together.

Table 5.2 Frequency of reported use of email in Company NZ1

\begin{tabular}{|c|c|c|c|c|c|c|c|c|}
\hline \multirow{3}{*}{ Communicative Functions } & \multicolumn{8}{|c|}{ Frequency of use } \\
\hline & \multicolumn{2}{|c|}{$\begin{array}{l}\text { Often and } \\
\text { Always }\end{array}$} & \multicolumn{2}{|c|}{ Sometimes } & \multicolumn{2}{|c|}{$\begin{array}{l}\text { Never and } \\
\text { Seldom }\end{array}$} & \multicolumn{2}{|c|}{ Total } \\
\hline & No & $\%$ & No & $\%$ & No & $\%$ & No & $\%$ \\
\hline sending information & 13 & 87 & 2 & 13 & 0 & 0 & 15 & 100 \\
\hline making requests & 7 & 47 & 7 & 47 & 1 & 7 & 15 & 100 \\
\hline offering feedback & 8 & 53 & 6 & 40 & 1 & 7 & 15 & 100 \\
\hline sending compliments & 8 & 53 & 3 & 20 & 4 & 27 & 15 & 100 \\
\hline saying thank you & 11 & 73 & 3 & 20 & 1 & 7 & 15 & 100 \\
\hline seeking advice & 3 & 20 & 10 & 67 & 2 & 13 & 15 & 100 \\
\hline resolving conflict & 0 & 0 & 5 & 33 & 10 & 67 & 15 & 100 \\
\hline
\end{tabular}

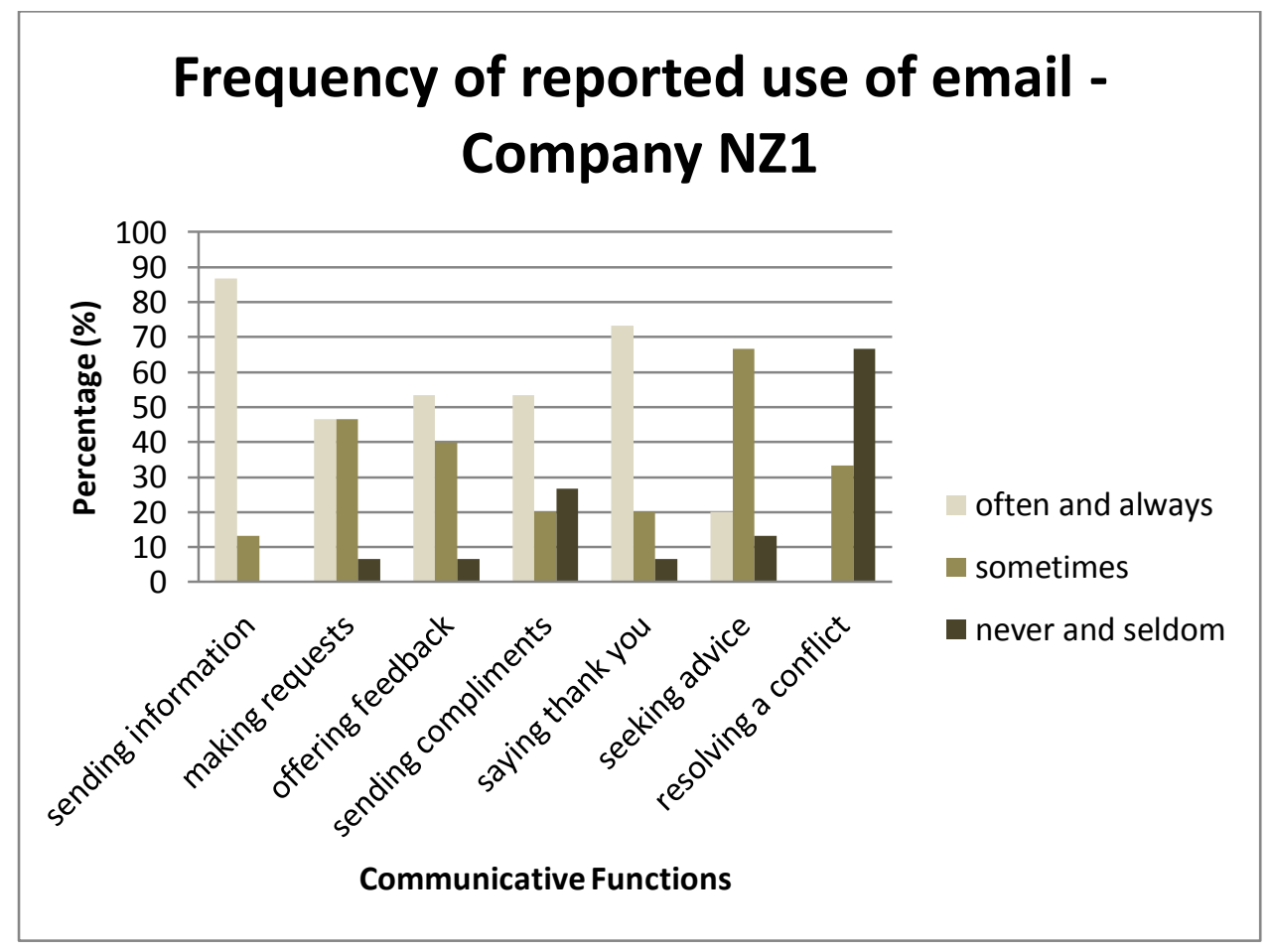

Figure 5.1 Frequency of reported use of email in Company NZ1 
The participants reported that they 'always' or 'often' use email to do the following: send information (87\%), send compliments (53\%) and say thank you (73\%). Email is 'sometimes' used for two purposes: making requests (47\%) and seeking advice (67\%). Slightly more than half (53\%) of the participants said that they 'often' offer feedback via email. Sixty-seven percent of the participants said they 'never' or 'seldom' use email to solve conflicts.

My findings indicate that emails were used for various purposes. The participants interviewed reported that email is excellent for sending information to a number of people so that everyone gets the same message at the same time. To quote an example, the CEO, Andy, sent an email as a reprimand to staff members who were involved in a car park space dispute. During an interview, she said that she chooses to write an email over other modes of communication because she wants the same message in the same tone delivered to the staff members at the same time. See Appendix 6 for the full email (Email 6.19 [SA31] NZ1).

In addition to sending information, staff members interviewed said that email is also useful when people need to keep a record of a conversation or discussion that took place. Another useful feature of email is that it assists in administrative tasks such as scheduling meetings and setting appointments. Everyone interviewed agreed that the shared calendar feature is very useful because the tool enables everyone to be aware of their colleagues' movement. This was especially important because the CEO, HOE and the Relationship Managers travelled to different parts of the country on a regular basis.

The fact that email can include attachments is viewed very positively. They said that emails are used very often to send information in the form of attachments following a face to face discussion. Such emails were usually not accompanied by any message or subject line. My analysis of the email dataset supports the participants' reports.

Furthermore, emails were extremely useful when they needed to arrange for meetings. They would send a 'meeting request' and this meeting would be recorded automatically in the recipients' calendar. Other uses of emails included:

1. sending newsletter to clients who were geographically dispersed in both the North and South Islands. 
2. keeping in touch with presenters all over the country which would otherwise be very difficult due to logistics problems.

3. maintaining relationships which had already been established via face to face

4. confirming account numbers to avoid mistakes.

Participants interviewed reported that the choice of using email over other modes of communication depends on various factors such as:

a. the topic/ message

b. who the recipients are

c. where the recipients are geographically

d. the number of people one is trying to reach

e. whether the message delivered needs to be received by all recipients at the same time

Although email has many advantages and staff members deemed it an effective mode of communication for the various communicative functions discussed above, generally staff members interviewed said that they will not use email for dealing with delicate and sensitive issues. This concurs with the findings from the questionnaire that staff members reported that they 'never' or 'seldom' use email to resolve conflicts (see Table 5.2). The CEO, Andy, said that staffing issues should never be dealt with via email. She considered that such matters are best dealt with face to face. Andy's opinion is consistent with the managers who were interviewed in Markus' (1994b) study. The managers in Markus' (1994: 519) study argued that "email was regarded as the primary medium of internal work-related communication, appropriate for any task that did not involve social, work-related interpersonal or personnel matters". Because staff issues can be delicate and may involve emotional feelings, email in this case is not a suitable medium to use. All interviewees agreed that email is not a suitable means of communication for conveying information that could be misinterpreted, misunderstood or taken the wrong way. Staff members also deemed email as unsuitable in the following situations as quoted by a few interviewees:

a. "When emails were going back and forth a few times but the issue was not resolved." 
b. "When you need an instant answer."

c. "When you know that the recipient is not very efficient in replying to emails."

How email was used by each staff member and the hours spent on it were also different, as discussed above. But the statistics show that the richness of email can also fulfil an interpersonal function such as sending compliments and saying thank you (cf Daft \& Lengel, 1984).

The next sub-section discusses the email usage in Company NZ2 and shows how email usage was managed differently from Company NZ1.

\subsubsection{Email usage in Company NZ2}

In contrast with Company NZ1, Company NZ2 was more heavily dependent on using emails as a medium of communication amongst staff members. Ninety-nine percent of the staff members interviewed said that email plays an important role in their job and the business as a whole. However, they said that email is strictly for business use and disciplinary actions would be taken against staff members who misused their work email. Approximately 50 percent of them reported that there had been an increase in email usage in the past year. Table 5.3 shows the amount of time spent on email communication in the participants' working day.

Table 5.3 Amount of time spent on email daily by participants in Company NZ2

\begin{tabular}{lcc}
\hline Amount of time spent & \multicolumn{2}{c}{ Respondents } \\
\cline { 2 - 3 } on email daily & No & $\%$ \\
\hline More than 2 hours & 8 & 38 \\
$1-2$ hours & 9 & 43 \\
30 minutes -1 hour & 3 & 14 \\
$0-30$ minutes & 1 & 5 \\
\hline Total & $\mathbf{2 1}$ & $\mathbf{1 0 0}$ \\
\hline
\end{tabular}

Almost half of the respondents reported that their main communication activities are dominated by email with 80 percent of them spending more than one hour on email exchanges. Table 5.3 shows that 95 percent of the respondents surveyed reported that they spend more than 30 minutes daily on email with 38 percent of those spending 
more than two hours daily on this technology. This result shows that staff members in this workplace spent a greater amount of time on email daily compared to the participants in Company NZ1 (see Section 5.2.1) and also in Waldvogel's (2005) research. The majority of the respondents said that they check their email inbox the moment they arrive at the office. As most them were out on field work, they were also able to access their emails via their mobile phones. They noted that it is important to clear their inboxes periodically due to the volume of emails received daily. This result mirrors that of Markus (1994b) who reports that her respondents who were managers remained logged on to email all the time and responded to email messages as soon they arrived into their inboxes.

My findings indicate that the staff members at Company NZ2 managed their emails differently from each other: some read their email the moment it arrived in the inbox; some glanced at the subject line when a new email alert popped on their screen to decide whether to check that email or not; and some allocated a certain time of the day to check their emails. However, all claimed that email is a distraction and can take up a lot of time if it is not managed efficiently. This view concurred with John's, a participant from Company NZ1. Although many who were interviewed feel that email took up a lot of their time, none of them reported that it is a waste of time.

Participants were asked to indicate the frequency of using emails for various communicative functions. Table 5.4 and Figure 5.2 show the frequency of reported use of email for the following seven communicative functions. 
Table 5.4 Frequency of reported use of email in Company NZ2

\begin{tabular}{lccccccccc}
\hline \multirow{2}{*}{\begin{tabular}{l} 
Communicative Functions \\
\cline { 2 - 10 }
\end{tabular}} & $\begin{array}{l}\text { Often and } \\
\text { Always }\end{array}$ & Sometimes & $\begin{array}{c}\text { Never and } \\
\text { Seldom }\end{array}$ & Total \\
\cline { 2 - 11 } & No & $\%$ & No & $\%$ & No & $\%$ & No & $\%$ \\
\hline sending information & 18 & 86 & 0 & 0 & 3 & 14 & 21 & 100 \\
\hline making requests & 14 & 67 & 0 & 0 & 7 & 33 & 21 & 100 \\
\hline offering feedback & 11 & 52 & 4 & 19 & 6 & 29 & 21 & 100 \\
\hline sending compliments & 8 & 38 & 10 & 48 & 3 & 14 & 21 & 100 \\
\hline saying thank you & 10 & 48 & 8 & 38 & 3 & 14 & 21 & 100 \\
\hline seeking advice & 9 & 43 & 11 & 52 & 1 & 5 & 21 & 100 \\
\hline resolving a conflict & 5 & 24 & 2 & 10 & 14 & 67 & 21 & 100 \\
\hline
\end{tabular}

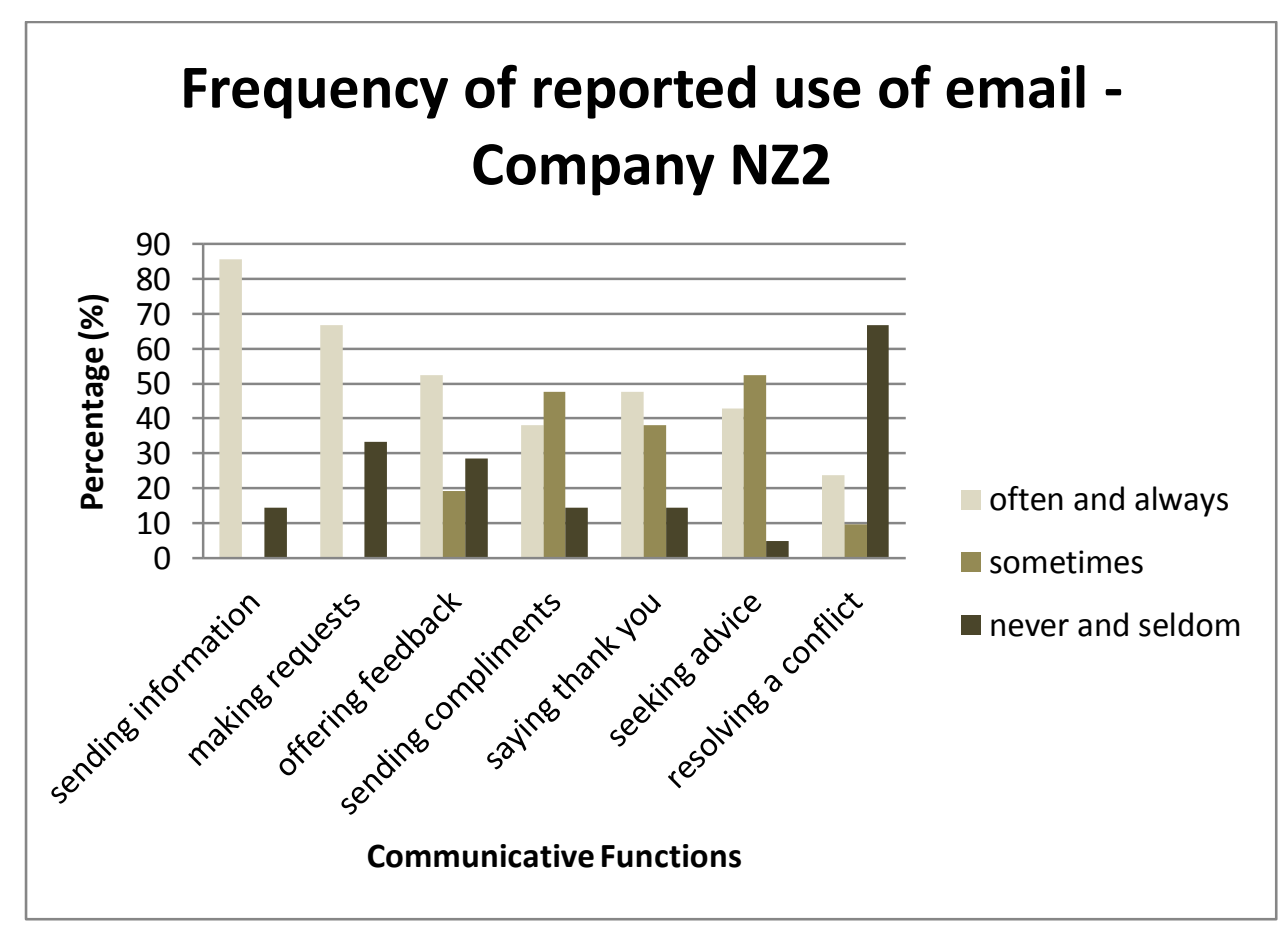

Figure 5.2 Frequency of reported use of email in Company NZ2

Participants reported that they 'always' or 'often' use email to do the following: send information (86\%), make requests (67\%) and offer feedback (52\%). Email is 'sometimes' use for two purposes: sending compliments (48\%) and seeking advice (52\%). Less than half of the participants said that they 'always' or 'often' use email to say thank you (48\%). In contrast with Company NZ1 where none chose the options 'always' and 'often' for 
resolving conflicts via email, 24 percent of the participants in Company NZ2 selected this option.

As in Company NZ1, email was reportedly used for various reasons. Ninety percent of the staff members interviewed said that email is used as an "ass covering" exercise which is very salient in their industry. Email is used to keep a record of conversations or discussions that had taken place between colleagues from different departments. When emails are sent between staff members of the same department, these emails serve three purposes - as a reminder to get things done, as a record of instructions requested and as a way to keep each other informed. Another important usage of email is to record minor staffing issues related to HR procedures and disciplinary meetings. Other uses of emails include:

1. letting people know that things had been done.

2. communicating with clients

3. communicating with people who did not provide a phone number

4. sending meeting invitations

As in Company NZ1, the reasons for the staff members' choice of modes of communication in Company NZ2 depended largely on a range of factors such as distance between writer and recipient, the number of recipients that the message needed to reach and the recipient. Unlike Company NZ1, nearly everyone interviewed in Company NZ2 said that there are no business situations for which email is inappropriate. This view mirrors that of the managers who were interviewed in Markus' (1994) research. The managers were of the opinion that "email was appropriate for all work-related communication" (Markus, 1994: 520). However, during the interviews, participants quoted a few situations where email is deemed unsuitable:

a. "When emails were going back and forth more than once".

b. "When an instant answer is required".

c. "When it is an urgent matter".

d. "When I needed a big favour".

e. "When things are uncertain or if there is any kind of confrontation involved". 
f. "When offering advice or opinions which need not be recorded".

g. "When I need to walk someone through a process".

h. "When I am angry or annoyed with someone".

In summary, staff members in Company NZ2 deemed that email is very important because of the nature of the industry: emails recorded the date of discussions and there is a trail of the historical development of discussions that had taken place. Therefore, email communication is viewed as critical in avoiding disputes or disagreements which may arise in the future and it is also one way of avoiding potential costly litigation procedures. In this workplace, contracts sent via email were deemed binding and any amendments to the contracts made via email were also given the same weight as any piece of evidence in the eyes of the law, a practice which concurs with the findings of two earlier studies. Mills and her colleagues comment that (2000, p. 69) "deletion of email records may be considered as destroying evidence and refusing to produce such evidence can result in the loss of a lawsuit". Also, Gains $(1999$, p. 90; 97) finds that the commercial emails in his study could "have a permanent legal status" because "they provide a unified permanent record of internal communications within the company, and they become a dated legal record of such communications whether they refer directly to specific insurance policies or policy holders".

The previous two sub-sections discussed how email usage was managed in two New Zealand companies (Company NZ1 and Company NZ2). In the next sub-section, I present a discussion of how email usage was managed in a Malaysian company (Company M).

\subsubsection{Email usage in Company $M$}

As shown in Table 5.5, participants from Company $\mathrm{M}$ made far less use of emails than Company NZ1 and Company NZ2. The data presented in the table suggests that the staff members spent less time on email usage in one working day. Seventy four percent of them reported that they spend less than one hour a day on email. This result seems to be at odds with the statistics where 87 percent of the staff members reported that their email usage had increased. Closer analysis of the questionnaires reveals that only staff 
members in the middle and senior management were greatly affected by email use. This observation was confirmed in the interviews conducted with the middle and senior level staff members. Table 5.5 shows the amount of time spent on email communication in the participant's typical working day.

Table 5.5 Amount of time spent on email daily by participants in Company M

\begin{tabular}{lcc}
\hline \multirow{2}{*}{$\begin{array}{l}\text { Amount of time spent } \\
\text { on email daily }\end{array}$} & \multicolumn{2}{c}{ Number of respondents } \\
\cline { 2 - 3 } More than 2 hours & No & \% \\
$1-2$ hours & 2 & 19 \\
30 minutes -1 hour & 11 & 6 \\
$0-30$ minutes & 12 & 35 \\
\hline Total & $\mathbf{3 1}$ & 39 \\
\hline
\end{tabular}

As indicated in Table 5.5, 25 percent of the participants surveyed reported that they spend more than one hour in a working day on email with 19 percent of those spending over two hours daily on it. This result which contrasts the findings reported in Sections 5.2.1 (Company NZ1) and 5.2.2 (Company NZ2) shows that staff members in this workplace spent less time on this technology.

All staff members interviewed said that they check their email inbox the moment they arrive at the office and attend to their email continuously throughout the day. Three senior staff members said that they check their email constantly. The CEO reported that "I am connected 24/7" and two others said they check their email even when at home. None reported that email is a waste of time.

Participants were asked to indicate the frequency of using emails for various communicative functions. Table 5.6 and Figure 5.3 show the frequency of reported use of email for seven communicative functions. 
Table 5.6 Frequency of reported use of email in Company M

\begin{tabular}{lccccccccc}
\hline & \multicolumn{9}{c}{ Frequency of use } \\
\cline { 2 - 11 } Communicative Functions & $\begin{array}{l}\text { Often and } \\
\text { Always }\end{array}$ & Sometimes & \multicolumn{2}{c}{$\begin{array}{c}\text { Never and } \\
\text { Seldom }\end{array}$} & \multicolumn{2}{c}{ Total } \\
\cline { 2 - 12 } & No & $\%$ & No & $\%$ & No & $\%$ & No & $\%$ \\
\hline sending information & 23 & 74 & 7 & 23 & 1 & 3 & 31 & 100 \\
\hline making requests & 14 & 45 & 10 & 32 & 7 & 23 & 31 & 100 \\
\hline offering feedback & 7 & 23 & 16 & 52 & 8 & 26 & 31 & 100 \\
\hline sending compliments & 7 & 23 & 16 & 52 & 8 & 26 & 31 & 100 \\
\hline saying thank you & 19 & 61 & 10 & 32 & 2 & 6 & 31 & 100 \\
\hline seeking advice & 15 & 48 & 13 & 42 & 3 & 10 & 31 & 100 \\
\hline resolving a conflict & 7 & 23 & 13 & 42 & 11 & 35 & 31 & 100 \\
\hline
\end{tabular}

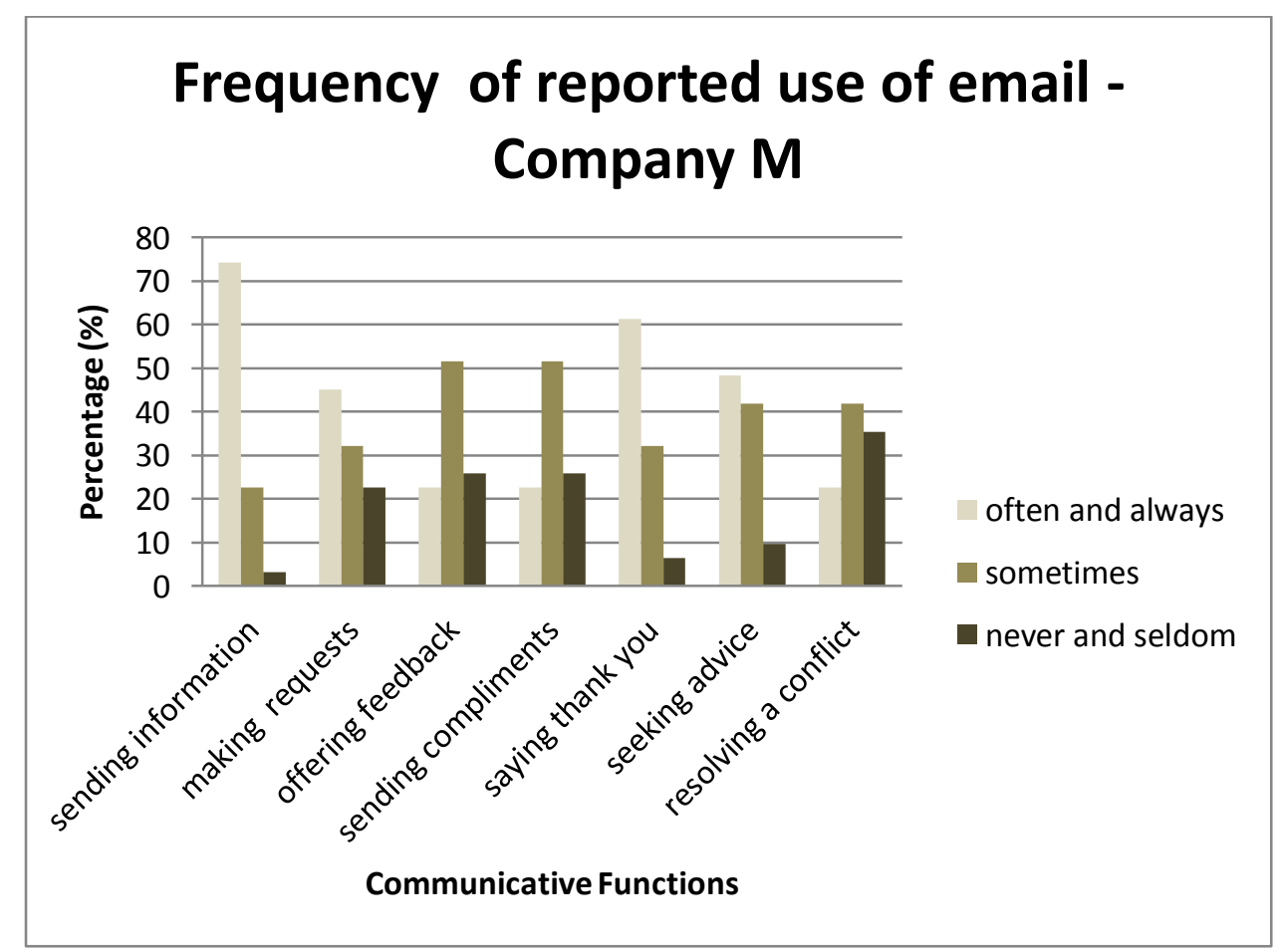

Figure 5.3 Frequency of reported use of email in Company M

Participants reported that they 'always' or 'often' use email to do the following: send information (74\%) and say thank you (61\%). Less than half of the participants said that they 'always' or 'often' use email to seek advice (48\%) and make requests (45\%). Email was reportedly 'sometimes' used for two purposes: sending compliments and offering feedback (52\% each). As with Company NZ2, 23 percent of the participants reported that 
they 'always' or 'often' use email to resolve conflicts. This is in contrast with Company NZ1 as none of the participants in Company NZ1 chose the options 'always' and 'often' for resolving conflicts via email.

Email was used for various purposes. All the staff members interviewed said that email is used for keeping records which is salient in their business. As in Company NZ2, email usage in Company $\mathrm{M}$ was also strictly for business. In an interview, Amir reported that staff members are aware that workplace email is mainly work related. Generally email is important for three reasons in Company $\mathrm{M}$ :

a. as a reminder to get things done by a certain due date;

b. as a record of information given by one individual to another; and

c. as a record of instructions given by top management to subordinates.

In addition, the CEO encouraged all staff members to keep a record of verbal discussions especially when the information could have a negative impact on the business. He said that he tries to inculcate the habit of recording all discussions (both internal and external) via email. In this company, email was also used to record minor staffing issues such as first warning notice on staff tardiness or other disciplinary matters. This could be a possible explanation for participants reporting that they 'always' and 'often' use email to resolve conflict. However, for major staffing issues, a formal letter would be issued as email is not accepted under the Employment Act 1955 in Malaysia.

According to those interviewed, there are no business situations for which email is inappropriate, a view which echoed those interviewed at Company NZ2. However, staff members interviewed said that they will not use email in situations when:

a. an answer is required urgently.

b. the answer required needs a lengthy explanation.

c. the writer needs to know the recipient's reaction to a favour requested.

In addition, due to strict regulations imposed by the Malaysian government, the following are two situations where email is considered not binding: (1) formalising a business deal which requires the client's signature; and (2) dealing with regulatory authorities. 
In summary, because email records the date of a discussion that took place and what was agreed between two parties, it is deemed salient in this industry to avoid legal disputes and costly litigation which might arise in future, an opinion shared by the participants in Company NZ2. Internally, emails were useful to keep track of deadlines and both superior and subordinates could use the emails for future reference. Unlike Western countries, emails do not have legal authority in Malaysia, a practice which contrasts with Mills et al.'s (2000) observation where the deletion of email is considered tampering with legal evidence. Reinforcing this view, the HR Manager in Company M pointed out that all disciplinary letters to employees have to be written on the company's letterhead and have to be handed directly to the employee concerned. In other words, emails are not accepted and do not carry any legal status in Malaysia.

In the previous sections, I have discussed the usage of emails as reported by the participants in the three workplaces. The following section presents a discussion of Speech Act Theory, a framework used for classifying the emails collected according to their communicative functions.

\subsection{Speech Act Theory}

In spoken language, one or more individual speech acts which perform certain functions contribute to communicative events. Similarly email messages can be considered communicative events and typically comprise a number of speech acts.

According to Speech Act Theory as developed first by Austin (1962) and later by Searle (1969), language is used to perform various acts such as giving instructions or information, apologising or advising in one communicative event. Each of these acts is called a speech act, i.e. an act that we perform through speaking, and it may convey more than one meaning. Speech acts have both a "literal" meaning and the intended meaning of the speaker who uttered the speech act, i.e. the illocutionary force. Because the speaker attempts to produce a certain effect on the hearer by the utterance, speech acts are considered intentional actions. To understand these concepts, Austin (1962, p. 120) proposes a theoretical base to classify the different kinds of acts that are realized in an utterance as follows (examples are given to illustrate the various acts): 
1. Locutionary act which has a literal meaning. An example is when someone says: 'It's hot in here'.

2. Illocutionary act which has a certain force in saying something. An example is that the person who says 'It's hot in here' means 'Open the door'.

3. Perlocutionary act which is the achieving of certain effects by saying something. An example is the act of someone opening the door in response to the person who says 'It's hot in here'.

Austin's (1962) primary interest is in the illocutionary act and illocutionary force. The force of an utterance depends on the speaker's intention and how it is realized linguistically. The varying degree of illocutionary force is considered as an interaction strategy. Therefore, the meaning the speaker intended for the hearer is located in the illocutionary force of an utterance. Expanding on Austin's (1962) work, Searle (1969) proposes five categories of illocutionary acts: representatives, directives, commissives, expressives and declarations. Representatives are propositions that are either true or false (Searle, 1976, p. 10). In other words, they state things as they are perceived to be. Directives are "attempts to get the hearer to do something" (Searle, 1976, p. 11). Commissives are illocutionary acts which "commit the speaker to some future course of action" (Searle, 1976, p. 11). Expressives express the speaker's psychological state regarding a particular situation such as thanking, congratulating, apologizing, deploring and welcoming. Declarations are the successful performance of the proposition. An example given by Searle $(1976$, p. 13) is "if I successfully perform the act of marrying you, then you are married".

However, this theory has been criticized because it is possible for an utterance to convey several meanings or to have a range of illocutionary forces. Taking the example above, the utterance 'It's hot in here.' could be interpreted at face value and be taken as an opinion of the temperature as a representative. It could also be interpreted as someone's request for another to open the door or as permission to open the door (as a directive). Because a speaker's intention may not be explicit, an utterance like the example above could give rise to several interpretations. In other words, the 
illocutionary force of a speech act is dependent on its context and an utterance may also be multi-functional.

Methods which have been used for classifying the communicative functions of emails are outlined below. Yates and Orlikowski (1992, p. 301) introduced the concept "organisational communication" and referred to it as "typical communicative practices occurring in organisations". They traced the development of the business memoranda genre which evolved from the business letter genre in response to the institutional and technological changes which had taken place so rapidly in the previous two decades. According to them, the genre may be applied to different types of organisational communication such as letters, memoranda, business proposals, shareholders' annual reports, financial reports or agendas for meeting which are characterised by communicative purpose, structural, linguistic and substantive conventions. They categorised all messages with an embedded message (messages that contained a previous message) as dialogues, and labelled messages with "no aside to individuals, no embedded message, no graphical element, no heading, no nonstandard elements, no opening and no sign-off" as memos (Orlikowski \& Yates, 1994, p. 556). However, Orlikowski and Yates' (1994) distinction between dialogue and memo proved not to be relevant for analysing my dataset of emails. Hence, I did not adopt their classification method.

Sherblom (1988, p. 46), who analysed 157 email files, coded his corpus according to message functions and created the following categories: "requests for information, providers of information, influence attempts, administrative items (such as meeting announcements, requests to meet, minutes of meeting), personal and social remarks and miscellaneous (such as acknowledgments, reminders)". This method is quite similar to Speech Act Theory.

Another researcher, Rice (1997) proposes a classification system based on an overlap of functional and genre types to categorise 200 self-selected email memoranda. The categories are "announcements, requests, explanations, replies, notices, confirmations, reminders, instructions, suggestions, acknowledgements, invitations, offers" and others 
(Rice, 1997, p. 10). Rice's classification system has much in common with Searle's speech act categories.

Waldvogel (2005) adopts Searle's (1969) taxonomy of speech acts with some modifications to classify a corpus of 515 emails collected from an education institution and a manufacturing plant. Her classification system based on speech acts is as follows:

- Information giving, which corresponds to Searle's representatives.

- Directives, which include all speech acts where someone is being asked to do something. This speech act only partially corresponds to Searle's category of directive because information seeking questions and invitations were excluded.

- Information requests, which covers questions seeking information, advice or an opinion.

- Invitations, which are a subset of Searle's directives, seen to be of more benefit to the recipient.

- Commissives, which commit the speaker to future actions such as promising or offering.

- Expressives, which commit the speaker to the expression of a psychological state.

Although Speech Act Theory has its limitations, it is still a useful approach to adopt when conducting a preliminary classification of the communicative functions of the email messages. Taking all of the above into consideration, I have integrated Rice's (1997) categorisation and Waldvogel's (2005) classification system to produce the following speech act functions which were used to code the email messages in my dataset:

- Requesting action: speech act where someone is asked to perform a task which involves a physical act.

- Requesting information: speech act where someone is asked to give information which involves a verbal act.

- Requesting opinion, advice or suggestion: this covers questions seeking opinion, advice or suggestion which involves a cognitive act.

- Providing information: this corresponds to Waldvogel's (2005) information giving or Searle's (1969) representatives. 
- Giving opinion, suggestion or advice: this corresponds to Waldvogel's (2005) invitations where this speech act appears to be of more benefit to the reader. In other words, the readers are given the option to do something if it is beneficial to them.

- Expressing thanks, apologising, congratulating: this category corresponds to Searle's (1969) expressives where it is used to convey the speaker's positive feelings and attitudes.

- Expressing complaint or reprimand: this category corresponds to Searle's (1969) expressives where it is used to convey the speaker's negative feelings and attitudes.

All speech acts where someone is asked to do something, to give information or to give advice are given a general label 'requests' rather than 'directives'. The term 'requests' has been adopted in this thesis for two reasons: firstly, my participants used this term when referring to this particular speech act; and secondly, they were comfortable using this term rather than the term 'directive'. I also divided the speech act 'requests' into three sub-categories to differentiate the types of requests: requests for actions, requests for information and requests for opinion, advice and suggestion. Requests of any kind, regardless of the linguistic form, are classified as face-threatening by Brown and Levinson (1987) and can violate the recipients' sociality rights and/or face sensitivities in certain situations (Spencer-Oatey \& Franklin, 2009). The classification of requests and their pragmatic force are discussed further in Chapter 7.

Positive messages considered non-face-threatening to the recipient, such as expressing thanks, congratulating, acknowledging, were separated from negative messages which were considered face-threatening such as reprimanding or complaining. The writer whose message functions as a complaint or reprimand can be interpreted as someone who "doesn't care about (or is indifferent to)" the recipient's feelings or wants (Brown \& Levinson, 1987, p. 66). In other words, the message has the potential for violating the face-sensitivities of the recipients (Spencer-Oatey \& Franklin, 2009). Workplace emails may perform one function, two functions or they may also serve several functions. In short, they may be multi-functional. In cases where an email has multi-functions, I took 
note of all the various functions but I identified one main communicative function for each email.

In my study, the aim was to keep the categorisation simple. Because it was not possible to determine the perlocutionary force of an email, the functions of emails were judged by the researcher's interpretation based on several cues: observation information; receiver's reply which provided some clues of what he/ she understood the message intent to be; and receiver's interpretation via interview (if available). In most cases the function of the emails was straightforward, i.e. the main function appeared to be clear from the context. Email 5.1 is an example where the main function seemed clear.

Email 5.2 (SS29) NZ1

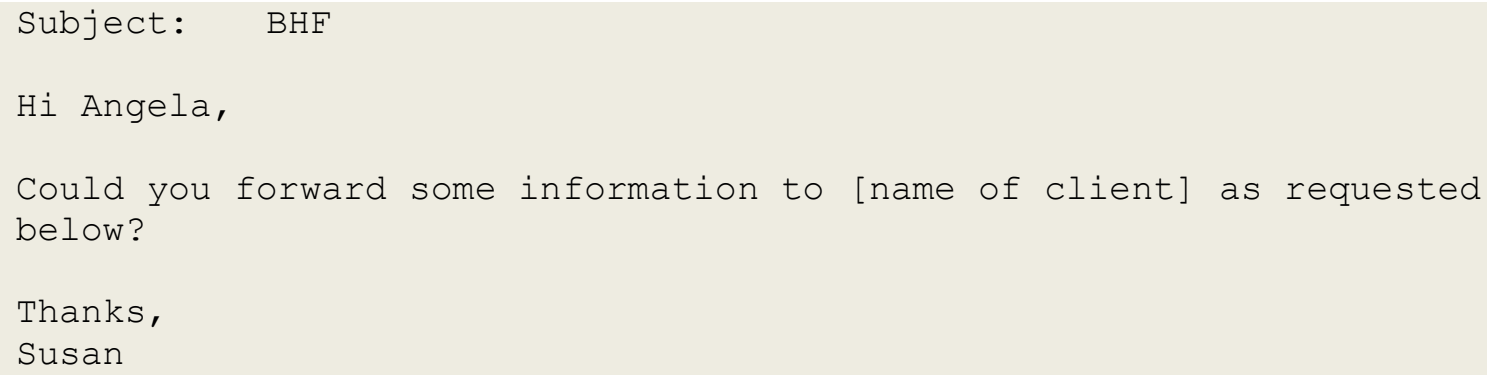

In Email 5.2, Susan requests Angela to email some information to the client 'Could you forward some information to [name of client] as requested below?' which is realised in the interrogative mood (Halliday \& Matthiessen, 2004). In this email, the message from the client is appended so that Angela is aware of the information that needs to be sent. This email was categorised as a request for action as it requires Angela to do a task, i.e. to email the client the information needed.

Although the main function in most emails was clear, it was not the case for some emails, and where there was more than one function or where the main function was not obvious, several steps were taken to address this problem. Firstly, the messages were read through to get a sense of the manner and context in which the email was written. Secondly, I adopted the role of a participant observer and relied on observation notes to understand the background of the messages. Textual, inter-textual clues and replies to emails (i.e. in some cases the perlocutionary effect) were also used to interpret the message. In some cases the participants were contacted (where possible) and they helped to fill in the background of the message which made an interpretation possible. 
To illustrate this problem, I discuss four emails. The first example is an email chain (Emails 5.3a and 5.3b) where the main function of the email was not clear. In this particular instance, the reply (Email 5.3b) provided contextual clues to ascertain the main function of the first message.

Email 5.3a (CM42a) NZ1

Subject: Bistro 107

This might be of interest to yo:

http://www. [website address]

Marcus

Email 5.3b (CS42b) NZ1

Subject: RE: Bistro 107

Thanks Marcus - might give it a go!

In the email chain (Emails 5.3a and 5.3b), it is uncertain whether the initial message (Email 5.3a) from Marcus was to give information of a potential sponsor or to make an implicit suggestion that Susan should contact this company. The main function seemed likely to be providing information if the recipient (Susan) did not act on it. However, based on the reply from Susan (Email 5.3b) who indicated that she would contact the company '... might give it a go!', the main function of this email was interpreted as giving suggestion. 
In the second example (Email 5.4), it was uncertain whether Megan's message directed to all staff members was to give information or make a request.

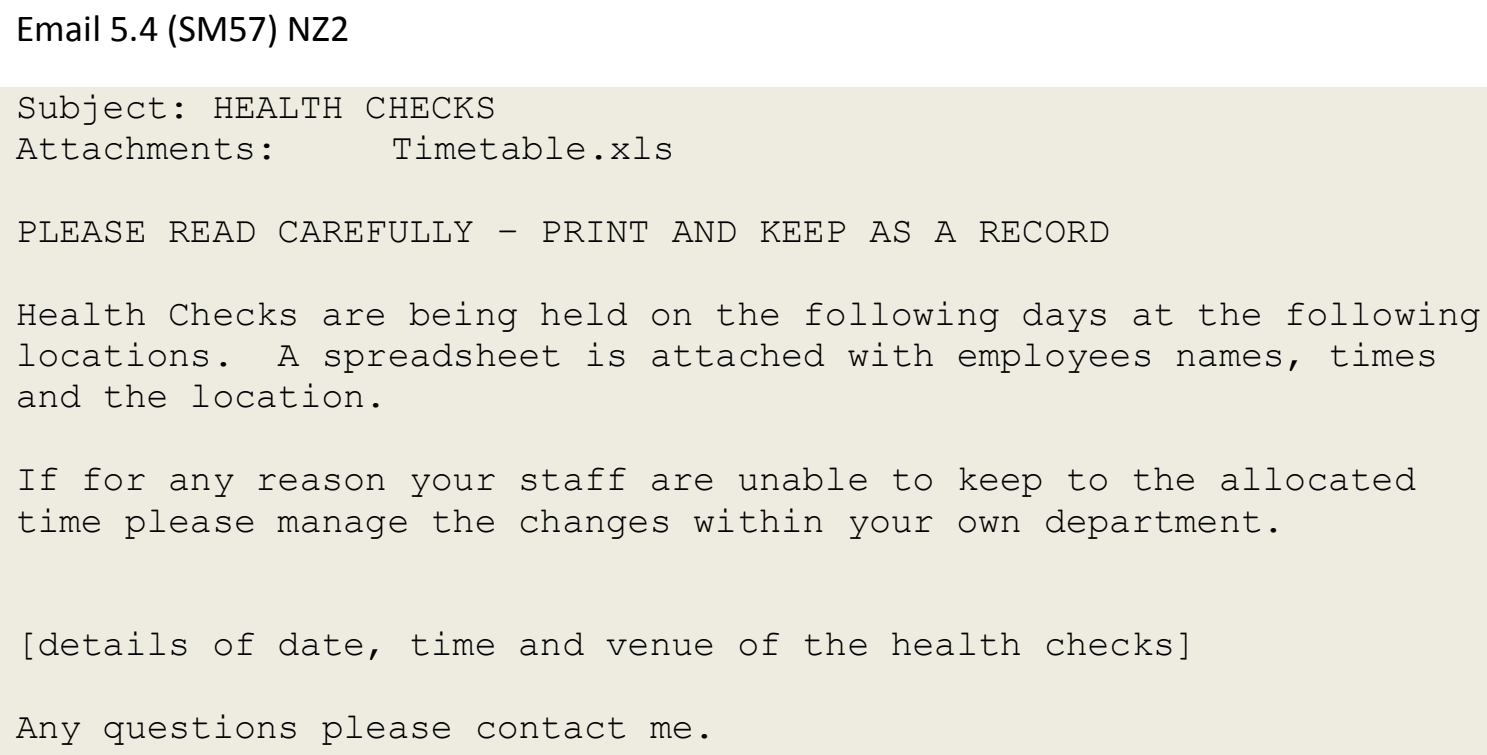

The main function of this email could be interpreted as providing information because a spreadsheet of employees' name and their health checks' schedule and location was appended in the email. This email could also be interpreted as a request that the staff members were to follow the schedule so that no employee missed out his/ her respective slot. In this particular instance, the observation notes collected proved to be a useful source when interpreting the main function of this email. In line with the company's values and emphasis on health and safety (see Section 4.4.5), this email was categorised as a request for action because in this company it is mandatory that all staff members have their annual health checks. In addition, Megan, the author of this email, states that employees have to manage the changes within their respective departments if they are not able to keep to the allocated slot. I also applied another strategy when ascertaining the main function of this email - inter-textual cues - the use of capital letters when making the request. The request, which is realised in the imperative mood accompanied by the request marker 'please', tells all staff members to do three things: read the email carefully; print the schedule and keep it as a reference; and attend the health check as scheduled. Hence, this email was classified as request for action (that all staff members adhere to the schedule prepared) rather than an information giving email though it clearly fulfilled that function as well. 
In the third example, where information was given as grounds for a request, the main function of the email was categorised as request. The use of a 'grounder' involves the speaker providing reasons, explanation or justification for the request (Blum-Kulka, Kasper, \& House, 1989b, p. 287). According to Brown and Levinson (1987), justification is used as a politeness strategy because in a workplace, justification is not necessary to gain cooperation (compliance) because the request is usually within the hearer's responsibilities. And, as explained above, requests could be asking someone to do something or asking someone to provide information or asking for advice. Email 5.5 is one example where information was given to make the request and to prepare the ground for it, the main function of the email was coded as a request.

Email 5.5 (SA49) NZ1

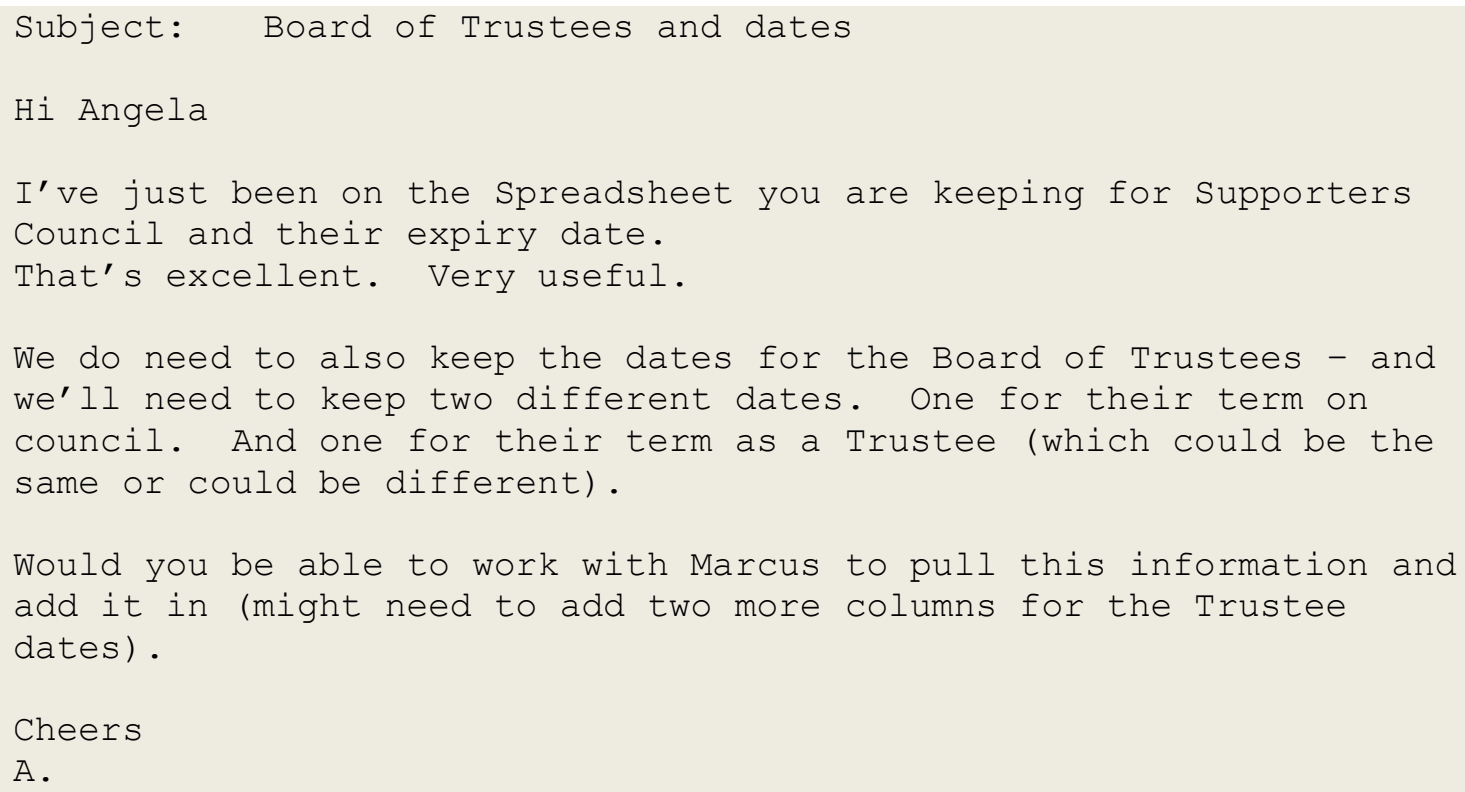

In Email 5.5, Andy requests Angela to work with Marcus to prepare an updated schedule to incorporate two columns for two types of dates for the members of Board of Trustees to keep them informed. The request is realised in the interrogative mood using the modal 'would' ('would you be able to work with Marcus ...'). The reasons provided in the second paragraph of the email prepare the ground for the request which explain why it is necessary to have two extra columns on the existing schedule that Angela had already prepared. In this instance, I classified the email's main function as a request for action. 
In the final example (Email 5.6), where there was uncertainty whether the email was to express apology or to provide information, the main function has been shown as expressing apology because the information given was leading up to why the apology was necessary.

Email $5.6($ SC20) M

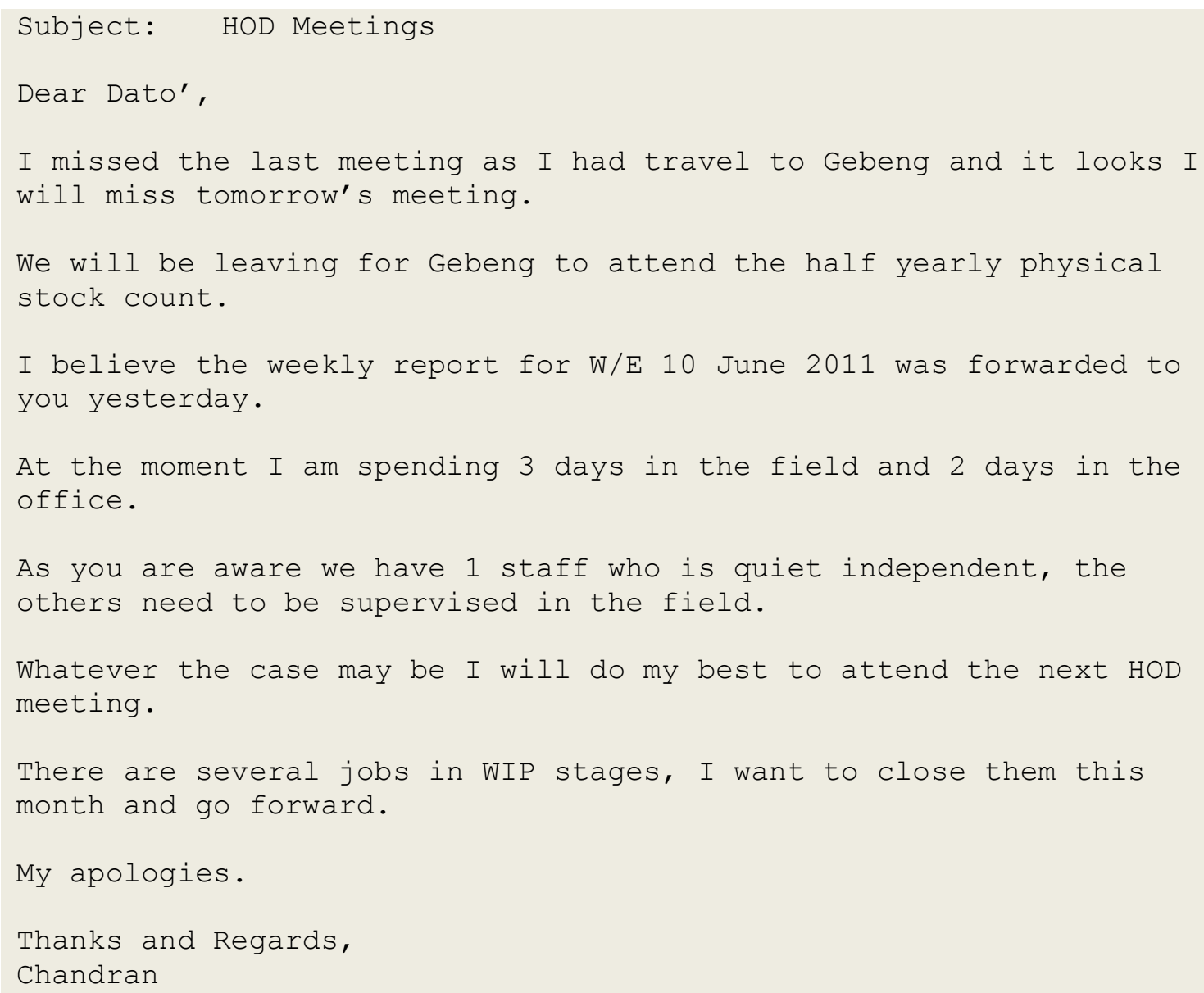

In this rather long email to his superior, the MD of the company, Chandran first greets him with the salutation 'dear' followed by the honorific epithet 'Dato'. He then informs the MD that he missed the previous meeting and that he will miss the next meeting (first paragraph). He also sends a courtesy copy of the email to his immediate superior, Amir, the CEO, to keep him informed of his (Chandran's) absence. In the next few paragraphs, he gives the reasons why he would miss the meeting. After the lengthy explanation, he gives assurance and promises that 'whatever the case may be I will do my best to attend the next HOD meeting', and then he returns to further explain ('there are several jobs in WIP stages') and justify ('I want to close them this month and go forward') why he would 
miss the subsequent meeting. This explanation could also be interpreted as preparing grounds for possibly missing the next meeting. Although his apology ('My apologies') comes at the end of the email, the major function of this email was categorised as expressing apology because the explanation provided prepared the ground for it.

The discussion above shows how the emails collected from the three workplaces were categorised into their main communicative functions. In the next section, a discussion of the main communicative functions of emails in each workplace is presented. This is divided into two parts: first, an overview of the main communicative functions of emails of the company is presented; then a discussion of workplace status and its influence on the type of communicative functions follows.

\subsection{Communicative functions of emails in three workplaces}

The three workplaces are dependent on email usage in their day to day business transactions. Although email provided the three workplaces a pathway for both internal and external communication, the usage of this technology differed greatly. In two workplaces (Company NZ2 and Company M), email was solely for internal use and business transactions, while in Company NZ1, there was a mixture of business and personal usage.

This section presents the results of the data analysis of the communicative functions of emails in the three workplaces, followed by a discussion of whether workplace status has an influence on the types of emails produced. 


\subsubsection{Company NZ1 emails}

Company NZ1's dataset comprises 611 emails from four key participants. Table 5.7 below shows the breakdown of the total emails by each key participant.

Table 5.7 Emails by key participants in Company NZ1

\begin{tabular}{lcc}
\hline \multicolumn{1}{c}{ Name of key participants } & Number of emails & Percentage (\%) \\
\hline Andy & 237 & 39 \\
Susan & 159 & 26 \\
Lesley & 128 & 21 \\
Rose & 87 & 14 \\
\hline Total & $\mathbf{6 1 1}$ & $\mathbf{1 0 0}$ \\
\hline
\end{tabular}

The dataset in Company NZ1 was made up of various proportions from the four key participants: Andy (39\%), Susan (26\%), Lesley (21\%) and Rose (14\%).

Abdullah (2003) claims that workplace status plays a role in accounting for the relative frequency of different communicative functions of the emails that were produced. The significance of workplace status is also recognised as an influencing factor in choosing the appropriate politeness strategies when conveying face-threatening acts such as making requests or expressing a complaint or reprimand (Brown \& Levinson, 1987; Scollon \& Wong Scollon, 2001). According to Abdullah's (2003) study of a multinational company, superiors tended to write more requests for action emails while subordinates reciprocated by providing information. Hence, there was a high number of emails which performed the function of requesting action in her (Abdullah) General Manager's dataset. In addition, she claims that subordinates were more likely to write emails requesting information than superiors. My findings, however, show different results as explained in the following paragraphs.

Chi-square tests were performed to test the significance of the influence of workplace status on three communicative functions - requesting action, requesting information and providing information - to verify if Abdullah's (2003) claims are true. Table 5.8 presents the main communicative functions for each key participant and the chi-square results. Then each of these functions is discussed in turn. 
Table 5.8 Main communicative functions of emails in Company NZ1 and Chi-square results

\begin{tabular}{|c|c|c|c|c|c|c|c|c|c|c|c|c|c|}
\hline \multirow[t]{3}{*}{ Communicative Functions } & \multicolumn{10}{|c|}{ Key Participants } & \multicolumn{3}{|c|}{ Chi-square test } \\
\hline & \multicolumn{2}{|c|}{ Andy } & \multicolumn{2}{|c|}{ Susan } & \multicolumn{2}{|c|}{ Lesley } & \multicolumn{2}{|c|}{ Rose } & \multicolumn{2}{|c|}{ Total } & \multirow[b]{2}{*}{$x^{2}$} & \multirow[b]{2}{*}{$d f$} & \multirow[b]{2}{*}{$p$ value } \\
\hline & No & $\%$ & No & $\%$ & No & $\%$ & No & $\%$ & No & $\%$ & & & \\
\hline Providing information & 84 & 35 & 86 & 54 & 48 & 38 & 55 & 63 & 273 & 45 & 28.641 & 3 & $.000 *$ \\
\hline Requesting action & 77 & 33 & 40 & 25 & 55 & 43 & 19 & 22 & 191 & 31 & 14.682 & 3 & $.002 *$ \\
\hline Expressing thanks, apologising, congratulating & 18 & 8 & 23 & 15 & 7 & 6 & 1 & 1 & 49 & 8 & 15.710 & 3 & $.001^{*}$ \\
\hline Requesting information & 33 & 14 & 2 & 1 & 4 & 3 & 4 & 5 & 43 & 7 & 29.085 & 3 & $.000^{*}$ \\
\hline Giving opinion, suggestion or advice & 20 & 8 & 5 & 3 & 10 & 8 & 1 & 1 & 36 & 6 & & $\mathrm{n} / \mathrm{a}$ & \\
\hline Requesting opinion, advice or suggestion & 4 & 2 & 3 & 2 & 3 & 2 & 6 & 7 & 16 & 3 & & $\mathrm{n} / \mathrm{a}$ & \\
\hline Expressing complaint or reprimand & 1 & $<1$ & 0 & 0 & 1 & $<1$ & 1 & 1 & 3 & $<1$ & & $\mathrm{n} / \mathrm{a}$ & \\
\hline Total & 237 & 100 & 159 & 100 & 128 & 100 & 87 & 100 & 611 & 100 & & & \\
\hline
\end{tabular}


Table 5.8 shows that staff members in Company NZ1 used emails mainly to perform two functions: providing information (45\%) and requesting actions (31\%). They used emails for other functions infrequently.

For the communicative function of requesting action, the chi-square test demonstrates that the participants differ significantly in how often they requested for action ( $\chi 2(d f=3)$ $=14.682, p=.002)$. In order to confirm whether superiors produced more emails requesting action than subordinates, pairwise comparisons were performed. These comparisons between the participants (Table 5.9), however, show that there is no significant difference in terms of writing emails requesting action between Andy, the CEO, and her subordinates. These findings suggest that Andy's status as a CEO does not seem to have influence on the communicative function of requesting action, which is surprising given the authority and legitimate power (French \& Raven, 1968; Thomas, 1995 ) vested in her. These findings also seem to support the company's characteristic of a relatively flat organisation structure. However, if the focus is on Lesley, the HOE, the findings tend to suggest that there was a subtle hierarchy observed. Under the original organisation structure, one Support Team member reported to one HOE, and Lesley seemed to adopt this old practice. Here, we see that Lesley (43\%) was more likely to request for action than Andy (33\%), a finding which contrasts with Abdullah's (2003) claim that superiors tended to write more emails requesting action. My observation field notes indicate that Lesley went on business trips more often than other HOEs. During her absences from the office, most of her emails performing the function of requesting action were directed to Rose who was supporting her event. This could contribute to an explanation of why she was more likely to request for action than Andy. 
Table 5.9 Results for Chi-square tests for the communicative function of requesting action

\begin{tabular}{lccc}
\hline Comparisons (raw data in brackets) & $\boldsymbol{\chi 2}$ & $\boldsymbol{d f}$ & $\boldsymbol{p}$ value \\
\hline Andy (77/237) vs. Lesley (55/128) & 3.954 & 1 & .047 \\
Andy (77/237) vs. Susan (40/159) & 2.458 & 1 & .117 \\
Andy (77/237) vs. Rose (19/87) & 3.462 & 1 & .063 \\
Lesley (55/128) vs. Susan (40/159) & 10.159 & 1 & $.001^{*}$ \\
Lesley (55/128) vs. Rose (19/87) & 10.245 & 1 & $.001^{*}$ \\
\hline
\end{tabular}

In order to avoid Type 1 errors, $p$-value was adjusted at $.01(.05 / 5 ; 5$ is the number of comparisons)

Regarding the function of requesting information, the overall result is statistically significant $(\chi 2(d f=3)=29.085, p=.000)$ (Table 5.8). Follow-up analysis (Table 5.10) that aims at understanding whether superiors write more emails requesting information than subordinates shows that there is some influence of workplace status on the production of this type of email between Andy and Susan, and Andy and Lesley but not between Andy and Rose. These results which suggest that Andy requested for information more often than her subordinates, Lesley and Susan, are in contrast with Abdullah's (2003) claim that subordinates tended to write more emails requesting information than superiors.

Table 5.10 Results for Chi-square tests for the communicative function of requesting information

\begin{tabular}{lccc}
\hline Comparisons (raw data in brackets) & $\mathbf{\chi 2}$ & $\boldsymbol{d f}$ & $\boldsymbol{p}$ value \\
\hline Andy (33/237) vs. Lesley (4/128) & 10.640 & 1 & $.001^{*}$ \\
Andy (33/237) vs. Susan (2/159) & 18.948 & 1 & $.000^{*}$ \\
Andy (33/237) vs. Rose (4/87) & 5.472 & 1 & .019 \\
Lesley (4/128) vs. Susan (2/159) & 1.208 & 1 & .272 \\
Lesley (4/128) vs. Rose (4/87) & .314 & 1 & .575
\end{tabular}

In order to avoid Type 1 errors, $p$-value was adjusted at .01 (.05/5; 5 is the number of comparisons)

The overall result of Chi-square test demonstrates that the difference between the participants for the function of providing information is statistically significant ( $\chi 2(d f=3)$ $=28.641, p=.000$ ) (Table 5.8). Follow-up analysis (Table 5.11) shows that both Susan, the Relationship Manager, and Rose, the Support Officer, used this function more frequently 
than Andy, a finding that supports other studies (Abdullah, 2003) where subordinates tended to write more emails providing information.

Table 5.11 Results for Chi-square tests for the communicative function of providing information

\begin{tabular}{lccc}
\hline Comparisons (raw data in brackets) & $\boldsymbol{\chi 2}$ & $\boldsymbol{d f}$ & $\boldsymbol{p}$ value \\
\hline Susan (86/159) vs. Andy (84/237) & 13.502 & 1 & $.000^{*}$ \\
Susan (86/159) vs. Lesley (48/128) & 7.839 & 1 & $.005^{*}$ \\
Susan (86/159) vs. Rose (55/87) & 1.916 & 1 & .166 \\
Rose (55/87) vs. Lesley (48/128) & 13.728 & 1 & $.000^{*}$ \\
Rose (55/87) vs. Andy (84/237) & 20.042 & 1 & $.000^{*}$
\end{tabular}

In order to avoid Type 1 errors, p-value was adjusted at $.01(.05 / 5 ; 5$ is the number of comparisons)

In sum, participants in Company NZ1 used emails mainly for providing information and requesting action; they used emails for other functions infrequently. My findings also suggest that workplace status appears to play a role in influencing some types of emails produced in Company NZ1. In the following sections, though, we see some different results in the other two workplaces.

\subsubsection{Company NZ2 emails}

Company NZ2's dataset comprises 575 emails from four key participants. Table 5.12 below shows the breakdown in raw counts and percentages of the total emails by each key participant.

Table 5.12 Emails by key participants in Company NZ2

\begin{tabular}{lcc}
\hline Name of key participants & Number of emails & Percentage \\
\hline Ken & 152 & 26 \\
Megan & 124 & 22 \\
Patrick & 156 & 27 \\
Sheila & 143 & 25 \\
\hline Total & $\mathbf{5 7 5}$ & $\mathbf{1 0 0}$ \\
\hline
\end{tabular}

In contrast with Company NZ1, the key participants in Company NZ2 contributed similar proportions of emails to each other: each contributed about one-quarter of the total email dataset. 
Table 5.13 shows that emails were used predominantly for providing information (48\%) and requesting action (28\%) in Company NZ2, a pattern that mirrors Company NZ1 (Section 5.4.1). Staff members used emails for other functions sporadically. As in Company NZ1, the four key participants in Company NZ2 used emails to provide information more often than other functions, and the second most frequent function was requesting action. Chi-square tests performed show that participants in Company NZ2 differ significantly in their use of two functions - requesting action $(\chi 2(d f=3)=9.509$, $p=.023)$ and providing information $(\chi 2(d f=3)=8.212, p=.042)-$ and these results are presented in Table 5.13. 
Table 5.13 Main communicative functions of emails in Company NZ2 and Chi-square results

\begin{tabular}{|c|c|c|c|c|c|c|c|c|c|c|c|c|c|}
\hline \multirow[t]{3}{*}{ Communicative Functions } & \multicolumn{10}{|c|}{ Key Participants } & \multicolumn{3}{|c|}{ Chi-square test } \\
\hline & \multicolumn{2}{|c|}{ Ken } & \multicolumn{2}{|c|}{ Megan } & \multicolumn{2}{|c|}{ Patrick } & \multicolumn{2}{|c|}{ Sheila } & \multicolumn{2}{|c|}{ Total } & \multirow[b]{2}{*}{$x 2$} & \multirow[b]{2}{*}{$d f$} & \multirow[b]{2}{*}{$p$ value } \\
\hline & No & $\%$ & No & $\%$ & No & $\%$ & No & $\%$ & No & $\%$ & & & \\
\hline Providing information & 87 & 57 & 54 & 44 & 66 & 42 & 68 & 48 & 275 & 48 & 8.212 & 3 & $.042^{*}$ \\
\hline Requesting action & 45 & 30 & 42 & 34 & 29 & 19 & 43 & 30 & 159 & 28 & 9.509 & 3 & $.023^{*}$ \\
\hline Requesting information & 5 & 3 & 7 & 6 & 14 & 9 & 11 & 8 & 37 & 6 & & $\mathrm{n} / \mathrm{a}$ & \\
\hline Expressing thanks, apologising, congratulating & 3 & 2 & 7 & 7 & 15 & 11 & 12 & 8 & 37 & 6 & & $\mathrm{n} / \mathrm{a}$ & \\
\hline Requesting opinion, advice or suggestion & 7 & 5 & 4 & 3 & 19 & 12 & 3 & 2 & 33 & 6 & & $\mathrm{n} / \mathrm{a}$ & \\
\hline Giving opinion, suggestion or advice & 5 & 3 & 7 & 6 & 12 & 8 & 6 & 4 & 30 & 5 & & $\mathrm{n} / \mathrm{a}$ & \\
\hline Expressing complaint or reprimand & 0 & 0 & 3 & $<1$ & 1 & $<1$ & 0 & 0 & 4 & $<1$ & & $\mathrm{n} / \mathrm{a}$ & \\
\hline Total & 152 & 100 & 124 & 100 & 156 & 100 & 143 & 100 & 575 & 100 & & & \\
\hline
\end{tabular}


Sherblom's (1988) study of a manager's in-box archive of 157 emails showed that the most common function of email was providing information. That means it is expected that managers in Company NZ2 write more emails performing this function. To examine whether Sherblom's (1988) finding is applicable in Company NZ2, I conducted a more detailed test (pairwise comparisons). The follow-up analysis (Table 5.14), however, shows that Megan, who was not a manager, used email to perform this function as frequently as the three managers. This suggests that workplace status does not seem to influence the type of emails produced by managers in Company NZ2.

Table 5.14 Results for the Chi-square test for the communicative function of providing information

\begin{tabular}{lccc}
\hline Comparisons (raw data in brackets) & $\boldsymbol{\chi} 2$ & $\boldsymbol{d f}$ & $\boldsymbol{p}$ value \\
\hline Megan (54/124) vs. Ken (87/152) & 5.121 & 1 & .024 \\
Megan (54/124) vs. Patrick (66/156) & .043 & 1 & .835 \\
Megan (54/124) vs. Sheila (68/143) & .429 & 1 & .512 \\
Ken (87/152) vs. Patrick (66/156) & 6.864 & 1 & $.009^{*}$
\end{tabular}

* In order to avoid Type 1 errors, p-value was adjusted at .0125 (.05/4; 4 is the number of comparisons)

In sum, the results above show two things: firstly, providing information and requesting actions dominated as the main communicative functions in each key participant's dataset in Company NZ2; and secondly, workplace status does not seem to influence the production of emails providing information. 


\subsubsection{Company M emails}

Company M's dataset comprises 559 emails from five key participants. In Table 5.15 below, the raw counts and percentages of the breakdown of the total emails by each key participant are presented.

Table 5.15 Emails by key participants in Company M

\begin{tabular}{lcc}
\hline Name of key participants & Number of emails & Percentage \\
\hline Amir & 157 & 28 \\
Chandran & 35 & 6 \\
Geetha & 109 & 20 \\
Sharifah & 133 & 24 \\
Kamal & 125 & 22 \\
\hline Total & $\mathbf{5 5 9}$ & $\mathbf{1 0 0}$ \\
\hline
\end{tabular}

Four key participants contributed between 22 to 28 percent of emails of the total dataset. The smallest set of emails was from Chandran, the HOD (6\%), while the highest contributor was Amir, the CEO (28\%). The relatively small number of emails donated by Chandran seems to support his claim (via interview) that he preferred face-to-face communication.

As discussed in Section 5.4.1, Abdullah (2003) claims that superiors were more likely to write emails requesting action and subordinates tended to respond by providing information. Chi-square tests were performed to test the significance of the influence of workplace status on the two most frequently used functions in Company $\mathrm{M}$ - requesting action (48\%) and providing information (38\%) - and these results are presented in Table 5.16. In the same table the results of the main communicative functions according to each key participant are also presented.

Supporting Abdullah's (2003) findings in the Malaysian context, in Company M, all the subordinates wrote more emails performing the function of providing information than the CEO in the dataset. A chi-square test performed shows a statistical difference between the participants for this function $(\chi 2(d f=4)=88.900, p=.000)$. 
Table 5.16 Main communicative functions of emails in Company $M$ and Chi-square results

\begin{tabular}{|c|c|c|c|c|c|c|c|c|c|c|c|c|c|c|c|}
\hline \multirow[t]{3}{*}{ Communicative Functions } & \multicolumn{12}{|c|}{ Key Participants } & \multirow{2}{*}{\multicolumn{3}{|c|}{ Chi-square test }} \\
\hline & \multicolumn{2}{|c|}{ Amir } & \multicolumn{2}{|c|}{ Chandran } & \multicolumn{2}{|c|}{ Geetha } & \multicolumn{2}{|c|}{ Sharifah } & \multicolumn{2}{|c|}{ Kamal } & \multicolumn{2}{|c|}{ Total } & & & \\
\hline & No & $\%$ & No & $\%$ & No & $\%$ & No & $\%$ & No & $\%$ & No & $\%$ & $x^{2}$ & $d f$ & $\begin{array}{c}p \\
\text { value }\end{array}$ \\
\hline Requesting action & 90 & 57 & 16 & 46 & 43 & 39 & 56 & 42 & 61 & 49 & 266 & 48 & 10.588 & 4 & $.032^{*}$ \\
\hline Providing information & 12 & 8 & 12 & 34 & 59 & 54 & 68 & 51 & 60 & 48 & 211 & 38 & 88.900 & 4 & $.000^{*}$ \\
\hline Requesting information & 38 & 24 & 0 & 0 & 2 & 2 & 2 & 2 & 0 & 0 & 42 & 8 & & $\mathrm{n} / \mathrm{a}$ & \\
\hline Giving opinion, suggestion or advice & 16 & 10 & 3 & 9 & 0 & 0 & 1 & $<1$ & 3 & 2 & 23 & 4 & & $\mathrm{n} / \mathrm{a}$ & \\
\hline $\begin{array}{l}\text { Expressing thanks, apologising, } \\
\text { congratulating }\end{array}$ & 0 & 0 & 3 & 11 & 2 & 5 & 2 & 2 & 1 & $<1$ & 8 & 1 & & $\mathrm{n} / \mathrm{a}$ & \\
\hline Expressing complaint or reprimand & 1 & $<1$ & 1 & $<1$ & 3 & $<1$ & 0 & 0 & 0 & 0 & 5 & $<1$ & & $\mathrm{n} / \mathrm{a}$ & \\
\hline $\begin{array}{l}\text { Requesting opinion, advice or } \\
\text { suggestion }\end{array}$ & 0 & 0 & 0 & 0 & 0 & 0 & 4 & 3 & 0 & 0 & 4 & $<1$ & & $\mathrm{n} / \mathrm{a}$ & \\
\hline Total & 157 & 100 & 35 & 100 & 109 & 100 & 133 & 100 & 125 & 100 & 559 & 100 & & & \\
\hline
\end{tabular}


For the communicative function of requesting action, the overall chi-square result shows a statistical difference between the participants $(\chi 2(d f=4)=10.588, p=.032)$. In order to confirm that there is a significant difference between the CEO and his subordinates in terms of requesting action, follow-up analysis (pairwise comparisons) was conducted. These comparisons (Table 5.17) show that Amir, the CEO (57\%), was more likely to use email to perform the function of requesting action than Sharifah, his subordinate (42\%), but when he was compared with Kamal, also his subordinate (49\%), Kamal wrote more emails performing this function than his CEO . One possible reason for this variation could be that it is a practice in this CofP that junior staff members must request their immediate HOD or the CEO to check the reports they wrote before sending these reports to external clients. This also suggests that both superior and subordinate used email to perform the function of requesting action to a similar extent.

Table 5.17 Results for Chi-square tests for the communicative function of requesting action

\begin{tabular}{lccc}
\hline Comparison (raw data in brackets) & $\mathbf{\chi 2}$ & $\boldsymbol{d f}$ & $\boldsymbol{p}$ value \\
\hline Amir (90/157) vs. Sharifah (56/133) & 6.672 & 1 & $.010^{*}$ \\
Amir (90/157) vs. Kamal (61/125) & 2.033 & 1 & .154
\end{tabular}

In order to avoid Type 1 errors, $p$-value was adjusted at .025 (.05/2; 2 is the number of comparisons)

Overall, participants in Company $\mathrm{M}$ used emails primarily to perform two functions: requesting action and providing information. The results also show that workplace status appears to have some influence on the production of emails performing some functions in Company M. For example, all the subordinates wrote more emails providing information than their CEO.

The following section presents a discussion comparing what the participants reported (from questionnaire and interview data) and what they actually did with reference to the email data. 


\subsection{Discussion: Three workplaces compared}

Email communication was used differently to achieve various communicative functions in these three workplaces. In this section, I first present a comparison of three communicative functions - providing information, requesting action and expressing thanks - in turn. This discussion is then compared to the findings from the questionnaires administered to the participants and interviews conducted in all the workplaces as discussed in Section 5.2.

In terms of communicative functions, the discussion above (Sections 5.4.1, 5.4.2 and 5.4.3) shows that there are slight differences between the three workplaces. Table 5.18 compares the distribution of emails according to the main communicative functions in the three workplaces. 
Table 5.18 A comparison of main communicative functions of emails in the three workplaces by percentage and Chi-square results

\begin{tabular}{|c|c|c|c|c|c|c|c|c|c|}
\hline \multirow[t]{3}{*}{ Communicative functions } & \multicolumn{6}{|c|}{ Companies } & \multicolumn{3}{|c|}{ Chi-square test } \\
\hline & \multicolumn{2}{|c|}{ Company NZ1 } & \multicolumn{2}{|c|}{ Company NZ2 } & \multicolumn{2}{|c|}{ Company M } & \multirow[b]{2}{*}{$x^{2}$} & \multirow[b]{2}{*}{$d f$} & \multirow[b]{2}{*}{$p$ value } \\
\hline & No & $\%$ & No & $\%$ & No & $\%$ & & & \\
\hline Providing information & 273 & 45 & 275 & 48 & 211 & 38 & 12.256 & 2 & $.002 *$ \\
\hline Requesting action & 191 & 31 & 159 & 28 & 266 & 48 & 56.029 & 2 & $.000^{*}$ \\
\hline Expressing thanks, apologising, congratulating & 49 & 8 & 37 & 6 & 8 & 1 & 26.711 & 2 & $.000^{*}$ \\
\hline Requesting information & 43 & 7 & 37 & 6 & 42 & 8 & & $\mathrm{n} / \mathrm{a}$ & \\
\hline Giving opinion, suggestion or advice & 36 & 6 & 30 & 5 & 23 & 4 & & $\mathrm{n} / \mathrm{a}$ & \\
\hline Requesting opinion, advice or suggestion & 16 & 3 & 33 & 6 & 4 & $<1$ & & $\mathrm{n} / \mathrm{a}$ & \\
\hline Expressing complaint or reprimand & 3 & $<1$ & 4 & $<1$ & 5 & $<1$ & & $\mathrm{n} / \mathrm{a}$ & \\
\hline Total & 611 & 100 & 575 & 100 & 559 & 100 & & $\mathrm{n} / \mathrm{a}$ & \\
\hline
\end{tabular}


Table 5.18 shows that in the two New Zealand workplaces, emails providing information dominated as the main communicative function accounting for almost half of the total number of emails: 45 percent in Company NZ1 and 48 percent in Company NZ2. This function ranks second in the Malaysian workplace (38\%). This finding concurs with Sherblom's (1988) US and Waldvogel's (2005) New Zealand research. In their study, emails performing the function of providing information were the primary function in the manager's dataset in Sherblom's (1988) study, and in the educational corpus in Waldvogel's (2005) research.

Chi-square tests were performed to examine whether there is a statistical difference in the use of emails performing three communicative functions - requesting action, providing information and expressing thanks - across the three workplaces. The results are presented in Table 5.18 and each of these functions is discussed in turn.

Chi-square result for the function providing information is statistically significant ( $\chi 2$ $(d f=2)=12.256, p=.002)$, suggesting that the difference in the number of this type of emails across the three workplaces is significant. Follow-up analysis (Table 5.19) that aims to examine which types of email functions were used most frequently reveals that both the New Zealand companies used emails to provide information more than Company M (38\%), and the two New Zealand workplaces did this at a similar rate $(45 \%$ and $48 \%)$.

Table 5.19 Results for Chi-square tests for the communicative function of providing information

\begin{tabular}{lccc}
\hline Comparisons (raw data in brackets) & $\mathbf{\chi 2}$ & $\boldsymbol{d f}$ & $\boldsymbol{p}$ value \\
\hline Company NZ1 (273/611) vs. Company NZ2 (275/575) & 1.179 & 1 & .278 \\
Company NZ1 (273/611) vs. Company M (211/559) & 5.788 & 1 & $.016^{*}$ \\
Company NZ2 (275/575) vs. Company M (211/559) & 11.760 & 1 & $.001^{*}$
\end{tabular}

In order to avoid Type 1 errors, $p$-value was adjusted at .017 (.05/3; 3 is the number of comparisons)

In contrast, the Malaysian data in my study exhibited a different distribution; emails requesting actions were the most frequent function for which email was used (48\%) when compared with the other two New Zealand workplaces (31\% and $28 \%$ ). The overall 
Chi-square result shows there is a significant difference between the three workplaces for this function $(\chi 2(d f=2)=56.029, p=.000)$ (see Table 5.18). Follow-up analysis (Table 5.20) confirms that the Malaysian workplace (Company M) used emails for requesting actions more than the two New Zealand workplaces. This finding supports the CEO's assertion (from the interview data) that making requests via email was a shared repertoire in this CofP and this practice was highly encouraged (see Section 4.5.2).

Table 5.20 Results for Chi-square tests for the communicative function of requesting action

\begin{tabular}{|l|c|c|c|}
\hline Comparisons (raw data in brackets) & $\boldsymbol{\chi 2}$ & $\boldsymbol{d f}$ & $\boldsymbol{p}$ value \\
\hline Company NZ1 (191/611) vs. Company NZ2 (159/575) & 1.854 & 1 & .173 \\
Company NZ1 (191/611) vs. Company M (266/559) & 32.683 & 1 & $.000^{*}$ \\
Company NZ2 (159/575) vs. Company M (266/559) & 48.061 & 1 & $.000^{*}$ \\
\hline
\end{tabular}

In order to avoid Type 1 errors, p-value was adjusted at .017 (.05/3; 3 is the number of comparisons)

Overall, while the two New Zealand workplaces were more likely to use emails to provide information than the Malaysian workplace, the latter used emails for requesting action more frequently than the former.

The actual email data for the two functions discussed above matched what the participants reported they do (see Tables 5.2, 5.4 and 5.6). Participants in all the three workplaces reported that they 'always' and 'often' use emails for providing information and requesting action. Therefore, it is no surprise that both these two functions stood out as the main communicative functions in all the three workplaces.

Generally people tended to report that they use emails to convey positive feelings and attitudes. For example, 61 percent of the participants surveyed in the Malaysian workplace reported that they 'always' and 'often' use email to say thank you (see Table 5.6). However, only one percent of the emails in the dataset performed this function. Chi-square test shows the workplaces differed in how often they expressed thanks $(\chi 2$ $(d f=2)=26.711, p=.000)$ (See Table 5.18). 
Further analysis (Table 5.21) confirms the observation that emails expressing thanks were less frequent in the Malaysian data than the two New Zealand datasets ( $8 \%$ and $6 \%$ respectively). This finding seems to challenge the widely practised Malaysian culture, particularly the Malay culture such as 'budi' (courteous) and 'hormat'(respect) in social interactions and warrants further investigation (Abdullah, 1996; see Paramasivam, 2007 for details on 'budi' and 'hormat'; see Ramli, 2013 for explanation on 'budi'). This result suggests that people typically overestimate their use of emails for conveying positive feelings.

Table 5.21 Results for Chi-square tests for the communicative function of expressing thanks

\begin{tabular}{lccc}
\hline Comparisons (raw data in brackets) & $\chi 2$ & $d f$ & $p$ value \\
\hline Company NZ1 (49/611) vs. Company NZ2 (37/575) & 1.106 & 1 & .293 \\
Company NZ1 (49/611) vs. Company M (8/559) & 27.343 & 1 & $.000^{*}$ \\
Company NZ2 (37/575) vs. Company M (8/559) & 18.622 & 1 & $.000^{*}$ \\
\hline
\end{tabular}

In order to avoid Type 1 errors, $p$-value was adjusted at $.017(.05 / 3 ; 3$ is the number of comparisons)

To summarise, in the two New Zealand companies, participants reported that they use email to provide information more than other functions, and this is supported by the actual email data. In the Malaysian workplace, when email was used as a mode of communication, the actual email data shows that the most frequent function used is requesting action and this appears to be at odds with what the participants reported because only 23 percent of the Malaysian participants reported that they 'always' and 'often' use emails for making requests. From the discussion above, the findings that email was used mainly for two functions - providing information and making requests support the results found in other research (Abdullah, 2003; Kankaanranta, 2005; Sherblom, 1988; Waldvogel, 2005).

\subsection{Conclusion}

These three case studies confirmed that email has become an important communication mode in the workplaces. At Company NZ1, although the physical layout of the 
organisation facilitated face-to-face interactions, email communication was fully embraced by the staff members. Close analysis shows that the use of email communication increased when the CEO and HOEs travelled to other parts of the country. In contrast, at Company NZ2, email communication was influenced by the physical dispersal of the organisation. Because it was a large international organisation and operated in different parts of New Zealand, face-to-face communication was not always a practical option, hence email was one feasible choice. In addition, the nature of the industry - one which required written documentation - had an impact on the high usage of email communication. The communication choice in Company $\mathrm{M}$, on the other hand, was largely determined by the workplace culture. As reported in Section 5.2.3, the CEO strongly encouraged the use of email and the high usage of emails in this workplace was reflected in the actual email dataset. Although email affected the participants' working day, none of them reported that the technology is a waste of time.

This chapter has explored the different communicative functions in three workplaces, demonstrating that email was used for the same main functions but the proportions of usage were different. Although the three workplaces used emails to perform various communicative functions, two functions stood out: providing information and requesting action.

The discussion in this chapter also shows that the workplace status of the key participants in the two New Zealand workplaces did not seem to play a role in determining the functions of emails that were written. However, in the Malaysian data, subordinates tended to write more emails performing the function of providing information, a finding that mirrors that of Abdullah's (2003) study.

The comparison between what the participants reported and the actual emails shows two things. First, there is agreement that email was used for providing information: emails performing this function dominated the datasets in the two New Zealand workplaces and stood at second place in the Malaysian workplace. Second, there is an inconsistency between what people say they do and what they actually do for some communicative functions. For example, in Company $M$, although 61 percent of the participants reported that they 'always' and 'often' use email to say thank you, only one percent of the total emails performed this function. 
As stated in section 5.3, emails can perform one function, two functions or they can be multifunctional. Besides performing transactional functions, emails can also build interpersonal relationships. In other words, emails can accomplish social and affective functions by means of greetings and closings and other linguistic features such as mitigating strategies, process types (relate to the ideational metafunctions) and mood choices (relate to the interpersonal metafunctions). In Chapters 6 and 7, I present a thorough discussion of greetings and closings and the different types of linguistic features respectively. 


\section{Chapter 6: How Greetings and Closings Contribute to Power Relations and Solidarity}

\subsection{Introduction}

In this chapter I explore how relational meaning and power are constructed within the CofP (see Chapter 4 for a full discussion of CofP) in the three workplaces by focussing on the use of greetings and closings and how they do power and collegiality. An investigation of the presence and absence of greetings and closings can provide insights into people's interpersonal interactions. Analysis of the types of greeting and closing can also indicate people's relational practices at their workplaces. In this chapter, the literature on greetings and closings, focussing particularly on emails, is discussed first. Then I present a discussion of the use of greetings and closings in the three workplaces.

\subsection{Greetings and closings: An overview}

Eckert and McConnell (2003, p. 138) state that "greetings and farewells offer formulas to ease the strain created for face by the beginnings and end of interactions." This practice is evident in all kinds of spoken communication. For instance, Schegloff (1986) suggests that a telephone conversation begins with a series of ringing tones which summon the answerer to pick up the phone. The answerer typically initiates the beginning of the conversation by uttering a simple 'hello'. According to Laver (1975, p. 218) "the function of the behavioural role that characterises the opening phase is to lubricate the transition from non-interaction to interaction, and to ease the potentially awkward moments of the encounter ... before the main business of the encounter is embarked upon in the medial phase. The closing phase is once again a transitional phase, easing the transition from full interaction to departure". These practices have been extended to letter writing as well as to email communication. Previous studies on emails show that many emails begin with some form of greeting and end with a closing/ sign off (Abdullah, 2006; Alsree, 1997; Gains, 1999; Ho, 2009; Kankaanranta, 2005; Nickerson, 1999; Waldvogel, 2005).

In emails, greetings and closings may be used as a strategy to personalise messages and this can be seen as an attempt of doing solidarity as email writers are able to use different types of greeting and closing expressions to signal closeness and distance. In other words, 
greetings and closings act as a means of reinforcing status relationships. According to Waldvogel (2005) who explores the relationship between workplace culture and the role, status and style of email in her study, greetings and closings are considered important because they introduce a personal touch into the email message and help build working relationships in an organisation. She also says that the type of greeting and sign off signals social distance or solidarity between staff members in the organisation.

Kankaanranta (2005), in her study of email messages written in the lingua franca English by Finns and Swedes in a multinational corporation observes that salutations (greetings), closings and signatures contribute to social relations because they create a personal tone for the messages. She notes that the use of a salutation followed by first name in 80 percent of her emails was more frequent among non-native English speakers compared to native speakers and offers two reasons for this. The first reason is that because email originated from the American internal memo that contains no salutation, the American writers carried over this style into emails but the Swedish and Finnish writers did not. The second reason is that the writer "constructs a relationship with the recipient, and the usage thus contributes to the maintenance of good social relations" with the presence of salutations (Kankaanranta, 2005, p. 359). I find the researcher's second reason problematic because native speakers also use salutations for the same reason - to build rapport with their recipients. Similarly, her study also suggests that the use of signatures and closings also gives a positive effect in building social relations.

In Ho's (2009) study on request emails by a team of English lecturers in a Hong Kong public education institution, the leaders in this team used greetings and closings as a mechanism to build rapport with their subordinates. By addressing the recipient(s) directly, the leaders considered the interpersonal element. In addition, the use of greetings reduced the distance between themselves (the leaders) and their subordinates (the recipients) by getting them (the subordinates) involved. And by including a closing element, the leaders were seen as doing collegiality with their subordinates.

In the next section, I present a discussion of the roles of greetings and closing expressions and then I discuss their usage in the three workplaces. 


\subsubsection{Roles of greetings}

Greetings perform various functions. First, greetings are ways in which writers construct their relationship as well as their social and professional identities with their audience. Second, greetings "open communicative acts and set the tone for the interexchanges that follow" (Wood \& Kroger, 1991, p. 145). Third, greetings often give information about the writer's attitude, personality, status, age and gender (Waldvogel, 2005).

Greeting can take various forms. Greeting in English can involve a salutation of some sort and a term of address (or form of address). A salutation can be formal (i.e. 'Dear') or informal (i.e. 'Hi', 'Hey', 'Hello'). Similarly a term of address can also be formal (i.e. title followed by last name or honorific epithets) or informal (i.e. first name only). In emails, writers may or may not include a greeting. And if a greeting is present, it may or may not include a salutation and it may or may not include a term of address. Greeting can take one of the following formats:

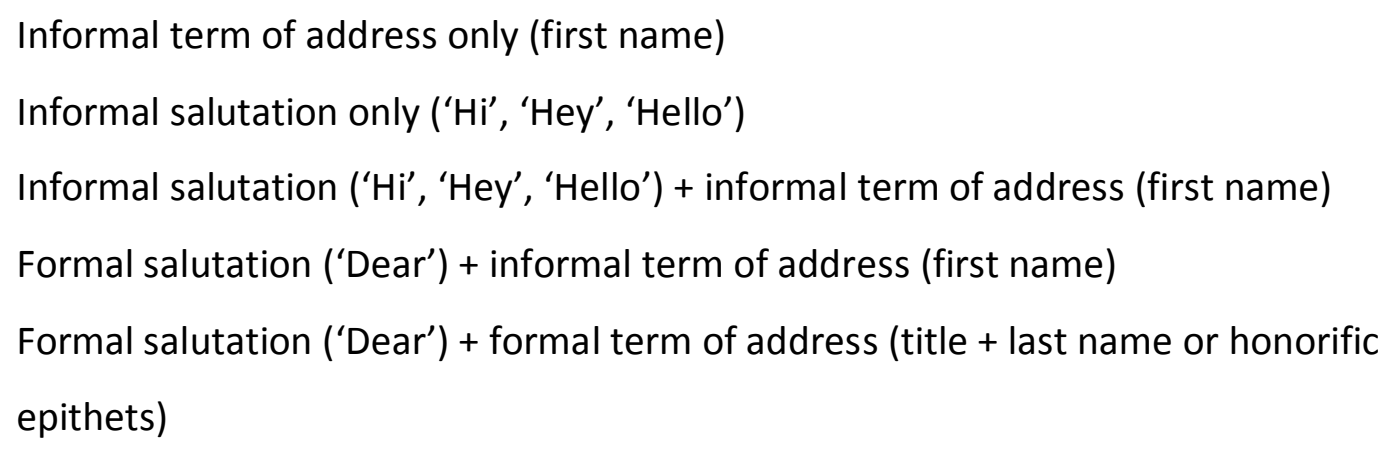

Morand (1996, p. 423) defines forms of address as "how individuals name each other, for instance, by title/ last name (Ms. Smith) or first name (Jim)". He explains that "while such forms [terms of address] comprise only brief moments of interaction, they are nevertheless critical events that are key to defining ensuing role orientations. Address forms signal and demarcate what types of emotional and interpersonal access two actors are to enjoy towards each other" (Morand, 1996, p. 423). In addition, Wood and Kroger (1991) claim that address forms "establish, at least initially, the relative power and distance of speaker and hearer".

Based on Morand's (1996) definition, terms of address are an aspect of politeness. In Brown and Levinson's (1987) politeness theory, more formal terms of address indicate negative 
politeness, while more informal forms of address suggest solidarity relations with the recipient. Laver (1975, p. 224) claims that the use of different categories of opening (greeting) is normally determined by the status of the two speakers involved in the interaction. In other words, status influences the greetings used. He suggests three levels of interaction: upward, downward and equal (Laver, 1975, p. 223). In an interaction between equals where a solidary relationship has been established, both interactants are free to choose the opening remark. In upward interactions, the subordinate may choose a formal greeting unless solidarity already existed. In contrast, in downward interactions, the superior can choose a greeting which signals formality or solidarity.

Alsree (1997, p. 217) argues that "naming conventions are not only context-bound, they are also culture-bound". In a typical English-speaking culture, the use of first name signals a positive politeness strategy while a more formal greeting, such as 'title + last name' indicates a negative politeness strategy which signals both power and social distance. A formal greeting such as 'title + last name' is usually used asymmetrically by a subordinate to a superior while the superior uses first name to address his/ her subordinates. In contrast, when formal greeting such as 'title + last name' or 'title + first name' is used in a symmetrical relationship, it indicates that the social distance and power is greater between the two interactants (Morand, 1996).

Although Malaysia is described as having a high regard for power hierarchy, Abdullah (1996) notes that the country practices a high context culture ${ }^{17}$ where it is important to engage in social pleasantries, and one way of establishing rapport is through the use of greetings and closings. But because the culture places great emphasis on titles and forms of address, the use of first name alone seldom occurs unless the recipient is a close associate of the writer or if they are at the same level of status. The use of first name alone signals either "a degree of familiarity or a breakdown of traditional norms"(Abdullah, 2003, p. 317). The address forms used are complex due to the different rules for each ethnicity and exceptions are made for recipients conferred with special titles, in which case honorific epithets such as 'Dato', 'Yang Berhormat' (the Right Honourable) or 'Yang Berbahagia' (the Honoured One) are used to address them. And as Alsree (1997, p. 148) notes in her study, the longer the

\footnotetext{
${ }^{17}$ According to Abdullah (1996), people in high context culture tend to view social pleasantries as an important criterion to establish a personal relationship.
} 
greeting form, the more formal it is, hence signaling greater social distance between interactants. Table 6.1 which is drawn from Abdullah (2003, p. 314) shows the different greeting forms used in Malaysia.

Table 6.1 Patterns of address forms in Malaysian society (Abdullah, 2003, p. 314)

\begin{tabular}{|c|c|c|c|c|c|}
\hline Ethnicity & First name & $\begin{array}{l}\text { Middle } \\
\text { name * }\end{array}$ & $\begin{array}{l}\text { Last/ family } \\
\text { name }\end{array}$ & Formal written $* *$ & $\begin{array}{l}\text { Informal } \\
\text { written }\end{array}$ \\
\hline $\begin{array}{l}\text { Anglo } \\
\text { American }\end{array}$ & Michael & James & Taylor & Mr Taylor & Michael \\
\hline Malay & Mohd Azlan & bin (son of) & Akbar Shah & Encik Mohd Azlan & Mohd or Azlan \\
\hline \multirow[t]{2}{*}{ Chinese } & Swee & Lee & Wong & $\begin{array}{l}\text { Miss/ Cik Wong } \\
\text { Or }\end{array}$ & $\begin{array}{l}\text { Wong } \\
\text { Or }\end{array}$ \\
\hline & Susan & S.L. & Wong & $\begin{array}{l}\text { Miss/ Cik Wong } \\
\text { Swee Lee or Miss/ } \\
\text { Cik Susan }\end{array}$ & $\begin{array}{l}\text { Swee Lee or } \\
\text { Susan }\end{array}$ \\
\hline Indian & $\begin{array}{l}\text { Ramasamy } \\
\text { David }\end{array}$ & $\begin{array}{l}\text { s/o (son of) } \\
\text { N. }\end{array}$ & $\begin{array}{l}\text { Navendran } \\
\text { Ramasamy }\end{array}$ & $\begin{array}{l}\text { Mr/ Encik } \\
\text { Ramasamy Or } \\
\text { Mr/ Encik David }\end{array}$ & $\begin{array}{l}\text { Ramasamy Or } \\
\text { Rama Or Samy } \\
\text { Or David }\end{array}$ \\
\hline Eurasian & Peter & Augustin & Monteiro & $\begin{array}{l}\text { Mr/ Encik } \\
\text { Monteiro } \\
\text { Mr/Encik Peter }\end{array}$ & Peter \\
\hline
\end{tabular}

*In Malay, Chinese or Indian communities these may not be names, but markers (e.g. 'bin', 'son of' or 'S.L.' initials standing for Chinese given names, or ' $N$ ' initial standing for father's name) that occur in a median position between first name and last name.

** Malay titles ('Encik' [Mr], 'Cik' [Miss], 'Puan' [Mrs]) are widely used in Malaysia instead of the Anglo American equivalents.

Alsree (1997, p. 145) suggests that while formal address forms can be determined by gender (Mr, Mrs, Ms and Miss), they can also be influenced by other factors such as "age (Master), relationship (uncle, grandfather), marital status (Mrs), [...] religion (Bishop, Cardinal), status (Lord, His Excellency, Her Highness), profession (Lieutenant, Captain) and expertise (Professor, Doctor)". Informal address terms such as terms of endearment or nicknames that are only used between close friends or colleagues are usually in-group identity markers (Wenger, 1998a). In written communication, the convention for formal letters written in English is to include the word 'Dear' followed by the recipient's title and last name (e.g. 'Dear Mr. Y') which is also evident in email messages. However, if the writer is an acquaintance of the recipient, the writer may dispense with the title and use only the first name instead of the last name (e.g. 'Dear John').

In the context of politeness theory, the choice of greeting often suggests the degree of distance or relationship between interactants. Levinson $(1983$, p. 63) describes the choice of 
greeting as a "social deixis (since they encode) social distinctions that are relative to particular roles, particular aspects of the social relationships holding between speaker and hearer and some referent". In email communication, it is the writer, the person who initiates the message, who sets the tone of the email exchange as well as the social relationship of the interaction through the linguistic choices s/he makes. In responding, the recipient can then choose to reciprocate the message by using the same type of greeting or to challenge the set tone.

Studies of email in different countries show that there is no a standard type of greeting used in business emails. Gimenez (2000, p. 245) who studied 63 emails from an import-export company based in the UK notes that a range of greetings used in emails suggests a "more relaxed style when compared with the more rigid one in formal commercial letters". He finds that the writers' use of greetings range from no salutation to the more conventional 'dear + first name' or 'dear + title + last name'. In a study conducted in New Zealand, Waldvogel (2007) finds that the most common informal types of greetings are 'hi + first name' or first name only. She argues that the formal greeting 'dear + first name' shows formality or deference. She also claims that the absence or presence of any greeting could be intentional, i.e. to signal solidarity or deference, or unintentional, i.e. a linguistic habit.

In research conducted in the US, Rice (1997, p. 18) finds 59 instances (30\%) of "personal greetings" in the sample of 200 email business memos he analysed. Nickerson (1999) who studied 100 emails from a large multinational corporation in the Netherlands notes that salutations were optional in email messages and were included only if the message was intended for a primary (single) recipient. Gains (1999) examined 116 randomly selected emails in a commercial and an academic (university) context in the UK. He finds that 92 percent of the email exchanges in the commercial context did not include any greeting and 63 percent of the emails in the academic context began with some form of greeting.

In contrast, in Li's (2000b) study of non-native English speakers in Hong Kong, 95 percent of emails contained a greeting. In the Malaysian context, Abdullah (2003) and Alsree (1997) find that writers used a range of opening greetings which signal sensitivity to the status of the recipients and to the weight of imposition (Brown \& Levinson, 1987). Alsree (1997) finds 114 occurrences of different address forms in her corpus of 141 email messages while 
Abdullah's (2003) study shows that more than 60 percent of all email messages had one salutation or combined salutations. Ho (2009), who studied 115 emails in a public academic institution in Hong Kong, observes that greetings were used more frequently in downward request emails.

\subsubsection{Roles of closings}

While greetings set the tone of an email, closings (sign off, farewell) have two purposes. First, closings signal some form of closure which can be seen as an attempt to end an email on a positive note or "establish a continuing consensus of future interactions" (Waldvogel, 2005, p. 149). Crystal (2006) claims that the farewell element performs two functions: boundary marker and extended identity function. He explains that as a boundary marker, it signals that "further scrolling down is unnecessary" (p. 110) and it presents "fuller identification" of the writer, especially in forwarded emails or emails with attachments.

Second, a closing expression is seen as a final word that writers use to re-establish an interpersonal relationship with their recipient(s) besides signalling some form of closure. Contrary to the more prescriptive complimentary close which is found in formal letter writing (see Searle, 2003), closing expressions in emails are more flexible and less rigid. Closing expressions display fewer variations. The closing expressions found in emails are not the standard expressions such as 'yours sincerely' or 'yours faithfully' which are commonly found and typical in formal business letters (Searle, 2003).

The closing expression 'cheers' is considered less formal and more friendly than 'regards' or 'kind regards' and this expression is generally used among colleagues in an organisation. In emails, a closing can take the following format:

- a closing expression only

- first name only

- signature block only

- a closing expression + first name

- a closing expression + first name + signature block

The importance of closing expressions is viewed differently by some researchers. Sherblom (1988, p. 44) regards closing expressions as "informationally redundant" because signing the 
email "did not add any additional information about the identity of the sender". None of his emails sent down the hierarchy were signed off. This was due to the feature of the email format where the sender's name was generated automatically. In agreement with Sherblom (1988), in 2013, a New York writer, Matthew Malady, sparks a fiery debate after he suggested putting an end to the written sign off in email which he labelled as "awkward" and "a waste of time" when it comes to online communication. He claims that people should end an email with the "actual last thing" that they wanted to say (Davies, 2013). In contrast, Ho (2009) observes that the leaders in a team of education professionals in Hong Kong included a sign off to signal solidarity. He argues that a sign off was included as a rapport building mechanism between the leaders and their subordinates in the public education institution where he conducted his study. Supporting Ho (2009), Waldvogel (1999) finds that nearly all women (97\%) and a majority of men (87\%) who sent their messages upward, and five out of six messages sent downward included a sign off in her sample of 49 emails. She attributes this result to the egalitarian New Zealand attitude and collegial atmosphere displayed in the workplace.

Another feature which can be present at the end of an email is a signature block. A signature block is usually automatically generated by the email software and it precedes a closing expression. According to Baym (1998), it is one of the ways of creating one's identity. She argues that a signature block is used as a means of management identification through the use of quotations, graphics, and/ or philosophical slogans (Baym, 1995). While most signature blocks consist of information about the writer such as the company logo, the writer's name, organisational designation, postal address, telephone contact number(s), fax number and/ or website address, some are pre-programmed to include a closing expression. In my study, in all the three workplaces, the signature block did not include a closing expression and it was usually appended consciously when the writer corresponded with an external client. An example of a signature block, which displayed almost the same information in all the three workplaces, is:

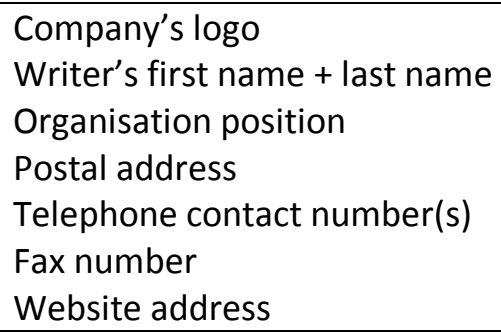


In sum, while the presence and absence and types of greetings and closings used could be a linguistic habit as argued by Waldvogel $(2005,2007)$, it could also perform various functions: (1) the writer's attempt at building rapport; (2) the writer's way of signalling distance with his/ her audience; and (3) the writer's strategy of reinforcing status relationships.

\subsection{Use of greetings and closings in Company NZ1}

In this section, first, I present frequencies in the use of greetings and closings in emails in Company NZ1. Then I discuss the use of greeting and the types of greetings used by the participants in this workplace. Lastly, I present a discussion of the use of closing and the types of closings found in the dataset. I will follow the same sequence to present the analysis for Company NZ2 and Company M in sections 6.4 and 6.5 respectively below.

As stated in Section 3.5.4, a total of 611 email messages were collected from Company NZ1. Table 6.2 shows frequencies in the use of greetings and closings in emails.

Table 6.2 The use of greetings and closings in emails in Company NZ1

\begin{tabular}{|c|c|c|c|c|c|c|c|c|c|}
\hline \multirow[t]{2}{*}{$\begin{array}{l}\text { Greeting } \\
\text { and } \\
\text { Closing }\end{array}$} & \multicolumn{2}{|c|}{$\begin{array}{l}\text { New or Primary } \\
\text { Message }\end{array}$} & \multicolumn{2}{|c|}{$\begin{array}{c}\text { Chain emails } \\
\text { (follow-up } \\
\text { messages) }\end{array}$} & \multicolumn{2}{|c|}{ Total } & \multicolumn{3}{|c|}{ Chi-square test } \\
\hline & No & $\%$ & No & $\%$ & No & $\%$ & $x^{2}$ & $d f$ & $\begin{array}{c}p \\
\text { value }\end{array}$ \\
\hline No greeting & 128 & 38 & 153 & 56 & 281 & 46 & & & \\
\hline Greeting & 210 & 62 & 120 & 44 & 330 & 54 & 20.082 & 1 & $.000^{*}$ \\
\hline No closing & 141 & 42 & 112 & 41 & 253 & 41 & & & \\
\hline Closing & 197 & 58 & 161 & 59 & 358 & 59 & 0.030 & 1 & .863 \\
\hline Total emails & 338 & 100 & 273 & 100 & 611 & 100 & & & \\
\hline
\end{tabular}

In the Company NZ1 dataset, Table 6.2 shows that staff members used greetings more often in new or primary emails (62\%) than in chain emails (44\%). However, they signed off at similar frequencies in both kinds of email ( $58 \%$ and $59 \%$ respectively). Chi-square tests show there is a significant difference in the occurrence of greeting in new and chain emails in this workplace $\left(\chi^{2}(d f=1)=20.082, p=.000\right)$, but the use of a closing expression in new and chain emails does not differ statistically $(\chi 2(d f=1)=0.030 p=.863)$. Overall, these results suggest that staff members in this CofP aimed to construct positive relationships with each other through the use of greeting in new emails. These results could also suggest that greeting could be dispensed with when the messages became a conversation thread, a finding which 
concurs with Crystal's (2006, p. 105) observation that between people who know each other "greetingless messages are usually promptly sent responses, where the responder sees the message as the second part of a two-part interaction (an adjacency pair), for which an introductory greeting is inappropriate".

From the questionnaire findings it shows that most people liked to receive a message which started with some form of greeting. Ninety-three percent of those surveyed agreed or strongly agreed that it is important to start a message with a greeting of some sort. It can be a friendly ' $\mathrm{Hi}$ ' or the name of the recipient. The staff members also believed that it is important to use a greeting even when they are writing to close colleagues (87\%). Most people interviewed felt that it is professional and courteous to begin an email with a form of greeting. The common greeting used in this workplace was 'Hi' which was seen as informal. 'Dear' which is perceived as a formal greeting was usually used when corresponding with clients, for example 'Dear Clients'.

Table 6.3 shows the types of greeting used in emails in Company NZ1. The table shows that there was no consistency in the pattern of greeting used in the emails. Sometimes participants used greetings, and sometimes they didn't. And when they used greetings, the types of greeting used varied.

Table 6.3 Types of greeting used in emails in Company NZ1

\begin{tabular}{lcc}
\hline Greeting types & Number of emails & Percentage (\%) \\
\hline No Greeting & 281 & 46 \\
Greeting word only & 44 & 7 \\
First name only & 27 & 4 \\
Greeting word + first name & 242 & 40 \\
Thanks + Name & 17 & 3 \\
\hline Total & 611 & $\mathbf{1 0 0}$ \\
\hline
\end{tabular}

Table 6.3 shows that the most popular greeting used was the informal form ' $\mathrm{Hi}+$ first name' (40\%). When only a greeting word is used, the most common one was ' $\mathrm{Hi}$ ' (7\%). The greeting word 'Hey' followed by the recipient's name occurred only twice. When addressing several recipients in an email, the common and popular greeting used was 'Hi All' or 'Hi guys'. 
McConnell-Ginet $(2003$, p. 84$)$ states that the plural 'guys' is widely used when addressing a group "no matter what the composition of the group", which probably explains why the CEO used the familiarizer 'guys' when addressing her staff members who were mostly female. The formal greetings 'Good morning/ afternoon All' were used thrice to address all the staff members, and only one message in the dataset began with the Maori greeting 'Kia Ora'. Four percent of the messages started with just the recipient's name while only three percent of messages began with 'Thanks' followed by the name of the recipient(s). Closer analysis reveals that these messages were follow-up responses to the first message. This finding concurs with Waldvogel's (2005) analysis. She finds that 'Thanks' was used as an expression of gratitude for something done. Similarly, in my data, 'thanks' was used to express appreciation to the recipient for work done.

Table 6.3 also shows that 46 percent of the email messages contained no greeting. Email messages without greeting were either a quick reply or follow-up message, a forwarded message, a brief note or a message made to the appended attachment. Email 6.1 is one example of a quick reply where there was no greeting but the writer launched straight into business.

Email 6.1 (SR43) NZ1

Subject: RE: short term deposits [message]

In Email 6.1, Andy does not greet Marcus nor end the email with a closing expression. Her email is just one sentence long, which probably suggests that the urgency of the matter displaced the need for social niceties, i.e. greeting her recipient.

As stated earlier, the types of greeting used set the tone of the email. Starting an email with the name of the recipient is a matter-of fact opening. During an interview, one participant, Jean, said that only one staff member starts his email using this style and she would reciprocate the message by using the same style when responding to this person, as in Email 6.2 . 
Email 6.2 (CJ06b) NZ1

Subject: RE: UNDP Days 2011

Marcus

[message]

Jean

In email 6.2, Jean follows the same greeting format that Marcus used in his email to her, an observation that supports what she said about her usage of greeting during an interview: "if I receive an email with 'Hi Jean', I will write 'Hi so and so' but if I receive an email with just my name, I follow the sender's style”. Instead of her usual greeting 'Hi + first name', she begins the email with just his first name, which could suggest that she chooses to challenge the face sensitivities of her recipient.

It is interesting to note that the choice of greetings used varied from individual to individual. For example, one interviewee, Susan, said that she uses only the greeting word ' $\mathrm{Hi}$ ' and would not use 'Hi guys' when addressing many recipients. Another interviewee, Jean, said that it is very important to acknowledge her recipients' language for greeting. So she would use 'kia orana' if she corresponded with her clients from the Cook Islands. In the data collected, only one email started with the Maori greeting as in Email 6.3.

Email 6.3 (SA125) NZ1

Subject: Te Reo

Kia ora koutou katoa

[message]

Email 6.3 was sent to all staff when the CEO encouraged her staff members to use te reo Maori to greet potential clients who had Maori heritage. To set an example, she greeted her recipients with the Maori greeting 'Kia Ora koutou katoa'.

In spite of the preference expressed by ninety-three percent of those surveyed that it is important to end an email with a sign off, 41 percent of the emails did not have a closing expression. This shows that what people say they do is quite often different from what they actually do. Table 6.4 shows the types of closing expressions used in emails in Company NZ1. 
Table 6.4 Types of closing expressions in email in Company NZ1

\begin{tabular}{lcc}
\hline Types of closing expressions & Number of emails & Percentage (\%) \\
\hline No closing expression & 253 & 41 \\
First name only & 54 & 9 \\
Initial of first name only & 36 & 1 \\
Closing expression only & 6 & 25 \\
Closing expression + first name & 150 & 8 \\
Closing expression + initial of first name & 53 & 10 \\
Thanks + first name & 59 & $\mathbf{1 0 0}$ \\
\hline Total & $\mathbf{6 1 1}$ & 6 \\
\hline
\end{tabular}

Fifty-nine percent of the messages ended with some sort of closing expression. 'Cheers' (29\%) was the most common followed by 'Thanks' (12\%) and 'Regards' (1\%). These closing expressions were usually followed by the first name or the initial of the first name. Fifteen percent ended their emails with only the writer's first name or initial of the first name. Only four messages contained expressions which signal expectation ('See you soon') and affection ('Lots of love'): these messages were written by Susan to Jacinta who was having a vacation in The States. Closer analysis shows that when 'thanks' was used, sometimes it was used to express gratitude for a suggestion made or in anticipation of obtaining cooperation from the recipient, and this finding supports Waldvogel's (2005) analysis. However, there were also instances when 'thanks' was used as a sign off.

According to one interviewee, Jean, it is important to maintain the professional identity of the organisation and this can be achieved through formal closings. All interviewed agreed that it is courteous to sign off even though they have a signature block. At Company NZ1, the signature block consisted of the company's logo, the sender's full name, position in the organisation, telephone contact number, fax number, sender's email address and the company's mission statement. According to another interviewee, John, the signature block which is generated automatically would therefore be removed manually when writing to his colleagues as part of a chain of conversation. 
The following two emails are typical examples of the greetings and closings used by this CofP.

Email 6.4 (SS06) NZ1

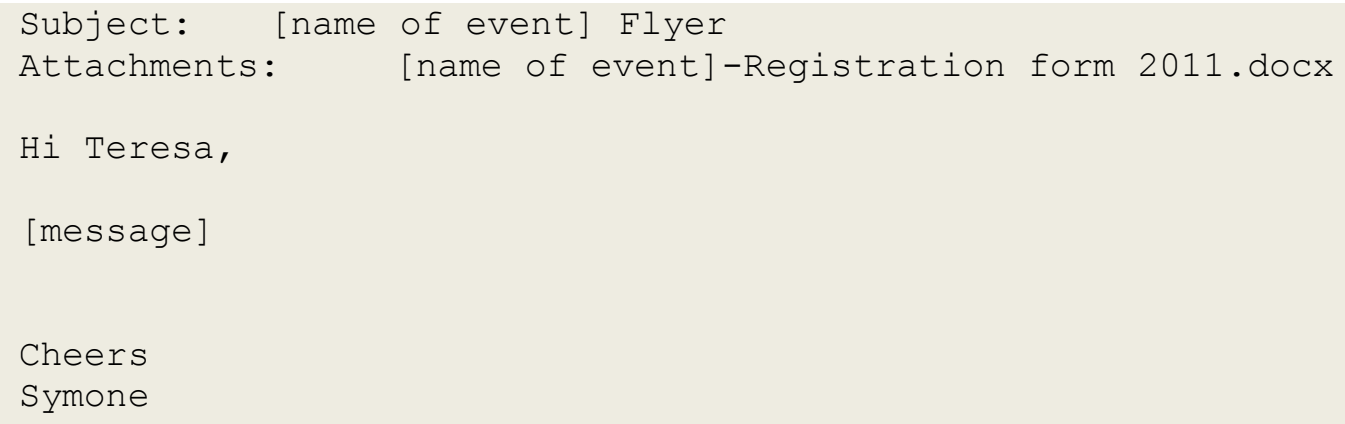

While the writers of emails 6.4 and 6.5 use the informal greeting ' $\mathrm{Hi}+$ first name', the choice of signing off differs. Although both emails are signed off informally, email 4.3 is signed off with 'cheers + first name' and email 4.4 is signed off with 'cheers + initial'. This informality probably indicates the generally collegial nature of the staff members' working relationship and it also possibly suggests the egalitarian nature of New Zealand society. It could also be an indication that staff members are attending to the face sensitivities of their colleagues.

In summary, the analysis of my email dataset shows that the most common greeting and closing form used are ' $\mathrm{Hi}+$ first name' and 'Cheers + first name' respectively. While most interviewed said that they like to receive emails which have a greeting and closing, they are not upset when the features are not present. They generally agreed that when an email becomes a chain of an on-going conversation, they would exclude the greeting and closing features. This view seems to be supported by the actual email dataset. 


\subsection{Use of greetings and closings in Company NZ2}

A total of 575 email messages were collected from Company NZ2 (see Section 3.5.4). Table 6.5 shows frequencies in the use of greetings and closings in emails.

Table 6.5 The use of greetings and closings in emails in Company NZ2

\begin{tabular}{lccccccccc}
\hline $\begin{array}{l}\text { Greeting } \\
\text { and } \\
\text { Closing }\end{array}$ & \multicolumn{2}{c}{$\begin{array}{c}\text { New or Primary } \\
\text { Message }\end{array}$} & \multicolumn{2}{c}{$\begin{array}{l}\text { Chain emails } \\
\text { (follow-up } \\
\text { messages) }\end{array}$} & \multicolumn{2}{c}{ Total } & \multicolumn{2}{c}{ Chi-square test } \\
\cline { 2 - 11 } & No & $\%$ & No & $\%$ & No & $\%$ & X2 & df & $\begin{array}{c}\boldsymbol{p} \\
\text { value }\end{array}$ \\
\hline No greeting & 212 & 55 & 116 & 62 & 328 & 57 & & & \\
Greeting & 176 & 45 & 71 & 38 & 247 & 43 & 2.815 & 1 & .0 .93 \\
No closing & 183 & 47 & 121 & 65 & 304 & 53 & & & \\
Closing & 205 & 53 & 66 & 35 & 271 & 47 & 15.581 & 1 & $.000^{*}$ \\
\hline Total emails & $\mathbf{3 8 8}$ & $\mathbf{1 0 0}$ & $\mathbf{1 8 7}$ & $\mathbf{1 0 0}$ & $\mathbf{5 7 5}$ & $\mathbf{1 0 0}$ & & & \\
\hline
\end{tabular}

Unlike Company NZ1, staff members in Company NZ2 tended not to include a greeting when writing new $(55 \%)$ or chain (62\%) emails. A chi-square test demonstrates that there is no statistically significant association between the kinds of email greeting (with a greeting, or without a greeting) and the two types of emails (new or chain emails) ( $\chi 2(d f=1)=2.815$, $p=.093)$. There are two possible reasons for the infrequent use of greeting in Company NZ2. Participants interviewed cited the high number of emails received daily as a reason for not including a greeting in routine emails. Another reason is the demands of the work in this workplace. However, messages to distant colleagues in Company NZ2 always contained a greeting. A rather different distribution is noted in the use of closing in new or primary messages when compared to chain messages, with staff members more likely to include a closing in new emails (53\%) rather than chain emails (35\%). A chi-square test reveals a statistical difference in the use of closing $(\chi 2(d f=1)=15.581, p=.000)$.

About 80 percent of those surveyed agreed or strongly agreed that it is important to start a message with a greeting or person's name and to end an email with a sign off. However, that did not match the actual emails collected from the participants since only 43 percent of the emails started with a greeting and 47 percent of messages ended with a closing. Most people interviewed agreed that the form of greeting used and the level of formality depends on the person they communicate with and the tone of the email that they received. Two staff members interviewed considered writing ' $\mathrm{Hi}$ ' a waste of time and another found it an 
annoyance when people write 'Hi guys'. Another participant reported that niceties such as greetings and closings are sacrificed due to the volume of emails received daily. From the interviews, it was found that the common greeting used was ' $\mathrm{Hi}$ '. Casual greetings such as 'Hi mate' and 'Hey mate' were used when addressing close colleagues. As with Company NZ1, all interviewees agreed that any form of greetings will be dispensed with when the email becomes a thread of conversation and this view is supported by the email dataset.

Table 6.6 shows the types of greetings used in emails in Company NZ2. The table shows that there was no consistency in the pattern of greeting used in the emails. Sometimes participants used greetings, and sometimes they did not, and when a greeting was used, the types of greeting used varied.

Table 6.6 Types of greeting in email in Company NZ2

\begin{tabular}{lcc}
\hline Greeting types & Number of emails & Percentage (\%) \\
\hline No Greeting & 328 & 57 \\
Greeting word only & 24 & 4 \\
First name only & 28 & 5 \\
Greeting word + first name & 189 & 33 \\
Thanks + Name & 6 & 1 \\
\hline Total & $\mathbf{5 7 5}$ & $\mathbf{1 0 0}$ \\
\hline
\end{tabular}

Fifty-seven percent of the messages did not have a greeting. Further analysis shows that messages without greeting fell into one of the following categories: a reply or follow-up message; a forwarded message; or a brief note or a message made to an appended attachment. When the email contained a greeting, the common greeting used was ' $\mathrm{Hi}+$ first name' (33\%). When addressing multiple recipients, formal greetings such as 'good morning' or 'good afternoon' or the informal greeting 'Hi All' or 'Hi Guys' were used. Closer analysis shows that a formal greeting was more likely to be used when greeting distant colleagues in this large workplace. Other types of greeting found in this dataset were the use of the recipient's first name only (5\%), greeting word only (4\%) and 'thanks + name' (1\%). When only the greeting word was used, the most popular word was 'Hey', followed by 'Hi'. Three emails used the term 'mate', suggesting that the writer attempted to enhance the solidarity 
of the relationship with the recipient and rapport building. Email 6.6 is one example when the term 'mate' was used to address the recipient.

Email 6.6 (CP44b) NZ2

Subject: $R E$ :

Thanks mate

In Email 6.6, Patrick uses the term 'mate' to address his superior which could be an indication of enhancing the solidarity of relationship between a subordinate and a superior.

Participants who were interviewed had different opinions on the use of the closing feature. Some participants who were interviewed said that they do not type their name as they feel that the signature block which states their name suffices while some felt that it is not necessary because the email format automatically shows the writer's name. In other words, writing the name is considered redundant, an opinion which supported Sherblom's (1988) view of a sign off as discussed in Section 6.2.2. Although seventy five percent of those surveyed strongly agreed or agreed that it is important to end an email with a sign off, suggesting that most people preferred to receive a message which ends with some sort of closing expressions, 53 percent of the messages did not contain any closing expression. This finding shows that people sometimes did not do what they said. Table 6.7 shows the types of closing expressions found in emails in Company NZ2.

Table 6.7 Types of closing expressions in email in Company NZ2

\begin{tabular}{lcc}
\hline Types of closing expressions & Number of emails & Percentage (\%) \\
\hline No closing expression & 304 & 53 \\
Closing expression only & 118 & 21 \\
First name only & 6 & 1 \\
Initial of first name only & 0 & 0 \\
Closing expression + first name & 112 & 19 \\
Closing expression + initial of first name & 5 & 5 \\
Thanks + first name & 30 & $\mathbf{1 0 0}$ \\
\hline Total & $\mathbf{5 7 5}$ & 1 \\
\hline
\end{tabular}


Email 6.9 (SS146) NZ2

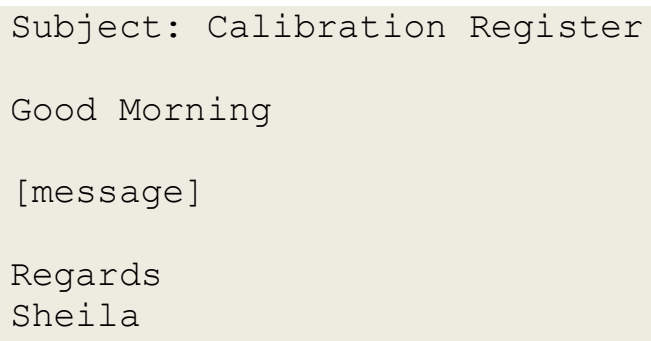

Email 6.10 (SK94) NZ2

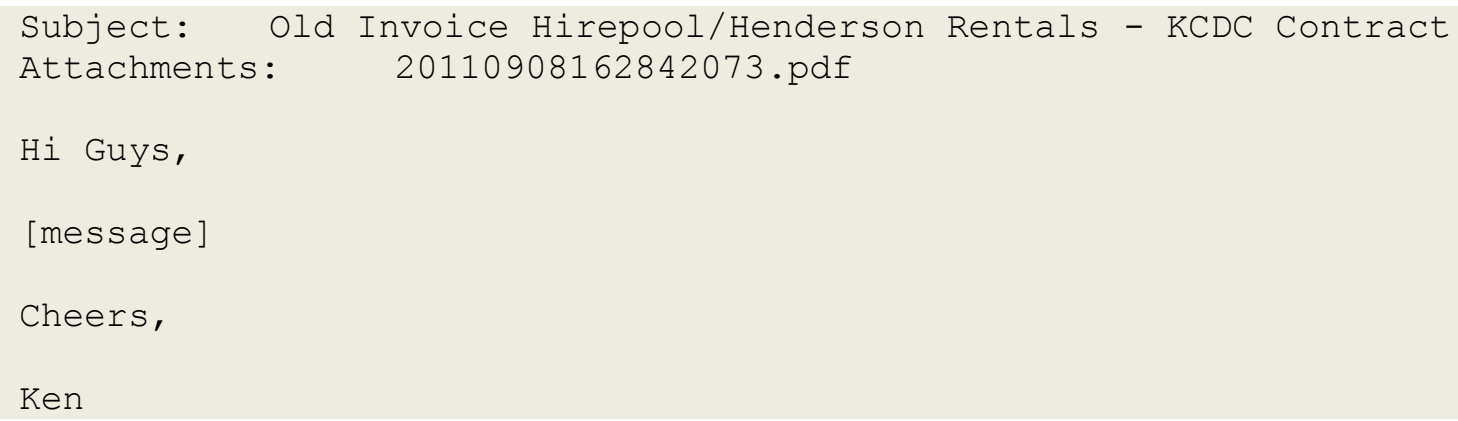

In Email 6.7, Ken uses the informal greeting ' $\mathrm{Hi}+$ first name' to address a close colleague and he signs off with the closing 'cheers + first name'. In contrast, in Email 6.8, Sheila greets her recipient, a distant colleague, 'Hi' followed by his name and signs off with a slightly more formal closing 'regards'. In Email 6.9, Sheila greets a group of distant colleagues 'good morning' and signs off with 'regards + name' while an informal greeting 'Hi Guys' is used when Ken addresses a group of close colleagues in Email 6.10. He signs off 'cheers' followed by his name. The use of the informal greeting and closing could be seen as a construction of social rapport and it also possibly indicates the egalitarian culture practised in New Zealand. In summary, the analysis of my email dataset in Company NZ2 shows that the informal greeting ' $\mathrm{Hi}+$ first name' is typically used when the emails are addressed to close colleagues where a formal greeting 'good morning' or 'good afternoon' is used when writing to distant colleagues in this large organisation. However, closing expressions such as 'cheers' and 'regards' followed by the recipient's name are popular choices. Similar to the responses obtained from Company NZ1, those interviewed in Company NZ2 said that it would be nice to receive an email with a greeting of some sort. And the majority of those interviewed 
agreed that greeting and closing are often dispensed with in subsequent emails, an observation supported by the findings of the email dataset.

\subsection{Use of greetings and closings in Company M}

As stated in Section 3.5.4, a total of 559 email messages were collected from Company M. Table 6.8 shows frequencies in the use of greetings and closings in emails.

Table 6.8 The use of greetings and closings in emails in Company M

\begin{tabular}{|c|c|c|c|c|c|c|c|c|c|}
\hline \multirow[t]{2}{*}{$\begin{array}{l}\text { Greeting } \\
\text { and } \\
\text { Closing }\end{array}$} & \multicolumn{2}{|c|}{$\begin{array}{c}\text { New or Primary } \\
\text { Message }\end{array}$} & \multicolumn{2}{|c|}{$\begin{array}{l}\text { Chain emails } \\
\text { (follow-up } \\
\text { messages) }\end{array}$} & \multicolumn{2}{|c|}{ Total } & \multicolumn{3}{|c|}{ Chi-square test } \\
\hline & No & $\%$ & No & $\%$ & No & $\%$ & $x^{2}$ & $d f$ & $\begin{array}{c}p \\
\text { value }\end{array}$ \\
\hline No greeting & 61 & 17 & 106 & 53 & 167 & 30 & & & \\
\hline Greeting & 299 & 83 & 93 & 47 & 392 & 70 & 80.705 & 1 & $.000^{*}$ \\
\hline No closing & 26 & 7 & 91 & 46 & 117 & 21 & & & \\
\hline Closing & 334 & 93 & 108 & 54 & 442 & 79 & 114.822 & 1 & $.000^{*}$ \\
\hline Total emails & 360 & 100 & 199 & 100 & 559 & 100 & & & \\
\hline
\end{tabular}

In the Company $\mathrm{M}$ dataset, 83 percent of new emails had a greeting compared to 47 percent chain emails $(\chi 2(d f=1)=80.705, p=.000)$. There is also a greater likelihood that participants include a closing expression in new $(93 \%)$ rather than chain $(54 \%)$ emails ( $\chi 2(d f=1)=114.822$, $p=.000)$. The results in Table 6.8 show that the difference is statistically significant. Closer analysis shows that more than two-thirds of the messages without a greeting $(76 \%)$ and a closing expression (72\%) came from the CEO's emails. Follow-up analysis (Table 6.9) which compares the two CEOs from Company M and Company NZ1 reveals that the New Zealand CEO (49\%) was more likely to include a greeting in her email than the Malaysian CEO (18\%) $(\chi 2(d f=1)=38.649, p=.000)$. Interestingly, chi-square result $(\chi 2(d f=1)=.074, p=.785)$ shows that they do not differ significantly in the use of closing which suggests that they both were not likely to sign off their emails. 
Table 6.9 The use of greetings and closings in emails by CEOs of Company NZ1 and Company M

\begin{tabular}{|c|c|c|c|c|c|c|c|}
\hline \multirow[t]{2}{*}{ Greeting and Closing } & \multicolumn{2}{|c|}{$\begin{array}{c}\text { Andy } \\
\text { Company NZ1 }\end{array}$} & \multicolumn{2}{|c|}{$\begin{array}{c}\text { Amir } \\
\text { Company M }\end{array}$} & \multicolumn{3}{|c|}{ Chi-square test } \\
\hline & No & $\%$ & No & $\%$ & $x^{2}$ & $d f$ & $p$ value \\
\hline No greeting & 120 & 51 & 128 & 82 & & & \\
\hline Greeting & 117 & 49 & 29 & 18 & 38.649 & 1 & $.000 *$ \\
\hline No closing & 125 & 53 & 85 & 54 & & & \\
\hline Closing & 112 & 47 & 72 & 46 & .074 & 1 & .785 \\
\hline Total emails & 611 & 100 & 575 & 100 & & & \\
\hline
\end{tabular}

In Company M, 87 percent of the participants interviewed agreed that it is courteous to start an email with a greeting and it is a cultural practice in this CofP that lower level staff members addressed their superiors formally. According to staff members interviewed, they address the CEO and the HODs with the title 'Mr' or 'Encik/ En' (Malay equivalent of 'Mr') or 'Ms' followed by their first name (for a Malay or Indian name) or surname (for a Chinese surname). They said that they 'sometimes' address their male superiors as 'Sir'. Such titles are used as a form of respect not only to superiors but also to colleagues who are more senior than the writer.

Table 6.10 shows the types of greeting used in emails in Company M. The table shows that there was a small variation in the pattern of greeting used in the emails.

Table 6.10 Types of greeting in email in Company $M$

\begin{tabular}{lcc}
\hline Greeting types & Number of emails & Percentage (\%) \\
\hline No Greeting & 167 & 30 \\
Titles or honorific epithets only & 6 & 1 \\
First name only & 38 & 7 \\
Greeting word + first name & 150 & 27 \\
Greeting word + title + first name & 187 & 33 \\
Greeting word + honorific epithets & 6 & 1 \\
Informal/ kinterm greeting & 5 & 1 \\
\hline Total & $\mathbf{5 5 9}$ & $\mathbf{1 0 0}$ \\
\hline
\end{tabular}

Seventy percent of the emails contained a greeting. Most messages began with the greeting word 'Dear', and close analysis shows that all messages from the lower level staff contained 
a greeting: the most common greeting 'Dear + first name' $(27 \%)$ was used if the email was directed to a peer; 'Dear + title + first name' (33\%) if the email was directed to superiors; or 'Dear + honorific epithet' $(1 \%)$ if the email was directed to the MD or his wife. When an email was sent to more than one recipient or a group of recipients as in the case of addressing all staff members, the popular greeting used was 'Dear All'. In contrast, messages from higher management to subordinates varied. Some contained greetings while some did not. If a greeting was present, the greeting was either 'Dear + first name' or first name only. Only seven percent of messages started with just the recipient's name. No messages began with only a greeting word or 'Thanks'.

When titles were used, they could be either in English or Malay, and when addressing their male superiors or a senior male colleague, the common greeting used was the title ' $\mathrm{Mr}$ ', 'Encik' (Malay equivalent of 'Mr') or 'sir'. The title 'Ms' or 'Cik' (Malay equivalent of 'Ms') was used when addressing female superiors or senior female colleagues. According to the CEO during an interview, this company is culture sensitive and using such forms of address signals one's respect for another. The use of 'title + first name' is similar to that found in Abdullah's (2003) and Alsree's (1997) studies; both studies are located in Malaysia. The honorific epithet 'Dato' is one of the highly regarded honorary titles conferred by a hereditary royal ruler of one of the nine Malay states in Malaysia. The wife of a 'Dato' is automatically referred to as 'Datin'. In my study, the only honorific epithets used were 'Dato' (referring to the MD) and 'Datin' (the MD's wife who was also one of the managers in the company) and the commonly used titles were 'Mr'/ 'Encik'/ 'En' and 'Ms'.

Informal forms of address used in spoken communications were also evident in some emails (1\%), as in Email 6.11. Some examples of the informal forms of address or kinterm used in spoken communications were 'kak' (Malay equivalent of sister) or 'aka' (Tamil equivalent of sister) when addressing a senior female colleague or 'abang' (Malay equivalent of brother) when addressing a senior male colleague. Sometimes the English kinterm 'bro(ther)' was used. Only one key participant (Kamal) used these spoken informal forms of address in his emails (four messages). In an interview, one staff member commented that such informal greetings are considered inappropriate in emails and she said that she avoids using such terms to portray a more professional image in the workplace. Consider the following example. 
Email 6.11 (SK18) M

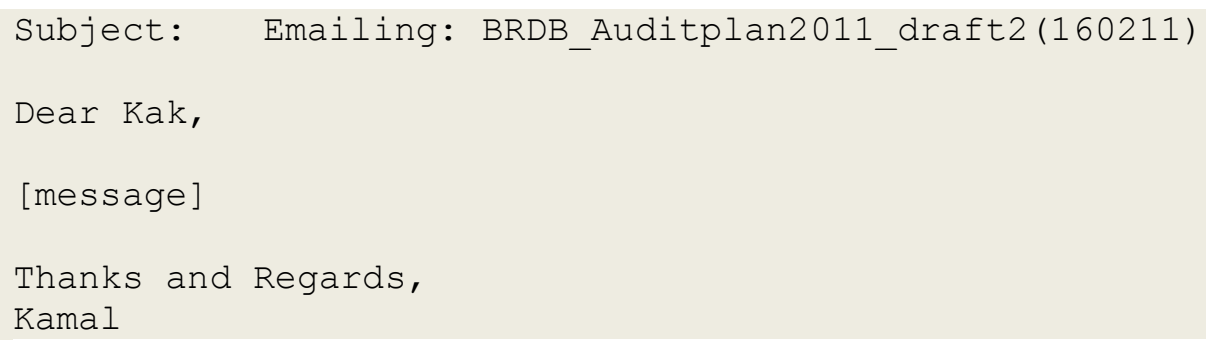

In Email 6.11, Kamal requests his colleague to check the audit plan that he has prepared. By greeting his colleague informally as 'kak', he could be doing two things: one, he attends to his colleague's identity face and sociality rights (Spencer-Oatey, 2000c); and two, he shows her respect. The second point supports Abdullah's (2003, p. 462) claim that "Malays prefer to interact with colleagues on a personal basis".

Table 6.9 shows 30 percent of the email messages contained no greeting. Email messages without greeting were usually from superiors and the majority were a follow-up message. Only three percent of messages upward were greetingless. Closer analysis shows that these were instant replies to an email chain. If a reply was sent a few hours later or the following day, a greeting and a closing were included in the email message, as in the examples in Emails 6.12a (SK64d) and 6.12b (SK64f).

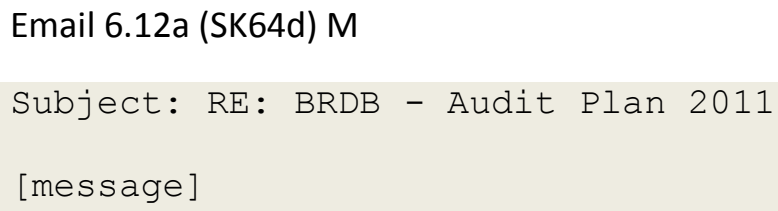

Both Emails 6.12a and 6.12b were part of an email chain and were authored by Kamal, a junior officer, to Encik Amir, the CEO. Kamal replies to the CEO on the same day and he does 
not include a greeting or a closing expression in Email 6.12a. I suggest two possible explanations for the omission: first, it is a quick reply to the CEO; and second, the urgency of the task takes precedence. As explained earlier, it is not a common practice for junior level staff members to omit a greeting and a closing expression in emails that they author to their superiors. This email is an exception to the common practice. In contrast, Email $6.12 \mathrm{~b}$ which is written three days later contains a greeting, a message and a closing expression.

Another interesting finding from Company $\mathrm{M}$ dataset is messages which were forwarded or messages with an appended attachment contained a greeting. In fact, the email not only included a greeting, it also had a message and a closing expression. Email 6.13 is one example.

Email 6.13 (SK62) M

Subject: Billing budget

Dear Bro Awang,

[message]

Thanks and Regards,

Kamal

Seventy percent of those surveyed agreed or strongly agreed that it is important to end an email with a sign off, indicating that that most people liked to receive a message which ended with some kind of closing expression. Consistent with the preference expressed by those surveyed to receive an email with a sign off, 79 percent of the messages had some sort of closing expression (see Table 6.8). Table 6.11 shows the types of closing expressions found in emails in Company M. 
Table 6.11 Types of closing expressions in email in Company M

\begin{tabular}{lcc}
\hline Types of closing expressions & Number of emails & Percentage (\%) \\
\hline No closing expression & 117 & 21 \\
Closing expression only (Thanks/ TQ/ Thank you) & 20 & 4 \\
First name only & 40 & 0 \\
Initial of first name only & 0 & 49 \\
Closing expression + first name & 274 & 0 \\
Closing expression + initial of first name & 0 & 19 \\
Thanks/ TQ/ Thank you + first name & 108 & $\mathbf{1 0 0}$ \\
\hline Total & $\mathbf{5 5 9}$ & 79 \\
\hline
\end{tabular}

Only a small percentage of the messages did not have a closing expression (21\%) or finished with just the writer's name (7\%). The majority of the emails ended with formal closing expressions (79\%), with 'Thanks and Regards'as the most popular followed by 'Regards' or 'Best Regards' and 'Thanks' / 'TQ'/ 'Thank you'. If the writers were from the top management, the closing expressions were either with or without the name of the writer. In contrast, writers who were subordinate to their recipients always included their name. It is interesting to note that an abbreviated version of 'Thank you', that is 'TQ', was popularly used by the staff members in this company. Closer analysis showed that 17 percent of messages ended with the closing ' $\mathrm{TQ}+$ name' or just ' $\mathrm{TQ}$ '. Twenty percent of these messages ended with just the closing expression 'TQ' and most of those were found in the CEO's emails. Another observation made is all messages contained a pre-programmed signature block which contained the writer's name, company logo, address and contact numbers. No messages concluded with 'Cheers' and the use of just the initial of first name were not practised in this CofP. 
MD. It only contains the honorific epithet as a greeting and concludes with 'TQ ('thank you') + name'. Email 6.17 is addressed to a peer. The email contains the greeting 'Dear + first name' and a closing 'Thanks \& Regards + name'.

In summary, the analysis of my email dataset in Company $M$ shows that greeting typically takes the following format: the greeting 'Dear + first name' was used when the emails were addressed to peers and to subordinates; the more formal greeting 'Dear + title + first name' was used when writing to superiors; the greeting 'Dear + honorific epithet' was used when writing to the MD or his wife; greetingless emails or emails addressing the recipients with just the first name were normally from the superiors. There are fewer variations of closing expression, with the common closing expressions being 'regards and thanks + name' and 'thank' ' $T Q$ '. Close analysis reveals that if the email becomes a chain conversation, the greeting and closing are sometimes dispensed with. However, this omission is only observed in the superiors' emails while subordinates (other than the exception discussed above) include both the greeting and closing in all emails directed upward even when the email is a long chain conversation. As in the responses obtained from the two New Zealand workplaces, those interviewed in Company $M$ said that it would be nice to receive an email with a greeting of some sort. This view is supported by the findings of the email dataset.

\subsection{Discussion}

The analysis above shows that the use of greetings and closing expressions as well as their types differed in the three workplaces. First, I present and discuss a summary of the frequencies in the use of greetings and closings in emails in the three workplaces. Next, I discuss the use of greetings and the types of greeting used, and finally, I present a summary of the use of closings and the types of closing expressions used.

As discussed in Sections 6.3, 6.4 and 6.5, a total of 611, 575 and 559 email messages were collected from Company NZ1, Company NZ2 and Company M respectively. Table 6.12 shows frequencies in the use of greetings and closings in emails and the results of the chi-square test that was performed to test the significance of the trends in the data in the three workplaces. 
Table 6.12 The use of greetings and closings in emails in Company NZ1, Company NZ2 and Company M

\begin{tabular}{|c|c|c|c|c|c|c|c|c|c|}
\hline \multirow{2}{*}{$\begin{array}{l}\text { Greeting } \\
\text { and } \\
\text { Closing }\end{array}$} & \multicolumn{2}{|c|}{ Company NZ1 } & \multicolumn{2}{|c|}{ Company NZ2 } & \multicolumn{2}{|c|}{ Company M } & \multicolumn{3}{|c|}{ Chi-square test } \\
\hline & No & $\%$ & No & $\%$ & No & $\%$ & $x^{2}$ & $d f$ & $\begin{array}{c}p \\
\text { value }\end{array}$ \\
\hline No greeting & 281 & 46 & 328 & 57 & 167 & 30 & & & \\
\hline Greeting & 330 & 54 & 247 & 43 & 392 & 70 & 86.605 & 2 & $.000^{*}$ \\
\hline No closing & 253 & 41 & 304 & 53 & 117 & 21 & & & \\
\hline Closing & 358 & 59 & 271 & 47 & 442 & 79 & 125.043 & 2 & $.000 *$ \\
\hline Total emails & 611 & 100 & 575 & 100 & 559 & 100 & & & \\
\hline
\end{tabular}

In Company NZ1 and Company M dataset, more than half of the email messages had a greeting (54\% and $70 \%$ respectively) and this compares with 43 percent of email messages in the Company NZ2 dataset. Likewise, a higher proportion of emails were signed off with a closing in Company NZ1 (59\%) and Company M (79\%) compared with Company NZ2 (47\%). A chi-square analysis indicates a statistical difference in the use of greeting ( $\chi 2(d f=2)$ $=86.605, p=.000)$ and closing $(\chi 2(d f=2)=125.043, p=.000)$ between the three companies. Follow-up analyses (Tables 6.13 and 6.14) that aim to examine which workplace was more likely to include a greeting and closing show that the three companies differ significantly from one another.

Table 6.13 Results for the Chi-square test for the use of greeting

\begin{tabular}{lccc}
\hline Comparison (raw data in brackets) & $\mathbf{\chi 2}$ & $\boldsymbol{d f}$ & $\boldsymbol{p}$ value \\
\hline Company NZ1 (330/611) vs. Company NZ2 (247/575) & 14.487 & 1 & $.000^{*}$ \\
Company NZ1 (330/611) vs. Company M (392/559) & 32.085 & 1 & $.000^{*}$ \\
Company NZ2 (247/575) vs. Company M (392/559) & 85.060 & 1 & $.000^{*}$
\end{tabular}

In order to avoid Type 1 errors, $p$-value was adjusted at .017 (.05/3; 3 is the number of comparisons)

Table 6.14 Results for the Chi-square test for the use of closing

\begin{tabular}{lccc}
\hline Comparison (raw data in brackets) & X2 & $\boldsymbol{d f}$ & $\boldsymbol{p}$ value \\
\hline Company NZ1 (358/611) vs. Company NZ2 (271/575) & 15.625 & 1 & $.000^{*}$ \\
Company NZ1 (358/611) vs. Company M (442/559) & 56.610 & 1 & $.000^{*}$ \\
Company NZ2 (271/575) vs. Company M (442/559) & 123.872 & 1 & $.000^{*}$
\end{tabular}

In order to avoid Type 1 errors, $p$-value was adjusted at .017 (.05/3; 3 is the number of comparisons) 
It is interesting to note that between the two New Zealand companies, participants in Company NZ1 were more likely to include a greeting (54\%) in the emails compared to 43 percent in Company NZ2 ( $\chi 2(d f=1)=14.487, p=.000)$. They were also more likely to sign off their emails $(\chi 2(d f=1)=15.625, p=.000)$.

Comparing the two New Zealand companies and the Malaysian company (Company M), it is noted that a higher percentage of email messages in Company $\mathrm{M}$ had a greeting (70\%) and a closing expression (79\%). Chi-square tests performed show that there are statistical differences in the use of greetings and closings (see Tables 6.13 and 6.14 for pair-wise comparisons). The results show that participants in the Malaysian company were more likely to include a greeting and signed off their emails compared to the participants in the two New Zealand companies.

All these results suggest that:

1. staff members in Company NZ1 places more emphasis on maintaining solidary relationships and doing collegiality through the use of greeting while the absence of a greeting in Company NZ2 tends to suggest that it is business first.

2. workplace culture appears to have some influence on the presence and absence of the use of greeting and closing, and as argued in Section 6.5, using a greeting in emails in the Malaysian company was not only a shared practice in the CofP, it is also a means of showing respect or doing deference.

Table 6.15 summarises the greeting types used in emails in the three workplaces, and shows that there was no consistency in the pattern of greeting used in the emails. Sometimes participants used greetings, and sometimes they did not. And when they used greetings, the types used varied. 
Table 6.15 Greeting types in Company NZ1, Company NZ2 and Company M

\begin{tabular}{lc|c|c|c|c|c}
\hline & \multicolumn{2}{c}{ Company NZ1 } & \multicolumn{2}{c}{ Company NZ2 } & \multicolumn{2}{c}{ Company M } \\
\cline { 2 - 7 } Greeting types & $\#$ & $\%$ & $\#$ & $\%$ & $\#$ & \% \\
\hline No Greeting & 281 & 46 & 328 & 57 & 167 & 30 \\
Greeting word only & 44 & 7 & 24 & 4 & 0 & 0 \\
First name only & 27 & 4 & 28 & 5 & 38 & 7 \\
Titles or honorific epithets only & 0 & 0 & 0 & 0 & 6 & 1 \\
\hline Greeting word + first name & 242 & 40 & 189 & 33 & 150 & 27 \\
Greeting word + title + first name & 0 & 0 & 0 & 0 & 187 & 33 \\
Greeting word + honorific epithet & 0 & 0 & 0 & 0 & 6 & 1 \\
Thanks + Name & 17 & 3 & 6 & 1 & 0 & 0 \\
\hline Informal greeting & 0 & 0 & 0 & 0 & 5 & 1 \\
\hline Total & $\mathbf{6 1 1}$ & $\mathbf{1 0 0}$ & $\mathbf{5 7 5}$ & $\mathbf{1 0 0}$ & $\mathbf{5 5 9}$ & $\mathbf{1 0 0}$ \\
\hline
\end{tabular}

If a greeting was used, the common greeting used in the two New Zealand workplaces was 'Hi + first name'. All the key participants from the two workplaces greeted their recipients with ' $\mathrm{Hi}$ ' which suggested that they were very informal with each other and with their other colleagues. It could also be a reflection of the more egalitarian New Zealand attitude to interpersonal relationships at work as found in other studies (Waldvogel, 1999, 2005, 2007). In addition, by using a greeting, the writers attended to the identity face and association rights (Spencer-Oatey, 2000b) of their recipients and subsequently enhanced rapport with them. Using symmetrical address forms such as first name alone further suggests solidarity and collegiality among staff members. The informal greeting ' $\mathrm{Hi}$ ' was used regardless of the status of the recipient as in Email 6.18.

Email 6.18 (SK70) NZ2

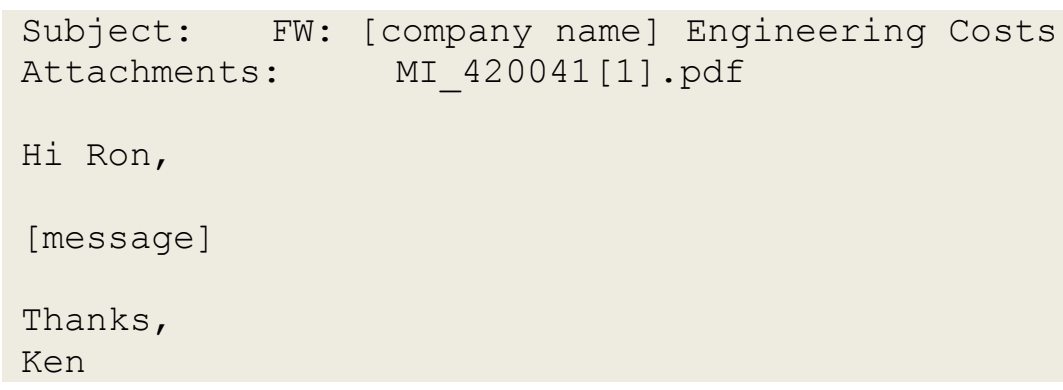


In Email 6.18, Ken uses the informal greeting ' $\mathrm{Hi}+$ first name' to address his superior, the General Manager, and he signs off with the closing 'thanks + first name'. Using an informal greeting suggests that Ken is attending to his superior's identity face and association rights.

In contrast, no participants in the Malaysian workplace used a casual greeting such as 'hi' or greeted their superior by first name in the emails. The common greeting used was 'Dear + first name' when writing to peers. The use of first name alone to refer to one's superior would probably be interpreted as being rude or showing disrespect to the superior. The common greeting preferred was'Dear + title + first name' when writing to superiors or someone senior, and for recipients who had an honorific title, the greeting used was 'Dear + honorific epithet'. My findings support the studies by Abdullah (2003, p. 333) and Alsree (1997) who find that "Malaysian subordinates tended to adhere to Malaysian asymmetrical conventions". Superiors, on the other hand, used either one of these formats: 'Dear + first name'; first name only; no greeting included. And as stated in the discussion above (see Section 6.4), it is a cultural practice in this company that superiors were addressed formally by the lower level staff members. It was a sign of showing respect and deference. Thus, it is not surprising that 'Dear + title + first name' and 'Dear + honorific epithet' were found in the Malaysian data but not in the two New Zealand data.

While using the greeting 'Dear + first name' was a norm in the Malaysian workplace, using 'Dear' in the New Zealand workplaces was regarded as formal and showed negative rather than positive politeness to the recipient (Waldvogel, 2007). Using 'Dear' in New Zealand could also convey deference rather than solidarity and collegiality. The high usage of 'Dear' as a greeting in the Malaysian data is similar to that of Crystal's (2006) British study of 500 personal emails where he finds that two-thirds of his emails contained this greeting.

The use of ' $\mathrm{Hi}$ ' in the New Zealand data or 'Dear' in the Malaysian data as a greeting was found in emails directed to a single recipient and multiple recipients. This observation is in contrast with other studies. In Nickerson's (1999) study, a greeting was included only if the message was intended for a single recipient and its use was uncommon in emails to multiple recipients. In my study, the greetings ' $\mathrm{Hi}+$ first name' in the New Zealand data or 'Dear + first name' and 'Dear + title + first name' in the Malaysian data were found in emails to single recipients. When emails were directed to two or more staff members, in the New Zealand data, the greetings ' $\mathrm{Hi}+$ All' or 'Hi Guys' were used. In the Malaysian data, the 
greeting 'Dear + All' was used to address multiple recipients. No messages in the Malaysian data had used the address term 'guys'.

As with the use of greetings, the use of closings in the three workplaces differed. The contrast is presented in Table 6.16.

Table 6.16 Types of closing expressions in Company NZ1, Company NZ2 and Company M

\begin{tabular}{|c|c|c|c|c|c|c|}
\hline \multirow[t]{2}{*}{ Types of closing expressions } & \multicolumn{2}{|c|}{ Company NZ1 } & \multicolumn{2}{|c|}{ Company NZ2 } & \multicolumn{2}{|c|}{ Company $\mathrm{M}$} \\
\hline & \# & $\%$ & \# & $\%$ & $\#$ & $\%$ \\
\hline No closing expression & 253 & 41 & 304 & 53 & 117 & 21 \\
\hline Closing expression only & 6 & 1 & 118 & 21 & 20 & 4 \\
\hline First name only & 54 & 9 & 6 & 1 & 40 & 7 \\
\hline Initial of first name only & 36 & 6 & 0 & 0 & 0 & 0 \\
\hline Closing expression + first name & 150 & 25 & 112 & 19 & 274 & 49 \\
\hline Closing expression + initial of first name & 53 & 8 & 5 & 1 & 0 & 0 \\
\hline Thanks/ TQ/ Thank you + first name & 59 & 10 & 30 & 5 & 108 & 19 \\
\hline Total & 611 & 100 & 575 & 100 & 559 & 100 \\
\hline
\end{tabular}

Emails without closing expressions mirrorred that of greetingless emails in the New Zealand workplaces. Comparing the three workplaces, Company NZ2 had the highest number of emails (53\%) without closing expressions compared to the other two workplaces. This finding concurs with Sherblom's $(1988$, p. 44) study which regarded closing expressions as "informationally redundant" due to the format of email.

If a closing was used, the common expressions in the two New Zealand workplaces were 'Cheers + first name' followed by 'Regards + first name'. In Company NZ1, the preference was 'Cheers + name' while there was an equal distribution of the two patterns in Company NZ2. Only one percent of messages concluded with 'Regards + name' in Company NZ1. In contrast, no participants in the Malaysian workplace used the closing expression 'Cheers'. Instead more formal closing expressions were found in the data such as 'Regards + first name' and 'Thanks and Regards + first name', and the use of closing expressions was more common in emails directed upward. This finding mirrors the findings in Sherblom's (1988) study. In his study of email files of a large American organisation, he finds that organisational 
hierarchy influenced the use of signatures. His findings reveal that subordinates were more likely to sign their emails upwards than emails directed to peers.

Another contrast observed is the use of the initial of first name. According to Crystal (2006, p. 108), the "use of initialism - either the initial letter of just the first name, or of both the first name and surname" is common in informal interaction. In my study, the use of initial of first name in various patterns such as 'Cheers + initial of first name' or initial of first name only, was common in the data found in Company NZ1 but did not occur in Company M. Less than one percent of messages with this pattern were found in Company NZ2.

Although the two New Zealand workplaces suggested a more egalitarian New Zealand culture, they differed in the use of the pattern of closing expressions. The use of 'Cheers + first name' and initial of first name in Company NZ1 could indicate a more collegial atmosphere in the workplace. Three factors contributed to this collegial atmosphere and close interpersonal relationships among the staff members: they were a small CofP; the staff members knew each other not just as colleagues but also as friends; and they were based in the same office. In contrast, the closing expression used in Company NZ2 was 'Regards + first name' which seemed to suggest greater formality.

One interesting observation noted in the Malaysian data is the use of the unconventional abbreviation of the word 'TQ' which means 'Thank you' in the closing feature. This abbreviated word 'TQ' occurred in 17 percent of messages and it was used with or without the first name of the writer. A closer analysis shows that the CEO signed off with the word 'TQ' without the first name more often than any of the other key participants. I conducted an informal survey to find out if New Zealanders and other nationalities are familiar with this abbreviated word. Interestingly, the results from this informal survey showed that this word is known and popularly used by Malaysians only.

The presence or absence of greeting and closing expressions and the types used contribute to the construction of collegiality in the workplace and how they impact on rapport building. The tendency to use both greetings and closings directed to distant colleagues in Company NZ2 suggests the importance of constructing relationship and solidarity between colleagues and signals attempts at minimising social distance. This concurs with the findings of Waldvogel's (2005) comparative study of a manufacturing plant and an educational 
organisation. She finds that 67 percent of distant colleagues were greeted with some kind of greeting formula or acknowledged by name.

The use of greeting was dispensed with when the email was a reprimand as found in Emails 6.19 and 6.20 from Company NZ1 and Company NZ2 respectively. In contrast, the use of 'Dear' as a greeting was found in the email with the same communicative function in the Malaysian data, as in Email 6.21. Emails 6.19, 6.20 and 6.21 are discussed in the following paragraphs. See Appendix 6 for the full version of these three emails.

Email 6.19 was written by Andy to four staff members who were involved in a dispute over car park spaces.

Email 6.19 (SA31) NZ1

Subject: RE: Car parks

All

[message]

In Email 6.19, Andy addresses her staff members who were involved in a dispute over car park spaces as 'All' instead of a friendly ' $\mathrm{Hi}$ '. The omission of a sign off in this email could be interpreted as she appears to have no intention in building rapport with her subordinates. The address 'All' and the fact that she does not sign off this email further suggest that she probably has adopted a rapport-neglect orientation or rapport-challenge orientation (Spencer-Oatey, 2000b) with her subordinates.

In the next example, Email 6.20 below was sent by Megan to all the staff members in the company to voice her annoyance over poor attendance at a training session.

Email 6.20 (SM16) NZ2

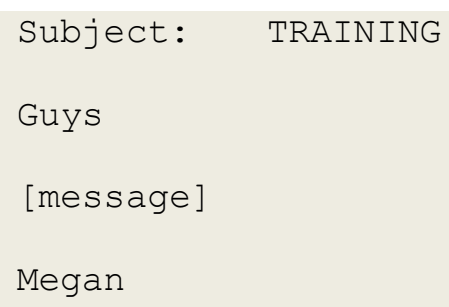


Megan starts her email with 'Guys' unlike her usual 'Hi Guys' in emails sent to multiple recipients. She stresses that she is 'pretty annoyed' and emphasises that 'it is not too much to ask that [she was] notified in time to make the necessary changes'. In addition she concludes the email with her name only. The absence of a friendly greeting and closing show that Megan appears as not eager in building rapport with the staff members. She could be seen as holding a rapport-neglect orientation or rapport-challenge orientation (SpencerOatey, 2000b) with the staff members.

Email 6.21 was sent by Chandran, the HOD to his subordinate who did not comply with the guidelines when preparing a report.

Email $6.21(\mathrm{SCO5}) \mathrm{M}$

Subject: BRDB-Procument of Suppliers Non Project

Dear [name],

[message ]

Chandran

Unlike Emails 6.19 and 6.20 from the New Zealand workplaces which start in a 'let's get down to business' tone, i.e. 'All' or 'Guys', in Email 6.21, Chandran includes a greeting 'Dear + name' in the email to his subordinate. However, it can be argued here that Chandran has no intention to build rapport with his subordinate because the use of greeting is just the norm practised in this workplace. It is obvious from the tone of the email that Chandran is unhappy with the quality of the report his subordinate has prepared and he makes it very clear to him that the CEO 'does not speak well of [his] report'. He also stresses 'I told you to put down in your report ... yet you did not do so' and '... your report does not cover the areas ...' which further indicate that he is rather annoyed with his subordinate. In addition, he signs off with just his name instead of his usual closing 'Thanks and regards'. From this evidence, it can be concluded that Chandran could be seen as not interested in fulfilling the rapport building goal or maintaining a harmonious work relationship with his subordinate on this occasion. In interview data staff members commented that it was common for them to receive emails with red font and capital letters from top management. Two interviewees said that it was the top management's exclusive rights to write such emails and labelled 
these emails as "red emails". This email thus demonstrates that Chandran, the HOD, took advantage of his position to write a direct email which he ends with a command in capital letters 'READ THEM' to his subordinate.

In conclusion, from the discussion presented in this chapter, it can be argued that the use of greeting and closing contributed to the construction of collegiality in the workplace and had an impact on rapport building. Although greeting is optional and a closing expression is redundant (as discussed in Section 6.2.2), they (greetings and closing expressions) are considered important for the following reasons:

- They inject a personal touch to the medium which was once regarded as a lean medium (Daft \& Lengel, 1986) because email lacks the non verbal social cues present in face to face communication; and

- They can contribute to the construction of solidarity and can be used to build and maintain rapport (Spencer-Oatey, 2000b) with the recipients;

- They can be used as a strategy to attend to the identity face and association rights (Spencer-Oatey, 2000b) of the recipients; and

- They can signal and indicate respect and deference toward the recipients, in the case of the Malaysian context.

\subsection{Summary}

This analysis has demonstrated that the use of greetings and closings in the three workplaces differed. The use of informal greeting and closing in Company NZ1 and Company NZ2 showed the general attitude toward interpersonal relations at work in New Zealand workplaces as compared to the workplace in Malaysia where status and title were given more emphasis. The use of first name and informal greeting in the two New Zealand workplaces, regardless of status and seniority also indicated the egalitarian nature of New Zealand society. The widespread use of the informal and friendly ' $\mathrm{Hi}$ ' in Company NZ1 and Company NZ2 further signalled solidarity and collegiality, as compared to the formal use of 'Dear' followed by title and/ or honorific epithets which denoted deference and formality as found in the data in the Malaysian workplace. Also in Company $M$, greeting and closing were more likely used by subordinates when writing to superiors or people of higher status. 
However, even within the same city (Wellington) in the same country (New Zealand), the choice in the use of greetings and closings in the two New Zealand workplaces showed a contrast. Emails in Company NZ1 showed that the staff members displayed a more collegial atmosphere with the use of 'Cheers' as a closing expression. In contrast, staff members in Company NZ2 preferred a more formal closing expression, 'Regards'. One possible reason that attributed to the difference is that staff members in Company NZ2 were more socially distant because of the size of the company, the nature of business of the organisation and the fact that not all the staff members were based in the same location.

Having presented a discussion on the use of greetings and closings in the three workplaces, in the next chapter, I focus on the various types of linguistic features found in request emails. 


\section{Chapter 7: Requests - constructing power relations and rapport}

\subsection{Introduction}

Previous research has shown that there are cross-cultural differences in how requests are worded (Blum-Kulka, 1982; Blum-Kulka \& Olshtain, 1989; Hendriks, 2010; Kankaanranta, 2005; Lee, 2011; Wierzbicka, 2003; Yli-Jokipii, 1994). This chapter discusses the various discourse strategies used by New Zealand and Malaysian email writers in realising the communicative function of requests. My primary goal is to investigate the strategies used by email writers at different levels in the hierarchy in the three different workplaces to reduce the face threat of their requests and to gain compliance and cooperation from their recipient(s).

Requests provide a rich source of information about workplace relationships. Firstly, they are face-threatening acts which could impair work relationships because of their delicate nature; secondly, managers and subordinates construct their workplace status through the forms of request they use; thirdly, the communicative function of request was identified as the second most important function performed by email in all three workplaces; and fourthly, email can be used to transmit both transactional and affective information, that is "rich" information which challenges the rich media theory suggesting that email is a lean medium (Daft \& Lengel, 1984). In other words, requests provide a favourable context for investigating how email writers negotiate workplace relationships (i.e. build rapport) and do power at the same time.

In analysing request emails, I addressed the following questions:

- Are requests realised differently by people of different status and if yes, how?

- Are requests realised differently by people in different workplaces and if yes, how and why?

- Are there different patterns in relation to different workplace status?

- What strategies are used by people to strengthen or mitigate their requests in the three workplaces? 
This chapter is divided into six sections. First, I survey definitions of request and indicate the definition used in the analysis; in the second section, I examine types of requests; in the third, fourth and fifth sections, I discuss request emails directed downward, upward and laterally respectively; and lastly, I present a summary.

\subsection{Definition of requests}

As discussed in Chapter 5, a Speech Act Theory (Searle, 1969, 1976) approach is the basis for categorising the email dataset in my study. While some researchers make a distinction between directives and requests, others do not (Abdullah, 2003; Craven \& Potter, 2010; Ho, 2009; Kankaanranta, 2005; Parry, 2013; Vine, 2001). In the paragraphs that follow, I discuss the distinction between the terms 'directives' and 'requests' and the reason for adopting the latter and not the former term in the present thesis.

In classic speech act theory, directives are defined as "attempts ... by the Speaker to get the Hearer to do something" (Searle 1976:11). However, Searle's definition does not make a distinction between acts that require a physical response (directives) and those that require a verbal response (elicitation), unlike Sinclair and Coulthard (1975, pp. 40 - 41, 50 -51) who do. Sinclair and Coulthard (1975) make that distinction in their examination of teacher-pupil language. They clearly distinguish directives as the teacher getting the "pupil to do but not say something"(Sinclair \& Coulthard, 1975, p. 28). Craven and Potter (2010, p. 420 ,original emphasis) define directive as "an action where one participant tells another to do something". A directive, given the label 'control act' is defined as "an attempt to get someone to do something" (Vine, 2009, p. 1396), and in the workplace context a directive is an attempt by the manager to get a staff member to complete an action. Vine $(2001, p .71)$ also claims that there is an expectation of compliance and none of the directives are to be refused: "with directives, the speaker is of higher status and the hearer has no right of refusal. Requests, on the other hand, involve a situation where the speaker is of equal or lower status, and the hearer, generally has more right of refusal". Having examined the different definitions of a directive, it is clear that all agree that when a directive is issued, the purpose is to get the hearer to accomplish something.

While Vine (2001) makes a distinction between the terms directives and requests, I use the term 'requests' to refer to both directives and the speech act of making requests. As stated 
in Section 5.3, the term 'requests' is preferred because the participants in the workplaces refer to the speech act which requires someone to do something as requests rather than directives although it is obvious that a request from a superior to a subordinate would expect compliance and cannot easily be refused. In other words, the generic term 'requests' is preferred even though it is obvious that when the request is made downward, it has greater force (Vine, 2001).

Researchers in written business communication have offered various definitions of requests. Blum-Kulka, House and Kasper (1989a, p. 11) define request as a 'pre-event act' which expresses "the speaker's expectation of the hearer with regards to some prospective action". This definition is somewhat confusing as it limits requests to just performing an act. Kankaanranta (2005, p. 364) defines a request as "an expression of a need with which the writer wants the reader to perform either a verbal or a physical act". This definition is not suitable in the context of my research as it does not include requests which include a cognitive response. So for my study, I adopted Yli-Jokipii's (1994, p. 60) broad definition of request which states that a request is "a message which seeks a physical, verbal or cognitive act [response] on the part of the recipient". Ho's $(2009$, p. 3) definition of request incorporates the "recipient's need for compliance on grounds of institutional, professional and personal motivators such as necessity, duty and goodwill". Because I was unable to collect information about the participants' institutional, professional or personal motivators, the definition proposed by Yli-Jokipii (1994) seems more appropriate for my study. In addition, this definition seems suitable because in the workplace, requests are not limited to performing a physical act (response) alone. Requests also include the writer's attempt to obtain information (verbal) and advice (cognitive). See Chapter 5 for the number of emails in each sub-category for each workplace.

Yli-Jokipii (1994) provides useful and practical advice when identifying requests: some requests have specific tags or features which assist in labelling them as requests, such as the politeness marker 'please', the imperative form, and the interrogative form. The following four examples clearly fulfilled the specific markers of requests. The letter/s in brackets indicate/s the workplace. NZ1 shows that the message comes from Company NZ1, NZ2 signals Company NZ2 and M represents Company M. 
1. [peer to peer]

Could you please forward the Audit Plan that you did? (M)

2. [superior to subordinate]

Please add to the monthly report. (NZ2)

3. [peer to peer]

Please print 2 copies the attached reports for AC Meeting and pass it to Mr.

Chandran. (M)

4. [superior to subordinate]

Angela can you please circulate this to all staff and ask them to fill in any detail they know and/or add any new contacts in. (NZ1)

These examples illustrate the tags identified by Yli-Jokipii (1994): they were realised in the interrogative (Examples 1 and 4 ) and imperative (Examples 2 and 3 ) forms and all included the use of 'please'.

In the next section, I discuss the classification of types of requests in terms of the purpose of the emails and the form of the requests. Adopting a "pragmalinguistic" approach, Leech (2007, p. 195) notes that it is possible to identify some requests by attending just to the linguistic resources (such as syntactic, morphological and lexical items) used to realise them. However, in many cases, the functions of the emails as requests cannot be fully interpreted without considering contextual factors. This is especially true in the case of indirect requests where context is crucial in identifying their communicative function. It is also important to consider contextual factors when determining the degree of directive force expressed by a request. In such cases, a "socio-pragmatic" approach (Leech, 2007, p. 195) is required, and this is the focus of discussion in Sections 7.3.2, 7.4, 7.5 and 7.6. 


\subsection{Classification of types of requests}

\subsubsection{Purposes of requests}

As stated in Section 7.2, I classified the broad category of requests into three sub-categories: requests for action, requests for information and requests for advice. These sub-categories fulfilled the definition adopted from Yli-Jokipii (1994) as they indicate to the recipient the type of response desired: a physical act (requests for action), a verbal act (requests for information), and a cognitive act (requests for advice).

I shall now turn to my discussion of the three sub-categories of requests.

\subsubsection{Requests for action}

Requests for action refer to messages having the purpose of getting the recipient to perform an action such as to arrange for a meeting, to write a report, to contact someone or to coordinate with other department members, and they formed one of the largest group of requests in the present dataset (see Sections 5.3.1, 5.3.2 and 5.3.3). Abdullah (2003) claims that this category of request has a greater potential threat to the recipients' face than the other two categories of request. She finds that these messages have the following features: (1) they tend to have fewer than average primary recipients; and (2) they have a higher inclusion of salutations, personal closings and signature blocks. My analysis shows that more than half of the total emails performing this function contained a greeting and a closing. The following examples illustrate this category of requests and supports Abdullah's (2003) description.

1. [superior to subordinate]

Could you change that to the new name? (NZ1)

2. [peer to peer]

Please process this. (NZ2)

3. [superior to subordinate]

As per my conversation with Dato this morning, kindly arrange to dispose the safe. (M)

4. [subordinate to superior]

Can you add the (name of client) into our Sponsorship financial sheet for $\$ 30,000$ probable for 2011 column please? (NZ1) 


\subsubsection{Requests for information}

Requests for information ask for specific information such as names, contact numbers, figures, explanations and status of reports. They are generally considered routine in workplaces and are "part of the job", and they are, therefore, considered the least facethreatening to the recipients (Abdullah, 2003, p. 342).

Abdullah (2003) claims that messages requesting information in workplace contexts are more likely to be authored by peers than by subordinates and superiors. However, my data show that superiors from Company NZ1 and Company M contributed the highest number of emails of this category. As CEOs in their respective companies, they were the decisionmakers and many things needed to be clarified and solved immediately before problems arose. Hence, requesting information to facilitate decision making was necessary. See Sections 5.4.1 for details. Typically this type of request was expressed as interrogative, occasionally ellipsed, as in example 5 below. The following examples illustrate this category of requests.

1. [superior to subordinate]

Can you let me know what we paid [name of client] last year as the [name of event] coordinator? (NZ1)

2. [superior to subordinate]

Could you please advise of the plant number for Peter's Saw so we can request a replacement capex for it. (NZ2)

3. [superior to subordinate]

Any idea who WILL BE IN OFFICE ?? (M)

4. [peer to peer]

This is the only information I can find of an example for out of hours can you elaborate? (M)

5. [peer to peer]

Any idea's who the driver is? (NZ2)

6. [subordinate to superior]

Who is Tim? (NZ2) 


\subsubsection{Requests for advice}

Requests for opinion, advice or suggestion are as the phrase suggests, requesting advice. Here are some examples of requests for advice in my datasets.

1. [subordinate to superior]

... any suggestions for a speaker - looking for a successful female entrepreneur that we can put them in touch with? (NZ1)

2. [subordinate to superior]

What is the next step? (NZ2)

3. [subordinate to superior]

Kindly advise on the next course of actions. (M)

4. [peer to peer]

Do you need me to send this today or would it be better to send on Friday last thing in order for the email to be in their email boxes first thing Monday morning. (NZ1)

While most requests for advice were relatively easy to identify, this was not always straightforward. Sometimes it was difficult to decide whether it was a request for action or a request for information. Where the distinction was not clear, two analytic strategies were used: first, an examination of the response from the recipient; and second, a consideration of the observation notes and my interpretation as a participant observer. The following first message of a chain email (Email 7.1) is one example where the request could be interpreted as either a request for action or a request for information.

Email 7.1a (CS18a) NZ1

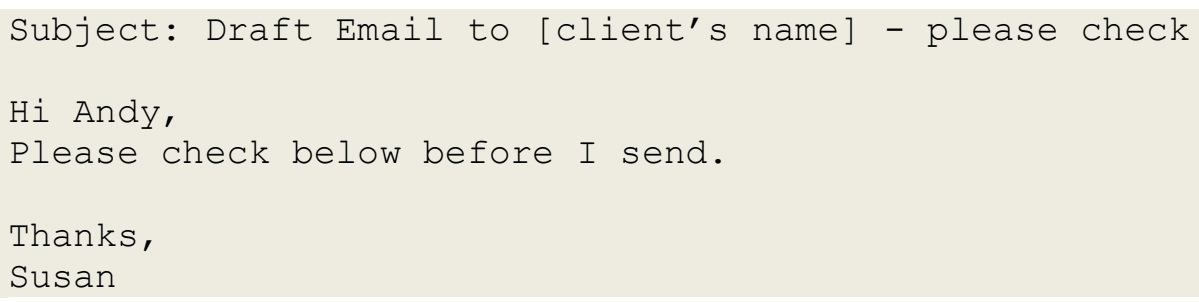


Email 7.1b (CA18b) NZ1

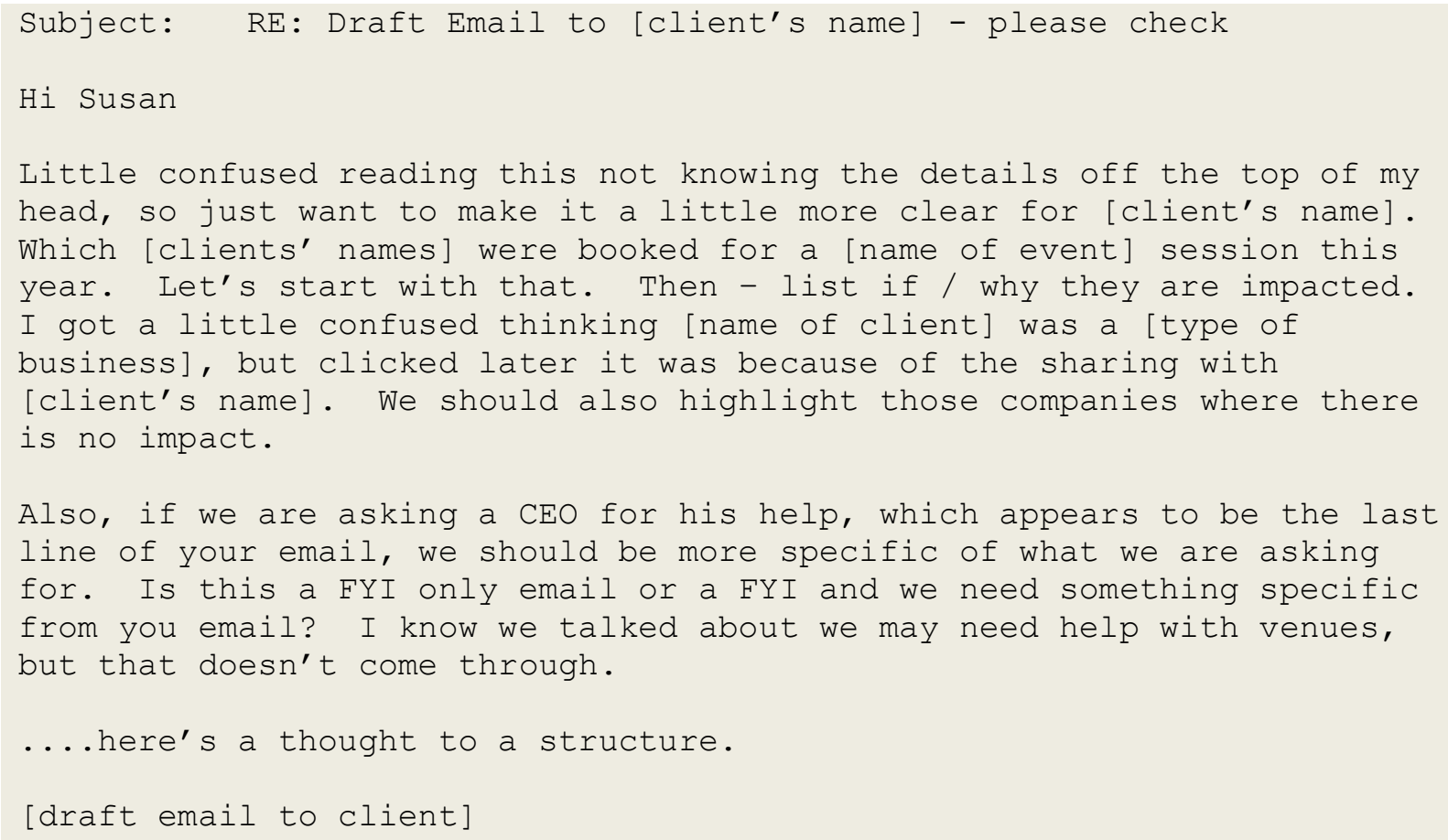

This is a chain email (Email 7.1a and 7.1b) between Andy and Susan from Company NZ1. In Email 7.1a, Susan requests Andy to check the email she drafted before she sends it to a client. This request clearly signals a request for action but it also seems to request a verbal response. If the draft needs to be changed and revised, it needs to be explained but if it does not need any changes, this view has to be communicated to Susan. Requests of this type were classified as requests for actions because some actions needed to be taken in order to fulfil the request made even though the act of 'checking' could be communicated verbally. In Email 7.1b, Andy gives some suggestions 'let's start with that', 'We should also highlight those companies ...', and '... should be more specific of what we are asking for'. To end the email, she includes a revised email '... here's a thought to a structure'. Andy's reply to Susan's request clearly shows that some kind of action on Andy's part was taken. Therefore, the recipient's response helps to identify the request type. 
Another difficulty of classification concerned the word 'advise' as in the phrase 'please advise ...'. This was sometimes classified as a request for information and other times as a request for advice. The following are some examples of requests which contain the verb 'advise'.

1. [subordinate to superior]

Kindly advise on the next course of actions. (M)

2. [subordinate to superior]

Please advise when I can close my month end and finish my report pending your decision on moving figures as per our discussion. (NZ2)

3. [superior to subordinate]

Could you please advise of the plant number for [name]'s Saw so we can request a replacement capex for it. (NZ2)

4. [peer to peer]

Can you advise who will be fixing the [name of event] online registration? (NZ1)

5. [superior to subordinate]

Can you advise the average day to complete both [types of processes]? (NZ2)

I classified examples (1) and (2) as requests for advice. They differ from the ordinary requests for information, not in their linguistic form alone, but in the context in which the requests are made, i.e. they ask the recipient for their opinion or suggestion rather than just information seeking. In example (1), Sharifah requests her CEO's advice. She wants to know what she needs to do next because her CEO commented that her (Sharifah) time cost exceeded the budget allocated in the project she was working on. Therefore, this example is a request for advice. Similarly, in example (2), Patrick requests his superior's advice on the problem they had discussed so that he can complete his report. So example (2) is another illustration of request for advice. In contrast with examples (1) and (2) which require the recipients to give their opinion, examples (3) to (5) are information seeking requests. They ask for specific information such as number (as in example (3)), name (as in example (4)) or duration (as in example (5)). 
After classifying requests using mainly linguistic features as the basis of the categorisation, in the following section, I discuss the three basic forms in which requests are realised, adopting Leech's (2007) socio-pragmatic approach, i.e. paying attention to contextual factors.

\subsubsection{Forms of requests}

Making requests of any kind may be deemed face-threatening (Brown and Levinson, 1987) and can have negative implications on solidarity and collegiality among staff members in a workplace environment. The person to whom the request is addressed can interpret requests as intrusive encroachment on freedom of action, or even as a sign of exercising power on the part of the person who makes the requests.

Requests can be classified as direct or indirect by drawing on Brown and Levinson's (1987) classification of bald-on-record and other politeness strategies (see section 2.4.1). Different types of requests are recognised as having a different degree of pragmatic force which constitutes different levels of potential threat to the recipient's face needs. For example, Abdullah (2003, p. 341) claims that a request for information carries less threat to the recipient's face as compared with a request for action, and she provides one possible reason for this: providing information is seen as part of the job for many employees. EconomidouKogetsidis (2011), however, finds that non-native speakers' email requests (particularly requests for information) in academic settings were characterised by significant directness and such emails were perceived as discourteous and impolite in power-asymmetrical situations. These emails were categorised as offensive for their failure to consider sociopragmatics strategies such as "forms of address, degree of formality, degree of directness, complementary closes, presence and amount of mitigation" (Economidou-Kogetsidis, 2011, p. 3194)

Abdullah (2003) also claims that a request for advice has a different implication as it depends on the status of the recipient; if the request is made upward, i.e. from a subordinate to a superior, it could be deemed more threatening to the requester's face for two reasons: first, it is because the recipient does not have the obligation to give a response; second, it could imply that the requester does not have the ability or the capability to carry out a specific task. On the other hand, if the request is made downward, i.e. from a superior to a 
subordinate, it could put the recipient in a very tricky situation:if the recipient gives an honest opinion, it may threaten the requester's (i.e. the superior's) face, however, no response may indicate insubordination to the superior (Abdullah, 2003, p. 341). She concludes, therefore, that requests for information can be viewed as less face-threatening compared to requests for advice while requests for action can be interpreted as the most face threatening act. While I agree with Abdullah that there are different degrees of request force for each type of request, I find her justification that an honest opinion may threaten the superior's face somewhat problematic. Giving an honest opinion may not necessarily threaten the recipient's face as it depends on various factors including the type of advice and the relationship with the recipient.

Analysis of requests in both speech and writing can pose potential problems between form and function (Blum-Kulka et al., 1989a; Yli-Jokipii, 1994). Some researchers claim that requests made in the imperative and interrogative forms are classified as direct requests while requests made in other forms represent indirect requests (Kankaanranta, 2005). However, according to Holmes (1999), a directive realised in an interrogative form or declarative form may have the same force as one expressed in the imperative form. She asserts that context rather than form plays the most important role in determining the force of a directive.

Requests can be realized in three syntactic forms: imperatives, declaratives and interrogatives, which are also referred to as mood choices in Halliday's (2004) interpersonal metafunction. Table 7.1 summarises the number of requests realised in the three syntactic forms and non-clausal units (see Section 7.3.3) for the three workplaces. These are discussed in turn in the following sections.

Table 7.1 Summary of distribution of requests realised in imperative, interrogative, declarative mood and non-clausal units

\begin{tabular}{lcccccc}
\hline \multirow{2}{*}{ Form } & \multicolumn{2}{c}{ Company NZ1 } & \multicolumn{2}{c}{ Company NZ2 } & \multicolumn{2}{c}{ Company M } \\
\cline { 2 - 8 } & No & $\mathbf{\%}$ & No & $\mathbf{\%}$ & No & \% \\
\hline Imperative mood & 61 & 21 & 100 & 39 & 261 & 68 \\
\hline Interrogative mood & 176 & 60 & 140 & 55 & 58 & 15 \\
\hline Declarative mood & 54 & 19 & 15 & 6 & 64 & 16 \\
\hline Non-clausal units & 0 & 0 & 0 & 0 & 1 & $>1$ \\
\hline Total & $\mathbf{2 9 1}$ & $\mathbf{1 0 0}$ & $\mathbf{2 5 5}$ & $\mathbf{1 0 0}$ & $\mathbf{3 8 2}$ & $\mathbf{1 0 0}$ \\
\hline
\end{tabular}




\subsubsection{Imperative mood}

The imperative mood is considered the most direct form of imposition in requests (Leech, 1983, p. 119). Brown and Levinson (1987) view the imperative form as the most direct and explicit example of a 'bald-on record' speech act because the threat of the hearer losing 'face' is very high. According to Leech (1983), although an imperative violates the TactMaxim which concerns politeness in asymmetrical contexts, it may not be deemed impolite. Brown and Levinson (1987), however, argue that if both the writer and recipient view the request as urgent and task-oriented, i.e. related to routine work, the request would not be viewed as impolite. In my study, the imperative mood was used by both superiors and subordinates when making requests to each other. Consider the following examples of requests which were related to routine work.

1. [peer to peer]:

Remove him quick before more come up. (NZ2)

2. [superior to subordinate]:

Check the email I sent you. (NZ1)

3. [superior to subordinate]:

please email me any of the Mieco QP progress reports. (M)

4. [peer to peer]:

Kindly email or cc to me if you get the response from them. (M)

5. [subordinate to superior]:

Please authorise the above order. (NZ2)

6. [subordinate to superior]:

Kindly review the Billings and Collections Budget for 2011. (M)

In the six examples given above, the requests were related to routine tasks and were less likely to pose any face-threat to the recipients. Examples (1) and (2) are realised as bald-on record imperatives which are classified as direct requests. Examples (3) to (6) are realised with the base form of imperative and used with 'please' or 'kindly'. All examples are classified as direct requests.

The imperative mood was used in all three workplaces when making requests but to differing extents. Imperatives can also occur with 'please', 'kindly' or 'let'. A quantitative 
analysis of the occurrence of imperative mood in realising requests was conducted to provide insights into the preferred form for making requests. Table 7.2 below shows the distribution of the use of imperatives alone, with 'please', 'kindly' and 'let' in the three workplaces.

Table 7.2 Distribution of imperative mood and its various forms

\begin{tabular}{lccccccc}
\hline \multirow{2}{*}{ Form } & \multicolumn{2}{c}{ Company NZ1 } & \multicolumn{2}{c}{ Company NZ2 } & \multicolumn{2}{c}{ Company M } \\
\cline { 2 - 8 } & No & $\mathbf{\%}$ & No & $\mathbf{\%}$ & No & \% \\
\hline Bare imperative & 21 & 34 & 14 & 14 & 33 & 13 \\
\hline 'Please' + imperative & 30 & 49 & 83 & 83 & 179 & 69 \\
\hline 'Kindly' + imperative & 0 & 0 & 0 & 0 & 45 & 17 \\
\hline 'Let' imperative & 10 & 16 & 3 & 3 & 4 & 2 \\
\hline Total Imperatives in the dataset & $\mathbf{6 1}$ & $\mathbf{2 1}$ & $\mathbf{1 0 0}$ & $\mathbf{3 9}$ & $\mathbf{2 6 1}$ & $\mathbf{6 8}$ \\
\hline
\end{tabular}

The imperative mood was less preferred when making requests in Company NZ1 (21\%) while more than one-third (39\%) of the requests made in Company NZ2 were realised in this form. These findings were similar to that found in Waldvogel's (2005) and Yli-Jokipii's (1994) studies. In Waldvogel's (2005) New Zealand study, she finds that only one-fifth of the directives were realised in the imperative form while Yli-Jokipii's (1994) study of American, British and Finnish business writing shows that few requests were in the form of imperative. Company $\mathrm{M}$ had the highest percentage (68\%) of requests realised in the imperative mood, which contrasts with Abdullah's (2003) study of workplace emails in Malaysia. Abdullah (2003) finds that only 11.3 percent of requests were made in the imperative form. Close examination of my data shows that request emails upward in Company $\mathrm{M}$ were realised in the imperative form. Ho (2009) also finds that the imperative form was preferred when making upward requests in his Hong Kong data. He argues that using a high level of directness suggests that subordinates make their request explicit to save the superior's time and effort in decoding the message.

As Table 7.2 shows, a high percentage of the bare imperative was used in Company NZ1 (34\%), followed by Company NZ2 (14\%) and Company M (13\%). Imperative with 'please' was preferred by the participants in Company NZ2 (83\%) and Company M (69\%) while almost half of the requests were realised in this form in Company NZ1 (49\%). See section 7.3.3 for a discussion of whether 'please' has a mitigating effect. 
In the present dataset, the marker 'kindly' used with imperatives was only found in the Malaysian data. Seventeen percent of the imperatives were realised in this form and close analysis shows that this form was used by subordinates writing upward and between equals when making requests. There were 18 instances when making requests upward, three downward and 24 instances between equals. According to House and Kasper (1980), the marker 'kindly' plays down the impact of the request made. Their study of Finnish and Swedish writers shows that the Finns used the word 'kindly' more, which could be attributed to the transfer of the equivalent word ystävällisesti in Finnish business language. Similarly, I argue that the word 'kindly' could be attributed to the translation of the Malay equivalent 'harap'. It could be interpreted as a more polite way of making requests especially when the request was made upward or between colleagues of equal status.

Another form of imperative is the 'let' imperative. This form of imperative was less preferred by the participants in all three workplaces. Table 7.2 shows that Company NZ1 had the highest usage of this form compared to the other two workplaces. Sixteen percent of the requests were realised in the 'let' + pronoun + imperative in Company NZ1 while Company NZ2 and Company M had three percent and two percent respectively.

In the present dataset, 'let' imperative collocated most frequently with the pronouns 'us' and 'me'. There were a total eight instances of 'let' + us + verb and nine instances of 'let' + me + verb in the three workplaces. It is interesting to note that all nine instances of 'let' + 'me' + verb collocated with the verb 'know'. 'Let us/me know' actually means 'tell me' and 'let us/ me have' means 'give me', depending on the context in which the message was written. The following three examples from Company NZ1 illustrate the explanation above:

1. [subordinate to superior] Let me know when it suits to sort it.

2. [superior to subordinate]

Let me know what [time] that meeting is and I'll come with you.

3. [peer to peer]

If you have a good news story, let's have it! 
In example (1), Susan requests Andy to tell her which day and time to meet to discuss a potential problem. Similarly, in example (2), Andy requests Susan to inform her the meeting time scheduled with a client so that she can also attend the meeting. Lesley, in example (3), on the other hand, requests her colleagues to give her write-ups of their events.

\subsubsection{Interrogative mood}

Requests can also be realised using the interrogative form. Holmes (1983, p. 103) suggests two major forms of interrogatives: modal and non-modal forms. I adopted Waldvogel's (2005) sub-division of modal interrogatives because this has proven useful when making a comparison between native speakers and non-native speakers of English. The sub-division is as follows:

i. requests realised with the modal 'can'

ii. requests realised with other modals such as 'could', 'would' or 'may'

One reason Waldvogel (2005) offers for this distinction is that different forms of language are class dependent. She claims that the multi-cultural staff from varied backgrounds from the manufacturing plant might not share the same linguistic knowledge as her other group of participants from the educational organisation with its "high percentage of tertiary and largely university educated staff who were more at ease with more subtle uses of the language" (Waldvogel, 2005, p. 214). In my data, the participants in the Malaysian workplace were non-native speakers of English and they might not share the same linguistic knowledge as the native-speakers in the two New Zealand workplaces. Apart from the CEO, all participants interviewed reported that Malay is their first language and they had limited exposure to English. They only learned English when they started their formal education when they were seven years old. 
Alsree (1997) argues that requests realised with other modals (such as 'could' and 'would') have a more softening effect and appear more polite than the form 'can'. The following are some examples from my dataset:

1. [subordinate to superior]:

May I please use my company car (plate number) to go to Turangi this weekend? (NZ2)

2. [superior to subordinate]:

Could you change that to the new name? (NZ1)

3. [superior to subordinate]:

Could you do that for this board meeting? (NZ1)

Besides using a modal, another type of interrogative form is the use of Wh-questions or Yes/No Questions which were categorised as non-modalised interrogatives in my study. This type of interrogative is usually quite direct and it is typically used when making requests for information. The following are some examples of requests realised with the Wh-questions:

4. [peer to peer]

Whats that fella name/ number? (NZ2)

5. [peer to peer]

Who should this go to now? (NZ2)

6. [superior to subordinate]

Sorry to trouble you on this old story..... do you have a soft copy of the workings that I did a few years ago? (M)

7. [subordinate to superior]

Do you want me to organise a meeting (NZ1)

Examples (4) and (5) are realised as Wh-questions while examples (6) and (7) are Yes/No questions. Usually such interrogatives look for a specific, one-word answer. In example (4), Patrick wants to know the name of the driver and/ or vehicle number. Similarly, in example (5), Patrick wants to know who he should forward an invoice to. Both emails were directed to his colleague of equal status. Clearly, (4) and (5) are requests for information. 
In examples (6) and (7), the expected answer is 'yes' or 'no'. However, in example (6), a simple 'yes' would not be sufficient. If the recipient does not have a soft copy of workings, a 'no' answer would suffice. But, if the answer is 'yes', then the author of the email would expect the recipient forward him the soft copy. So if the recipient has a soft copy of the workings but merely answered 'yes' and does not forward a copy to the requester, it could be interpreted as unprofessional, blatant insubordination (since the author of the email is the CEO of the company) or it could be treated as a joke. Therefore, (7) can be classified as a request for information and (6) as a request for action if the answer to the question is 'yes'.

Table 7.3 below shows the distribution of the use of interrogatives and its various forms in the three workplaces.

Table 7.3 Distribution of requests realised in the interrogative and its various forms

\begin{tabular}{lccccccc}
\hline Interrogative Mood & \multicolumn{3}{c}{ Company NZ1 } & \multicolumn{2}{c}{ Company NZ2 } & \multicolumn{2}{c}{ Company M } \\
\cline { 2 - 8 } & No & \% & No & $\%$ & No & $\%$ \\
\hline Modal Interrogatives with 'can' & 89 & 51 & 73 & 52 & 11 & 19 \\
\hline Interrogatives with other modals & 23 & 13 & 7 & 5 & 3 & 5 \\
\hline Non-modalised interrogatives & 64 & 36 & 60 & 43 & 44 & 76 \\
\hline Total interrogatives in the dataset & $\mathbf{1 7 6}$ & $\mathbf{6 0}$ & $\mathbf{1 4 0}$ & $\mathbf{5 5}$ & $\mathbf{5 8}$ & $\mathbf{1 5}$ \\
\hline
\end{tabular}

Interrogatives made up the largest percentage of requests in Company NZ1 (60\%) and Company NZ2 (55\%). My finding supports Waldvogel's (2005) finding. Her research showed that 42 percent of directives were realised in the interrogative form. In contrast, the interrogative mood was the least preferred request form in Company M. Only 15 percent of requests were realised in this form. A number of participants, particularly the lower level subordinates in Company $\mathrm{M} I$ interviewed remarked that it was easier to write in the imperative form than other linguistic forms. This result is similar to that found in Yli-Jokipii's (1994) data of real life and textbook company letters. She found only ten percent of interrogative requests in her data.

Although the interrogative 'can' form is generally considered as conventionally indirect, Waldvogel (2005) argues that this form suggests a great familiarity between the interactants. The modal form 'could' which Brown and Levinson (1987) term a negative politeness strategy is regarded as more polite and indirect. In Company NZ1 and Company NZ2, the 
majority of interrogatives were modalised and more than half of these were realised in the modal 'can' form (see Table 7.3). In contrast, only 19 percent of the interrogatives in Company $\mathrm{M}$ took the form of the modal 'can'. Only one instance of the use of the modal 'may' is found in the data in Company NZ2. The request was made by Patrick to his superior to seek permission to use the company car for personal use (see Example (1) above).

Participants in Company M used non-modalised interrogatives such as Wh-Questions and Yes/No Questions most frequently to perform the function of requesting responses and information from each other, and they formed the largest percentage (76\%) of interrogatives. A number of potential reasons for the common use of Wh-Questions and Yes/No Questions in Company M can be offered. Yes/No Questions by design allow the writers to present their propositions to the recipients for elaboration and confirmation. Similarly, Wh-Questions aim to elicit specific details from the recipients. These types of interrogatives can be employed to obtain definitive answers which are crucial to ensure that problems are solved efficiently and quickly. In contrast, less than half of the interrogatives in Company NZ1 (36\%) and Company NZ2 (43\%) were realised in this form. It is interesting to note that the findings in these three workplaces are in marked contrast to Waldvogel's research. Her study showed only three percent of non-modalised interrogatives in her corpus. One possible reason for this contrast is messages realised in this form in my study were information seeking requests and were considered less face-threatening.

\subsubsection{Declaratives}

Requests in varying degrees of directness can be realised in a declarative form. It is also noted that requests in this form are the least face-threatening requests although they can be as direct as the imperative form. The explicitness of a request in the declarative form depends on the context in which it is produced (Holmes, 1999).

Declaratives allow the writer/speaker to express a suggestion about whether something should or should not be done rather than giving an order to someone to perform/ do something and can be expressed in 'want' and 'need' statements. Ervin-Tripp's (1976) research on 'need' and 'want' statements show that in a work-related environment where roles, responsibilities and obligation are clearly defined, the use of 'need' and 'want' 
statements by a superior implied compliance on the part of a subordinate. Supporting ErvinTripp's (1976) claim, Chen (2006, p. 44) states that unmitigated 'want' statements can be deemed impolite as they suggest a "coercive tone".

The following are some examples of requests realised in a declarative form:

1. [peer to peer]

We need to release the retention through the claim invoice request and then request a credit not*. (NZ2) [ ${ }^{*}$ note]

2. [superior to subordinate]:

I will leave this one to you. (NZ1)

3. [peer to peer]:

You will need to set aside at least two hours for the audit itself and a similar amount of time to generate the [name of repots] from the findings. (NZ2)

In all three examples provided above, what is requested is as explicitly stated as in the imperative form. In example (1), Ken writes to another colleague from a different department. He frames the request in the declarative mood 'we need to ...' to gain cooperation from his peer. Using the pronoun 'we' Ken also emphasises that both he and his peer (the recipient) would be doing the doing the task together. Also, the inclusive pronoun 'we' aligns him with his peer, and reduces the illocutionary force of the request. In example (2), Andy tells Marcus to work out with his colleagues who would attend the seminar that the company had already paid for. Although she says 'I will leave this one to you', it is clear that she wants Marcus to ensure that there is a representative from the company as the modal 'will' seems to intensify the illocutionary force of the request. In example (3), Sheila, the Senior Administrator, requests her colleagues who are all managers, to allocate 'at least two hours' for the actual audit exercise and 'a similar amount of time' to prepare audit reports. In Company NZ2, it is important that each department conforms to the industry standards and failing an audit test will cause negative repercussions to the company. Therefore, although the request is made in the declarative mood, it has the same force as the imperative form. In this context, the modal 'will' can be interpreted as 'must' ('you must set aside ...') and it clear that Sheila wants her colleagues to comply with her request. 
Consider the following requests, from Company NZ1, which are also realised in the declarative mood:

4. [superior to subordinate]

If you could follow up with [name of client], that would be great.

5. [peer to peer]

Perhaps the three of us could get together later today to discuss the dates - $2 \mathrm{pm}$ ?

6. [peer to peer]

Teresa l'd like to include a sponsors logo and or picture if possible.

In contrast with the three examples given above, the last three examples, (4) to (6), are mitigated. In example (4), Andy uses the modal auxiliary 'could', a more indirect form than 'I'd like', and she further softens the request by a word of encouragement 'that would be great'. Similarly, in example (5), the request is hedged with 'perhaps' and a question mark to indicate tentativeness. Although Lesley proposes a time for the discussion to take place, the question mark could be interpreted as a suggestion and that the time is negotiable. Like example (5), example (6) is hedged with 'if possible' that softens the directness of the request (I'd like).

Table 7.4 below shows the distribution of the use of declaratives in the three workplaces.

Table 7.4 Distribution of requests realised in the declarative mood

\begin{tabular}{lccccccc}
\hline \multirow{2}{*}{ Form } & \multicolumn{2}{c}{ Company NZ1 } & \multicolumn{2}{c}{ Company NZ2 } & \multicolumn{2}{c}{ Company M } \\
\cline { 2 - 7 } & No & $\%$ & No & $\%$ & No & $\%$ \\
\hline Declarative mood & 50 & 94 & 15 & 100 & 61 & 95 \\
\hline Declarative + 'please' & 4 & 7 & 0 & 0 & 3 & 5 \\
\hline Total declaratives in the dataset & $\mathbf{5 4}$ & $\mathbf{1 9}$ & $\mathbf{1 5}$ & $\mathbf{6}$ & $\mathbf{6 4}$ & $\mathbf{1 6}$ \\
\hline
\end{tabular}

In Company NZ1 and Company M, the proportion of requests made in the declarative form was similar. Declaratives account for 19 and 16 percent in Company NZ1 and Company M respectively. In contrast, requests of this form accounted for only six percent in Company NZ2. There is one possible reason why declaratives were least frequently used to perform the function of requesting in Company NZ2. Because declarative 'need' and 'want' statements can imply compliance and perceived as forceful in a work-related environment, participants in Company NZ2 who frequently communicated with their distant colleagues 
may want to reduce its direct nature of requesting by opting not to use this mood clause. Not using the declarative mood for requesting could also help create rapport between the writer and the recipient in this large organisation.

In the following section, a discussion of the use of 'please' in the three syntactic forms and non-clausal units is presented.

\subsection{3 'Please' in mood clauses}

'Please' is widely researched in many contexts and in various cross cultural environments. Some of these include existing studies on the use of 'please' in telephone service discourse (Economidou-Kogetsidis, 2005), student-university communication (Economidou-Kogetsidis, 2011; Hartford \& Bardovi-Harlig, 1996; Usó-Juan \& Martínez-Flor, 2008), children's discourse (Wootton, 1984), varieties of English (Sato, 2008) and various request environments (House, 1989; Lee-Wong, 1994b). According to House's (1989, p. 107) work on 'please' and bitte (German equivalent), 'please' is more frequently used in "standard situations" in which interlocutor's role and obligation is clearly determined (e.g. between a seller and a customer) and the degree of imposition is low. Lee-Wong's (1994b) study on 'please' and qing (Chinese equivalent) in three different countries (People's Republic of China, Melbourne and Singapore), on the other hand, shows that the illocutionary force of qing closely relates to the context and its value as a politeness marker is essentially important in some cases. The use of 'please' in workplace email requests has also received much attention and there have been debates whether it is used as an attempt to mitigate the illocutionary force of requests or otherwise (Alsree, 1997; Waldvogel, 2005).

Although some researchers label 'please' as a request marker (House, 1989; Lee-Wong, 1994a) or a politeness marker (Brown \& Levinson, 1987), other researchers find it problematic (e.g. Waldvogel, 2005). Some researchers claim that the use of 'please' is seen as a strategy to gain cooperation and functions as a face-saver in a work related environment while Waldvogel (2005, p. 177) argues that 'please' has a "chameleon-like" effect because its illocutionary force changes depending on the context in which it is used. While Alsree (1997) claims that 'please' is used to mask a directive in the writer's attempt to get the recipient to cooperate, Paramasivam (2011) argues that the use of 'please' in aviation discourse serves as an appeal to the controller to comply with the directive issued 
by the pilot during critical moments. The use of 'please' in the following examples from my dataset illustrates the point made.

1. [superior to subordinate]

Please email me the draft....not to worry... will work around the details that you give me..... (M)

2. [peer to peer]

Please have names to me by COB Friday 12th August. (NZ2)

In example (1), Amir, the CEO, requests Geetha to email him the details he needs to prepare a speech for the company's annual dinner that night. Even though it is important that he receives the information because the speech needs to be prepared, the use of 'please' in conjunction with the assurance 'not to worry' appears to soften the illocutionary force of the request and serves as an appeal for cooperation. In contrast, the use of 'please' in example (2) does not have the effect of softening the request when it is used together with a time intensifier (i.e. 'COB' and date) and this request is quite direct and forceful. In this email, Megan, the administrator, requests her colleagues to send her the names of employees recommended for training. By using 'please' in conjunction with a boosting device, Megan is telling her colleagues to comply with her request. In short, the use of 'please' in conjunction with a mitigating device has the effect of softening the illocutionary force of a request but with the presence of a boosting device, it has a converse effect.

Another interesting point to note about the use of 'please' is the position that it occurs in a mood clause. Stubbs (1983) claims that 'please' can co-occur with all mood choices: imperative, interrogative, declarative and non-clausal unit, as shown in the examples below.

I'd like some more pudding, please. (declarative)

Can I have some more pudding, please? (interrogative)

Give me some more pudding please. (imperative)

More pudding, please. (moodless, truncated clause)

(Stubbs, 1983, p. 72)

And 'please' can occur in the initial, middle or final position in a mood clause. To date, only two studies investigate the placement of 'please' in great detail. The first study, Green (1975), looks at the placement of 'please' on the four speech acts: demands, orders, pleas 
and requests. According to her, 'please' takes the utterance-final in requests, and occurs at the utterance-initial or final position in pleas but does not occur at the utterance-initial or utterance-final in both demands and orders. The second study, Sato (2008), investigates the degrees of politeness in American English and New Zealand English expressed by 'please' in three positions: initial, medial and final. According to him, the initial position of 'please' is associated with the directive acts of demands and pleas, the medial position of 'please' can be identified with conventional polite requests while 'please' in the final position is reserved for task based requests.

Some researchers have argued that a different position of 'please' could also indicate a different illocutionary force (e.g. Alsree, 1997; Sato, 2008). Sato's (2008) study shows that the directive force of 'please' is greater when it takes the initial position, where the speaker is more assertive, less compromising and unwilling to negotiate. Alsree (1997, p. 169), on the other hand, argues that 'please' in the final position "sounds more stern and peremptory" as it suggests an "afterthought". The following two examples from Company NZ1 which are realised in the interrogative mood appear to contrast Alsree's (1997) findings.

1. [peer to peer]

Could you send a new frame to [name], [company's name] and [company's name] please?

2. [peer to peer] can you also tell [name] about [name] please?

The two examples seem to suggest that the use of 'please' in the final position functioned as a bid for cooperation rather than a peremptory command. In the first example, the use of 'please' from peer to peer functions as a bid for Angela's cooperation because the task requested was not her (Angela's) responsibility and might cause inconvenience. Similarly, in the second example, using 'please' suggests that Susan is trying to gain Sally's cooperation, as it is a face-saving device, to pass on a message to a client on her behalf.

In my dataset, each occurrence of 'please' in a request clause was categorised according to the mood choices: imperative, interrogative, declarative and non-clausal unit (e.g. 'Status please'), and its position noted. 
'Please' occurred in two positions - initial and final - in a request realised in the imperative mood as the following examples show:

1. Please take off [name of programme] registration form and link it to monkey survey. Please let me know if you have any questions. (NZ1) (initial position)

2. Pass me a copy of the QP please. (M) (final position)

'Please' in interrogatives occurred only in the middle and final position in my data. Consider the following:

1. Could someone please ring [name of client] from [place]on [contact number] about helping him answer questions about [name of programme]. (NZ1) (middle position)

2. Can I have the original please? (NZ2) (final position)

3. Do you mind following up please. (M) (final position)

In my data, 'please' in declaratives occurred only in the final position. Here are three examples:

1. That would be the point to check out please. (M)

2. I need the annual operational budget for 2011 please. (NZ1)

3. [Here are] A few more changes for checking please? (NZ1)

There is only one example where 'please' occurred in the final positions in a non-clausal unit.

1. Status please. (M) 
Table 7.5 shows the frequency of occurrences of 'please' in the various positions in requests realised in the imperative, interrogative, declarative moods and non-clausal units.

Table 7.5 Position of 'please' in mood clauses

\begin{tabular}{lccccccc}
\hline Position of 'please' (mood clause) & \multicolumn{2}{c}{ Company NZ1 } & \multicolumn{2}{c}{ Company NZ2 } & \multicolumn{2}{c}{ Company M } \\
\cline { 2 - 7 } & No & $\%$ & No & $\%$ & No & $\%$ \\
\hline Initial (imperative) & 29 & 43 & 82 & 69 & 172 & 92 \\
\hline Middle (interrogative) & 17 & 25 & 33 & 28 & 1 & 1 \\
\hline Final (interrogative) & 16 & 24 & 3 & 3 & 2 & 1 \\
\hline Final (imperative) & 1 & 1 & 1 & $>1$ & 7 & 4 \\
\hline Final (declarative) & 4 & 6 & 0 & 0 & 3 & 2 \\
\hline Final (non-clausal) & 0 & 0 & 0 & 0 & 1 & $>1$ \\
\hline Total 'please' & $\mathbf{6 7}$ & & $\mathbf{1 1 9}$ & & $\mathbf{1 8 6}$ & \\
\hline
\end{tabular}

In this section, I have demonstrated that 'please' can occur in different positions. And as argued earlier, if 'please' is used in conjunction with other softening devices, the illocutionary force is played down regardless of the position it occurs. However, if 'please' is used together with other boosting effects, the request tends to come across as a command regardless of the position it occurs.

In the next section, discussions of request emails authored by superiors to subordinates (downward), subordinates to superiors (upward) and peer to peer (laterally) are discussed.

\subsection{Request emails downward}

According to Holmes and Stubbe (2003b, p. 34), "people typically use explicit and direct forms when they hold a higher position in the institutional hierarchy than their addressee(s), and the addressee's obligations are clear". In this situation, it is the subordinates' obligation to comply with the request and these patterns have been observed in earlier research (ErvinTripp, 1976; Vine, 2001). However, superiors do not always use explicit and direct forms. They may draw on a variety of linguistic and pragmatic devices to soften the illocutionary force of the request and use a range of strategies to strengthen the illocutionary force of the request. The aim of this section is to explore how superiors in the three workplaces managed rapport and did power. 


\subsubsection{Company NZ1}

In this section, I explore the various linguistic and pragmatic strategies used by Andy, the CEO, from Company NZ1 when asking her subordinates to do something.

Consider the first example (Email 7.2) which is directed to Teresa, the web designer, requesting her to update the company's webpage.

\section{Email 7.2 (SA07) NZ1}

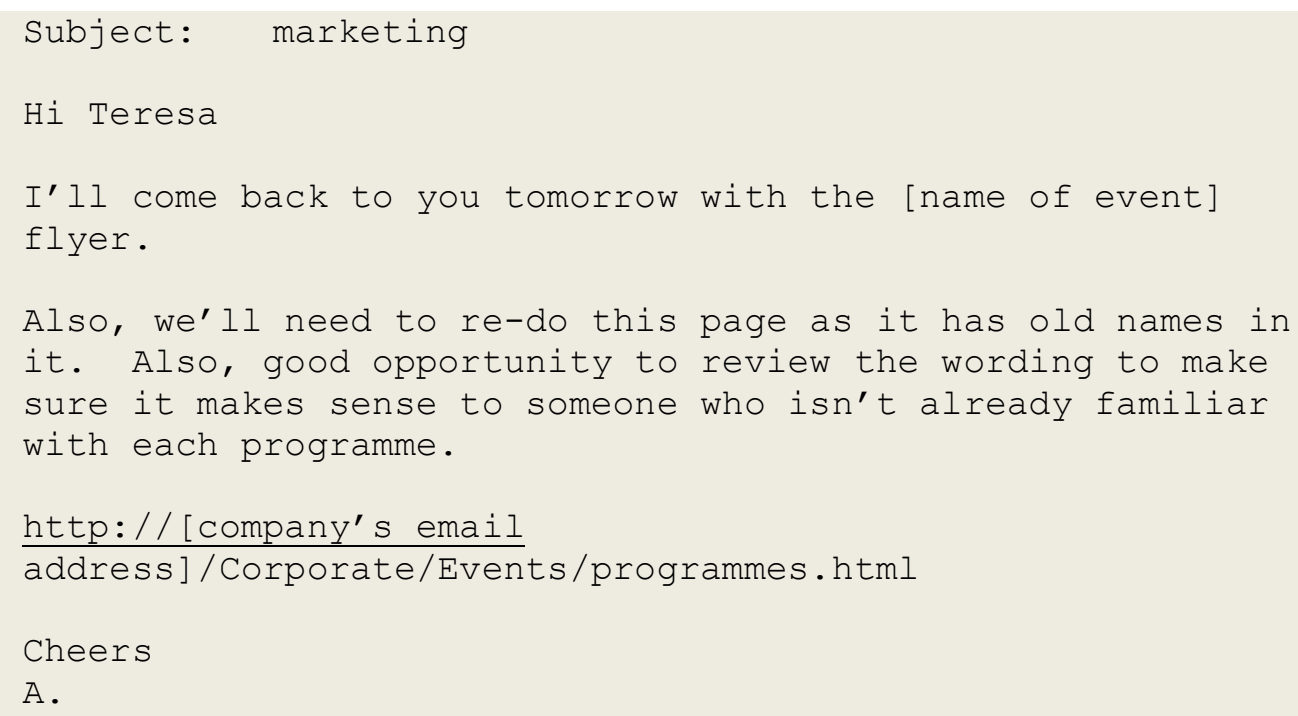

She begins her email with a greeting ' $\mathrm{hi}$ ' and addresses her subordinate, Teresa, by name. In this email, she makes two explicit requests: (1) to update the webpage by replacing the old names with the correct names (line 8); and (2) to review the wordings in the webpage so that it is easy to read and makes sense to lay people (line 9). She provides a grounder for each request. The first reason given is that the names on the webpage are old names and that they need to be replaced with the latest names. Her second reason is that it would make sense to people who have not heard of their programme (as in '... it makes sense to someone ...' in line 10). Lastly, she signs off the email with the formulaic 'cheers' and her initial.

Being asked to update a page could be regarded as face-threatening as it may suggest Teresa's incompetence at performing her job. Teresa, in principle, could be expected to have noticed the old names and does not need to be told that the web-page needs updating. By using three strategies, Andy attends to Teresa's face sensitivities and equity rights (Spencer- 
Oatey, 2005b). First, by providing a grounder, Andy minimizes the threat to Teresa's quality face (her competence and ability in web design) and identity face (her role as the web designer in the company). Providing reasons for her requests function as a mechanism to maintain rapport with her subordinate. In principle, as a CEO, Andy does not need to provide any reasons for the requests that she makes, and moreover, this is a routine task. However, giving reasons for the need to update the webpage could suggest that Andy is not overly concerned with status, a typical New Zealand attitude (Ashkanasy, Trevor-Roberts, \& Kennedy, 2004; Bönisch-Brednich, 2008; Holmes, Marra, \& Vine, 2012; Trevor-Roberts, Ashkanasy, \& Kennedy, 2003). On the other hand, the request could also be interpreted as a subtle way of telling Teresa that she should have noticed the old names without being told.

Second, the first request is made in declarative mood ('we'll need to re-do ...' in line 8) which is the least face-threatening form of a request. Using declarative mood may reduce the forceful and direct nature of telling the subordinate that she has made a mistake. The second request is ellipted ('good opportunity to review ...' in line 9) and suggests that Andy avoids naming the person who should do the reviewing task. Moreover, by attributing it as 'good opportunity', Andy seems to suggest that it would be helpful to carry out the task which has to be done sooner or later.

The third strategy, by framing the request with the pronoun 'we', the task of re-doing seems to be a shared burden. Andy could have framed her request as 'You need to redo this page ...' (line 8); instead she uses the pronoun ' $w e^{\prime}$ ', which signals solidarity, to emphasize team work as well as to suggest that they will be doing the requested task together. File (2013) who investigates discourse in sports interviews states that the pronoun 'we' is a useful strategy to construct team effort. Using the pronoun 'we' in this email could indicate that Andy is attending to Teresa's association rights.

In this email, by including an informal greeting and closing, Andy could be enhancing rapport with Teresa by attending to the 'involvement' component of Spencer-Oatey's (2005a, p. 100) interactional principles, i.e. association principle.

While Andy attempts to build rapport and do collegiality with her subordinate in Email 7.2, the imperatives in the Email 7.3 indicate that the power relationship is clear and uncontested. This email is an example which supports Andy's view which she expressed in an 
interview that email is excellent for sending information to a number of people so that everyone gets the same message at the same time.

\section{Email 7.3 (SA65) NZ1}

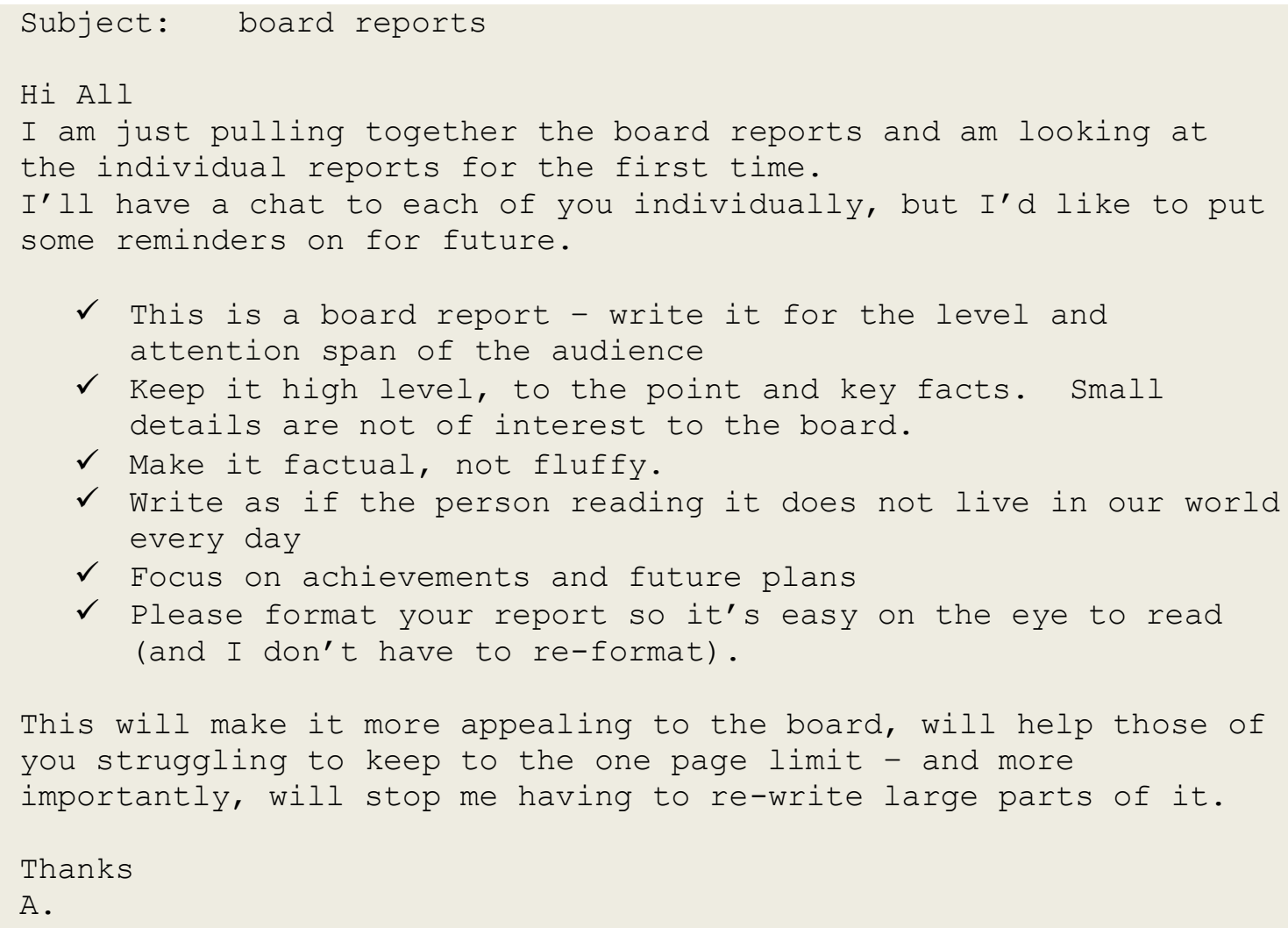

Andy begins her email with a greeting 'hi' and addresses all the HOEs and managers 'All' as she usually does when writing to more than one staff members. After initial friendly opening sentences (lines 4-7), she uses a string of imperatives, most of which are bare and unmitigated to tell her subordinates how she wants the reports to be presented. She signs off with 'thanks' followed by the initial of her name.

In this email, Andy uses six bald-on record imperatives 'write it for the level ...' (line 9), '... keep it high level ...' (line 11), 'make it factual ...' (line13), 'write as if ...' (line 14), 'focus on ...' (line 16) and '... format your report ...' (line 17). According to Ervin-Tripp (1976), when the task asked is generally expected to be the addressee's responsibility, unmitigated requests are expected. In this situation, it can be assumed that the recipients who are managers are expected to write good reports. 'Please' in line 17 does not seem to have the effect of 
reducing the force of the request, but instead, it boosts the illocutionary force of the request since it follows a list of imperatives.

In addition, the repetition of the importance of a concise report in line 18 ('I don't have to re-format) aims at getting the team to comply with her request. It is also clear that Andy's use of the material process (realised through the use of verbs such as 'write', 'keep', 'make', 'focus' and 'format') in the email which functions to help Andy construe the desired physical actions, suggests that importance is placed on getting the job done rather than on maintaining rapport with her subordinates.

Lines 20 to 22 appear to play down the illocutionary force of her request because Andy frames it as a benefit to her subordinates: first, they just need to produce a one page report; and second, Andy does not have to re-write their reports. However, despite framing the request as a benefit and providing three logical reasons in lines $12,14-15$ and $17-18$, it is obvious that Andy exerts her authority and power in this email. Signing off with 'thanks' (or 'cheers', which she uses in some other emails) and her initial does little here to attenuate or decrease the force of her request. This email clearly illustrates Andy's assertion of power. In addition to the use of imperative, the use of the personal pronoun 'I' coupled with material processes can also indicate power and authority. Consider the following example (Email 7.4). 


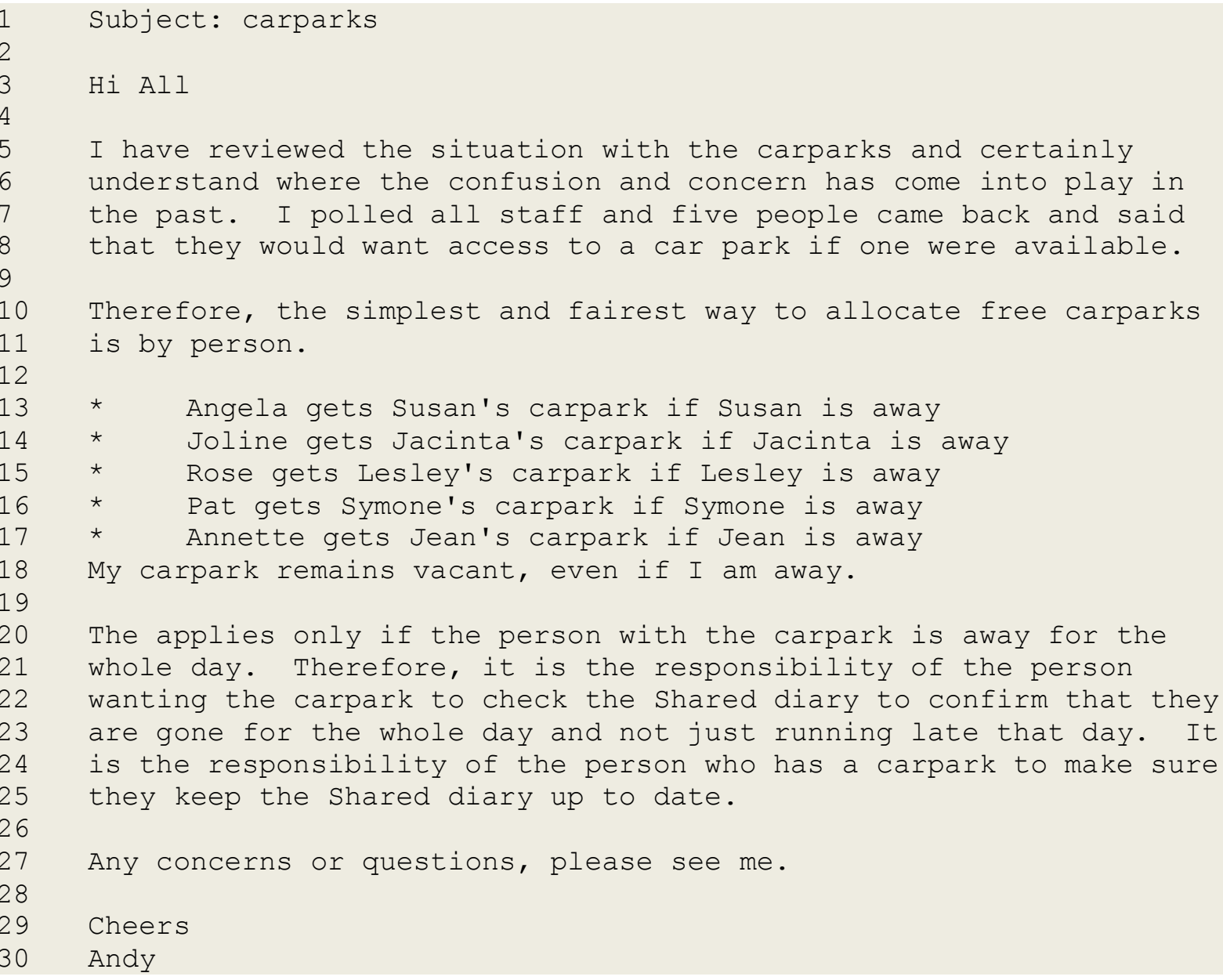

Andy, the CEO, sent this email to all staff members to inform them of the solution to a car park problem which once caused an email war among a few staff members. In this email, after her usual greeting 'Hi All' to address all staff, she uses the personal pronoun ' $\mathrm{l}$ ' and material processes such as 'I have reviewed ...' (line 5) and 'I polled ...' (line 7) to instruct the staff members to abide by her decision. By conveying her request through material processes rather than other processes such as 'I think' or 'I feel', she makes her actions explicit. The choice of the pronoun ' $I$ ' is used to construe actions that are personal to the writer; thus this indicates that Andy is in control of the situation. According to Holmes, Marra and Vine (2011) who investigate how Pakeha and Maori do leadership, Pakeha leaders tended to use the first person pronoun ' $I$ ' to construct themselves as successful leaders.

In line 27, however, Andy could be seen as attending to her staff members' association rights when she constructs herself as being an understanding superior by offering her staff the 
option to discuss with her if they have any concerns. And rapport is once again established when she ends her email by signing off 'Cheers' followed by her name. But despite evidence that Andy may be managing rapport with her staff, this email illustrates clearly that she is in charge and she expects her power to be uncontested.

We have seen in this section how Andy uses various strategies to: (1) do rapport with her subordinates (e.g. inclusive pronoun 'we', grounders, declarative mood, greeting and closing); and (2) assert her power and authority (e.g. pronoun ' I', bald-on imperatives, repetition and material processes).

Next I turn to a discussion of email requests in Company NZ2.

\subsubsection{Company NZ2}

In this section, I show how two superiors (Sheila and Patrick) from Company NZ2 do collegiality and do power in their emails.

Email 7.5 is written by Sheila to her subordinate, Ellen, and it is copied to her other subordinate, Selina.

Email 7.5 (SS05) NZ2

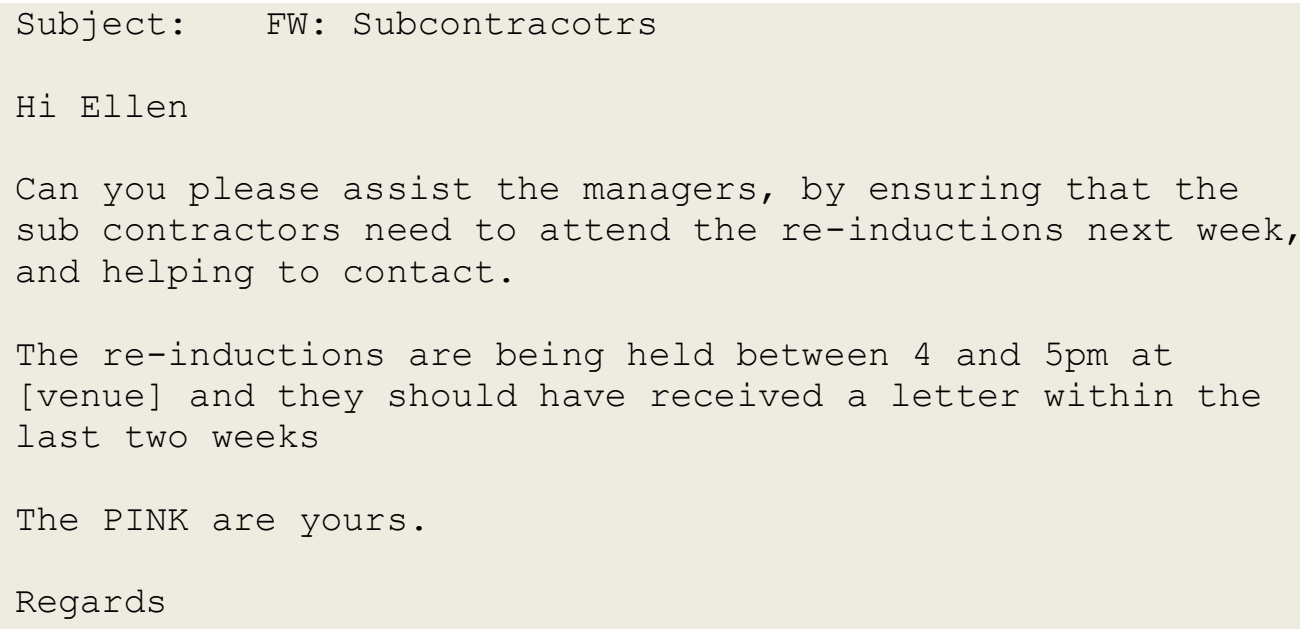

Sheila begins her email with a greeting ' $h i^{\prime}$ and addresses her subordinate, Ellen, by name. In line 5, she requests Ellen to assist the managers to contact all sub-contractors to ensure that they (sub-contractors) attend a re-induction training. She provides additional information of the training in line 9 but does not include any grounder for this request. In line 13, Sheila 
suddenly switches to a different matter, making reference to the invoices she returned to her (Ellen). Lastly, she signs off with 'regards' and though she usually includes her name when she signs off her emails, in this case she omits it.

The greeting 'hi' followed by the name of her recipient could be interpreted as doing solidarity and attending to the recipient's association rights, i.e. the interactional association-dissociation (Spencer-Oatey, 2000a, p. 15). Sheila phrases her request using an interrogative clause 'Can you please ...?', which according to CCSARP (Blum-Kulka et al., 1989a), is considered a conventionally indirect request. According to Holmes and Stubbe (2003b), superiors use more imperatives when delivering directives for routine tasks and when the power relationship is clearly defined. In contrast, my analyses suggest that interrogatives are more frequently used by the participants in Company NZ2 than other forms in contexts where tasks were considered routine (see discussion in Section 7.3.2.2) (cf Holmes \& Stubbe, 2003b). The use of 'please' suggests this is a request rather than a question about Ellen's ability to do the task requested. In other words, while 'please' may be intended as a politeness marker, in this email, it also intensifies the illocutionary force of the request (cf Brown \& Levinson, 1987).

Although her request is issued without providing a grounder and may be perceived as direct, it is accepted and considered a norm in this CofP (Wenger, 1998a). Moreover, when the requests made are routine and within the job scope, such requests are considered legitimate and do not violate the equity rights of the recipients. See Chapter 4.4 .3 for more discussion. The omission of a grounder when making the request also suggests that both Sheila and Ellen are familiar with each other as they have been working together for the past year, and thus Sheila can afford to dispense with politeness strategy (i.e. provision of a grounder). The spontaneous switch from one topic to another further illustrates the relaxed relationship between them.

Like the function of greeting, the use of closing serves to enhance rapport with the email recipients, as can be seen in this email when Sheila signs off with 'regards'.

The following email (7.6) is Patrick's reply his subordinate, Harry, who informs him of a serious problem that could potentially be very costly for their department. 


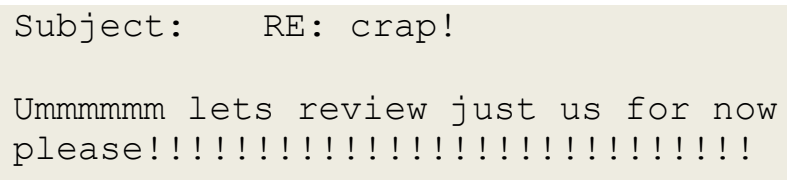

Patrick does not include a greeting or closing which is his typical style when replying to emails and launches straight into business, an observation that was confirmed by him in an interview. He reported that the omission of a greeting and closing was because he received a high volume of emails (about 200) daily. After the initial vocalised pause 'ummmmm' which could be interpreted as an expression to signal his thoughts or a gap before he 'speaks', Patrick frames his request as an imperative. The use of 'um' which is exaggerated in this email could also suggest that Patrick disagreed with Harry's opinion that the increase of cost in their department was caused by a wrong entry in the accounting system but the disagreement was done in a hedged manner. According to Wodak and Krzyżanowski (2008), the use of 'um' followed by a pause suggests that some form of disagreement is forthcoming and that complete agreement has not been reached. In addition, using the 'let' imperative with the modified pronoun 'just us', Patrick makes it clear that he wants only them to discuss the matter. The choice of the pronoun 'us' functions as a softening device and appeals to team solidarity. Using the material process 'review' that construes material action and evaluative meaning of finding a solution to the problem suggests that Patrick asserts control over the situation.

This email which is also copied to his superior, Albert, suggests that this problem is viewed seriously and needs to be resolved as soon as possible to avoid incurring further costs. As discussed in Section 7.3.3, the use of 'please' in the final position seems to suggest a bid for cooperation. However, in this email, the use of 'please' at the end of the request juxtaposed with the exaggerated number of exclamation marks (line 4) seems to intensify the force of the request that the matter should only be discussed among the three of them in the department. Overall, in this short email which consists of mixed strategies (e.g. no greeting and closing, pragmatic particle hedges, material process), Patrick has demonstrated a balance between asserting his power and authority and establishing a relaxed and close working relationship with Harry. 
The two examples analysed in this section have suggested a number of reasons (e.g. nature of request and relationship between interactants) why superiors in Company NZ2 often make direct and unmitigated requests when they want their subordinates to perform a task. I found this pattern repeated in a number of requests directed downward in my dataset.

The previous two sections looked at the different strategies superiors in the two New Zealand workplaces used when making requests downward. In the next section, I describe how superiors in the Malaysian workplace ask their subordinates to do something.

\subsubsection{Company M}

This section discusses the strategies that Amir, the CEO in Company M uses to do solidarity and assert power.

The following email (Email 7.7) is directed to three subordinates and the email is also copied to the Head of Department, who the three subordinates report to directly.

Email 7.7 (SA30) M

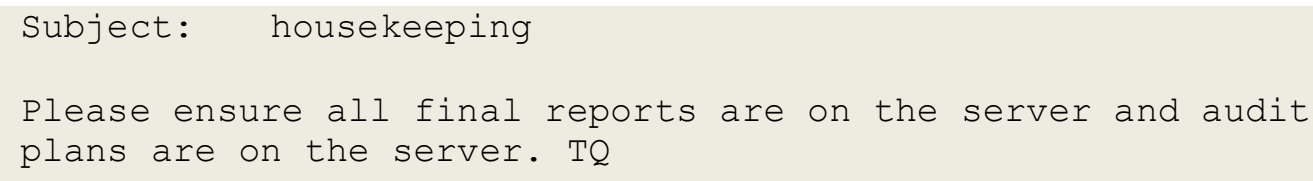

In just 17 words, Amir reminds his subordinates to make sure that they keep a copy of the final reports and audit plans in the company server. He does not include any greeting but ends the email with 'thanks' ('TQ' in line 4).

Amir's emails are usually short and direct, and this email is no exception. Amir's request is framed in the imperative mood (line 7). Here, my finding mirrors that of Holmes and Stubbe (2003b) who claim that the imperative form is often used by superiors when directives are concerned with routine tasks. The base form of the verb 'ensure' is realised as a material process and used with 'please' seems to suggest that it is an uncontested request. The choice of a material process to construe a physical action, in this case, to upload all final reports onto the server, clearly illustrates that Amir's instruction must be complied with. While it can be argued that 'please' is used as an attempt to mitigate the illocutionary force of a request, in this email, it appears to intensify the force of the request (see Section 7.3.3 for discussion of the use of 'please'). 
In this email, Amir seems to have violated Spencer-Oatey's (2000b) principles of attending to his subordinates' face sensitivities and sociality rights. According to Brown and Levinson $(1987$, p. 80$)$, more powerful speakers tend to be less polite than speakers who are less powerful. In this context, because Amir is the CEO and is at the highest rank in the organisational hierarchy, he seems to take advantage of this power (i.e. legitimate power (French \& Raven, 1968)) bestowed upon him by his workplace status to be less polite when issuing his request. By omitting the greeting and closing features, it could be interpreted that he might not be interested in building rapport with his subordinates. However, the inclusion of 'thanks' (i.e. TQ in line 8) as a closing expression suggests that he might be doing some minimal relational work.

Like Email 7.7, the following email from Amir is also short and direct, and it is directed to Uma, one of his subordinates. This email is copied to the HOD, who Uma reports to directly.

Email 7.8 (CA37b) M

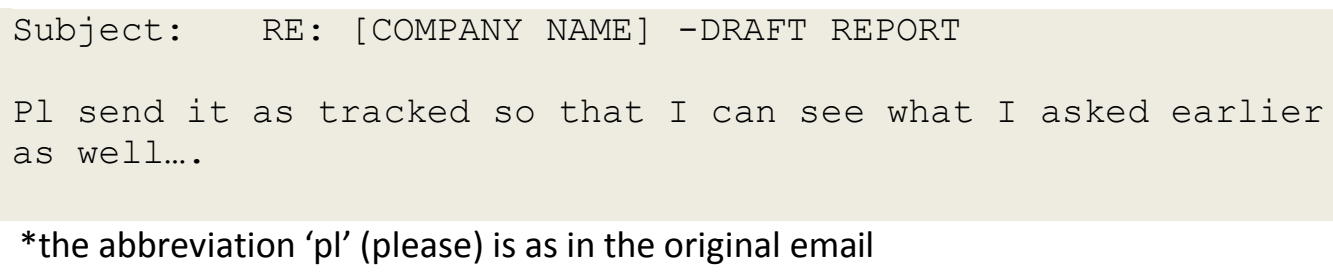

*the abbreviation 'pl' (please) is as in the original email

Like the previous example, this email does not have a greeting, Amir's typical style. Although Amir provides a reason why he wants a tracked version of the report Uma sent him, this is clearly a request. Providing a grounder here seems to suggest the urgency of the matter as well as a time saving strategy on Amir's part. As a CEO, he has many things which he needs to follow-up and the track changes in the document (i.e. report) would help him to address the issue quickly. Amir uses an explicit and direct form, bare imperative (line 3), realised as a material process which construes a physical action (i.e. send) in the clause. The use of the marker 'please' in the initial position seems to intensify the illocutionary force of the request. Not including a closing at the end of his email further indicates that Amir seems to challenge Spencer-Oatey's (2000c) principles of attending to his subordinate's face sensitivities and sociality rights.

In the next example, a longer email (Email 7.9) from Amir to two recipients - Chandran, the HOD and Kamal, a junior officer - he uses two types of mood clauses. 
Email 7.9 (SA41) M

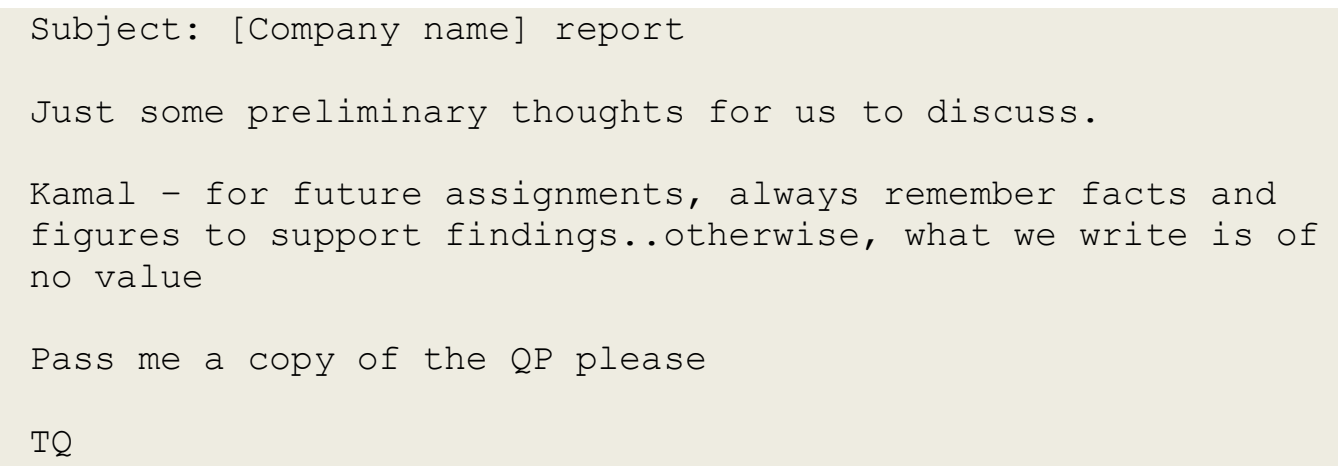

In this email, Amir does three things: (1) proposes a discussion; (2) reminds Kamal to include supporting evidence in the report that he (Kamal) prepares; and (3) requests a copy of a report from Kamal. He signs off with a formulaic and abbreviated version of 'thank you' (TQ).

In line 3 , his first request is directed to both recipients in the elliptical declarative mood. This message is softened in two ways: first, it is hedged with a phrase 'just some preliminary thoughts' to reduce the request force; and second, by focusing on 'us' rather than 'you' (line $3)$, it suggests team effort. Although this request is framed as an invitation, it is clearly a request which should not be contested. His second request, realised in the bare imperative 'remember', is addressed directly to Kamal. The illocutionary force of the request is increased with the presence of time intensifier 'always' in line 5. Unlike the first request which is attenuated, this second request is rather forceful. His third request, also realised in the bare imperative 'pass' in line 9 which construes a material action (i.e. give me a copy) strongly suggests that Kamal has to comply. Based on the context of the email, the presence of 'please' at the end of the request seems to suggest that this is a peremptory command rather than a bid for cooperation as argued in Section 7.3.3.

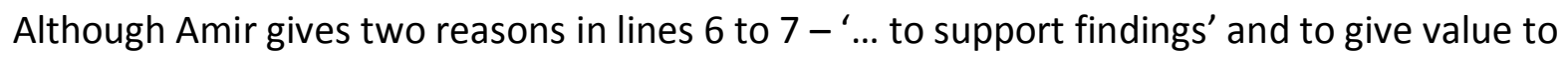
the report - to justify his request, the reasons seem to do little to attenuate the illocutionary force of the request. This email is clearly forceful with the presence of two bare imperatives (lines 5 and 9) and time intensifier 'always' (line 5). Hence, I argue that Amir appears to challenge Spencer-Oatey's (2000c) principles of attending to his subordinate's face sensitivities and sociality rights. 
In addition to using the imperative mood, Amir uses the exclusive pronoun 'we' and the second person pronoun 'you' to show power and authority, as can be seen in Emails 7.10 and 7.11.

\section{Email 7.10 (SA14) M}

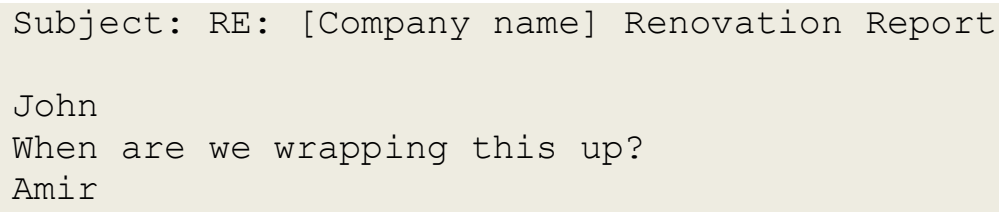

In this short email, Amir, the CEO, wants to know when his subordinate can complete the report for the company's client. Although Amir uses the pronoun 'we', it is obvious that John has the responsibility of completing the report for the client that he is put in charge of, a practice observed in this CofP (cf Ladegaard, 2011). According to Ladegaard (2011), the use of inclusive 'we' pronoun functions as a softening device, but in this email, Amir uses the exclusive pronoun 'we' (i.e. 'you') which indicates that John has the responsibility to revise the report.

Addressing the recipient by name alone and signing off with just his name further indicate that work takes precedence over social niceties. By using the Wh-Question, it suggests that Amir wants a specific date (for the completion of the report). This email is one example of Amir 'doing' power and challenging Spencer-Oatey's (2000c) principle of attending to his subordinate's face sensitivities.

In the last example, Email 7.11, Amir requests two of his subordinates to work together to produce an article for the company's internal newsletter.

Email 7.11 (SA13) M

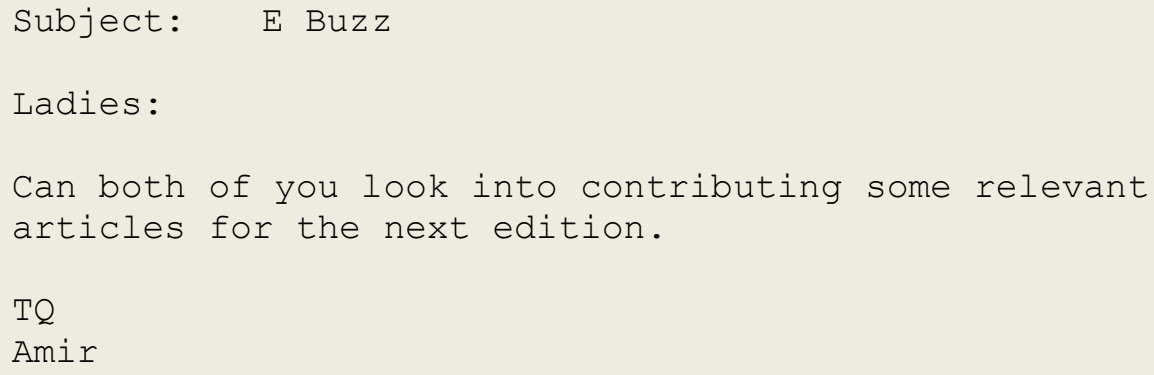


After addressing his recipients formally as 'ladies'(McConnell-Ginet, 2003), he uses the pronoun 'you' rather than 'we' to state clearly that he wants his subordinates to write an article (line 5). My observation field notes indicate that Amir used the address form 'ladies' interchangeably with the names of the addressees. This observation was confirmed in an interview with him. Signing off 'thank you' (TQ in line 8) with his name seems to have little effect to soften the illocutionary force of the request. The choice of the pronoun 'you' as well as the unmitigated style of giving request in this email clearly suggests that the CEO focuses on power and authority, a finding that mirrors that of Ladegaard's (2011) study.

In the Malaysian workplace, we have seen how the CEO takes advantage of his legitimate power and uses direct strategies such as the use of imperatives and omission of a grounder when making requests and this clearly indicates assertion of power. Although there is evidence of some attempt of building rapport with his subordinates as shown in Emails 7.8 and 7.9, the analysis suggests that the CEO's power is uncontested and his subordinates must comply with his requests.

To sum up, I am not suggesting that superiors in the Malaysian workplace always accomplish requests by using the bare imperative form. Rather I am suggesting that a preference for this form when making requests is consistent with the observation reported in Section 4.5.

\subsubsection{Summary of request emails downward}

People at work try to achieve transactional objectives while maintaining solidarity and collegial relationships among team members. Although superiors typically have the authority and right to request subordinates to perform a task, the manner in which the requests are made shows how superiors manage interpersonal relations with their subordinates and do power.

In Company NZ1, when Andy, the CEO, makes requests of her subordinates, she employs various strategies to manage rapport. She provides grounders and frames her request in the declarative mood, both of which may help to minimize the threat to her subordinate's face. She also uses the inclusive pronoun 'we' which emphasizes team work and signals solidarity. While maintaining good interpersonal relationships at work is important, getting work done is equally important. Andy also asserts her authority as discussed in Emails 7.3 and 7.4 in 
Section 7.4.1. Using a string of imperatives and the personal pronoun ' $I$ ' coupled with material processes, her request is unmitigated and uncontested.

As shown in the examples in Sections 7.4.2 and 7.4.3, the superiors in Company NZ2 and Company M seem to have violated Spencer-Oatey's (2000c) principles of rapport building. In Company NZ2, requests which are typically realised in the interrogative form accompanied by the maker 'please' and the absence of a grounder seem to intensify the illocutionary force of the requests. Similarly, in Company $M$, requests are typically expressed in the imperative mood, and the absence of a grounder and the omission of a greeting suggest that the subordinates must comply with the requests.

Overall, the discussion above presents the different strategies used by superiors in three workplaces to do collegiality and assert power. For example, while the requests by the superior in the Malaysian workplace were explicitly expressed using the bare imperative form, the superiors in the two New Zealand workplaces used a range of syntactic forms to express their requests.

\subsection{Request emails upward}

Asking someone who is in a superior position to do something can be challenging, and "politeness considerations typically weigh more heavily" (Holmes \& Stubbe, 2003b, p. 44). According to some researchers, requests addressed upward are expected to be less direct, mitigated and more formal especially in asymmetrical situations (Biesenbach-Lucas, 2007; Chen, 2006; Hartford \& Bardovi-Harlig, 1996).

There are two types of situations in my dataset where subordinates make requests of their superior: (1) in some situations the work responsibilities of subordinates required them to give specific and explicit instructions to their superiors; and (2) in other situations subordinates made requests which were of personal benefit to them. In this section, I discuss how subordinates in the three workplaces managed rapport and the various strategies they employed with reference to these two types of situation in their request emails to their superiors. 


\subsubsection{Company NZ1}

Sometimes subordinates are required to give specific instructions to their superiors due to the demands of their work responsibilities. Email 7.12 is one example. In this email, Susan, the Relationship Manager from the Corporate Team, requests Andy to insert her signature in the grant application.

\section{Email 7.12 (SS38) NZ1}

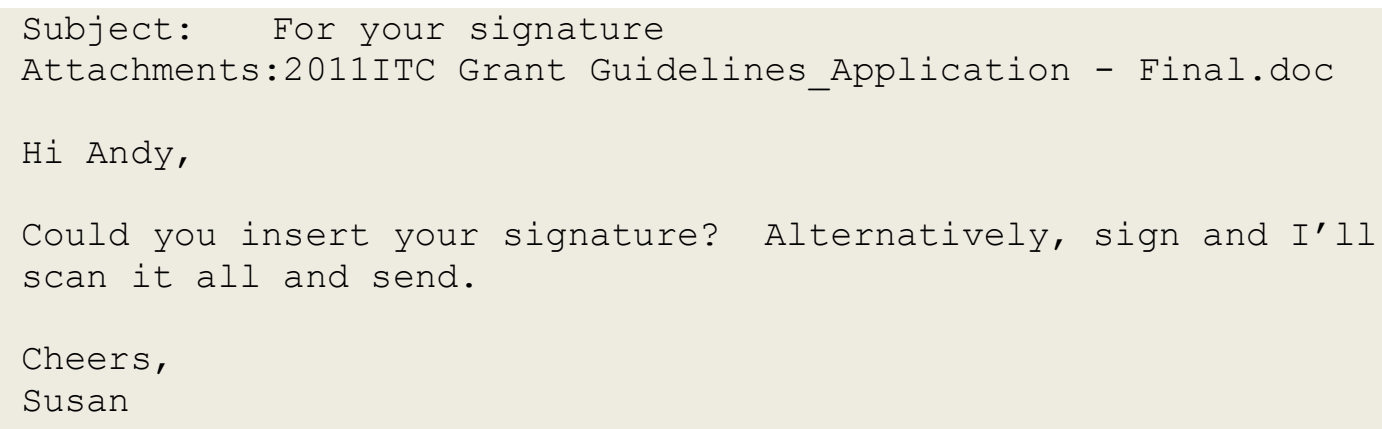

Susan usually begins her emails with a casual greeting 'hi' followed by the recipient's name (see Chapter 6). We see this pattern again here. Then she frames her request in the modalised interrogative form 'could you' rather than 'can you' as in 'could you insert your signature?' (line 6). Blum-Kulka, House and Kasper (1989a) show that 'could you' is less direct than 'can you' which in turn is less direct than the imperative form. Susan provides Andy with an option 'Alternatively, sign and I'll scan it all and send' (lines 6 and 7) which suggests that she is conscious that her request might threaten Andy's equity rights as her superior. It is clear that Susan is getting Andy to do something that is part of her job. Recognising the delicate situation of getting her boss to do her job, Susan skilfully offers Andy an option which downplays the force of her request and constructs the task as a minimal imposition on her (Andy). Signing off with her usual closing expression 'cheers' followed by her first name suggests that Susan is attempting solidarity and friendliness. In addition, she is also attending to her superior's face sensitivities (i.e. acknowledging her as her superior) and equity rights (i.e. not to be unduly imposed upon).

In Email 7.13a, Lesley requests permission to work from Auckland, and the first message from this chain email is an example of situation two, making a request for personal benefit. 


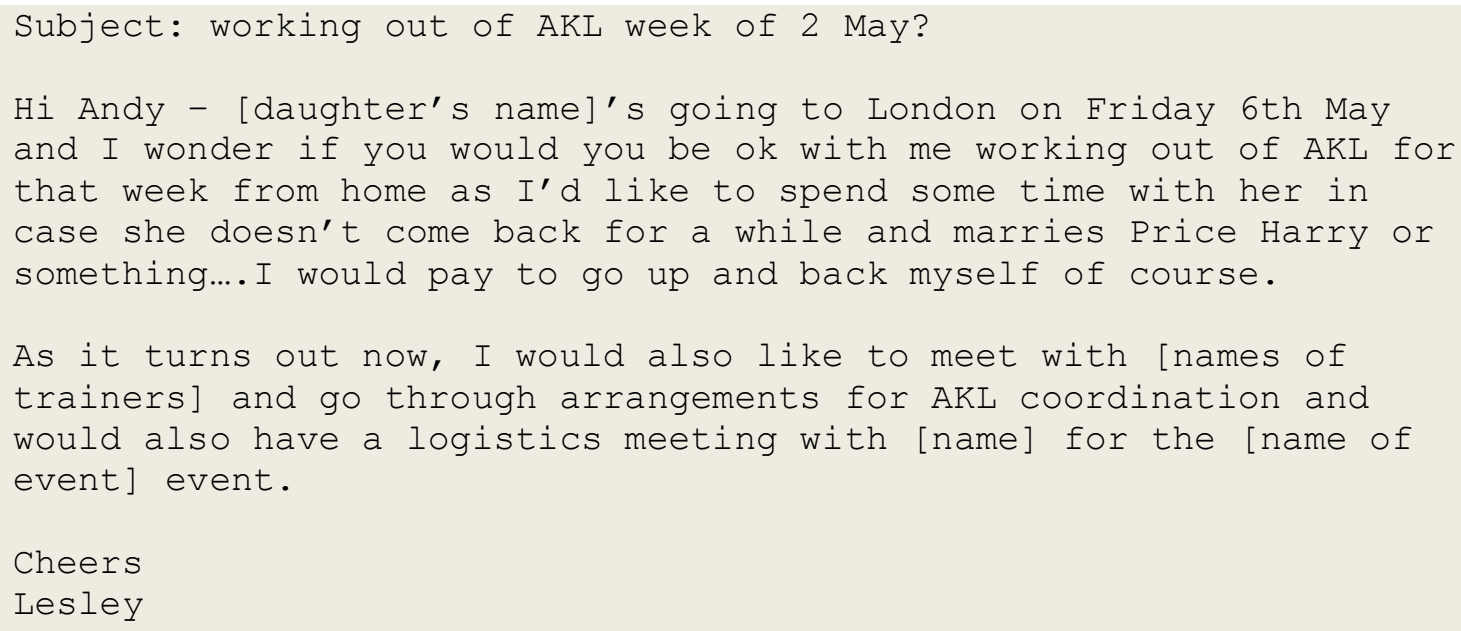

After her usual casual greeting 'hi' and acknowledging Andy by name, Lesley begins her email by informing Andy that her daughter will be going to London (line 3). This is followed immediately by her real agenda - request for permission to work from her home (line 4). When making this request of her superior, she uses a few mitigating strategies to soften the illocutionary force of her request. First, she used the mental process through the use of the verb 'wonder'; second, the modal 'would'; and third, the conditional 'if' to mitigate the illocutionary force of her request. By projecting it as a mental process, it suggests that Lesley is offering her personal view, thus, reducing the force of her special request. Using the pronoun ' $I$ ' with the mental process 'wonder'(line 3) helps Lesley to sound more tentative rather than assertive. She then justifies why she needs to spend time with her daughter. This justification is accompanied by humour as '... in case she doesn't come back for a while and marries Prince Harry or something ...' (lines 6 and 7) which suggests that she is constructing solidarity while making a request of her superior. In this organisation, as in most organisations, it is not a common practice to request permission to work from home. Because there is evidence that Lesley is doing more relational work (i.e. the use of humour and provision of grounders) here, this request is considered a high imposition request. Injecting humour into the request suggests that Lesley is attending to Andy's equity rights, i.e. of not being unduly imposed upon. In addition, humour is also a strategy used to release tension and for building solidarity (Holmes \& Stubbe, 2003b; Ladegaard, 2009; Schnurr, 2005; Schnurr \& Chan, 2011). She then continues to explain that she pays her own transportation costs as additional support for her request. 
In the following paragraph (lines 9 to 12), she informs Andy that she will be meeting the trainers and a client while she is in Auckland, which is work related. This explanation shows that Lesley is not taking advantage of the company while she attends to her personal agenda.

In this email to her superior, Lesley uses various linguistic strategies to attend to her superior's identity face (i.e. acknowledging her as her superior) and equity rights (i.e. not to be unduly imposed upon). The reply from Andy (email 7.13b) clearly shows that Lesley is successful in her attempt to do collegiality.

Email 7.13b (CA78b) NZ1

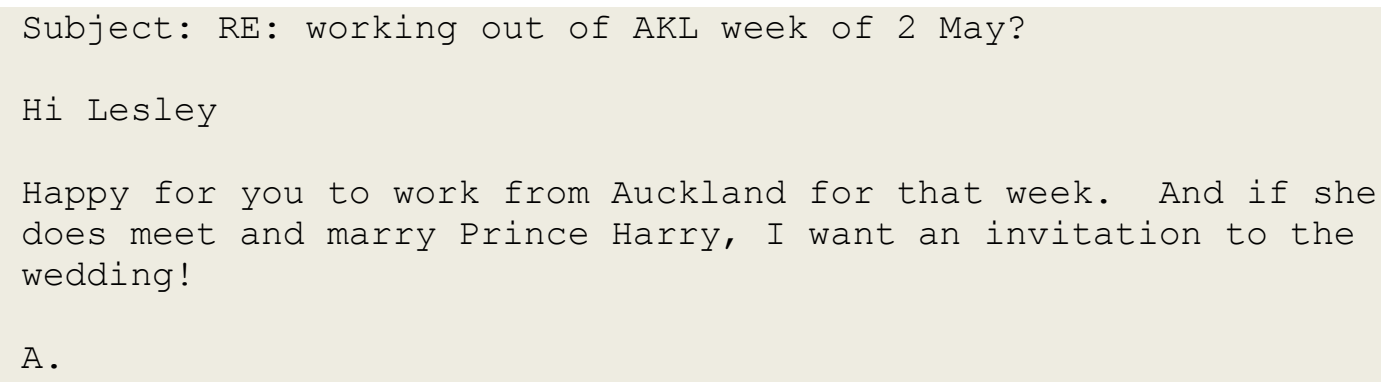

Andy not only grants Lesley approval to work from home (line 5) but she also endorses the idea of a royal marriage as evident in lines 6 and 7 ('if she does meet and marry Prince Harry, I want an invitation to the wedding!'). Both Andy and Lesley are clearly 'doing collegiality' as Lesley continues this chain of humorous sequence by sending Andy a picture of her daughter dressed to look both intelligent and sporty (see Email 7.13c).

\section{Email 7.13c (CL78c) NZ1}

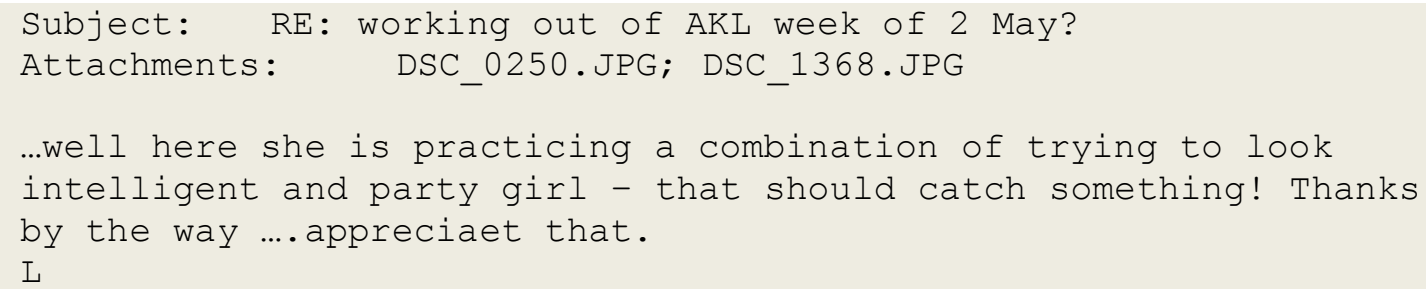

The email chain above clearly shows how the superior and subordinate share common ground by contributing to collaborative humour and develop "a humorous sequence for mutual amusement" while maintaining solidarity (Holmes \& Stubbe, 2003b, p. 111). 
In this section, I have presented a few strategies subordinates employed when making requests of their superior, Andy, which suggest that they are attending to Andy's face sensitivities and equity rights.

Next, I present a discussion of how subordinates in Company NZ2 make requests of their superiors.

\subsubsection{Company NZ2}

The first example of email upward is an email which satisfies situation one, i.e requests which are work related. In this email (Email 7.14), Ken requests his superior, Ron, the General Manager to approve a purchase order (PO).

Email 7.14 (SK70) NZ2

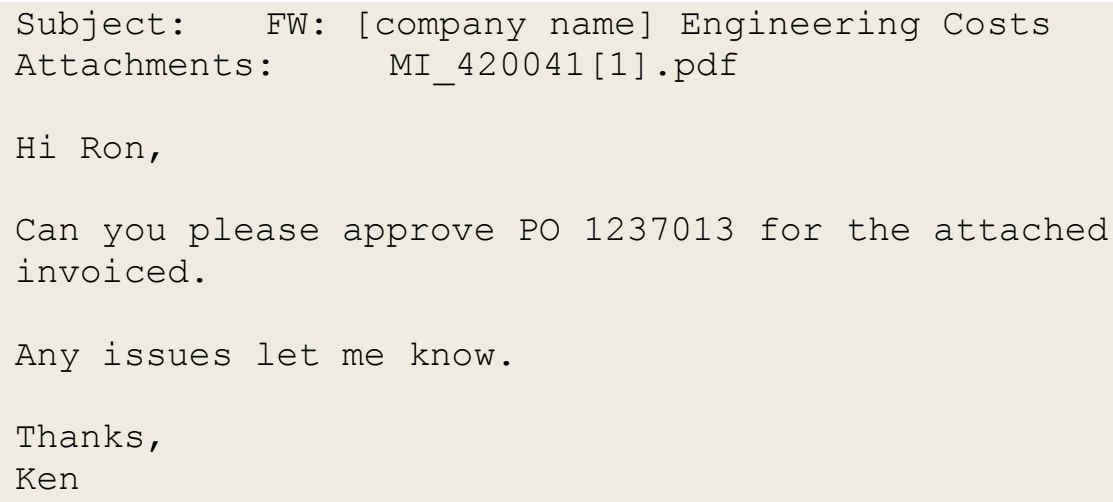

Ken greets his superior with his usual casual greeting 'hi' and acknowledges him by name. The informal greeting could be an index of the New Zealand cultural norm where the differences in status and rank are typically avoided (Bönisch-Brednich, 2008; Holmes et al., 2011, 2012; Trevor-Roberts et al., 2003). Although the request is work related, Ken frames the request to make it reasonable so that he is seen as attending to his superior's equity rights, and he does this by using a few strategies. His request is phrased in the modalised interrogative form 'can you', and the use of 'please' in this email is seen as a useful device to gain co-operation, as it is a face-saver because this request is made to the person with the highest authority in the hierarchy of the company. By offering assistance in line 9 'Any issues let me know', it can be interpreted that Ken is doing two things: first, it is his attempt to 
construct solidarity as well as attending to his superior's face sensitivities and equity rights because he 'instructs' his superior to do something (in this case to sign or approve a PO); secondly, he presents himself as a helpful subordinate who is ready to rectify any mistakes made. It could also signal that Ken is aware that there might be issues with the PO and by offering his help, he could be protecting his own face sensitivities: quality face (his competence as the Assistant Accounts Manager) and identity face (his role as a subordinate).

Finally, he concludes by signing off 'thanks', a formulaic expression of gratitude towards his recipient in anticipation of meeting his request.

Another example of email upward comes from Patrick, the Manager - Engineer, to his superior, requesting permission to take the company car for personal use. Email 7.15 is an example of situation two, making a request for personal benefit.

Email 7.15 (SP05) NZ2

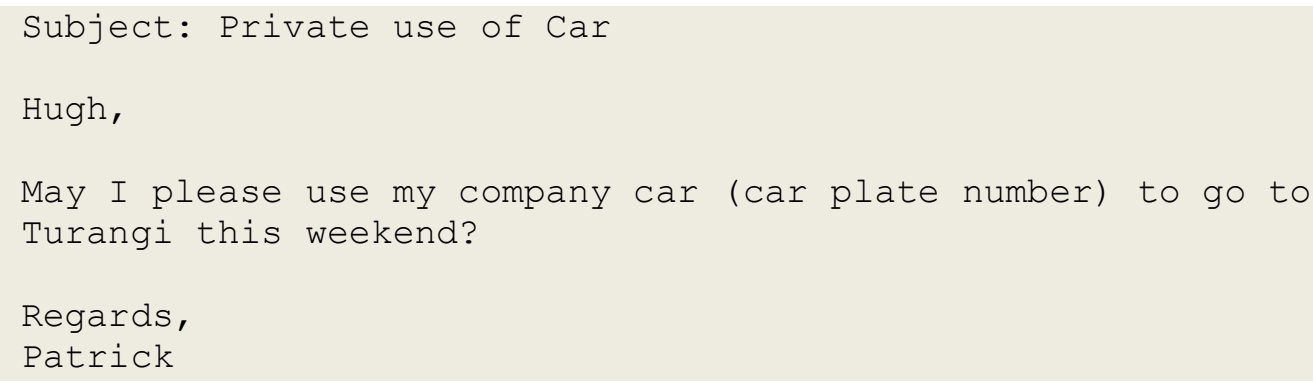

Unlike his typical emails which contain no greeting, he greets his superior by the first name. This is followed immediately by his request for permission to use the company car for his personal agenda for the weekend. His request is phrased in the interrogative mood with the modal 'may' which could be interpreted as a polite marker. The marker 'please' used here seems to further soften the force of the request as in 'May I please ...' (line 5). By doing this he "lowered the assertive force of his request" (Blum-Kulka et al., 1989a, p. 284).

This request is also accompanied by an explanation (grounder) of why he needs to use the company car for the weekend (as in 'to go to Turangi ...' in line 6). The use of various strategies to decrease the force of the request could also be interpreted as Patrick attending to his superior's equity rights, i.e. his rights of not being subjected to undue imposition. In addition, sending a short explanation and a clear subject line 'private use of car' (line 1) is to 
show consideration for his superior's time. By seeking permission to use the company car for his private use, Patrick attends to his superior's association right. Because Patrick is already in possession of the car, he could have used the car without informing his superior. In short, informing his superior of his intention could also be interpreted as a form of respect.

Finally signing off 'regards' followed by his name can be interpreted as a sign of solidarity and maintaining rapport as opposed to his usual style of not signing off his emails.

This email which is not typical of Patrick's style clearly shows that making requests which are considered out of the ordinary and unrelated to work requires special care and careful negotiation.

The next section examines the various strategies used by subordinates in Company $M$ when making requests of their superiors.

\subsubsection{Company M}

According to Lee (2011), respect for seniority and authority is considered very important in workplaces in Singapore and the same applies in Malaysia. In the Malaysian culture, it is considered impolite to address someone who is more senior or in high authority by first name. Watts (2005, p. 61) suggests that "socially appropriate behaviour is in fact politic behaviour and not polite behaviour". According to Watts (2003), politic behaviour refers to behaviour that is expected or appropriate in a specific type of interaction whereas polite behaviour refers to behaviour that is beyond what is expected in a specific type of interaction. If this is the case, then addressing people who are senior and in higher authority in workplaces in Malaysia is considered politic behaviour rather than polite behaviour.

Email 7.16 satisfies situation one referred to above, i.e. requests which are work related. Kamal requests his superior, Amir, the CEO, to check the audit report which he prepared for a client. 
Email 7.16 (SK15) M

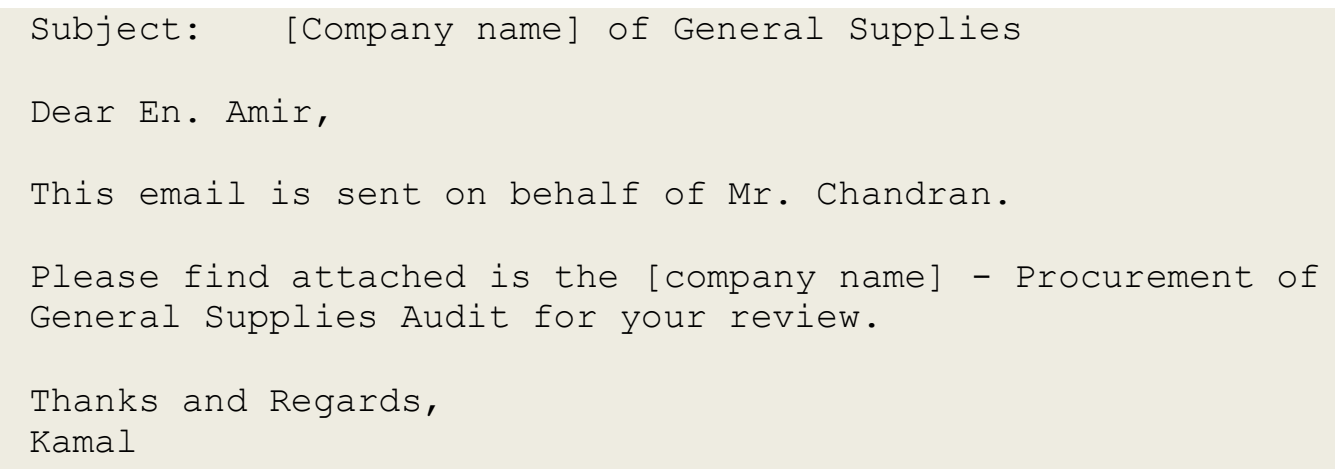

Kamal greets his superior with 'dear' followed by his title 'Encik' (Malay equivalent of Mr) and acknowledges him by name. He begins his email by informing his CEO that his direct superior (HOD) who he copies this email to is aware of the report he is sending: 'this email is sent on behalf of Mr. Chandran (line 5). This strategy could be interpreted as a way of protecting himself because the draft report must be approved by the HOD before the report is sent to the CEO, a protocol observed in this CofP. According to Chandran in an interview, he must "make sure that the facts are correct" before the report is sent to the CEO. Kamal's request is phrased in the imperative form using the material process which is realised through the use of the verb 'find' as in 'Please find attached ...' (line 7). Using the material process which construes the physical action 'read', Kamal is instructing his superior to read and check his report and this could challenge the equity rights of his superior. However, he minimises the imposition and appeals to authority by using 'please' in this email, which is seen as a politeness marker to soften his request. According to Economidou-Kogetsidis (2011) who conducts a perception study ${ }^{18}$ on emails which contained please + imperative, her participants did not consider these emails as having a negative effect. 'Please' in this email can also be interpreted as a face-saver and a form of respect because this request is made to the person with the highest authority in the hierarchy of the company. During an interview, Kamal said that he uses the imperative structure because 'that's the only way I know how to make a request' (quote taken from interview). In addition, he added that he is not very confident of his command in English.

\footnotetext{
${ }^{18}$ A perception study is a study where "participants were asked to offer their perceptions on the politeness and/or appropriateness of ... email messages"(Economidou-Kogetsidis, 2011, p. 3199).
} 
He concludes his email by signing off 'thanks and regards', a formulaic expression of gratitude towards his recipient in anticipation of meeting his request. The inclusion of a formal greeting 'dear' followed by the title and first name to address his superior and a closing expression shows that Kamal attends to his superior's face sensitivities and equity rights because he needs to 'instruct' his superior to do something (in this case to review a report which he prepared).

Email 7.17 is an example of situation two: making a request for personal benefit. In this email which is copied to the HR Manager and the CEO's secretary, Sharifah requests approval to go on leave from En Amir, her CEO.

Email 7.17 (SSO7) M

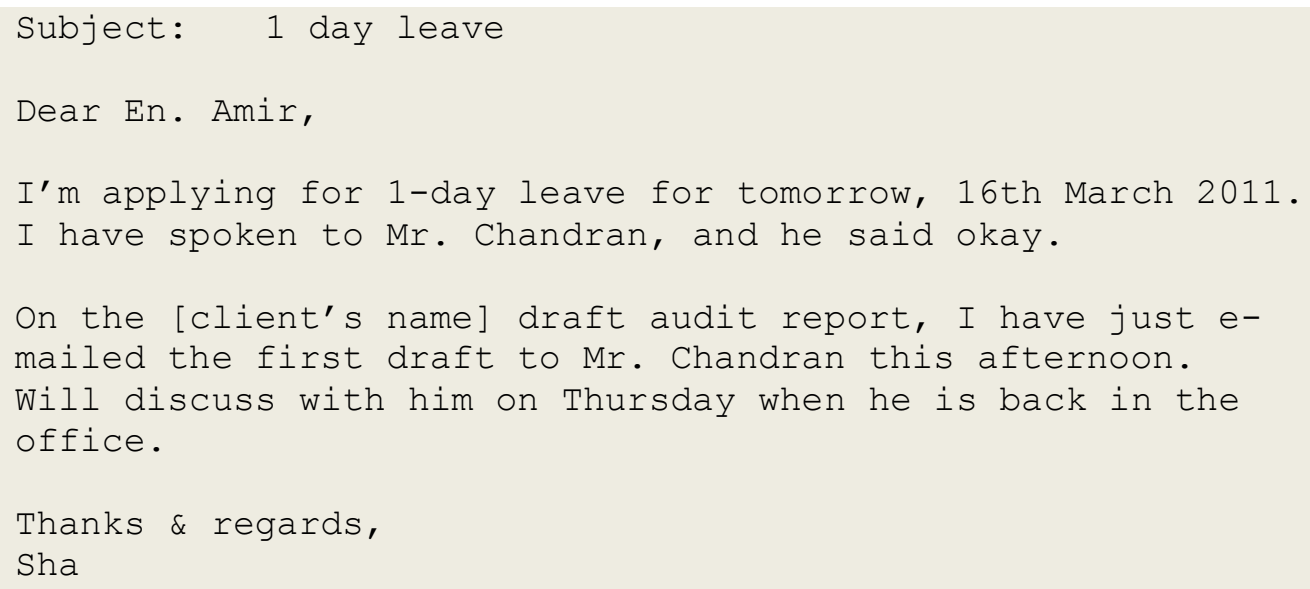

Sharifah begins her email by greeting her superior. She then informs her CEO, that her direct superior, Mr Chandran, has granted her permission to go on leave. Although she has obtained approval from her direct superior, informing her CEO that she is 'applying for 1-day leave for tomorrow' (line 5) could be interpreted as requesting for her CEO's permission and showing him respect. This request, which is projected as a material process (applying) construes the writer's physical action, and it is realised in the declarative form 'I'm applying for ...' (line 5), the least face-threatening form for making requests. The email ends with the formulaic sign off 'thanks and regards' followed by her name which is her typical style. Signing off emails is considered a sign of respect in this CofP as discussed in Section 6.5. The use of various strategies (the use of greeting and closing and the use of declarative mood to make her request) suggests that she attends to her superior's equity rights, i.e. his rights of not being subjected to undue imposition. 
We have seen how the subordinates in Company $\mathrm{M}$ relied on the use of formal greeting and closing in their request emails to their superiors. Unlike Company NZ1 where the subordinates used various mitigating strategies to soften the illocutionary force of their requests, subordinates in this workplace typically used the 'please' + imperative form, and did not provide grounders for their requests.

\subsubsection{Summary of request emails upward}

Making requests upward must be given careful attention, especially if the situation is not directly related to work responsibilities. The discussions above show that more linguistic and pragmatic strategies are used when making requests which involved non-routine tasks or special requests which are unrelated to work.

In Company NZ1, when making requests which are considered high imposition on their superior, staff members use more linguistic strategies (e.g. mitigating strategies such as humour, provision of reasons and an indirect form of requesting) to manage rapport as demonstrated in the emails above and many others in the dataset. Hence, the recipient's (in this case, Andy, the CEO) face sensitivities and sociality rights are attended to.

Similarly, subordinates in Company NZ2 use more linguistic strategies to manage rapport and do solidarity when making special requests upward. In the emails above, personal requests made are accompanied with a greeting and closing. Unlike their typical request emails which are direct, personal requests are supported by reasons, and when reasons are offered, they are usually short and to the point. This pattern emerges in many other emails which perform this function in the dataset.In addition to the inclusion of greeting and closing, one participant (Ken) constructs himself as a helpful subordinate by offering assistance if needed. Hence, the superior's face sensitivities and sociality rights are attended to.

In contrast, in Company $\mathrm{M}$, all requests upward are accompanied with a formal greeting and closing as a strategy to attend to the superior's face sensitivities and equity rights. Staff members do not usually provide grounders when making requests which are work related, and they tend to use the non-modalised imperative form. The high use of the imperative, consistent with what Waldvogel (2005) found in her study of language use on the factory 
floor and university office, was the unmarked code of choice, a shared repertoire, in Company M. On the other hand, requests of personal benefit are justified.

My finding in relation to its directness and lack of mitigating devices is consistent with studies conducted on email requests of native vs. non-native speakers of English. Hartford and Bardovi-Harlig's (1996) US study on L2 learners email communication with their professors shows that international students' emails were more lengthy, and these students used fewer mitigating strategies in their emails. As a result, these emails were perceived as impolite by their professors. Chen (2001) investigates the differences between American (NSs) and Taiwanese (NNSs) graduate students in their emails requests (appointment, special consideration, recommendation) to their professors and finds that the NNSs employed more 'want' statements and less lexico-grammatical modifications which resulted in their requests becoming more direct and impolite. Other similar studies conducted also find that NNSs' failure to use a combination of mitigating devices resulted in what Thomas (1995) labels as pragmatic failure (Biesenbach-Lucas, 2007; Chen, 2006; EconomidouKogetsidis, 2011). However, it is important to note that the directness employed in the request emails in Company $\mathrm{M}$ is not interpreted as impolite at the CofP level.

In sum, while the subordinates in one New Zealand workplace (Company NZ1) employed various strategies when making requests upward in their attempt to do collegiality, the subordinates in Company NZ2 and Company M relied on the use of greeting and closing. Company NZ2 and Company M differed in the type of greeting and closing used.

\subsection{Request emails laterally}

Requesting a colleague to do something when both are of equal or near equal status in the institutional hierarchy requires careful attention to avoid damage to work relationships. In the following paragraphs, I discuss how email authors in each of the three workplaces manage rapport and do power in their request emails to their colleagues who are of equal or near equal status. 


\subsubsection{Company NZ1}

Participants in this workplace employed various strategies when making requests of their colleagues who were of equal or near equal status. The use of linguistic resources, humour and provision of grounders are evident in the examples below.

Consider the following email (Email 7.18) from Lesley, one of the Heads of Events, making an appeal to her colleagues, other fellow managers and the support team, to contribute an article for the company's newsletter.

Email 7.18 (SL31) NZ1

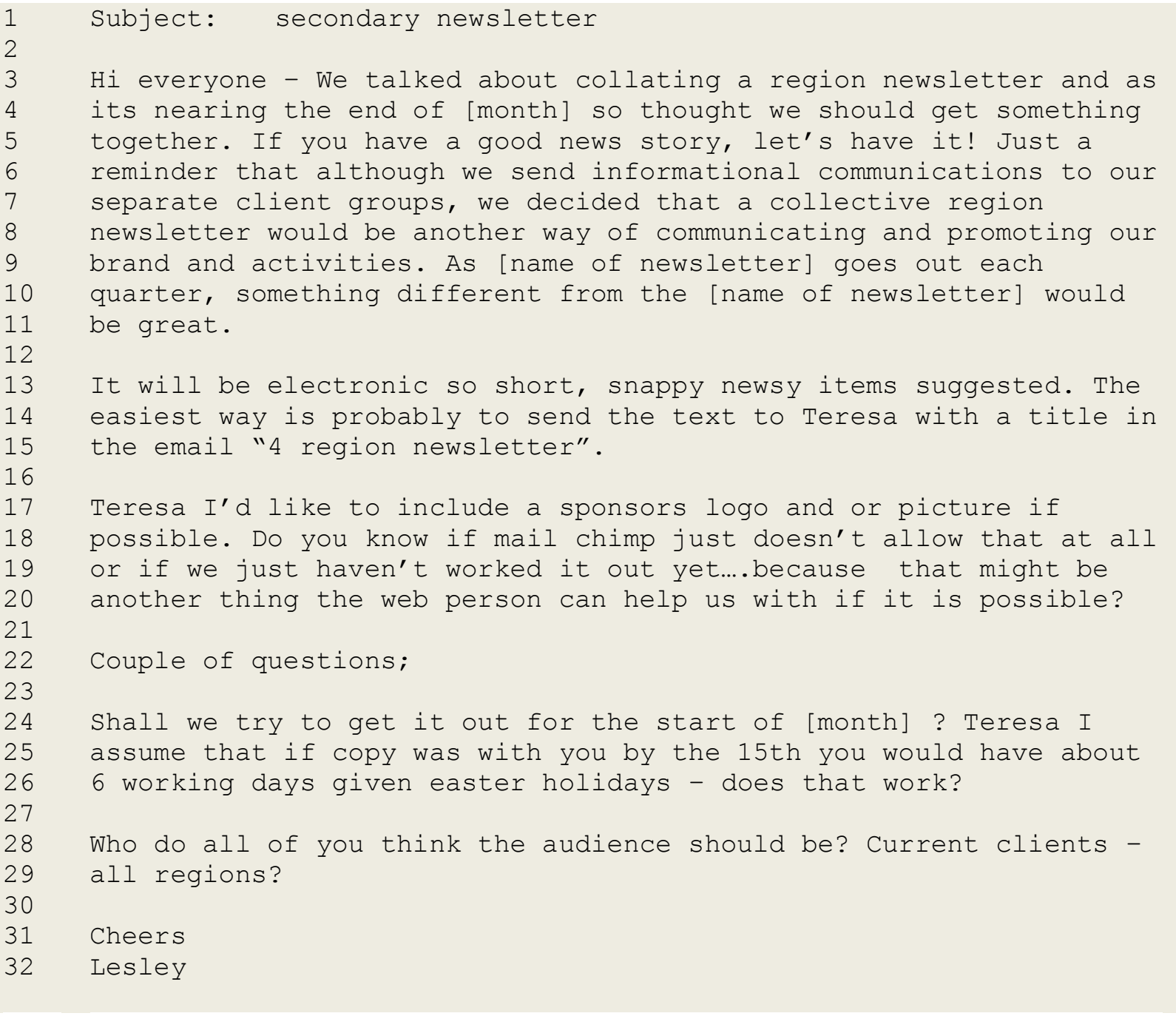

In this rather long email, Lesley makes two requests of her colleagues. Her first request is directed to all the HOEs to contribute to the proposed new online newsletter. Her second request is directed specifically to Teresa, the web designer, to work on the newsletter. 
Lesley employs several strategies in this email to get her colleagues to cooperate while maintaining rapport with them. She negotiates her request very carefully because what she has asked her colleagues to do is an additional task. She begins her email by greeting all her recipients 'Hi everyone' (line 6). She attends to the recipients' association rights in acknowledging that they have agreed to produce a collective newsletter instead of sending a separate newsletter to their clients as previously practised (lines 3-4 and 6-7). The choice of a particular pronoun will often determine the degree of cooperation. In this case, the use of the pronoun 'we' in line 3 'we talked' and line 7 'we decided' emphasizes that this is a joint decision, rather than simply her decision, a finding that mirrors other studies (File, 2013).

Instead of saying directly 'I want a good story from your event', Lesley uses three different means of mitigating the force of her request. She frames her request in a rather informal way 'If you have a good news story, let's have it!' (line 5) which suggests that the request functioned more as an invitation rather than an order. The use of an exclamation mark at the end of the request seems to signal a casual note and sounds almost like an encouragement to contribute to the newsletter. Using the conditional 'if' clause 'If you have ...' (line 4) to mask the task as a minimal imposition on them could be interpreted as giving her colleagues the option of contributing to the newsletter if they wish to. Hence, she attends to her colleagues' sense of equity rights (the right of not being subjected to undue imposition).

She uses words and phrases like 'short' (line 13), 'snappy news' (line 13) and 'easiest way' (line 14). The use of these words and phrases could be further interpreted as masking an additional task that could possibly take extra time and effort on the part of her colleagues. In short, these words and phrases play down the effort needed to perform the task. In addition, offering her colleagues the option of sending their story directly to Teresa (line 14) could mean that she gives them the impression that it is an easy task and that it will not take much of their time.

From lines 17 to 18 , she makes a direct request to Teresa realised in the declarative form as in 'I'd like to include ...' and hedged the request further with '... if possible.' She uses three different means of mitigating the force of her request. She uses the modal verb 'would' and the mental process through the use of the verb 'like' to soften her request rather than saying 'I want'. In addition, she gives Teresa the option of either using the sponsors' logo or picture. 
By employing these strategies, Lesley attends to Teresa's sense of equity rights (the right to be fairly treated and not unfairly ordered or taken advantage of).

In line 14, Lesley is taking the risk of violating Teresa's equity rights because she offers her colleagues the option of sending their stories to Teresa without first consulting her (Teresa). She appears to repair the blunder made which could potentially damage her working relationship with Teresa by consulting her and offers her room for negotiation as can be seen from lines 24 to 26 . Her skilful strategy is conveyed using a range of linguistic devices. She uses the pronoun ' $w e$ ' to signal team effort, the personal attribute 'I assume' in line 25 to put forward her suggestion in terms of timeline and checks with Teresa if she is happy with it (Halliday \& Matthiessen, 2004). The use of the personal attribute 'I assume' could also signal professional cautiousness where Lesley is careful not to impose the timeline on Teresa. By seeking Teresa's agreement as in 'does that work' (line 26) and including her in the decision making process as in 'shall we try to get it out ...' (line 24), Lesley attends to Teresa's equity rights.

To end her email, Lesley once again asks for the recipients' advice. By including all her colleagues in the decision making process (in this case the decision of who to send the newsletter to), she attends to their association rights (the rights to take part and be involved). In sum, she could be seen as acknowledging all her recipients again by addressing them first 'all of you' (line 28) before putting forward her request for their opinion in the interrogative form.

As with greeting, the inclusion of a closing serves to build rapport with her recipients when she signs off with her usual closing expression 'cheers' and her name.

Humour is one strategy of releasing tension, reaffirming group solidarity and "[maintaining] good relationships among colleagues, as a way of 'doing collegiality" (Holmes, 2000a; Holmes \& Marra, 2002; Holmes \& Stubbe, 2003b; Ladegaard, 2009, p. 207; Schnurr \& Chan, 2011). According to Holmes and Stubbe (2003b, p. 115), humour "expresses concern for the addressee's face and signals goodwill and positive cooperative intent". In one situation, Lesley uses humour to ask her colleague, Marcus, the Corporate Services Manager, to help her retrieve a folder which she has accidentally deleted from her email inbox as in Email 7.19. 


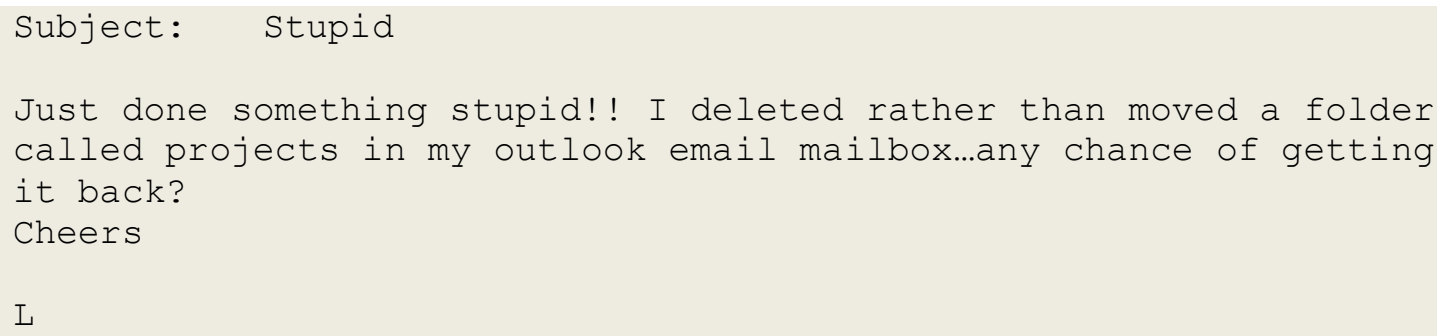

She begins her email with a humorous elliptical statement with two exclamation marks as in '[I] just done something stupid!!' (line 5). Although she knows that Marcus has the obligation to help her because he is in charge of any IT related issues in the company, she gives an explanation ('I deleted rather than moved a folder ...' in line 3) which is accompanied by a humorous statement. Providing Marcus with an explanation could be interpreted as reducing the imposition of her request.

Self-denigration (or self-deprecating humour (Holmes \& Stubbe, 2003b)), a key feature of Chinese politeness, is another strategy for attending to face sensitivities of recipients (Gu, 1990). Ho (2009) finds that this strategy was used by the Chinese members in his study in their attempt to mitigate the force of requests of peers to do something. By denigrating herself and acknowledging her incompetence, Lesley could be doing three things: (1) a plea to Marcus to help her; (2) a bid for his cooperation to prioritise this request because the deletion of a work folder can have serious consequences if it is not retrievable; and (3) to elevate Marcus' self-esteem by recognising his expertise. She frames her request in the ellipse interrogative form as in '[Is there] any change of getting it back?' further reduces the illocutionary force of her request because it gives Marcus an option of whether to help her or not.

In addition, Lesley chooses an interesting word (stupid) as the subject line. This simple subject line could be interpreted differently by different people but in this email, it seems to suggest that Lesley announces her own incompetence which probably could be viewed as recognising Marcus' expertise in IT matters. In other words, she attends to his quality face (i.e. his competence as the IT expert). It is not usual for a colleague in an organisation to admit his/ her own incompetence although accidentally deleting a folder is an easy error. In 
this email, Lesley could perhaps be exaggerating this act of deletion by phrasing it in a humorous tone (line 3 ) and reinforcing it with two exclamation marks.

In another situation, Susan frames her request in a humorous way, as illustrated in Email 7.20a. She has misplaced the batteries of her mouse and emails her colleagues to help locate them.

\section{Email 7.20a (CS19a) NZ1}

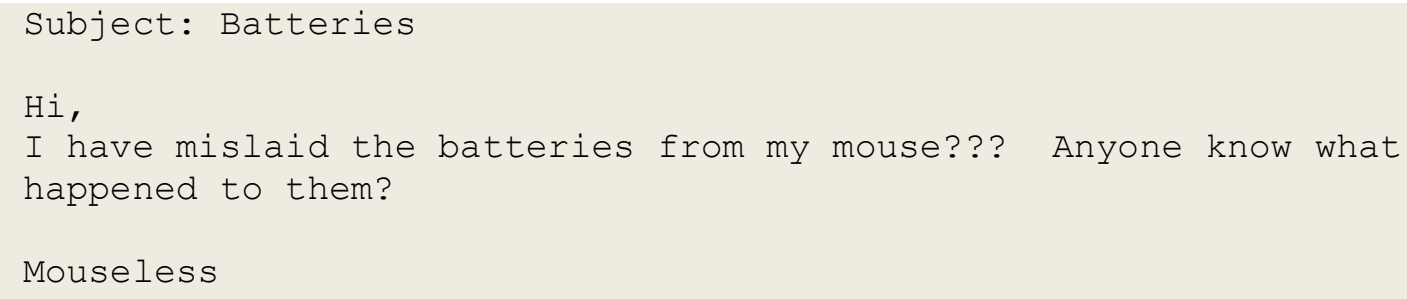

She begins her email with a greeting 'hi'. She announces that she has misplaced the batteries in the declarative mood and the presence of three question marks at the end of the sentence could suggest a sense of urgency. She then hints to her colleagues to help her locate them by asking them if 'anyone know what happened to them?' in lines 4 to 5 . By signing off as 'mouseless' (line 7), it appears that Susan attempts to soften her request as well as to amuse her colleagues. This injection of humour contributes to the construction of good relationship and solidarity between the colleagues, i.e. building rapport with the staff members.

It is also interesting to note that this humour was taken up further by Lesley as in Email $7.20 \mathrm{~b}$.

Email 7.20b (CL19b) NZ1

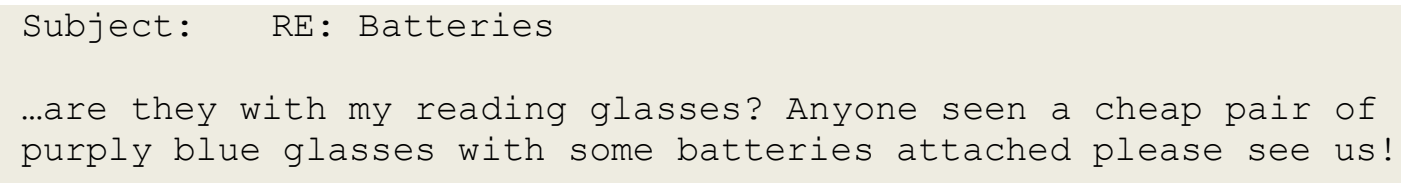

In Email 7.20b, Lesley who also coincidentally has misplaced her reading glasses plays along with the humour '...are they with my reading glasses?'. This can be interpreted as sharing common grounds and norms in the workplace. The sequence of "supportive humour" 
(Holmes \& Marra, 2002, p. 1687) shows Lesley's participation in the interaction. Managing humour is a sign of participation and doing solidarity.

Direct forms of request do occasionally occur in certain situations: when there is a recognised urgency, emergency or an unexpected due date. I make reference to two emails. In contrast to the two emails discussed above, Email 7.21 seems to display some form of power and shows very little consideration to attend to the recipient's face.

Email 7.21 (SS71) NZ1

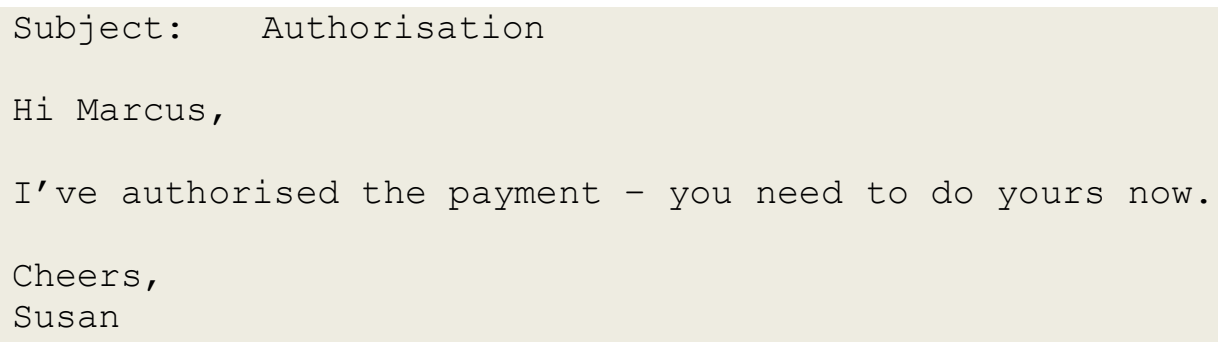

In Email 7.21, Susan, the Relationship Manager, informs Marcus, the Corporate Services Manager, that she has 'authorised the payment' and 'instructs' him to do his part, using the direct address form 'you' and declarative 'need' (line 5). She also incorporates the time intensifier 'now' to boost the strength of the request. She then signs off with her formulaic closing 'cheers' followed by her name. While the use of 'need' declarative and the direct address form 'you' clearly show the urgency of getting the payment processed, Susan also appears to be doing some rapport work by including an informal closing which could be interpreted as an attempt to re-establish solidarity and to attend to Marcus'equity rights (Spencer-Oatey, 2005b).

Consider the following situation, for instance, a chain email (Email 7.22) between Susan and Marcus. When Marcus appears to take offence at the direct form of request, Susan does some repair work.

In the first message of the chain email, Susan asks Marcus to check an application which she has prepared and requests some budget information. This email is sent to Marcus on his off day. 


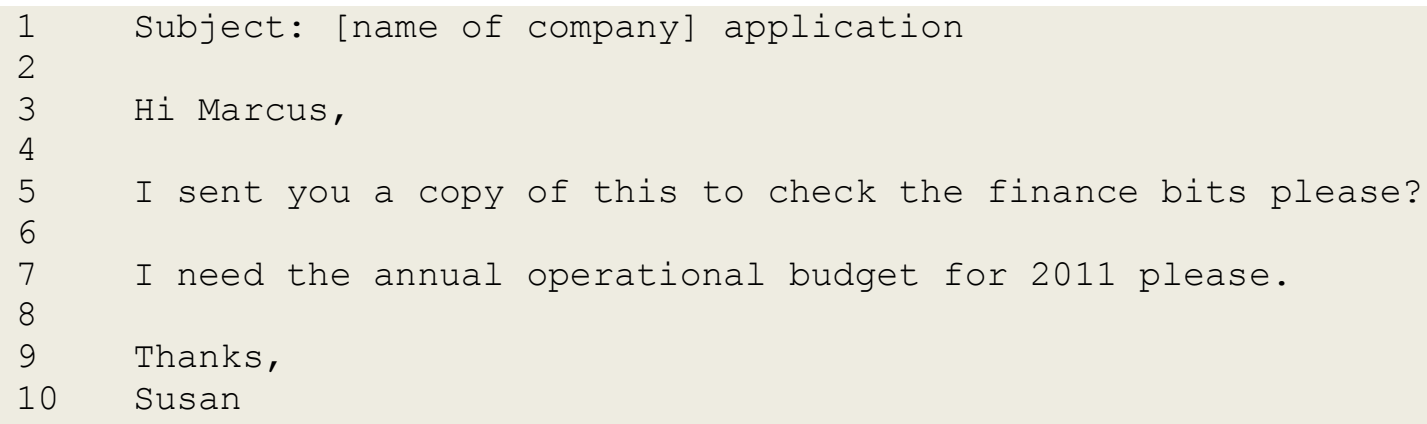

Susan makes two requests: (1) Marcus' help to check the finance section of an application which she has prepared (line 5); and (2) some budget information (line 7). The question mark at the end of the first request and the presence of the marker 'please' seem to weaken the strength of her request. However, the second request appears to be rather forceful. According to Holmes (1983) who cites Ervin-Tripp (1976), in a workplace setting, when a superior uses a need statement to make requests, it signals an obligation on the part of the subordinate. The illocutionary force shifts in the second request (line 7). The use of a need statement implies an obligation on the part of the recipient, and in this case, Marcus' obligation to comply. But the use of 'please' at the end of the request seems to balance the disequilibrium of getting a colleague of equal status to do something and functions as a bid for Marcus' cooperative behaviour.

The inclusion of a greeting ' $\mathrm{Hi}$ ' followed by name and the closing expression 'Thanks' and first name further re-establish solidarity in this rather forceful and direct request to a colleague of equal power and status. Besides using 'thanks' as a solidarity bid, it could also be Susan's attempt to do two things: first, it could signal her appreciation in anticipation of Marcus' compliance to the two rather assertive requests made; and two, it could be a way of showing her sincerity by not using her usual closing 'cheers'.

However, Marcus' reply (Email 7.22b) clearly shows that he is annoyed with the request because he is 'instructed' to do work on his off day.

\section{Email 7.22b (CM31b) NZ1}

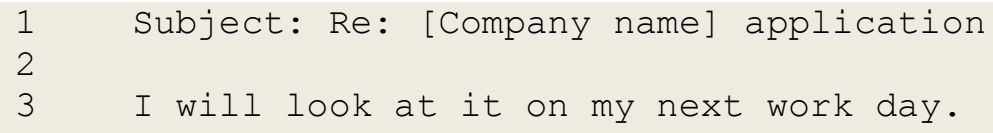


Marcus does not include a greeting or closing in his reply and informs Susan that he 'will look at it on my next work day'.

Susan picks up Marcus' annoyed tone from his reply and immediately responds to repair the damage done (Email 7.22c).

Email 7.22c (CS31c) NZ1

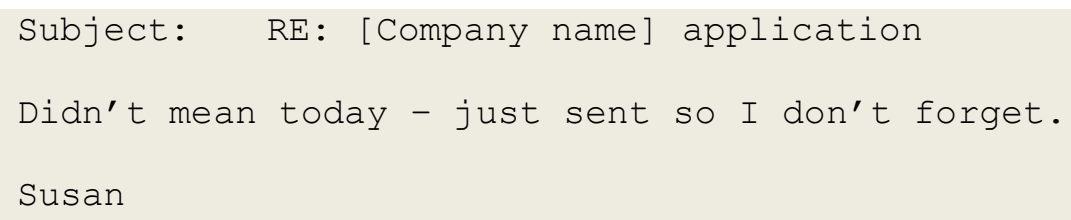

Sending an elliptical statement 'didn't mean today' and justifying why she sent the earlier email ('so I don't forget') shows that Susan appears to place an emphasis on maintaining rapport with the people she works together regularly while performing her transactional responsibilities.

The next email (Email 7.23) supports the argument made that Susan places an emphasis on maintaining rapport with her colleagues while performing transactional work.

Email 7.23 (SS60) NZ1

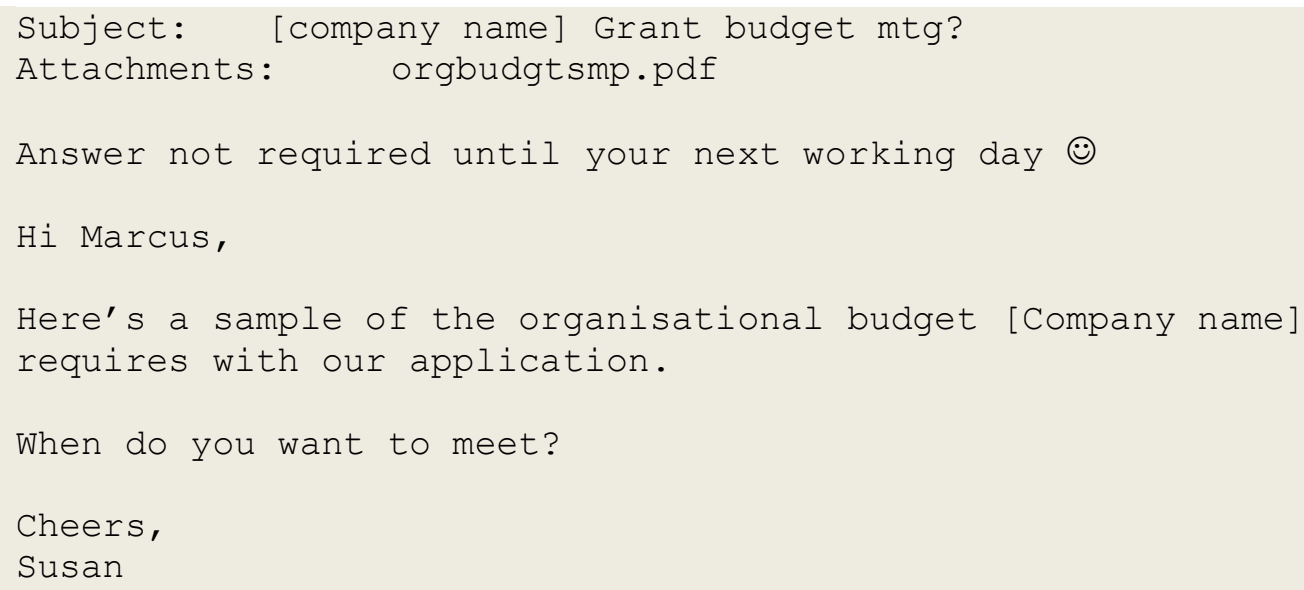

Email 7.23 is sent to Marcus on a Sunday, and to avoid any misinterpretation, Susan writes a caveat 'answer not required until your next working day' (line 4) to indicate that a response on his next working day would still be OK before launching into making her request. Writing the caveat could be interpreted as: (1) a sign of respect towards her colleague; (2) she avoids 
imposing on Marcus' freedom of action; and (3) she gives him the choice to response on his next working day. Inserting an emoticon, a smiley face, also functions as an effective strategy for reducing potential offence and constructing solidarity.

In sum, asking a colleague of equal or near equal status to do something involves some facework and this is apparent from a number of mitigating strategies discussed in the emails above. These email examples are typical of the many others found in my dataset, where equal or near equal status carefully manage their requests in order to maintain rapport and attend to each others' face sensitivities and equity rights (Spencer-Oatey, 2000c).

In the next section, I present a discussion of how peers in Company NZ2 make requests of each other.

\subsubsection{Company NZ2}

Unlike Company NZ1, participants in Company NZ2 were more direct when making requests of their peers. This directness supports Ho's (2009) findings and contrasts with Holmes and Stubbe's (2003b) findings. In Ho's (2009) study, the Chinese members preferred to use a direct request Head Act when making a request to their peers. In contrast, Holmes and Stubbe (2003b) finds that the use of direct requests was infrequent among peers in New Zealand.

Although participants in Company NZ2 show a preference for being direct when making requests of their peers of equal status or near equal status, there are differences observed in emails directed to individuals, a group of recipients, close colleagues and distant colleagues. In the following four examples, different strategies are used by the participants in Company NZ2 when making requests of peers of equal status or near equal status.

When writing to a group of recipients, some of whom are distant colleagues, a formal greeting is used. Staff members who were interviewed reported that formal greetings such as 'good morning' and 'good afternoon' were used when writing to peers who are at the managerial level as can be seen in Email 7.24. In the following example (Email 7.24) to a group of recipients, Sheila requests her colleagues to check and close any outstanding month end reports. 
Email 7.24 (SSO1) NZ2

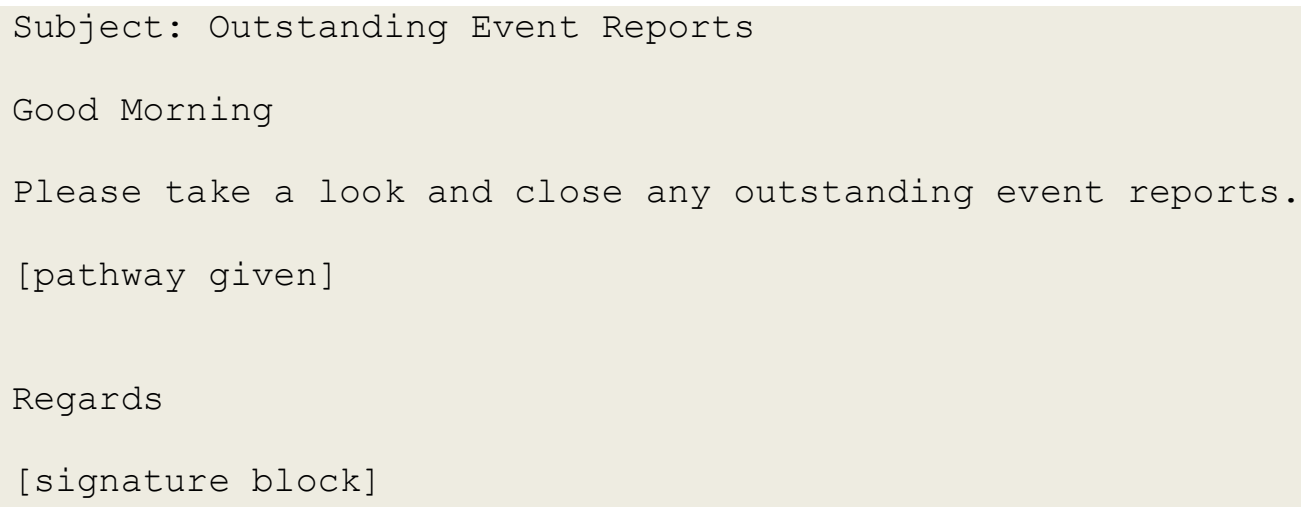

Sheila's email has a clear subject line. Staff members interviewed reported that a subject line is perceived as important because they would look at it to decide whether to read the email or not. Sheila begins her email with a formal greeting 'good morning'. Her request is unmitigated and realised in the imperative form (line 5). 'Please' in the initial position intensifies the illocutionary force of her request. She also does not provide any reason for her request. The absence of mitigating strategies when making requests of colleagues of equal status or near equal status could potentially cause a friction in working relationships. However, the use of a formal greeting, as seen in this email, could be interpreted as showing respect to her recipients whom Sheila only knows by the name at the bottom of an email or in the telephone directory. It can also suggest an attempt to construct a positive working relationship by reducing the social distance and at the same time establishing solidarity with all the recipients. In addition, she signs off with her formulaic closing 'regards' and appends her signature in this email, which can also indicate a further attempt to do solidarity.

In the next example (Email 7.25) to a close colleague, Patrick requests Kate to find the information he needs, a task which is considered a routine in Kate's daily job.

Email 7.25 (SP35) NZ2

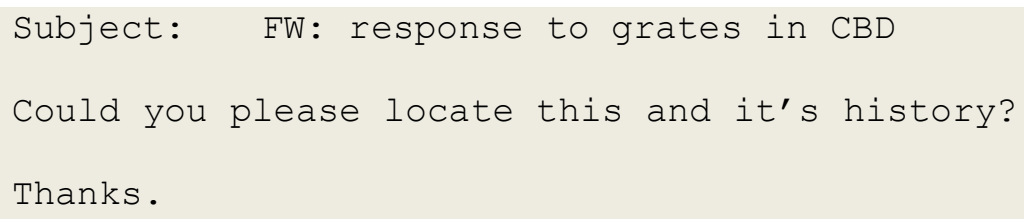


Patrick does not include a greeting in this email to his close colleague, Kate, and launches straight into his request which is realised in the interrogative 'could' form (line 3). The email that ends with 'thanks'could indicate three things: first, it is to thank Kate in advance for her cooperation; second, it is a strategy to balance the disequilibrium of getting someone of equal status or near equal status to do something (Spencer-Oatey, 2008); and third, Patrick attends to Kate's equity rights (i.e. not being unduly imposed upon).

In contrast, Email 7.26 demonstrates that more work is done when requesting a close colleague of equal status or near equal status to do something that is not a routine task.

\section{Email 7.26 (CSO8) NZ2}

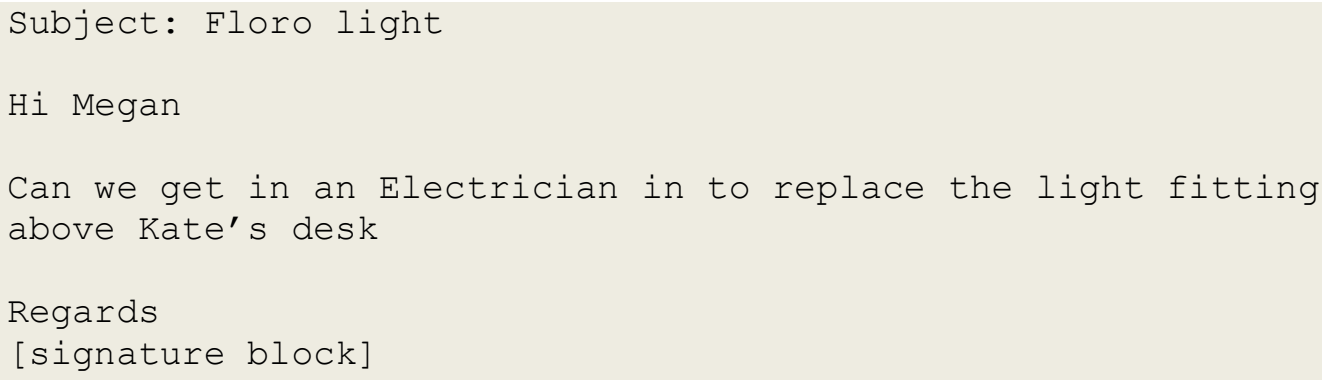

In this email, Sheila requests Megan to replace one of the light fittings in her department, a request which is not considered a routine task. This request is realised in the interrogative 'can you' (line 5). Although the request is direct and there is no evidence of use of any mitigators, two strategies are used to play down the imposition of the request. The inclusion of a casual greeting 'hi' followed by the recipient's name could be interpreted as Sheila's attempt to construct solidarity with her recipient. Sheila uses the pronoun 'we' when making the request although it is obvious that she wants Megan to call the electrician. Megan's role is to take care of all administrative matters and calling an electrician for repair work is her responsibility, but by using the pronoun 'we', Sheila could be offering Megan an option of calling the electrician herself or getting Sheila to do it. That way, Sheila would not be seen as imposing on her (Megan) to do the job. In other words, she attends to Megan's sense of equity rights (the right not to be imposed upon and not unfairly ordered or taken advantage of). 
One strategy used to construct solidarity with distant colleagues is the use of a grounder when making request. In this last example (Email 7.27) sent to a distant colleague, Ken provides a rationale for his request, a marked pattern in Ken's emails.

Email 7.27 (SK95) NZ2

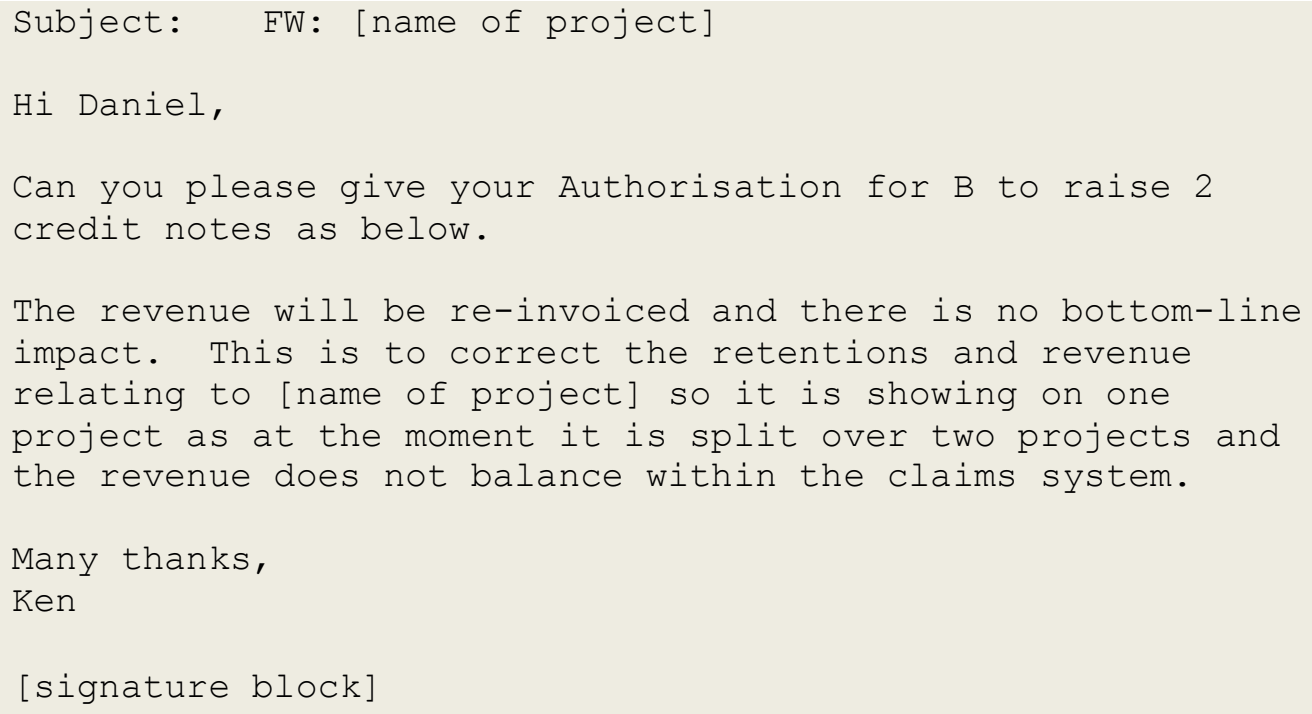

Ken requests Daniel, a distant colleague, to give authorisation to $B$, another colleague, so that $B$ could raise two credit notes to rectify a mistake made in the accounts. His request is realised in the modalised 'can' interrogative form (line 5). In lines 8 to 12, he explains to Daniel why it is necessary for the revenue to be entered into the accounts ('to correct the retentions and revenues') as one project and not as two projects. It is important that all revenues received from clients are correctly accounted for because a wrong entry in the accounting system could have drastic impact on the company's financial accounts. Ken could have omitted the explanation in lines 8 to 12 because he has also forwarded the email from $B$ which gives Daniel a better understanding of what the problem is. However, by giving the brief summary (lines 8 to 12), it could be interpreted that Ken is mindful of Daniel's time and that he attends to his equity rights (i.e. the right of not being unduly imposed upon to do the requested task).

In contrast with the emails discussed above, Email 7.28 from Sheila demonstrates how staff members assert power and authority get work done. 


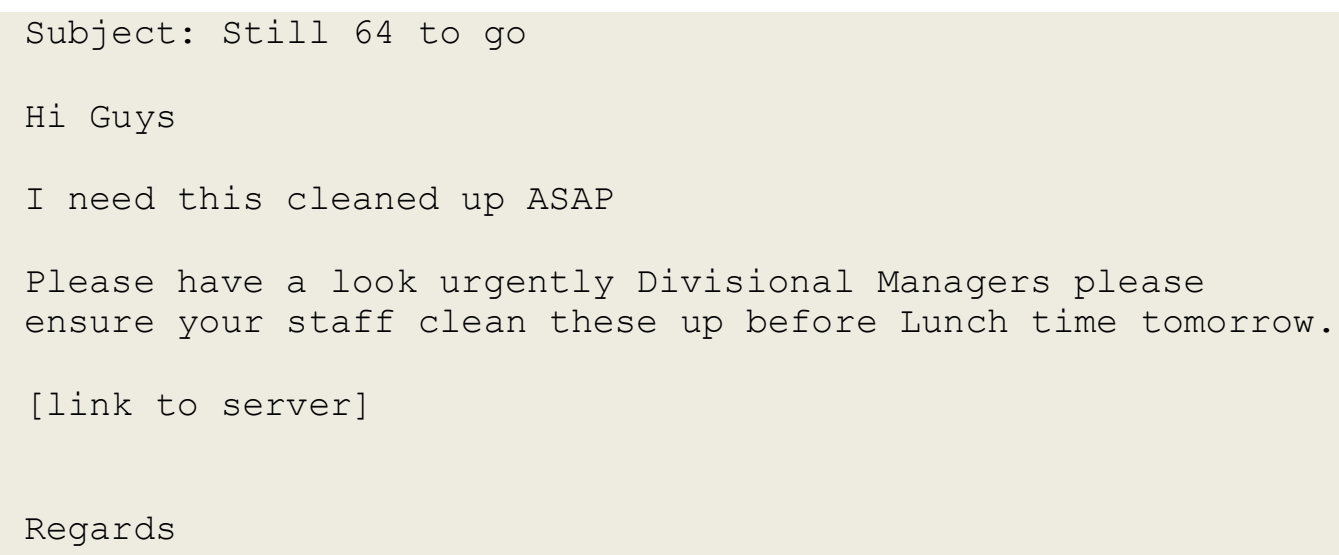

Email 7.28 is sent to other fellow managers. In this email, Sheila chooses the subject heading 'still 64 to go' to clearly signal that a lot of work still needs to be done. As stated earlier (see Email 7.24), staff members reported that the subject heading is an important indicator to decide whether the email needed urgent attention. So this subject heading clearly signals a sense of urgency. In line 5 , she requests her peers to tidy up the summary reports. Her request is realised in the 'need' declarative accompanied with the use of the personal pronoun ' $I$ '. Using these two strategies together, Sheila projects herself as being in charge and as someone with authority. Then in lines 7 to 8 , she tells them specifically what to do: first, she directs the Divisional Managers to check the reports; and two, she instructs them to delete the reports that are irrelevant. The two specific requests are realised in the imperative mood with 'please'. However, the use of 'please' in line 7 appears to further intensify the illocutionary force of her request, rather than a politeness marker. The email is also boosted with four references of time intensifiers 'ASAP' (line 5), 'urgently' (line 7), 'lunch time' (line 8) and 'tomorrow' (line 8). She ends with her formulaic closing 'regards' but omits her name. From the evidence in the email, it clearly shows that getting work done is priority and it appears that Sheila has no intention of managing rapport with her work colleagues.

This section has presented the various strategies (e.g. greeting, mitigating devices) used by peers in Company NZ2 to negotiate requests to peers who are of equal status or near equal status carefully by taking account of the recipient's face. On the other hand, more direct form (e.g. imperative) and boosters are employed to assert power and authority. 
In the next section, I turn my discussion to Company M.

\subsubsection{Company $M$}

As with Company NZ2, staff members in Company $\mathrm{M}$ are also direct when making requests to each other. However, these requests are typically softened by using terms of address such as informal greetings which are common in spoken Malay: 'bro' (a shortened version of brother) or 'kak' (Malay equivalent of sister). Email 7.29 is one example.

Email 7.29 (SS77) M

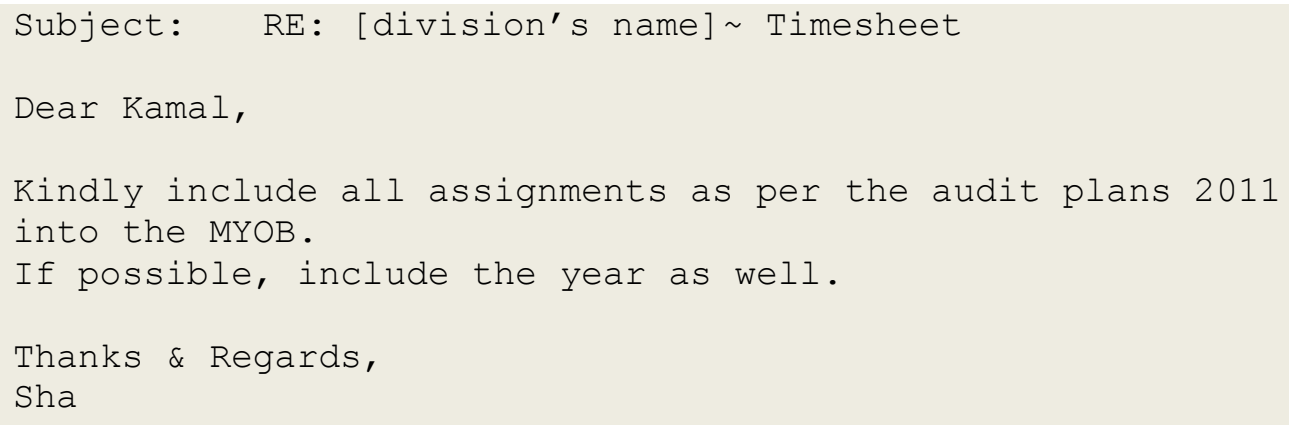

Sharifah begins her email with a salutation (dear) followed by the name of the recipient. The inclusion of a greeting 'dear + first name' suggests that Sharifah attempts to construct solidarity. The use of the salutation 'dear' in Malaysia is considered a sign of doing solidarity. Participants interviewed reported that it is also a form of showing courtesy to their recipients. Sharifah makes two requests: first, she asks Kamal to include all the assignments; and two, she also wants him to key-in the year. Both requests are realised in the imperative form. The first is accompanied by the marker 'kindly' while the second is a mitigated imperative. The request could be tedious and potentially could take a lot of time because Kamal has to manually check the year and insert it into MYOB, an accounting system. Although, she mitigates the second request 'if possible', it is clear that she is 'instructing' Kamal to include the year of each assignment. By hedging her request 'if possible' she could be seen as attending to Kamal's equity rights (Spencer-Oatey, 2000c) as a strategy to balance the disequilibrium of getting a colleague of equal status or near equal status to do something. The use of 'kindly' also appears to soften the illocutionary force of her request (see discussion of the use of 'kindly' in Section 7.3.2.1). Ending the email with the closing 
'thanks and regards' (line 9) could also be interpreted as an additional strategy to avoid causing offence to a peer of equal status.

In this rather short email (Email 7.30), Kamal requests Sharifah, his colleague, to check an audit plan that he has prepared.

Email $7.30(\mathrm{SH} 18) \mathrm{M}$

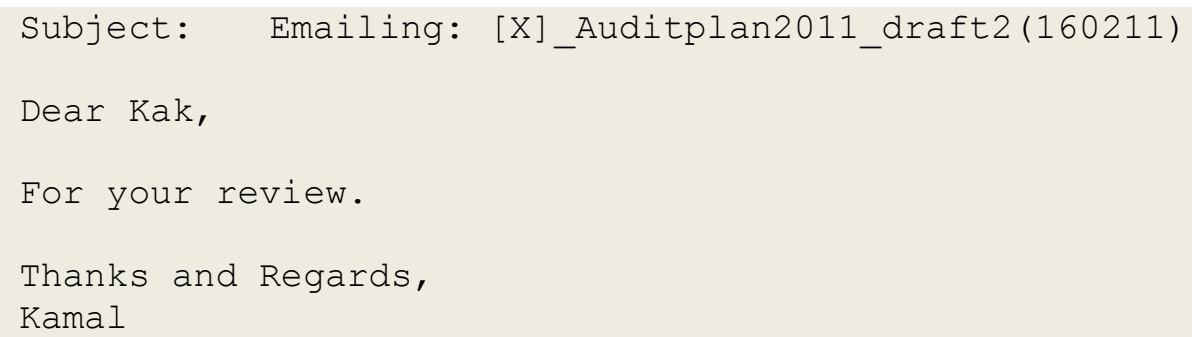

As with the earlier example, salutation is used as a strategy to maintain solidarity and collegiality. Addressing Sharifah with the salutation 'dear' and acknowledging her as 'kak' (Malay equivalent of sister) indicates that Kamal shows both respect and solidarity to his recipient. The term 'kak' is similar to that used in speech and it signals respect, an important practice in the Malay culture. Using the salutation 'dear' and addressing her as 'kak', Kamal can be seen as attending to Sharifah's equity rights (Spencer-Oatey, 2000c) even though his request is realised in the elliptical statement 'for your review'. Seen out of context, this email may be deemed abrupt but according to Kamal in an interview, he had informed his recipient before sending the email. So this email could be read as a polite request. He ends his email with a formulaic 'Thanks and regards' followed by his name.

In another situation, as can be seen in Email 7.31, Geetha appears to assert authority in her reply to Devi, her colleague.

\section{Email 7.31 (CG28f) M}

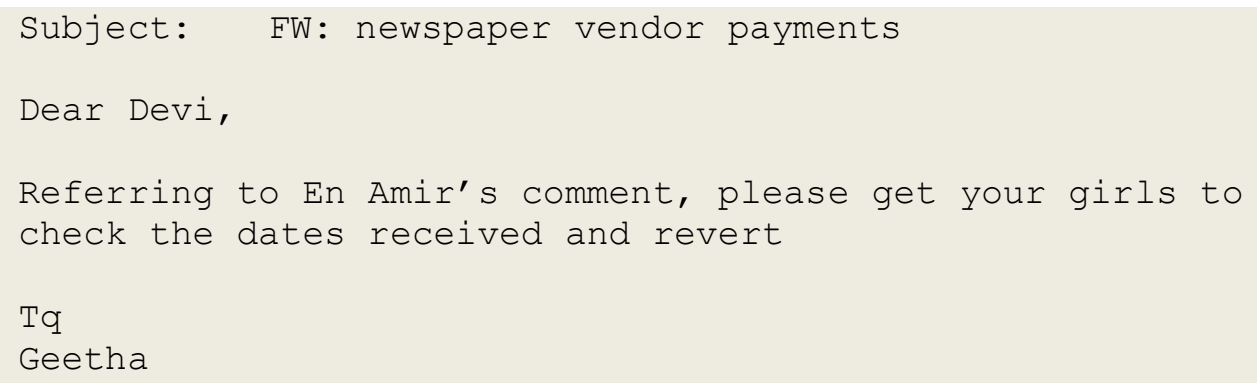


In order to understand what led to this email reply, I give a brief background of this email chain. The accounts department is responsible for issuing payment to the newspaper vendor when an invoice is received from the HR department. On one particular month, payment to the newspaper vendor was overlooked, and this became an issue that involved the two departments. Geetha, the HR Manager, wrote to Devi requesting a summary of payment issued to the newspaper vendor. The email was copied to En Amir, the CEO, who needed to be kept in the loop, as a sign of respect for his position. Before Devi could reply to Geetha, the CEO asked the receptionist, Geetha and his secretary to tell him the procedure for documenting incoming mails. After a few exchanges of emails, Geetha sends Email 7.32 to Devi. She begins her email with a formulaic salutation, a common practice in Company $M$, and uses two strategies to assert authority and to get her colleague to comply with her request: first, she includes the CEO's email in line 5 ('referring to En Amir's comment ...'); and two, she uses the bare mperative form (lines 5 and 6). The first and second verb ('get' and 'check') belong to the material process which construe physical actions while the third verb ('revert') belongs to the verbal process. Using the first verb ('get'), she appears to 'instruct' Devi how to do her job ('get your girls to check') and using the verbal process, she 'tells' Devi to update her. It is clear that Geetha challenges Spencer-Oatey's (2000c) principle of attending to her colleagues' quality face (her competence and ability in accounting), identity face (her role as an Accounts Officer) and sociality rights. Although she signs off 'thank you' ('TQ' in line 8), it does little to repair the damage done by the forceful tone of the email.

In the Malaysian workplace, peers usually employ formal greeting and closing as a sign of constructing solidarity with their colleagues when making requests of each other. On the other hand, peers tend to use a combination of direct forms to assert power and authority when getting their colleagues to comply with the requests.

\subsubsection{Summary of request emails laterally}

Whether the request is considered polite or otherwise by the recipient depends on the social context (Holmes, 1995), including such factors as the relationship with the recipient and the degree of imposition of the request. Hence, making requests of any kind between equals or near equals requires careful attention. 
To demonstrate their sensitivity and tactfulness when making requests of their colleagues of equal or near equal status, the staff members in Company NZ1 use more rapport management strategies when making requests which have high imposition. This supports Holmes and Stubbe's (2003b) findings that more mitigated forms are needed for non-routine and special requests. Staff members also use more linguistic strategies to manage rapport. In the emails discussed above and many others in the data, mitigating strategies, humour, provision of reasons, and an indirect form of requesting are employed when making requests of their colleagues. Hence, the recipient's face sensitivities and sociality rights are attended to.

In contrast, participants in Company NZ2 use more direct forms when making requests of their peers. However, when making requests of distant colleagues, a formal greeting is usually employed. Another strategy employed is the use of a grounder when making requests of distant colleagues. This can also suggest an attempt to construct a positive working relationship by reducing the social distance and at the same time attend to their colleagues' equity rights (Spencer-Oatey, 2000c).

Like Company NZ2, participants in Company M also rely on greetings to attend to their colleagues' equity rights and face sensitivities when making requests to their peers of equal status. However, while participants in Company $M$ tend to use formal greetings ('dear + title + first name'), participants in Company NZ2 usually prefer informal greetings ('hi + first name').

Writers of equal or near equal status engage in a range of strategies to assert authority. The discussion above has provided useful insights into how face-work is accomplished and sociality rights are negotiated in the three workplaces: the participants in Company NZ1 seem to put emphasis on managing rapport while getting work done; conversely, getting work done is given priority in Company NZ2 and Company M. Hence they appear to challenge Spencer-Oatey's (2000c) principle of attending to their peers' face sensitivities and negotiating sociality rights. 


\subsection{Conclusion}

People at work typically aim to achieve both transactional and interpersonal objectives. In this chapter, I have shown how managers and subordinates do power and manage rapport by using various linguistic and pragmatic strategies.

In New Zealand, which has a relatively egalitarian culture, people at work tend to use informal greeting to address one another, taking little account of status or hierarchy. In the two New Zealand workplaces in my study, staff members typically address each other informally, a finding which supports other research conducted in the same country. However, the staff members in the two workplaces manage rapport rather differently. In Company NZ1, the staff members employ more mitigating strategies and indirect form of requests. Rapport is seen as an important consideration in accounting for the way people get things done at this workplace. On the other hand, staff members in Company NZ2 are more direct when making requests of each other, regardless of status and hierarchy.

In contrast, in the Malaysian workplace, politeness and respect for seniority and authority is manifested in the use of terms of address. Respect for authority which is taught from a very young age is carried through to adulthood and becomes a practice even in workplaces. In workplaces where there is a strict observation of hierarchy, subordinates typically show their respect for seniority and authority in a few ways: terms of address, greetings and the use of indirect strategy when making requests. However, in my study, staff members who are subordinate to their recipients typically use direct request form and are more likely to include a greeting and a closing when making requests. Rapport is managed through the use of a formal and deferential greeting.

In sum, this chapter has explored the different linguistic strategies used in email requests in the three workplaces, demonstrating that the different approaches taken may be traced to the workplace culture or CofP. Participants in Company NZ1 tend to employ more rapport management strategies while making requests of each other. They appear to put emphasis on building a harmonious relationship and avoiding conflict. In other words, they prioritise rapport building rather than asserting power in lateral requests. By comparison, participants in Company NZ2 and Company M typically use more direct form when making requests of each other. Although the three workplaces differ in their approaches to maintaining 
interpersonal relationships, the participants in the three workplaces employ the same range of strategies - the use of imperatives, the personal pronoun ' $I$ ' and material processes when asserting power and authority. 


\section{Chapter 8: Conclusion}

Email use has expanded exponentially in the last two decades and it is now a valuable component of the linguistic repertoire of workplace participants. Despite the early claim (i.e. Media Richness Theory) by Daft and Lengel (1984) that email is a lean medium, many researchers have found that email is capable of conveying rich information which contributes to the relational aspect of a message (Abdullah, 2003; Gillaerts, 2012; Ho, 2009; Waldvogel, 2005). For example, Abdullah's (2003, p. 447) study of 250 emails in an organisation in Malaysia testifies that email is "a rich repository of relational information" and not "a cold, impersonal medium". Later, Gillaert (2012, p. 27) who analysed 124 emails from an engineering company in Belgium by drawing on Hyland's (2005) model of Metadiscourse (hereafter MD) finds that the "dominance of interactional MD suggests a strong direct interaction between sender and receiver".

Supporting the claim that email is a rich medium, the aim of this research was to investigate the way in which workplace relations are negotiated, maintained and enhanced in email communication. To answer the research questions, a comparative study was made of three workplaces: two New Zealand workplaces (Company NZ1 and Company NZ2) and one Malaysian workplace (Company M). Company NZ1 was a small event company, Company NZ2 was a multi-national construction company, and Company $M$ was an accounting consultancy firm.

The research findings are based on 282 hours of workplace observations, 67 email survey questionnaires, 40 recorded and transcribed interviews and a dataset of 1745 emails (see summary of the full dataset in Section 3.4.6), and I explain and support the analysis of quantitative data with qualitative data.

In this final chapter of the thesis, I summarise the findings and revisit the research questions. Then I discuss this research's contributions to workplace knowledge. Finally I consider some suggestions for future research. 


\subsection{Summary of findings}

With the aim of studying email communication in three workplaces of two different national cultures, two research questions were addressed in this thesis. The following sections summarise the answers that were revealed by the data analysis.

\subsubsection{Research question one: Relational work in email messages}

The first research question focused on how managers and subordinates accomplish relational work in email messages, i.e. how rapport is established, maintained and negotiated in email exchanges. The data analyses have shown that rapport is managed and maintained through a number of different strategies in order to attend to the recipients' face and sociality rights when making requests in email messages. These strategies included greetings and closings, interrogative mood, use of the pronoun 'we', the presence of mitigating devices and the use of graphical features.

\subsubsection{Greetings and closings}

People usually use greeting and closing in email to establish rapport with each other. Greetings and closings are used in all three workplaces in varying proportions and for different purposes. The different types of greeting used in the three workplaces indicate and construct the different attitudes towards interpersonal relationships at work. In the two New Zealand workplaces, the informal greeting ' $\mathrm{Hi}$ ' + first name generally indicates and constructs the egalitarian New Zealand attitude to interpersonal relationships at work. The widespread use of the informal and friendly ' $\mathrm{Hi}$ ' in Company NZ1 and Company NZ2, regardless of status and seniority, further signals solidarity and collegiality at the workplaces. For example, as demonstrated in Email 6.18, Ken greets his General Manager with 'Hi' and addresses him with his first name. However, none of the participants in Malaysia greeted their superiors with an informal greeting or with the first name.

Even in the same country (New Zealand) which practises an egalitarian culture, the choice of greetings and closings in the two New Zealand workplaces show a contrast. It was shown that staff members at Company NZ2 used more formal greeting (i.e. Good morning or good afternoon) rather than more informal ' $\mathrm{Hi}$ ', for example, when writing to a group of work 
colleagues. In addition to a more formal greeting, the formal closing 'Regards' rather than the informal 'Cheers' as a closing expression was preferred. Also in Company NZ2, signing off using the initial of first name was not a common practice. Emails in Company NZ1 suggest a more relaxed and collegial atmosphere where the informal greeting ' $\mathrm{Hi}$ ' and closing 'Cheers' were used and signing off with the initial of the first name was found in eight percent of the emails. This informal style could further signal and construct the collegial atmosphere and close interpersonal relationships of the members in this CofP.

In contrast, in Malaysia, the use of formal greetings such as 'Dear' + title + first name and 'Dear' + honorarific epithet signal and construct deference and respect towards the recipients. This finding is consistent with Lee's (2011) observation on key concepts and norms of politeness among the Chinese community of Singapore which shows that respect for seniority and authority is considered very important in workplaces. As discussed in Chapter 6, the use of 'dear' is viewed as negative rather than positive politeness by New Zealand people (Waldvogel, 2007), whereas an absence of the salutation 'dear' in the Malaysian context could be interpreted as being impolite and disrespectful. Also in Company $M$, it was shown that greeting and closing were more likely used by subordinates when writing to superiors and colleagues who were more senior. As noted by Pan (1995, p. 479) who observes Chinese official settings "the [institutionalised] power of one's rank is the dominant factor that determines politeness behaviour patterns". This observation is confirmed by Abdullah's (2003) research and the data in my study.

Based on the above comparison, I argue that the use of greeting and closing is seen as an important means of establishing rapport with the recipients. However, the workplaces in the two different cultures use them differently. As discussed, the use of a formal greeting ('Dear' + title + first name or 'Dear' + honorific epithet) and closing ('Regards and thanks' + name) in the Malaysian workplace suggests that hierarchical relationships are given greater importance by the staff members while also constructing deference and respect towards the recipients. On the other hand, the egalitarian New Zealand culture seems to promote the use of an informal greeting ('Hi' + first name) and closing ('Cheers' + name) which constructs a more collegial atmosphere and close interpersonal relationship. 


\subsubsection{Interrogative mood}

The way in which requests are worded is another way of signalling how rapport is managed and enhanced. Writers employ many strategies to make their messages less facethreatening and attend to the recipients' face sensitivities and association rights. One of the strategies used is phrasing requests in the interrogative mood. As demonstrated in Section 7.3.2.2, it was shown that more than half of the requests were realised in the interrogative mood in the two New Zealand workplaces. Of the requests which were realised in the interrogative mood, the most common form is the interrogative with 'can'. According to Blum Kulka (1987, p. 136), the "highest level of politeness" is achieved by the use of the interrogative mood 'can you'. This indirect form appears to soften the force of the request and staff members could be seen as attending to the recipients' face - identity face and quality face - and sociality rights - association rights and equity rights (Spencer-Oatey, 2000c). In contrast, the interrogative mood was the least preferred request form in Company $\mathrm{M}$, with only 15 percent of the requests realised in the interrogative form.

Phrasing requests in an interrogative mood was the choice preferred in the two New Zealand workplaces. On the other hand, the preferred form for participants in Malaysia was the imperative mood (see Section 8.1.2.1). Thus, practices around the use of interrogative mood differ between New Zealand and Malaysia, and this has implications for the workplace culture.

\subsubsection{Use of inclusive 'we'}

When making requests, superiors and subordinates in the New Zealand workplaces constantly attended to the recipients' face and sociality rights by reducing the degree of face-threat of messages. As discussed in Chapter 7, in Email 7.2, Andy (Company NZ1) begins the request with the pronoun 'we' (Also, we'll need to re-do this page ...), emphasising that both she and Teresa (the recipient) would be doing the task together as a team. When Andy uses the inclusive pronoun 'we', it functions to align herself with Teresa, which also expresses solidarity. Similarly, in Email 7.18, Lesley uses the pronoun 'we' ('Shall we try to get it out for the start of [month]?') when she requests that her colleagues provide her an article for the company's newsletter. By doing so, she attends to the equity rights and association rights of her colleagues that they would be doing the requested task together as 
a group. The use of inclusive 'we' is also regarded as an indicator of cooperativeness between colleagues (Planken, 2005) and team work (File, 2013).

However, in the Malaysian workplace, the inclusive pronoun 'we' seldom occurred. In the example 'When are we wrapping this up?' (Email 7.10), the use of exclusive pronoun 'we' indicates that the CEO expects the subordinate and his HOD to complete the report for the company's client. Similarly, in another example the use of the pronoun 'you' as in 'Can both of you ...' (Email 7.11) indicates that the CEO expects two of his subordinates to work together on an article for the company's internal newsletter. Using the pronoun 'you' (i.e. 'can you') rather than the inclusive 'we' (i.e. 'can we') seems to suggest that the CEO focuses on power and authority.

The use of inclusive pronoun 'we' in the New Zealand data shows that superiors and subordinates reduce the degree of face threat of messages while the use of exclusive pronoun 'we' and second person pronoun 'you' in Malaysia suggests that emphasis is placed on getting work done. The use of the latter two pronouns in the Malaysian data also seems to violate Spencer-Oatey's (2000c) principles of attending to the recipients' face sensitivities and sociality rights.

\subsubsection{Mitigating devices}

One way of softening the effect of requests is the use of mitigating strategies which includes hedging devices such as modal verbs ('might', 'perhaps') and lexical items ('possible', 'probably', 'maybe') to project tentativeness. Other softening devices include the use of interpersonal metafunction (Halliday \& Matthiessen, 2004) such as mental process (through the use of verbs such as think, notice) and the provision of justification. Emails written by the members in Company NZ1 and Company NZ2 show that a number of mitigating devices were used to soften the force of the requests made. The analyses also show that mitigating devices, the use of mental processes and the inclusion of justification were present regardless of the hierarchical status of the writer which suggests that solidarity and collegiality seem to be given more emphasis than status in the egalitarian New Zealand context. All these mitigating devices function to maintain rapport because their absence could challenge the recipient's sense of equity rights (Spencer-Oatey, 2000c, p. 18). 
Meanwhile, the Malaysian participants used very minimal mitigating strategies when making requests of their work colleagues. Consistent with Abdullah's (2003) findings, most requests in my Malaysian data were unmitigated and realised in limited forms, usually in the imperative. However, the marker 'kindly' did occur and seemed to play down the impact of the requests made (House \& Kasper, 1980). As discussed in Section 7.3.2.1, the word 'kindly' could be attributed to the translation of the Malay equivalent, 'harap'.

In sum, the New Zealand participants used various mitigating strategies to attend to the recipients' face sensitivities and sociality rights while the Malaysian participants relied on the marker 'kindly' to reduce the degree of face threat of a request.

\subsubsection{Humour}

Previous research has shown that humour helps to create team spirit, builds rapport and reaffirms group solidarity (Holmes, 1999, 2006; Holmes \& Marra, 2002; Holmes \& Stubbe, 2003b; Ladegaard, 2009; Schnurr, 2005). As discussed in Chapter 4, humour was a distinctive practice of the CofP in Company NZ1, but it was not a common practice in Company NZ2 and Company M. In Company NZ1, humour was used by staff members to gain "positive cooperative intent" and attend to work colleagues' face sensitivities and sociality rights (Holmes \& Stubbe, 2003b, p. 115). When making requests upward, humour was used as a strategy to attend to the superior's face sensitivities and equity rights (the right of not being subjected to undue imposition) (Spencer-Oatey, 2000c) as demonstrated in Email 7.13.

\subsubsection{Self-denigration}

Like humour, self-denigration also contributes to team collegiality (Holmes \& Stubbe, 2003b; Schnurr \& Chan, 2011). In my study, this strategy is only found in Company NZ1 data. As discussed in Section 7.6.1, it is not a common practice for a colleague in an organisation to admit his/ her own incompetence. However, Lesley used this strategy with care which could be interpreted as her sensitiveness towards paying attention to interpersonal and relational work (see email 7.19). In another situation, Andy, the CEO, used this strategy as an excuse 
for a memory lapse, 'Alzheimers moment', when asking Susan to remind her of an important sponsor for a company's event.

\subsubsection{Graphical features}

Prosodic features such as pitch, stress and volume which are present in spoken language are absent in emails. Due to their absence in emails, graphical features such as capital letters, emoticons (smileys), non-standard punctuation and use of colour are used to convey paralinguistic cues. These graphical features are considered distinctive features in email (Abdullah, 2003; Crystal, 2006). The use of emoticons was present in the data from Company NZ1 but not in the other New Zealand workplace. The use of emoticon did not occur in formal work related emails in Company M. Only emails announcing social events for the company sent by the Sports Club in Company $\mathrm{M}$ were informal and had a lighter note as demonstrated in Section 4.5.3. While all typical emails related to work were written in the conventional style, emails sent by the Sports Club contained some graphical features (e.g. use of colourful fonts, different font types and sizes, pictures) and these could be interpreted as a strategy of building rapport with work colleagues. Staff members interviewed said that they feel happy receiving emails with smiley emoticons which in their opinion diffused the formality of the email. Even though Angell and Heslop (1994, p. 111) state that smileys "are the equivalent of e-mail slang and should not be used in formal business e-mail messages", the use of emoticon in the email messages present in the data collected from Company NZ1 could be interpreted as further signalling and constructing collegiality and solidarity in this CofP. As discussed in the comparison above, the presence of graphical features in emails could suggest a strategy of rapport building.

\subsubsection{Research question two: Construction of power in email messages}

The second research question concerned how managers and subordinates construct and instantiate power and negotiate status-based workplace relationships in email communication. The data analyses have shown that managers and subordinates construct power and authority using various strategies: imperative mood, use of personal pronoun ' $I$ ' and boosting devices. 


\subsubsection{Imperative mood}

One way managers construct power and authority is through the use of the imperative mood (Ladegaard, 2011). As demonstrated in Chapter 7, the use of the imperative mood and the absence of mitigating or softening devices could be interpreted as managers placing more emphasis on getting the job done which suggests that they may not be interested in building rapport with their subordinates. The presence of boosting strategies further confirms this observation. In other words, managers have a tendency to violate SpencerOatey's (2000c) principle of attending to their subordinates' face sensitivities and sociality rights. For example, in Email 7.3 to all the HOEs, Andy, the CEO in Company NZ1, uses the imperative mood to stress the importance of writing short and concise reports. In that one email she uses six bald-on record imperatives to make her message clear. In another example (Email 7.9) extracted from Company M, the CEO, Amir, reminds his subordinate the importance of giving facts and figures when writing reports to their clients. The use of the imperative mood clearly shows that Amir places more importance on getting the job done correctly than maintaining rapport with his subordinate.

Also discussed in Section 7.3.3, the use of 'please' with an imperative mood could be regarded as intensifying the illocutionary force of a request (Economidou-Kogetsidis, 2011; Sato, 2008; Waldvogel, 2005). In the first example (see Email 7.7) taken from Company M, Amir, the CEO, requests his subordinates to make sure that all final reports and audit reports are saved on the server. The use of 'please' in 'please ensure ...' seems to intensify the illocutionary force of the request encouraging all his subordinates to comply with his request. In the second example also extracted from Company $M$, Sharifah requests her colleague to replace the wrong version of an audit plan with the amended version. Using the imperative mood and intensifying the illocutionary force of the request with the marker 'please' ('Please replace the earlier version to this one - amended after the Department Meeting'), she constructs herself as someone with authority. In the third example taken from Company NZ2, Sheila, the Senior Administrator, reminds her colleagues who are Divisional Managers and senior managers to show the company's monthly video to their subordinates during the bimonthly Toolbox meeting. She uses the imperative mood and the marker 'please' ('please unsure [ensure]') which seems to boost the illocutionary force of the request. 
The findings show that the managers and subordinates do power by employing the use of bald-on record imperatives and the marker 'please'. From my analysis, the marker 'please' tends not to have a politeness effect when it is used in an asymmetrical relationship.

\subsubsection{Use of personal pronoun ' 1 '}

Power and authority can also be expressed when managers and peers use the personal pronoun ' $l$ ' coupled with a particular material process (Halliday \& Matthiessen, 2004). According to Alsree (1997, p. 180), "'I' is used as the exclusive source of reference" and is considered as a feature in the power of language. Similarly, Holmes, Marra and Vine (2011) demonstrates that the use of ' $I$ ' in narratives is one way in which Pakeha male leaders construct themselves as providing good leadership. In Email 7.4, Andy, the CEO of Company NZ1, sends an email to all staff members to inform them of a solution to a car park problem which once caused an email war between a few staff members. In this email, she uses the personal pronoun ' $\mathrm{I}$ ' and material processes such as 'I have reviewed ...' and 'I polled ...' to instruct the staff members to abide by her decision. By conveying her request through material processes rather than other processes such as 'I think' or 'I feel', she makes her actions explicit.

In another example discussed in Email 6.21, Chandran, the HOD in Company M, constructs himself as an authority figure when he uses the personal pronoun ' $I$ ' to reprimand his subordinate for not complying with the given guidelines when preparing a report. In this email, he also uses bare imperatives to make his message explicit.

Sheila, from Company NZ2, also asserts power and authority in Email 7.28 to other fellow managers when she requests her peers of equal or near equal status to complete the summary reports and to delete reports which are not relevant. Her request is realised in the 'need' declarative accompanied with the use of the personal pronoun ' 1 '.

In sum, the personal pronoun 'I' may assert an authoritative position so that using it, managers and peers construct themselves as authoritative figures and assert power. 


\subsubsection{Boosting devices}

There are various boosting devices that managers and subordinates can use to assert power and authority in email messages, including the use of modals of obligation, the presence of time intensifiers, messages that are given high priority and messages that are written in capital letters.

Modals of obligation such as 'should' and 'must' are frequently used by staff members to enact authority and power and these are expressed explicitly to specify what others should do or should not do. For example, in an email to all her HOEs, Andy, the CEO in Company NZ1, stresses the importance of good customer service 'we should always ...' to their clients and sponsor. In another email, Andy directly points out a mistake that her manager made in a contract 'you should never do this ...'

In addition to using obligatory modality, other boosting devices such as time intensifiers, e.g. 'ASAP' and 'immediately', further indicate the construction of status in the workplace. In Email 7.28, Sheila uses three references of time intensifiers 'ASAP', 'lunch time' and 'tomorrow' to boost the illocutionary force of her email requesting her colleagues who are managers to complete all reports.

While the use of graphical features such as emoticons and colourful fonts are used to build rapport with work colleagues (see Section 8.1.1.7 above), they can also be used as boosting devices. Capital letters in emails can be used to convey emphasis or they can be interpreted as 'shouting' if a message is all capital letters (Alsree, 1997). For example, Amir, Company M's CEO, said that he uses red font and capital letters in his email "if I am angry". In another example, Megan, who is not a manager in Company $M$, writes an email to all the staff members in capital letters. The entire email which is written in capital letters clearly suggests that she asserts authority to ensure that all Daily Job Records (DJR) are submitted punctually to avoid delay in payment. With the presence of additional boosting devices: modal ('MUST') and time intensifier ('9 AM THIS MORNING'), she shows that she challenges Spencer-Oatey's (2000c) principle of attending to her work colleagues' face sensitivities and sociality rights, giving precedence to getting the job done.

Thus, we have seen how both managers and subordinates do power and authority through the use of boosting devices as discussed above. 
After reviewing the two research questions, I now consider the various areas that my research can contribute to.

\subsection{Contribution of research}

The current research drew on existing literature on email communication and politeness theory. The research contribution is discussed in relation to three areas: $\mathrm{CMC}$, interpersonal communication and workplace culture.

\subsubsection{Contribution to research on computer-mediated communication (CMC)}

According to Media Richness Theory, email communication is a lean medium used primarily for conveying transactional information and is incapable of communicating rich information and fostering social relationships (Daft \& Lengel, 1984). However, the analyses in this thesis show that email communication serves a wide range of purposes: giving information, making requests; sending social invitations; constructing collaborative humour; teasing; expressing thanks; congratulating and apologising. The data in Chapter 5 shows the different proportions of usage in email communication to achieve various functions across the CofPs. The findings in this chapter supports Waldvogel's (2005) claim that email plays an important role in organisational communication.

In addition, in Chapter 4, the data analysis demonstrates how email was used for both transactional and interpersonal purposes. When emails were used to do interpersonal work, they constructed solidarity and built rapport amongst the members of the CofPs. For instance, morning tea shouts and other social events were announced via emails, as demonstrated in Company NZ2 and Company M.

Besides performing transactional work, email communication can also carry relational information and this is achieved through the use of greetings and closings in emails and the use of various linguistic features such as mitigating devices, grounders and different mood choices. Occurrences of humour were also evident in the emails as a vehicle for constructing solidary relationships. These were discussed in Chapters 4 and 7 . The analysis of greetings and closings in Chapter 6 further contributed to the body of research that questions the notion that email is a lean medium and supports other researchers' findings that workplace 
email can do both transactional and affective work (Abdullah, 2003; Fletcher, 2011; Ho, 2009; Jensen, 2009; Kankaanranta, 2005; Waldvogel, 2005). It shows that the presence of appropriate forms of greeting can contribute to positive relationship building. In short, this study has clearly demonstrated that email is a rich medium which is capable of fostering social relationships.

There has been very little research on CMC in Malaysia apart from a couple of studies on email communication (Abdullah, 2003; Alsree, 1997). My study of written communicative behaviour of New Zealand and Malaysian people extends our understanding of email communication at the Malaysian workplace which is also a contribution to CMC.

\subsubsection{Contribution to research on interpersonal communication}

This thesis employed rapport management as an analytic framework. In Chapter 2, I presented an overview of politeness theory and explained the position I took for the study. I selected rapport theory as an analytical framework for two reasons: 1) to account for the cross-cultural dimension found in the present study; and 2) to provide a richer interpretation when the analysis is expanded from an utterance level to a discourse level, especially in CMC contexts. In addition, I also incorporated a CofP approach as the contextual focus to give a better understanding of the three workplaces. The assumptions in analysing authentic emails for interpersonal dimension can be summarised as follows: first, rapport is considered as a negotiated process in an on-going interaction, including email communication; second, a CofP can develop in each workplace if the three dimensions of a CofP (regular interaction, shared goal and a set of shared linguistic and non-linguistic practices) are met; and third, an analysis within rapport theory and CofP approach allows me to focus on a micro (discourse level) and macro (context and culture level) analysis to present similarities and differences between the workplaces.

As discussed in the chapters which considered the use of greetings and closings (Chapter 6), and lexico-grammar and linguistic strategies (Chapter 7), rapport was constructed by the writers with their recipients in the email messages. 
From the analyses, I have demonstrated that both rapport theory and CofP approach provide useful analytical frameworks in exploring rapport management (maintenance and enhancement) in workplace discourse. Rapport theory is a robust framework as it allows an analysis beyond illocutionary domain and is applicable from a cross-cultural perspective. Applying the CofP framework enabled me to analyse each workplace individually and how the staff members constructed themselves as core members in the CofPs. All three workplaces could be classified as CofPs because each workplace fulfilled the three crucial criteria of a CofP as demonstrated in Chapter 4. As I noted in Chapters 4, 6 and 7, interpersonal communication and workplace culture are inter-connected.

\subsubsection{Contribution to workplace culture research}

The findings discussed in this study are useful for understanding people's linguistic and nonlinguistic behaviours from three different workplaces or CofP.

Workplace culture as demonstrated in Chapter 4 influences how people manage rapport and do power. For example, for the CofPs in Malaysia, inequality in power is accepted as normal, therefore, people do not question superiors' authority, rights and entitlement to privileges. As discussed in Chapter 7, it appears that Amir took advantage of his hierarchical position and legitimate power as the CEO and his legitimate power to make a 'bald-on record' requests. In contrast, the participants in New Zealand employed various linguistic strategies to make requests as there was more evidence that the CofPs in Company NZ1 were doing more relational work (i.e. the use of humour, mitigation and provision of justification) when making requests of work colleagues. By employing these additional strategies they attended to the recipients' face and sociality rights. It is also interesting to note that the CEO in Company NZ1 also employed various linguistic strategies when making requests of her subordinates. Ho (2009, p. 38) who studied emails in a public academic institution in Hong Kong refers to the presence of leaders' rapport management work as "open to a polite interpretation" (Locher \& Watts, 2005, p. 26) and help leaders "construct the identity of a polite leader".

The analysis of the use of greetings and closings in Chapter 6 has shown the importance of developing an understanding of how people greet each other in different workplace cultures. 
Existing literature on greetings and closings in emails suggests that the use of first names and informal forms of greetings and closings such as 'Hi' are considered a norm in the egalitarian nature of New Zealand society (Waldvogel, 2005). The use of the salutation 'dear' and title signal negative politeness rather than positive politeness. In contrast, in Malaysia, a high context culture (see Section 6.2.1) that emphasises the use of titles and forms of address, the use of first name alone is deemed impolite and it is rude to address someone who is senior (age) or in a superior position by the first name. For example, Alsree (1997) cites examples of Malaysian managers who worked in a multinational company were unwilling to address their superiors by their first names. In my data, the use of informal greetings and closings by the CofPs in the two New Zealand workplaces are seen as constructing a solidary relationship with the interactants. The use of formal and deferential greetings and closings by the CofP in the Malaysian workplace is a sign of showing respect and deference.

In addition, my present study of three CofPs presented a rich source of data on how the New Zealanders and Malaysians communicate within their work cultures. The data and information in my study will help people understand communication, specifically email communication, and how it works in their own contexts. For example, learning how to make requests in emails in different workplace cultures or CofP will put a newcomer on a better footing. Previous researchers have shown that non-native speakers of English lack proficiency in using appropriate pragmatic forms of the English language when making requests through emails (Abdullah, 2003; Biesenbach-Lucas, 2007; Economidou-Kogetsidis, 2011; Hendriks, 2010). In Chapter 7, the data analysis showed that writers in the New Zealand workplaces in my research used different degrees of directness (the use of interrogatives rather than imperatives), mitigating devices and grounders to make requests. For example, when making requests, the New Zealand writers in my study tended to use the modalised interrogative forms 'could you ...?' or 'can you ...?' rather than the imperative form. Another observation is that New Zealand writers also preferred to provide justification (grounder) for the request, especially if the request was made upward, i.e. from a subordinate to a superior. In the Malaysian data, writers preferred the imperative form 'please find attached ... for your review'. They also did not provide justification for the requests made. From a pragmatic point of view, this seems to contribute to a "brusque e- 
mail effect" and may even be deemed as impolite (Economidou-Kogetsidis, 2011, p. 3209). Increasing awareness of the complexities of lexical modifiers and syntactic modification can help non-native speakers of English to be more informed and make appropriate choices when they construct request emails. Having this knowledge gives non-native speakers an added advantage because they have a better footing when liaising with native speakers of English. In addition, these findings can facilitate in drawing up suitable materials for language training.

The cultural differences - workplace culture and national culture - found in this thesis relate to a relatively restricted domain and the findings were based on an analysis of just three case studies. Nevertheless, they are important in providing insights especially for people from Malaysia and New Zealand. I hope that these findings can contribute to a better understanding of inter-cultural and workplace communication, especially in email communication. These findings can also help reduce misunderstandings when people move from one workplace to another. Understanding a new workplace culture and engaging in "ways of doing things, ways of talking, beliefs and values" can help move a newcomer forward from being a peripheral participant to a full participant (Eckert \& McConnell-Ginet, 1999, p. 186).

\subsection{Future research}

This study is essentially case studies of three workplaces in two countries. Because the data analysis in the present study is extracted from just three workplaces in two countries, one obvious way is to do a similar sort of study in different contexts, more workplaces in New Zealand and Malaysia. The findings and conclusions presented in this research should be regarded as suggestions only because it is exploratory in nature. However, given the rich data collected, the findings can be used as a springboard for similar future studies.

Further research based in different contexts and in different types of workplaces is warranted to see to what extent the findings in this present research are applicable to other workplaces or CofPs.

Taking these limitations into consideration, I suggest a number of possibilities for future research. One possible extension of the present study would be to undertake similar 
research in other companies in New Zealand and/ or Malaysia or other CofPs to verify the findings. It may also be interesting to compare results from replications of this study in multi-national companies which have branches in both countries. As this study focused on only two national cultures, it would be useful to include more countries in future research to cross-validate the results. Similarly, a study of different CofPs would also be useful for comparison.

Another possible extension would be to conduct further research in different contexts and in different types of workplaces since this study addresses only three types. As noted in Chapter 2, many studies on workplace discourse, especially email communication, are conducted in English speaking countries,therefore, more research in non-English speaking countries, such as in the Asian region, is necessary to provide a wider inter-cultural perspective. This would also facilitate better inter-cultural understanding and it would also have the potential to lay the foundation for investigating breakdown in email communication or miscommunication that arises from cultural differences.

The present study focused on the various strategies employed in email communication by thirteen staff members in three CofPs in two very different countries to construct solidarity and do power. An area of research that warrants further investigation is a focus on gender differences on email communication in two different cultures and/ or CofPs. According to some researchers, women use more strategies to compensate for the lack of socioemotional cues than men (Brosnan, 2006; Gillaerts, 2012; Holmes \& Stubbe, 2003a; Morpeth, 2012; Panteli \& Seeley, 2006; Waldvogel, 2007), so it would be interesting to investigate if this finding holds true from an inter-cultural perspective in order to draw conclusions with regard to the role of gender on workplace emails. This knowledge could also provide a better understanding of how different genders write from an inter-cultural point of view.

\subsection{Concluding remarks}

In this thesis, I have demonstrated how each CofP in the two countries manages rapport and does power through a range of linguistic and pragmatic strategies. By employing the CofP framework, this thesis has shown how members develop their own identity and shared 
practices and how these practices are constantly renegotiated in order to align with the enterprise of their CofP. With the advancement of technology, email communication has become an established and significant component in workers' communicative repertoire and the findings in the present research have contributed to a better understanding of how people achieve interactional goals.

My study has also shown that the consideration of rapport management deserves more attention in workplace settings, especially with regards to how rapport is realised in various modes of communication across cultures. The effect of cultural diversity on the practices of rapport management requires further investigation because different cultures establish rapport and solidarity differently. 


\section{References:}

Abdullah, A. (1996). Going global: Cultural dimensions in Malaysian management. Kuala Lumpur: Malaysian Institute of Management.

Abdullah, N. A. (2003). Relational communication in organisational email: A Malaysian case study. Unpublished PhD thesis. University Malaya, Kuala Lumpur, Malaysia.

Abdullah, N. A. (2006). Constructing business email messages: A model of writers' choice. ESP Malaysia, 12, 53-63.

Agar, M. H. (1996). The professional stranger: An informal introduction to ethnography (2nd ed.). San Diego: Academic Press.

Alsree, Z. S. A. (1997). Language and power: A critical analysis of email text in professional communication. Unpublished PhD thesis. University of Reading, Reading, UK. Retrieved from http://helicon.vuw.ac.nz/login?url=http://zebedee.vuw.ac.nz/ebooks/Alsree Langua gePowerEmail.pdf

Angell, D., \& Heslop, B. (1994). The elements of e-mail style. Reading, MA: Addison-Wesley.

Angrosino, M. (2007). Doing ethnography and observational research. London: Sage.

Arundale, R. B. (2006). Face as relational and interactional: A communication framework for research on face, facework and politeness. Journal of Politeness Research, 2(2), 193216. doi: 10.1515/PR.2006.011; (AN 21971190)

Ashkanasy, N. M., Trevor-Roberts, E., \& Kennedy, J. C. (2004). The egalitarian leader: Leadership in Australia and New Zealand. In D. Tjosvold \& K. Leung (Eds.), Leading in high growth Asia (pp. 231-252). Singapore: World Scientific.

Asmah, H. O. (1982). Language and society in Malaysia. Kuala Lumpur: Dewan Bahasa dan Pustaka.

Austin, J. (1962). How to do things with words. Oxford: Oxford University Press.

Bardsley, D. (2012, 29 February). New Zealand politics and life infused with tea terms, The Dominion Post. Retrieved from http://www.victoria.ac.nz/lals/resources/publications/29Feb2012.pdf 
Bardsley, D. (2013). Book of New Zealand words. Wellington, New Zealand: Te Papa.

Bargiela-Chiappini, F., \& Harris, S. H. (1997). Managing language: The discourse of corporate meetings. Amsterdam: John Benjamins.

Baron, N. (1998). Letters by phone or speech by other means: The linguistics of email. Language and Communication: An Interdisciplinary Journal, 18(2), 133-170. doi: 10.1016/S0271-5309(98)00005-6 <http://dx.doi.org/10.1016/S0271-5309(98)00005-6>

Baym, N. (1995). The emergence of community in computer-mediated communication. In S. G. Jones (Ed.), CyberSociety: Computer-mediated community and communication (pp. 138-163). Thousand Oaks, CA: Sage.

Baym, N. (1998). The emergence of on-line community. In S. G. Jones (Ed.), CyberSociety 2.0: Revisiting computer-mediated communication and community (pp. 35-68). London, UK: Sage Publications Inc.

Bell, A. (2000). Maori and Pakeha English: A case study. In A. Bell \& K. Kuiper (Eds.), New Zealand English (pp. 221 - 248). Wellington: Victoria University Press.

Bennett, J. A. W. (1943). English as it is spoken in New Zealand. American Speech, 18(2), 8195. doi: $10.2307 / 486595$

Biesenbach-Lucas, S. (2007). Students writing emails to faculty: An examination of epoliteness among native and non-native speakers of English. Language Learning \& Technology, 11(2), 59-81.

Bloomer, A. (2010). Designing a questionnaire. In S. Hunston \& D. Oakey (Eds.), Introducing applied linguistics (Concepts and skills) (pp. 145-152). Oxon, New York: Routledge.

Blum-Kulka, S. (1982). Learning to say what you mean in a second language: A study of the speech act performance of Hebrew second language learners. Applied Linguistics, 3(1), 29 - 59.

Blum-Kulka, S. (1987). Indirectness and politeness in requests: Same or different? Journal of Pragmatics, 11(2), 131-146. doi: 10.1016/0378-2166(87)90192-5

Blum-Kulka, S., House, J., \& Kasper, G. (1989a). Cross-cultural pragmatics: Requests and apologies. Norwood, NJ: Ablex. 
Blum-Kulka, S., Kasper, G., \& House, J. (1989b). The CCSARP coding manual. In S. Blum-Kulka, G. Kasper \& J. House (Eds.), Cross-cultural Pragmatics: Requests and Apologies (pp. 273 - 294). Norwood, NJ: Ablex.

Blum-Kulka, S., \& Olshtain, E. (1989). Requests and apologies: A cross-cultural study of speech act realization patterns (CCARP). Applied Linguistics, 5(3), 196 - 212.

Bönisch-Brednich, B. (2008). Watching the Kiwis: New Zealander's rules of social interaction an introduction. Journal of New Zealand Studies, 6/7 (Special Issue: Watching the Kiwis), 3-15.

Bourdieu, P. (1990). The logic of practice. Stanford: Stanford University Press.

Brannan, M. J. (2007). Sexuality, gender and legitimate peripheral participation: An ethnographic study of a call centre. In J. Hughes, N. Jewson \& L. Unwin (Eds.), Communities of practice: Critical perspectives. London \& New York: Routledge.

Brosnan, M. J. (2006). Gender and diffusion of email: An organisational perspective. In M. Barrett, and Davidson, Marilyn J. (Ed.), Gender and communication at work (pp. 260269). Hampshire: Ashgate Publishing Limited.

Brown, P., \& Levinson, S. (1987). Politeness: Some universals in language usage. Cambridge: Cambridge University Press.

Burns, A. (2010). Action research. In B. Paltridge \& A. Phakiti (Eds.), Continuum companion to research methods in applied linguistics (pp. 80-97). London: Continuum.

Chan, A. C. K. (2005). Openings and closings in business meetings in different cultures. Unpublished PhD thesis. Victoria University of Wellington, Wellington, New Zealand.

Chen, C.-F. E. (2001). Making e-mail requests to professors: Taiwanese vs. American students. Paper presented at the Annual Meeting of the American Association for Applied Linguistics, St. Louis, USA.

Chen, C.-F. E. (2006). The development of e-mail literacy: From writing to peers to writing to authority figures. Language Learning \& Technology, 10(2), 35-55. 
Corder, S., \& Meyerhoff, M. (2007). Communities of practice in the analysis of intercultural communication. In H. Kotthoff \& H. Spencer-Oatey (Eds.), Handbook of intercultural communication (pp. 441-464). Berlin: Mouton de Gruyter.

Craven, A., \& Potter, J. (2010). Directives: Entitlement and contingency in action. Discourse Studies, 12(4), 419-442. doi: 10.1177/1461445610370126

Creese, A. (2010). Linguistic ethnography. In L. Litosseliti (Ed.), Rresearch methods in linguistics (pp. 138-154). London; New Yrok: Continuum.

Creswell, J. W. (2003). Research design: Qualitative, quantitative and mixed methods approaches (2nd ed.). California: Sage Publications, Inc.

Crystal, D. (2006). The language of email. In D. Crystal (Ed.), Language and the internet (2nd ed., pp. 99-133). Cambridge: Cambridge University Press.

Daft, R. L., \& Lengel, R. H. (1984). Information richness: A new approach to managerial behaviour and organizational design. In L. L. Cummings \& B. M. Staw (Eds.), Research in organizational behaviour (pp. 191-233). Greenwich, CT: JAI Press.

Daft, R. L., \& Lengel, R. H. (1986). A proposed integration among organizational information requirements, media richness, and structural design. Management Science, 32, 554571.

Daft, R. L., Lengel, R. H., \& Trevino, L. K. (1987). Message equivocality, media selection, and manager performance: Implications for information systems. MIS Quarterly, 11(3), 355-366.

Davies, B. (2005). Communities of practice: Legitimacy not choice. Journal of Sociolinguistics, 9(4), 557-581. doi: 10.1111/j.1360-6441.2005.00306.x

Davies, K. (2013, 14 March). Is being polite in email a waste of time? Calls to end perfunctory sign offs such as 'regards', UK Daily Mail. Retrieved from http://www.dailymail.co.uk/news/article-2292988/Is-time-say-Regards-email-signoffs-Debate-rages-need-polite-farewell-online-exchanges.html

Davis, K. A., \& Henze, R. C. (1998). Applying ethnographic perspectives to issues in crosscultural pragmatics. Journal of Pragmatics, 30(4), 399-419. doi: 10.1016/S03782166(98)00010-1 
December, J. (1997). Notes on defining computer-mediated communication Retrieved 27 September, 2010, from http://www.december.com/cmc/mag/1997/jan/december.html

Denscombe, M. (2010). The good research guide: For small-scale social research projects (4th ed.). Maidenhead, England: McGraw-Hill/ Open University Press.

Denzin, N. K. (1989). The research act: A theoretical introduction to sociological methods (3rd ed.). Englewood Cliffs, New Jersey: Prentice Hall.

Dornyei, Z. (2007). Research methods in applied linguistics: Quantitative, qualitative and mixed methodologies. Oxford: Oxford University Press.

Duff, P. A. (2006). Beyond generalizability: Contextualization, complexity, and credibility in applied linguistics research. In M. Chalhoub-Deville, C. A. Chapelle \& P. A. Duff (Eds.), Inference and generalizability in applied linguistics: Multiple perspectives (Vol. 12, pp. 65-95). Amsterdam; Philadelphia: John Benjamins Publishing Company.

Duff, P. A. (2010). Research approaches in applied linguistics. In R. B. Kaplan (Ed.), The Oxford handbook of applied linguistics (2nd ed., pp. 45-59). Oxford: Oxford University Press.

Eckert, P. (2000). Linguistic variation as social practice: The linguistic construction of identity in Belten High. Malden, Mass., Oxford: Blackwell.

Eckert, P., \& McConnell-Ginet, S. (1992). Think practically and look locally: Language and gender as community-based practice. Annual Review of Anthropology, 21(1), 461-490.

Eckert, P., \& McConnell-Ginet, S. (1999). New generalizations and explanations in language and gender research. Language in Society, 28(2), 185-201.

Eckert, P., \& McConnell-Ginet, S. (2003). Language and gender. Cambridge: Cambridge University Press.

Eckert, P., \& McConnell-Ginet, S. (2007). Putting communities of practice in their place. Gender and Language, 1(1), 27-37. doi: 10.1558/genl.2007.1.1.27

Eckert, P., \& Wenger, É. (2005). Communities of practice in sociolinguistics. Journal of Sociolinguistics, 9(4), 582-589. doi: 10.1111/j.1360-6441.2005.00307.x 
Economidou-Kogetsidis, M. (2005). "Yes, tell me please, what time is the midday flight from Athens arriving?": Telephone service encounters and politeness. Intercultural Pragmatics, 2(3), 253-273. doi: 10.1515/iprg.2005.2.3.253

Economidou-Kogetsidis, M. (2011). "Please answer me as soon as possible": Pragmatic failure in non-native speakers' e-mail requests to faculty. Journal of Pragmatics, 43(13), 3193-3215. doi: 10.1016/j.pragma.2011.06.006

Eelen, G. (2001). A critique of politeness theory. Manchester: St. Jerome.

Emerson, R. M., Fretz, R. I., \& Shaw, L. L. (2001). Participant observation and fieldnotes. In P. Atkinson, A. Coffey, S. Delamont, J. Lofland \& L. H. Lofland (Eds.), Handbook of ethnography (pp. 352-368). London, UK: SAGE Publications Ltd.

Ervin-Tripp, S. (1976). Is Sybil there? The structure of some American English directives. Language in Society, 5(1), 25-66.

Feagin, C. (2013). Entering the community: Fieldwork J. K. Chambers \& N. Schilling-Estes (Eds.), The handbook of language variation and change (pp. 19-37). Retrieved from http://site.ebrary.com.helicon.vuw.ac.nz/lib/vuw/docDetail.action?doclD=10777211

Ferrara, K., Brunner, H., \& Whittemore, G. (1991). Interactive written discourse as an emergent register. Written Communication, 8(1), 8-34. doi: $10.1177 / 0741088391008001002$

File, K. A. (2013). The post-match interview as a discourse genre: A combined genre and register analysis. Unpublished PhD thesis. Victoria University of Wellington, Wellington, New Zealand. Retrieved from http://hdl.handle.net/123456789/8563

Fletcher, J. R. (2011). The role of discourse in establishing an enabling context for organizational knowledge creation: An ethnographic study. Unpublished PhD thesis. Victoria University of Wellington, Wellington, New Zealand.

French, J. R. P. J., \& Raven, J. (1968). The bases of social power. In D. Cartwright \& A. Zander (Eds.), Group dynamics: Research and theory (3rd ed., pp. 259 - 277). London, UK: Tavistock Publications Limited.

Frey, L. R., Botan, C. H., Friedman, P. G., \& Kreps, G. L. (1991). Investigating communication: An introduction to research methods. New Jersey: Prentice Hall. 
Gains, J. (1999). Electronic mail - a new style of communication or just a new medium?: An investigation into the text features of email. English for Specific Purposes, 18(1), 81101. doi: 0889-4906/98 $\$ 19.00+0.00$

Gillaerts, P. (2012). E-mail use in a Belgian company: Looking for the hybridity of the genre. In P. Gillaerts, E. de Groot, S. Dieltjens, P. Heynderickx \& G. Jacobs (Eds.), Researching discourse in business genres: Cases and corpora (Vol. 152, pp. 15-31). Bern, Switzerland: Peter Lang AG.

Gimenez, J. (2000). Business e-mail communication: Some emerging tendencies in register. English for Specific Purposes, 19(3), 237-251. doi: 10.1016/S0889-4906(98)00030-1

Goffman, E. (1967). Interaction ritual: Essays on face to face behaviour. New York: Anchor Books.

Green, G. (1975). How to get people to do things with words: The whimperative question. In P. Cole \& J. L. Morgan (Eds.), Syntax and semantics 3: Speech acts (pp. 107-142). New York: Academic Press.

Gu, Y. (1990). Politeness phenomena in modern Chinese. Journal of Pragmatics, 14(2), $237-$ 257. doi: http://dx.doi.org/10.1016/0378-2166(90)90082-O

Halliday, M. A. K. (1973). Explorations in the functions of language. New York: Elsevier NorthHolland, Inc.

Halliday, M. A. K. (1985). An introduction to functional grammar London: Edward Arnold (Publishers) Ltd.

Halliday, M. A. K. (1994). An introduction to functional grammar (2nd ed.). London: Edward Arnold.

Halliday, M. A. K. (2009). The essential Halliday. London; New York: Continuum International Publishing Group.

Halliday, M. A. K., \& Matthiessen, C. M. I. M. (2004). An introduction to functional grammar (3rd ed.). London: Arnold.

Hammersley, M. (2007). Reflections on linguistic ethnography. Journal of Sociolinguistics, 11(5), 689-695. doi: 10.1111/j.1467-9841.2007.00347.x 
Hammersley, M., \& Atkinson, P. (2007). Ethnography: Principles in practice (3rd ed.). New York: Routledge.

Harrison, S. (1998). Email discussions as conversation: Move and acts in a sample from a listserv discussion. Linguistics Online, 1(1), 98.

Hartford, B. S., \& Bardovi-Harlig, K. (1996). "At your earliest convenience": A study of written student requests to faculty. In L. Bouton (Ed.), Pragmatics and language learning (pp. 55-69). Urbana-Campaign, Illinois: University of Illinois.

Haugh, M. (2003). Japanese and non-Japanese perceptions of Japanese communication. New Zealand Journal of Asian Studies, 5(1), 156-177.

Haugh, M. (2004). Revisiting the conceptualisation of politeness in English and Japanese. Multilingua, 23(1/2), 85-109.

Haugh, M. (2007). The discursive challenge to politeness research: An interactional alternative. Journal of Politeness Research: Language, Behavior, Culture, 3(2), 295317. doi: $10.1515 / p r .2007 .013$

Haugh, M. (2010). When is an email really offensive?: Argumentativity and variability in evaluations of impoliteness. Journal of Politeness Research: Language, Behavior, Culture, 6(1), 7-31. doi: 10.1515/jplr.2010.002

Hay, J. (2001). The pragmatics of humor support. Humor: International Journal of Humor Research, 14(1), 55.

Hendriks, B. (2010). An experimental study of native speaker perceptions of non-native request modification in e-mails in English. Intercultural Pragmatics, 7(2), 221-255. doi: 10.1515/iprg.2010.011

Herring, S. (2001). Computer mediated discourse. In D. Schiffrin, D. Tannen \& H. E. Hamilton (Eds.), The handbook of discourse analysis. Oxford: Blackwell Publishers Ltd.

Hiemstra, G. E. (1986). The electronic organisation: Communication and organising in a new age. In L. Thayer (Ed.), Organisation communication: Emerging perspectives (pp. 196220). Norwood, NJ: Ablex. 
Ho, C. K. V. (2009). A multi-perspective analysis of the request e-mail discourse of a team of education professionals in Hong Kong. Unpublished PhD thesis. Macquarie University, Australia

Holmes, J. (1983). The structure of teachers' directives. In J. C. Richards \& R. W. Schmidt (Eds.), Language and communication (pp. 89-115). London: Longman.

Holmes, J. (1995). Women, men and politeness. London; New York: Longman.

Holmes, J. (1999). Managing in style: Flexible discourse at work. ERIC Document 427525.

Holmes, J. (2000a). Politeness, power and provocation: How humour functions in the workplace. Discourse Studies, 2(2), 159-185. doi: 10.1177/1461445600002002002

Holmes, J. (2000b). Victoria University of Wellington's language in the workplace project: An overview. Language in the Workplace Occasional Papers(1), 1-18.

Holmes, J. (2005). Story-telling at work: A complex discursive resource for integrating personal, professional and social identities. Discourse Studies, 7(6), 671-700. doi: $10.1177 / 1461445605055422$

Holmes, J. (2006). Sharing a laugh: Pragmatic aspects of humor and gender in the workplace. Journal of Pragmatics, 38(1), 26-50. doi: 10.1016/j.pragma.2005.06.007

Holmes, J., \& Marra, M. (2002). Having a laugh at work: How humour contributes to workplace culture. Journal of Pragmatics, 34(12), 1683-1710. doi: 10.1016/S03782166(02)00032-2

Holmes, J., Marra, M., \& Vine, B. (2011). Leadership, discourse and ethnicity. Oxford, UK: Oxford University Press.

Holmes, J., Marra, M., \& Vine, B. (2012). Politeness and impoliteness in ethnic varieties of New Zealand English. Journal of Pragmatics, 44(9), 1063-1076. doi: http://dx.doi.org/10.1016/j.pragma.2011.11.006

Holmes, J., \& Meyerhoff, M. (1999). The community of practice: Theories and methodologies in language and gender research. Language in Society, 28(2), 173-183. 
Holmes, J., \& Stubbe, M. (2003a). "Feminine" workplaces: Stereotype and reality. In J. Holmes \& M. Meyerhoff (Eds.), The handbook of language and gender (pp. 573-599). Malden, MA: Blackwell Publishing Ltd.

Holmes, J., \& Stubbe, M. (2003b). Power and politeness in the workplace: A sociolinguistic analysis of talk at work. London: Longman.

Holmes, J., Stubbe, M., \& Vine, B. (1999). Constructing professional identity: "Doing power" in policy units. In S. Sarangi \& C. Roberts (Eds.), Talk, work and institutional order: Discourse in medical, mediation and management settings (pp. 351-385). Berlin; New York: Mouton de Gruyter.

House, J. (1989). Politeness in English and German: The functions of please and bitte. In B.-K. Shoshana, J. House \& G. Kasper (Eds.), Cross-cultural pragmatics: Requests and apologies (Vol. XXXI, pp. 96-119). Norwood, New Jersey: Ablex Publishing Corporation.

House, J., \& Kasper, G. (1980). Politeness markers in English and German. Journal of Pragmatics, 4(2), 157 - 185.

Huang, W., Watson, R. T., \& Wei, K. K. (1998). Can a lean email medium be used for rich communication? A psychological perspective. European Journal of Information Systems, 7(4), 269-274.

Hyland, K. (2005). Metadiscourse: Exploring interaction in writing. London: Continuum.

Hymes, D. (1968). The ethnography of speaking. In J. A. Fishman (Ed.), Readings in the sociology of language. The Hague: Mouton.

Hymes, D. (1970). Linguistic aspects of comparative political research. In R. Holt \& J. Turner (Eds.), The methodology of comparative research (pp. 295-341). New York: The Free Press.

Hymes, D. (1974). Foundations in sociolingistics: An ethnographic approach. Philadelphia: University of Pennsylvania Press.

Hymes, D. (1996). Ethnography, linguistics, narrative inequality: Toward an understanding of voice. London: Taylor and Francis. 
Ide, S. (1989). Formal forms and discernment: Two neglected aspects of universals of politeness. Multilingua, 8(2-3), 223-248.

Jensen, A. (2009). Discourse strategies in professional email negotiation: A case study. English for Specific Purposes, 28(1), 4-18.

Jewson, N. (2007). Communities of practice in their place: Some implicaitons of changes in the spatial location of work. In J. Hughes, N. Jewson \& L. Unwin (Eds.), Communities of practice: Critical perspectives. London; New York: Routledge.

Johnston, L., \& Robertson, S. (1993). "Hey yous!" the Maori-NZE interface in sociolinguistic rules of address. Te Reo, 36, 115 - 127.

Jones, J. S. (2010). Introductions. In J. S. Jones \& S. Watt (Eds.), Ethnography in social science practice (pp. 3-12). Abingdon, Oxon: Routledge.

Kankaanranta, A. (2005). "Hej Seppo, could you pls comment on this!": Internal email communication in lingua franca English in a multinational company. Unpublished $\mathrm{PhD}$ Thesis. University of Jyväskylä, Finland. Retrieved from http://urn.fi/URN:ISBN:95139-2320-7

Kiesler, S., Siegel, J., \& McGuire, T. W. (1984). Social psychological aspects of computermediated communication. American Psychologist, 39(10), 1123-1134. doi: 10.1037/0022-3514.36.1.110.1037/0003-066x.39.10.1123

King, B. W. (2011). Linguistic negotiations of sexual agency in sexuality education. Unpublished PhD thesis. Victoria University of Wellington, Wellington, New Zealand.

King, J. (1999). Talking bro: Māori English in the university setting. Te Reo, 42, 20-38.

$\mathrm{Ku}, \mathrm{L}$. (1996). Social and non-social uses of electronic messaging systems in organisation. Journal Business Communication, 33(3), 297-325.

Ladegaard, H. J. (2009). Politeness, power and control: The use of humour in cross-cultural telecommunications. In W. Cheng \& K. C. C. Kong (Eds.), Professional communication: Collaboration between academics and practitioners (pp. 191-209). Hong Kong: Hong Kong University Press. 
Ladegaard, H. J. (2011). 'Doing power' at work: Responding to male and female management styles in a global business corporation. Journal of Pragmatics, 43(1), 4-19. doi: http://dx.doi.org/10.1016/j.pragma.2010.09.006

Lakoff, R. (1973). The logic of politeness: or, Minding your P's and Q's. Chicago Linguistic Society, 9, 292-305.

Lan, L. (2000). Email: A challenge to standard English. English Today, 16(4), 23-29. doi: $10.1017 /$ S0266078400000511

Larson-Hall, J. (2010). A guide to doing statistics in second language research using SPSS. New York; London: Routledge.

Lave, J., \& Wenger, E. (1991). Situated Learning: Legitimate peripheral participation. Cambridge; New York: Cambridge University Press.

Laver, J. (1975). Communicative functions of phatic communion. In A. Kendon, R. M. Larris \& M. R. Key (Eds.), Organisation of behaviour in face-to-face interaction (pp. 215-238). The Hague: Mouton Publishers.

Lee-Wong, S. M. (1994a). Politeness and face in Chinese culture (Vol. 6). Frankfurt; Berlin; Bern; Bruxelles; New York; Wien: Peter Lang.

Lee-Wong, S. M. (1994b). Qing/please - a polite or requestive marker?: Observations from Chinese. Multilingua, 13(4), 343-360. doi: 156.62.3.11

Lee, C. L. (2011). Politeness in Singapore. In D. Z. Kadar \& S. Mills (Eds.), Politeness in East Asia (pp. 226 - 251). Cambridge: Cambridge University Press.

Leech, G. (1983). Principles of pragmatics. London; New York: Longman.

Leech, G. (1999). The distribution and function of vocatives in American and British English conversation. In S. J. Johansson, H. Hasselgard \& S. Oksefjell (Eds.), Out of corpora: Studies in honour of Stig Johansson (pp. 107 - 118). Amsterdam: Rodopi.

Leech, G. (2007). Politeness: Is there an East-West divide? Journal of Politeness Research: Language, Behavior, Culture, 3(2), 167-206. doi: 10.1515/pr.2007.009

Levinson, S. C. (1983). Pragmatics. Cambridge: Cambridge University Press. 
Li, L. (2000a). Email: A challenge to standard English. English Today 64, 16(4), 23 - 55.

Li, Y. (2000b). Surfing e-mails. English Today, 16(4), 30-34. doi: 10.1017/S0266078400000523

Locher, M. A. (2004). Power and politeness in action: Disagreements in oral communication. Berlin; New York: Mouton de Gruyter.

Locher, M. A., \& Watts, R. J. (2005). Politeness theory and relational work. Journal of Politeness Research: Language, Behaviour and Culture, 1(1), 9-33. doi: AN 16634946

Lofland, J., \& Lofland, L. H. (1995). Analyzing social settings (A guide to qualitative observation and analysis) (3rd ed.). California: Wadsworth Publishing Company.

Long, R. J. (1987). New office information technology: Human and managerial implications. London; New York: Croom Helm.

Louhiala-Salminen, L. (1996). The business communication classroom vs reality: What should we teach today? English for Specific Purposes, 15(1), 37-51. doi: 10.1016/08894906(95)00024-0

Louhiala-Salminen, L., Charles, M., \& Kankaanranta, A. (2005). English as a lingua franca in Nordic corporate mergers: Two case companies. English for Specific Purposes, 24(4), 401-421. doi: 10.1016/j.esp.2005.02.003

Macksoud, R. (2010). Using interview data in case studies. In S. Hunston \& D. Oakey (Eds.), Introducing applied linguistics: Concepts and skills (pp. 153-159). Abingdon, Oxon: Routledge.

Malinowski, B. (1992). Argonauts of the Western Pacific. London: Routledge and Kegan Paul.

Maricic, I. (2005). Face in cyberspace : Facework, (im)politeness and conflict in English discussion groups. Växjö: Växjö University Press.

Markus, L. M. (1994a). Electronic mail as a medium of managerial choice. Organization Science, 5(4), 502-527. doi: 1047-7039/94/0504/0502/\$01.25 
Markus, L. M. (1994b). Finding a happy medium: Explaining the negative effects of electronic communication on social life at work. ACM Transactions on Information Systems, 12(2), 119-149. doi: 10.1145/196734.196738

Marra, M. (2003). Decisions in New Zealand business meetings: A sociolinguistic analysis of power at work. Unpublished PhD thesis. Victoria University of Wellington, Wellington, New Zealand.

Marra, M. (2008). Recording and analysing talk across culture. In H. Spencer-Oatey (Ed.), Culturally speaking: Managing rapport through talk across cultures (pp. 304-321). London: Continuum.

Marra, M., \& Holmes, J. (2007). Humour across culture: Joking in the multiculture workplace. In H. Kotthoff \& H. Spencer-Oatey (Eds.), Handbook of intercultural communication (pp. 153-172). Berlin: Mouton de Gruyter.

Marra, M., \& Holmes, J. (2008). Constructing ethnicity in New Zealand workplace stories. Text \& Talk, 28(3), 397-419. doi: 10.1515/text.2008.019

Matsumoto, Y. (1988). Reexamination of the universality of face: Politeness phenomena in Japanese. Journal of Pragmatics, 12(4), 403-426. doi: 10.1016/0378-2166(88)90003-3

McConnell-Ginet, S. (2003). "What's in a name?" Social labeling and gender practices. In J. Holmes \& M. Meyerhoff (Eds.), The Handbook of Language and Gender. Oxford, UK: Blackwell Publishing Ltd.

Mendoza-Denton, N. (2008). Homegirls: Language and cultural practice among Latina youth gangs (Vol. New Directions in Ethnography). Malden, MA: Blackwell Publication.

Miller, K. (2009). Organizational communication: Approaches and processes (5th ed.). Boston, MA: Wadsworth Cengage Learning.

Mills, J. E., Clay, J. M., \& Mortensen, M. (2000). You've Got Trouble!: Managin E-mail Liability. Cornell Hotel and Restaurant Administration Quarterly, 41(5), 64-71. doi: 10.1177/001088040004100536

Mills, S. (2003). Gender and politeness. Cambridge: Cambridge University Press.

Milroy, L. (1987). Language and social networks (2nd ed.). Oxford, UK; New York: Blackwell. 
Morand, D. A. (1996). What's in a name?: An exploration of the social dynamics of forms of address in organizations. Management Communication Quarterly, 9(4), 422-451. doi: $10.1177 / 0893318996009004003$

Morand, D. A., \& Ocker, R. J. (2003). Politeness theory and computer-mediated communication: A sociolinguistic approach to analyzing relational messages. Paper presented at the 36th Hawaii International Conference on System Sciences, Hawaii.

Morpeth, N. V. D. E. (2012). Politeness and gender in Belgian organisational emails. In P. Gillaerts, E. de Groot, S. Dieltjens, P. Heynderickx \& G. Jacobs (Eds.), Researching discourse in business genres: Cases and corpora (Vol. 152, pp. 33-51). Bern, Switzerland: Peter Lang AG.

Mumby, D. K. (1988). Communication and power in organizations: Discourse, ideology and domination. Norwood, NJ: Ablex.

Murata, K. (2011). Relational practice in meeting discourse in New Zealand and Japan :A cross-cultural study. Unpublished PhD thesis. Victoria University of Wellington, Wellington, New Zealand. Retrieved from http://restrictedarchive.vuw.ac.nz/bitstream/handle/123456789/5416/thesis.pdf?se quence $=2$

Murray, D. E. (1988). Computer-mediated communication: Implications for ESP. English for Specific Purposes, 7(1), 3-18. doi: 10.1016/0889-4906(88)90003-8

Nadler, J., \& Shestowsky, D. (2006). Negotiation, information technology, and the problem of the faceless other. In L. L. Thompson (Ed.), Negotiation theory and research (pp. 145172). New York: Psychology Press.

Ngwenyama, O. K., \& Lee, A. S. (1997). Communication richness in electronic mail: Critical social theory and the contextuality of meaning. MIS Quarterly, 21(2), 145-167.

Nickerson, C. (1999). The use of English in electronic mail in a multinational corporation. In F. Bargiela-Chiappini \& C. Nickerson (Eds.), Genres, media and discourses (pp. 35-56). Essex, UK: Longman.

Nunan, D. (1992). Research methods in language learning. Cambridge, UK: Cambridge University Press. 
Orlikowski, W. J., \& Yates, J. (1994). Genre repertoire: The structuring of communicative practices in organizations. Administrative Science Quarterly, 39(4), 541-574.

Palmer, C. (2010). Observing with a focus: Field notes and data recording. In J. S. Jones \& S. Watt (Eds.), Ethnography in social science practice (pp. 141-156). Abingdon, Oxon: Routledge.

Pan, Y. (1995). Power behind linguistic behavior: Analysis of politeness phenomena in Chinese official settings. Journal of Language and Social Psychology, 14(4), 462-481. doi: 10.1177/0261927x950144007

Panteli, N., \& Seeley, M. (2006). The email gender gap. In M. Barrett, and Davidson, Marilyn J. (Ed.), Gender and communication at work (pp. 250-259). Hampshire: Ashgate Publishing Limited.

Paramasivam, S. (2007). Managing disagreement while managing not to disagree: Polite disagreement in negotiation discourse. Journal of Intercultural Communication Research, 36(2), 91-116. doi: 10.1080/17475750701478661

Paramasivam, S. (2011). Rapport management in air traffic control in Malaysian aviation discourse. Journal of Asian Pacific Communication, 21(1), 77-96.

Parry, R. (2013). Giving reasons for doing something now or at some other time. Research on Language and Social Interaction, 46(2), 105-124. doi: 10.1080/08351813.2012.754653

Planken, B. (2005). Managing rapport in lingua franca sales negotiations: A comparison of professional and aspiring negotiators. English for Specific Purposes, 24(4), 381-400. doi: http://dx.doi.org/10.1016/i.esp.2005.02.002

Radicati, S. (2001). Email statistics report, 2001 - 2005 Retrieved 18 July 2011, from http://findarticles.com/p/articles/mi_m4422/is 5 18/ai_78404402/?tag=mantle ski $\underline{\mathrm{n} \text {;content }}$

Radicati, S. (2010). Email statistics report, 2010 - 2014 Retrieved 18 July 2011, April 2010, from http://www.radicati.com/wp/wp-content/uploads/2010/04/Email-StatisticsReport-2010-2014-Executive-Summary2.pdf 
Radicati, S. (2012). Email statistics report, 2012 - 2016 Retrieved 11 May, 2012, from http://www.radicati.com/wp/wp-content/uploads/2012/04/Email-Statistics-Report2012-2016-Executive-Summary.pdf

Ramli, R. (2013). Culturally appropriate communication in Malaysia: Budi bahasa as warranty component in Malaysian discourse. Journal of Multicultural Discourses, 8(1), 65-78. doi: 10.1080/17447143.2012.753895

Rampton, B. (2007). No-Hymesian linguistic ethnography in the United Kingdom. Journal of Sociolinguistics, 11(5), 584-607.

Rice, R. E., \& Love, G. (1987). Electronic emotion. Communication Research, 14(1), 85-108. doi: $10.1177 / 009365087014001005$

Rice, R. P. (1997). An analysis of stylistic variables in electronic mail. Journal of Business and Technical Communication, 11(5), 5-23. doi: 10.1177/1050651997011001001

Rock, F. (2005). "I've picked some up from a colleague": Language, sharing and communities of practice in an institutional setting. In D. Barton \& K. Tusting (Eds.), Beyond communities of practice: Language, power, and social context (pp. 77 - 104). Cambridge, UK: Cambridge University Press.

Sato, S. (2008). Use of "please" in American and New Zealand English. Journal of Pragmatics, 40(7), 1249-1278. doi: 10.1016/j.pragma.2007.09.001

Saville-Troike, M. (1997). Ethnographic analysis of communicative events. In N. Coupland \& A. Jaworski (Eds.), Sociolinguistics: A reader and coursebook (pp. 126-144). New York: Palgrave.

Saville-Troike, M. (2003). The ethnography of communication: An introduction (3rd ed.). Malden, MA: Blackwell Publication.

Schegloff, E. A. (1986). The routine as achievement. Human Studies, 9(2/3), 111-151.

Schein, E. H. (1990). Organizational culture. American Psychologist, 45(2), 109-119. doi: 10.1016/0090-2616(83)90023-2

Schnurr, S. (2005). Humour and leadership discourse in different workplace cultures. Unpublished PhD thesis. Victoria University of Wellington, Wellington, New Zealand. Retrieved from http://restrictedarchive.vuw.ac.nz/handle/123456789/1265 
Schnurr, S. (2009). Constructing leader identities through teasing at work. Journal of Pragmatics, 41(6), 1125-1138. doi: http://dx.doi.org/10.1016/j.pragma.2008.10.002

Schnurr, S., \& Chan, A. (2009). Politeness and leadership discourse in New Zealand and Hong Kong: A cross-cultural case study of workplace talk. Journal of Politeness Research, 5(2), 131-157. doi: 10.1515/JPLR.2009.009

Schnurr, S., \& Chan, A. (2011). When laughter is not enough. Responding to teasing and selfdenigrating humour at work. Journal of Pragmatics, 43(1), 20-35. doi: http://dx.doi.org/10.1016/j.pragma.2010.09.001

Schnurr, S., Marra, M., \& Holmes, J. (2007). Being (im)polite in New Zealand workplaces: Māori and Pākehā leaders. Journal of Pragmatics, 39(4), 712-729. doi: 10.1016/j.pragma.2006.11.016

Scollon, R., \& Wong Scollon, S. (2001). Intercultural communication: A discourse approach (2nd ed.). Cambridge, Mass and Oxford: Blackwell.

Searle, G. J. (2003). Workplace communications: The basics (2nd ed.). New York: Longman.

Searle, J. R. (1969). Speech acts. Cambridge: Cambridge University Press.

Searle, J. R. (1976). A classification of illocutionary acts. Language in Society, 5(1), 1-23.

Sherblom, J. (1988). Direction, function, and signature in electronic mail. Journal of Business Communication, 25(4), 39-54.

Sinclair, J. M., \& Coulthard, R. M. (1975). Towards an analysis of discourse: The English used by teachers and pupils London: Oxford University Press.

Smircich, L. (1983). Concepts of culture and organizational analysis. Administrative Science Quarterly, 28(3), 339-358.

Spencer-Oatey, H. (1993). Conceptions of social relations and pragmatics research. Journal of Pragmatics, 20(1), 27-47. doi: http://dx.doi.org/10.1016/0378-2166(93)90105-X

Spencer-Oatey, H. (2000a). Culturally speaking: Managing rapport in talk across cultures London: Cassell. 
Spencer-Oatey, H. (2000b). Introduction: Language, culture and rapport management. In H. Spencer-Oatey (Ed.), Culturally speaking: Managing rapport through talk across cultures (pp. 1-8). London: Continuum.

Spencer-Oatey, H. (2000c). Rapport management: A framework for analysis. In H. SpencerOatey (Ed.), Culturally speaking: Managing rapport through talk across cultures (pp. 11-46). London: Continuum.

Spencer-Oatey, H. (2005a). (Im)politeness, face and perceptions of rapport: Unpackaging their bases and interrelationships. Journal of Politeness Research, 1(1), 95-119.

Spencer-Oatey, H. (2005b). Rapport management theory and culture. Intercultural Pragmatics, 2(3), 335-346. doi: 10.1515/iprg.2005.2.3.335.

Spencer-Oatey, H. (2007). Theories of identity and the analysis of face. Journal of Pragmatics, 39(4), 639-656. doi: 10.1016/j.pragma.2006.12.004

Spencer-Oatey, H. (Ed.). (2008). Culturally speaking: Culture, communication and politeness theory (2nd ed.). London; New York: Continuum.

Spencer-Oatey, H., \& Franklin, P. (2009). Intercultural Interaction: A multidisciplinary approach to intercultural communication. Basingstoke: Palgrave Macmillan.

Spencer-Oatey, H., \& Jiang, W. (2003). Explaining cross-cultural pragmatic findings: Moving from politeness maxims to sociopragmatic interactional principles (SIPs). Journal of Pragmatics, 35(10-11), 1633-1650. doi: 10.1016/S0378-2166(03)00025-0

Spencer-Oatey, H., \& Xing, J. (2000). A problematic Chinese business visit to Britain: Issues of face. In H. Spencer-Oatey (Ed.), Culturally speaking: Managing rapport through talk across cultures. London and New York: Continuum.

Spencer-Oatey, H., \& Xing, J. (2003). Managing rapport in intercultural business interactions: A comparison of two Chinese-British welcome meetings. Journal of Intercultural Studies, 24(1), 33-46. doi: 10.1080/0725686032000115374

Spencer-Oatey, H., \& Xing, J. (2008). Issues of face in a Chinese business visit to Britain. In H. Spencer-Oatey (Ed.), Culturally speaking: Culture, communication and politeness theory (pp. 258-273). London; New York: Continuum International Publishing Group. 
Sproull, L., \& Kiesler, S. (1986). Reducing social context cues: Electronic mail in organisation. Management Science, 32(11), 1492-1513. doi: 0025-1909/86/3211/1492S01.25

Starfield, S. (2010). Ethnographies. In B. Paltridge \& A. Phakiti (Eds.), Continuum companion to research methods in applied linguistics (pp. 50-65). London: Continuum.

Stubbs, M. (1983). Discourse analysis: The sociolinguistic analysis of natural language and culture. Oxford: Blackwell.

Sullivan, C. B. (1995). Preferences for electronic mail in organizational communication tasks. The Journal of Business Communication, 32, 49-64.

Tagg, C. (2012). Discourse of text messaging: Analysis of text message communication. London, New York: Continuum International Publishing Group.

Tanaka, N., Spencer-Oatey, H., \& Cray, E. (2008). Apologies in Japanese and English. In H. Spencer-Oatey (Ed.), Culturally speaking: Culture, communication and politeness theory (pp. 73-94). London: Continuum.

Tannen, D. (1993). What's in a frame?: Surface evidence for underlying expectations. In D. Tannen (Ed.), Framing in discourse (pp. 14-56). Oxford: Oxford University Press.

Terkourafi, M. (2005). Beyond the micro-level in politeness research. Journal of Politeness Research: Language, Behavior, Culture, 1(2), 237-262. doi: 10.5555/jplr.2005.1.2.237

Thomas, J. (1995). Meaning in interaction: An introduction to pragmatics. New York: Longman Group Limited.

Thomson, R. (2006). Gender and electronic discourse in the workplace. In M. Barrett, and Davidson, Marilyn J. (Ed.), Gender and communication at work (pp. 239-249). Hampshire: Ashgate Publishing Limited.

Ting-Toomey, S., \& Kurogi, A. (1998). Facework competence in intercultural conflict: An updated face-negotiation theory. International Journal of Intercultural Relations, 22(2), 187-225. doi: 10.1016/S0147-1767(98)00004-2

Trevor-Roberts, E., Ashkanasy, N. M., \& Kennedy, J. C. (2003). The egalitarian leader: A comparison of Leadership in Australia and New Zealand. Asia Pacific Journal of Management, 20(4), 517-540. 
Usó-Juan, E., \& Martínez-Flor, A. (2008). Teaching learners to appropriately mitigate requests. ELT Journal, 62(4), 349-357. doi: 10.1093/elt/ccm092

Van Mulken, M., \& Van der Meer, W. (2005). Are you being served? A genre analysis of American and Dutch company replies to customer enquiries. English for Specific Purposes, 24(1), 93-109. doi: 10.1016/j.esp.2004.07.001

Vine, B. (2001). Workplace language and power: Directives, requests and advice. Unpublished PhD thesis. Victoria University of Wellington, Wellington, New Zealand.

Vine, B. (2009). Directives at work: Exploring the contextual complexity of workplace directives. Journal of Pragmatics, 41(7), 1395-1405. doi: http://dx.doi.org/10.1016/j.pragma.2009.03.001

Wagner, E. (2010). Survey research. In B. Paltridge \& A. Phakiti (Eds.), Continuum companion to research methods in applied linguistics. London; New York: Continuum International Publishing Group.

Waldvogel, J. (1999). Email: User perceptions and aspects of its role and impact in one Wellington workplace. Unpublished MA research project. Victoria University of Wellington, Wellington, New Zealand.

Waldvogel, J. (2001). Email and workplace communication: A literature review. Language in the Workplace Occasional Papers(3).

Waldvogel, J. (2005). The role, status and style of workplace email: A study of two New Zealand workplaces. Unpublished PhD thesis. Victoria University of Wellington, Wellington, New Zealand.

Waldvogel, J. (2007). Greetings and closings in workplace email. Journal of ComputerMediated Communication, 12(2), 456-477. doi: 10.1111/j.1083-6101.2007.00333.x

Watt, S., \& Jones, J. S. (2010). Let's look inside: Doing participant observation. In S. Watt \& J. S. Jones (Eds.), Ethnography in social science practice (pp. 107-125). Abingdon, Oxon: Routledge.

Watts, R. (2003). Politeness. Cambridge: Cambridge University Press. 
Watts, R. (2005). Linguistic politeness and politic behaviour: Reconsidering claims for universality. In R. Watts, I. Sachiko \& K. Ehlich (Eds.), Politeness in language: Studies in its history, theory, and practice (pp. 43 - 71). Berlin; New York: Mouton de Gruyter.

Webb, E. J., Campbell, D. T., Schwartz, R. D., \& Sechrest, L. (1966). Unobstrusive measures: Nonreactive measures in the social sciences. Chicago: Rand McNally.

Wenger, E. (1998a). Communities of practice. Cambridge \& New York: Cambridge University Press.

Wenger, E. (1998b). Communities of practice: Learning as a social system. Systems Thinker, 9(5), 1-5.

Wenger, E., McDermott, R., \& Snyder, W. M. (2002). Cultivating communities of practice: A guide to managing knowledge. Boston, MA: Harvard Business School Press.

Wenger, E., \& Snyder, W. M. (2000). Communities of practice: The organizational frontier. Harvard Business Review, 78(1), 139-145.

Wierzbicka, A. (2003). Cross-cultural pragmatics: The semantics of human interaction (2nd ed.). Berlin: Mouton de Gruyter.

Willis, P. (2000). The ethnographic imagination. Cambridge: Polity.

Wilson, N. A. (2010). Bros, boys and guys: Address term function and communities of practice in a New Zealand rugby team. New Zealand English Journal (24), 33-54.

Wilson, N. A. (2011). Leadership as communicative practice: The discursive construction of leadership and team identity in a New Zealand rugby team. Unpublished PhD thesis. Victoria University of Wellington, Wellington, New Zealand.

Wodak, R., \& Krzyżanowski, M. (2008). Qualitative discourse analysis in the social sciences. Basingstoke [England]; New York: Palgrave Macmillan.

Wood, L. A., \& Kroger, R. O. (1991). Politeness and forms of address. Journal of Language and Social Psychology, 10(3), 145-168. doi: 10.1177/0261927X91103001 
Wootton, A. (1984). Some aspects of children's use of 'please' in request sequences. In P. Auer \& A. Di Luzio (Eds.), Interpretive sociolinguistics: Migrants, children, migrant children (pp. 147-162). Germany: Gunter Narr Verlag, Tu“bingen.

Yates, J., \& Orlikowski, W. J. (1992). Genres of organizational communication: A structurational approach to studying communication and media. Academy of Management Review, 17(2), 299-326.

Yli-Jokipii, H. (1994). Requests in professional discourse: A cross-cultural study of British, American, and Finnish business writing. Helsinki: Suomalainen Tiedeakatemia 
MEMORANDUM

\begin{tabular}{l|l}
\hline TO & Jackie Yeoh \\
\hline COPY TO & $\begin{array}{l}\text { Prof Janet Holmes } \\
\text { Dr Elaine Vine }\end{array}$ \\
\hline FROM & Dr Allison Kirkman, Convener, Human Ethics Committee \\
\hline
\end{tabular}

\begin{tabular}{l|l}
\hline DATE & 23 December 2010 \\
\hline PAGES & 1 \\
\hline
\end{tabular}

\section{Ethics Approval: No 18193 / Email communication in the} workplace

Thank you for your application for ethical approval, which has now been considered by the Standing Committee of the Human Ethics Committee.

Your application has been approved from the above date and this approval continues until 30 June 2013. If your data collection is not completed by this date you should apply to the Human Ethics Committee for an extension to this approval.

Best wishes with the research.

Allison Kirkman

Human Ethics Committee 


\section{Appendix 2: Information sheet for participants}

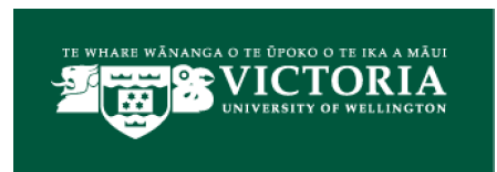

\section{Information Sheet for a Study on Email Communication in the Workplace}

Researcher: Jackie Yeoh, School of Linguistics and Applied Languages Studies, Victoria University of Wellington

\section{$\underline{\text { Introduction }}$}

I am a PhD student undertaking research on email communication in the workplace. This study will investigate the role of email in the daily communication practices in the organisation. I am therefore seeking permission to collect emails, to record and to analyse interviews. This research is part of the Wellington Language in the Workplace Project.

It is hoped that the results of this study will be useful to organisations in maintaining and improving workplace communication, in particular email communication. This study has been given ethics approval by the university's Ethics Committee. I will be seeking your written consent to take part in this study.

\section{The study}

Participation in this study is voluntary. After the email data has been collected, I will interview you about your email practices. These interviews will be audio recorded. You may withdraw from the research up to 2 months after you provide data. If you choose to withdraw, the material you have provided will be destroyed.

\section{Confidentiality}

Upon completion of the project, all the data collected will be archived in the Wellington Language in the Workplace Project. Access to this material is restricted to approved researchers. All data and notes, both written and audio-recorded will be accessible only to the researcher and the supervisors during the duration of the research and will be securely stored at all times. In any conference presentations, written publications and in the thesis, the identity of the organisation and participants will be fully protected. Any sensitive information (in the email messages) will be strictly confidential and will not be traceable to the organisation or participants.

\section{$\underline{\text { Contact Details }}$}

If you have any questions or would like to have more information, please contact me at jackie.yeoh@vuw.ac.nz or my supervisors, Professor Janet Holmes (janet.holmes@vuw.ac.nz) and Dr. Elaine Vine (elaine.vine@vuw.ac.nz) at the School of Linguistics and Applied Languages Studies, Victoria University of Wellington. They can also be contacted at 044635600. 


\title{
Language in the Workplace Project at the XXX \\ Proposal for a Cooperative Research Project
}

\begin{abstract}
About the project
Researchers from the Language in the Workplace Project have been studying workplace communication since 1996. So far, we have collected hundreds of recordings from office workers in government departments and from teams in companies such as Mobil and Telecom, from factory workers in Wellington and Auckland, and from various small businesses in Hawkes Bay and Wellington. We have analysed a number of aspects of workplace talk including how people use talk to get things done at work, how people prevent or fix up misunderstandings, and how they use humour and small talk to get on better with their workmates. In our latest project we aim to focus on email which has become a very important component in workplace communication.
\end{abstract}

\section{What will the researchers do?}

We aim to investigate the role of email in the daily communication practices in organisations. In order to achieve this, we will

(i) ask as many people as possible to complete a questionnaire on their email usage

(ii) ask some key participants to allow us to analyse a sample of their email (eg. 50-100 emails per person)

(iii) interview the key particpants about their email practices.

This period will be kept as brief as possible.

We will then take the data away for analysis.

We will replace real names with pseudonyms to protect people's identity.

At the end of the research, we will provide a summary of the results, and check whether those who have participated would like any other sort of feedback, such as a workshop.

\section{What will the data be used for?}

The data we collect from you will be used only for these purposes:

(i) research, including doctoral research; (ii) publications and presentations based on this research; and (iii) evaluation and development of workplace communication practices in your workplace.

\section{Who will have access to the data?}

All the data collected as part of this project will be stored securely at Victoria University. Only authorised members of the research team will have access to this material. Unless, you decide otherwise, the identity of your organisation will be kept confidential in any use of the material in presentations. The identities of those particpating in the research will always be kept confidential. 


\section{THE RESEARCH PROCESS - METHODS}

Aims of the LWP project

- To identify distinctive features of workplace communication in different workplaces

- To identify communication strategies of effective communicators in the organisation

- To explore the implications of the findings for workplace relationships in order to provide useful input to human resource and professional development programmes

\section{The LWP Research Team}

- Professor Janet Holmes (Project Director)

- Dr Meredith Marra (Research Officer)

- Dr Bernadette Vine (Corpus Manager)

- Miss Jackie Yeoh ( $\mathrm{PhD}$ student)

\section{Method}

The methods outlined below are provided as a basis for discussion. They are brief, but we can provide more detail if required. The final methods adopted will reflect discussion between the Company XXX and the researchers, and we will ensure that they are acceptable to all those participating in the research. Every step is subject to the agreement of those participating in the project, as well as the agreement of any individual person to be recorded.

1. Participant observation

Jackie Yeoh will undertake a short period of observation before any data collection begins.

\section{Questionnaire}

A written questionnaire will be distributed to all those interested in providing information on their workplace email activities in relation to other communicative activities.

3. Email data

- A small number of key participants will be asked to provide a sample of email data - the precise data to be agreed after discussion.

- Short interviews will be undertaken with these key participants as convenient after the email sample has been identified and provided in order to collect participants' reflections on the material provided.

- The key participants may be asked to provide reflective comments on their email activities during the process of writing and processing email.

\section{Ethics and confidentiality}

The ethical guidelines subscribed to by social scientists internationally will be observed as well as the specific guidelines of Victoria University's Human Ethics Committee.

- The participation of all contributors will be entirely voluntary.

- Material will be collected and analysed only with the agreement of contributors.

- Permission will be obtained from all authors of emails included in the data.

- The identity of those involved will be protected by the use of pseudonyms.

- Participants always have the right to decide on which material will be provided for analysis and may withdraw consent for the use of the material at any point.

- Once collected, material will be analysed only by a small team of authorised researchers. The anonymised email data will not be made available to anyone outside the research team without permission. 
- The material collected will be used as a basis for publications and presentations, and to assist in the development of training materials which could be of benefit to Company XX. Company XX will not be named in publications, except with written permission.

- All information supplied as part of this research will be kept confidential, and will not be released in the event of a request under the Official Information Act.

\section{Costs and benefits for Company XX}

- The research will contribute to a better understanding of the role of communication in organisations, and of the communicative practices engaged in by effective team members within the Company XX in particular.

- Those who agree to provide material will be committing only minimal time over and above their normal work time to the project over a period of about a month: i.e. for completing interviews and questionnaires. The data collection process will not take additional time since contributors will be providing material involving their normal workplace business. The participant observation will not entail any disruption of the normal activities of Company XX employees.

- The results of the analysis will be made available to Company XX in a form comprehensible to non-specialists.

- The research team is happy to present the results in the form of a seminar or workshop for Company XX managers/staff if this is considered desirable.

- The research team would welcome the opportunity to discuss ways in which the material gathered might be developed as input to training programmes for Company XX staff. 


\section{Additional more specific information}

1. Some specific examples of potential benefits to Company $\mathrm{XX}$

- Identification of excellent communication skills has consistently proved beneficial to people in workplaces where we have worked previously

- Identification of ways of interacting which may be distinctive to people working at Company XX - for purposes of recruitment, mentoring, modelling etc.

- Analysis of how people obtain cooperation, how a group reaches decisions, how a manager expresses a criticism etc. can raise awareness of the skills involved and provide a basis for discussion, reflection and improvement

- Analysis of the leadership styles that are preferred in your organisation with some examination of what makes them special or distinctive

- A workshop or seminar based on our findings for all your staff

\section{Confidentiality issues}

- We can be confident on the basis of more than 10 years experience that we can protect identities.

- We are not interested in content but in processes of interaction. We change references of any kind, e.g. to names, concepts, or places, which could be identifying.

- Material is locked away and real names are never used in any material.

\section{Data collection issues}

- Ideally we would like to work with up to four key participants in different roles in the organisation.

- Those who participate will be given the opportunity to talk to us afterwards in order to give us feedback on the experience.

These are ideas based on our previous work, and we would like a chance to examine them with data from an organisation that we have been told is an effective one which welcomes research.

Professor Janet Holmes 11 February 2011 


\section{Appendix 4: Consent form}

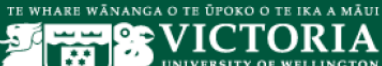

Letter of consent

Re: Consent to Participate in Research

Research Topic: Email Communication in the Workplace

Researcher: Jackie Yeoh, School of Linguistics and Applied Languages Studies, Victoria University of Wellington

I have been given and have understood an explanation of this research project. I have had an opportunity to ask questions. The answers have provided the information I need to know and have been satisfactory.

My participation is voluntary and my organisation has agreed to my participation. I understand that I may withdraw from the research up to 2 months after I provide data, without having to give reasons.

I understand that any information I provide will be kept confidential to approved researchers, the published results will not use my name, and that no opinions will be attributed to me that will identify me. I understand that all data collected will be securely stored in the archives of the Wellington Language in the Workplace Project.

I would like to receive a summary of results of this research when it is completed. [tick $(\checkmark)$ in the box if interested]

My email address is

Signed:

Date:

Name of participant

(Please print clearly) 


\section{Appendix 5: Workplace email questionnaire for participants}

\begin{tabular}{l|l} 
Workplace Email Questionnaire & Co NZ
\end{tabular}

\section{Workplace Email Questionnaire}

In this questionnaire, email refers to the sending and receiving of electronic messages. It does not refer to any other form of internet communication (for example: Facebook, Skype).

\section{A. My personal profile [tick $(\checkmark)$ the appropriate choice given]}
1. Your gender
$\square$ Male
$\square$ Female
2. Your age group
口 $21-25$
$\square 26-35$
$\square 36-44$
$\square \quad 45-54$
$\square$ above 55

3. Ethnicity
4. Is English your first language?
$\square$ Yes
No

5. What is your highest educational qualification?
$\square$ Tertiary level qualification
$\square$ Secondary level qualification
$\square \quad$ No educational qualification

6. What is your current position at work?

7. How long have you been in this position? years months

8. Relevant type of employment $\quad \square$ Full time $\quad \square$ Part-time

\section{B. Email Usage [tick $(\checkmark)$ the appropriate choice given]}

1. Do you have a work email account?
$\square$ Yes
$\square$ No

2. Do you check your email first thing in the morning when you arrive at work?
$\square$ Yes
$\square$ No
$\square$ Sometimes

3. When do you read your email messages during a regular working day?
$\square$ as soon as one arrives in my inbox
$\square$ when I am free
$\square$ at the end of the day before I leave for the day
$\square$ as and when I feel like it
$\square$ I don't read them at all 
4. When do you reply to your email messages during a regular working day?

$\square$ as soon as I have read them

$\square$ when I am free

$\square$ at the end of the day before I leave for the day

$\square$ as and when I feel like it

$\square$ I don't reply to them at all

5. For the following actions, please indicate with a tick $(\checkmark)$ in the appropriate box the frequency (listed below) of how you use your email.

$$
\begin{aligned}
& \mathbf{1}=\text { never } \\
& \mathbf{2}=\text { seldom } \\
& \mathbf{3}=\text { sometimes } \\
& \mathbf{4}=\text { often } \\
& \mathbf{5}=\text { always }
\end{aligned}
$$

a. Give instructions/ directives

b. Send information

c. Make announcements

d. Send agendas for meetings

e. Check availability for meetings

f. Make requests

g. Solve a problem

h. Resolve conflict

i. Offer feedback

j. Send compliments

k. Say thank you

I. Reply to emails

m. Seek advice

n. Other (please specify):

o. Other (please specify):

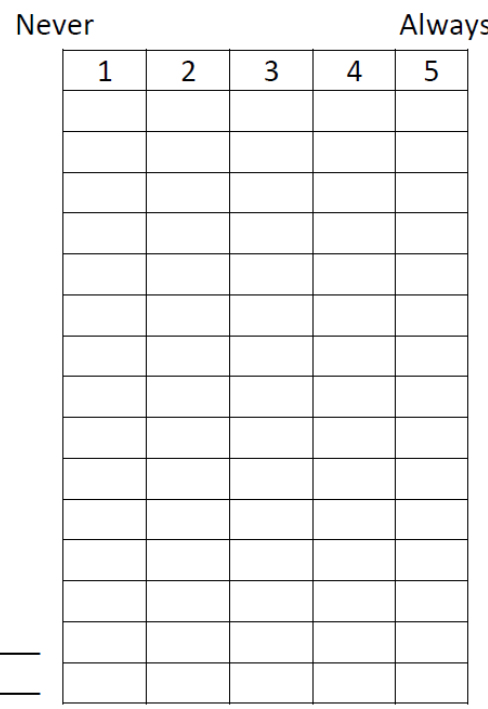

\section{Other modes of communication [tick $(\checkmark)$ the appropriate choice given]}

1. Has your use of email at work increased in the past year? $\quad \square$ Yes $\square$ No Any comment:

2. If "yes", by how much?

Big Increase (more than $50 \%$ )

Small Increase (less than 50\%) 
3. If "yes", what has been the effect on the following modes of communication over the last year? [tick $(\checkmark)$ in the appropriate column]

\begin{tabular}{|l|l|c|c|c|c|}
\hline Mode of communication & $\begin{array}{c}\text { Big } \\
\text { Decrease }\end{array}$ & $\begin{array}{c}\text { Some } \\
\text { Decrease }\end{array}$ & $\begin{array}{c}\text { No } \\
\text { change }\end{array}$ & $\begin{array}{c}\text { Some } \\
\text { Increase }\end{array}$ & $\begin{array}{c}\text { Big } \\
\text { Increase }\end{array}$ \\
\hline Telephone & & & & & \\
\hline $\begin{array}{l}\text { Face-to-face (include meeting } \\
\text { and discussion) }\end{array}$ & & & & & \\
\hline Fax & & & & & \\
\hline Letters & & & & & \\
\hline Memos & & & & & \\
\hline
\end{tabular}

4. Approximately how much time in your regular working day do you spend on the following modes of communication for work related purposes? [tick $(\checkmark)$ in the appropriate column]

\begin{tabular}{|l|l|l|l|l|l|}
\hline & $\begin{array}{c}\text { None } \\
\text { at all }\end{array}$ & $\begin{array}{c}\mathbf{0 - 3 0} \\
\text { minutes }\end{array}$ & $\begin{array}{c}\mathbf{3 0} \text { minutes } \\
\text { to } \mathbf{1} \text { hour }\end{array}$ & $\begin{array}{c}\mathbf{1 - 2} \\
\text { hours }\end{array}$ & $\begin{array}{c}\text { More than } \\
\mathbf{2} \text { hours }\end{array}$ \\
\hline Talk to someone in person & & & & & \\
\hline Attend meetings & & & & & \\
\hline Talk on telephone & & & & & \\
\hline Read, write and respond to email & & & & & \\
\hline $\begin{array}{l}\text { Write letters, memos, faxes or } \\
\text { written notes }\end{array}$ & & & & & \\
\hline
\end{tabular}

5. How effective for you in your work is each mode of communication for each of the following situations? For each mode of communication, please circle the answer that best expresses your position.

$$
\begin{aligned}
& \mathbf{N E}=\text { not effective at all } \\
& \mathbf{Q E}=\text { quite effective } \\
& \mathbf{E}=\text { effective } \\
& \mathbf{V E}=\text { very effective } \\
& \mathbf{E E}=\text { extremely effective }
\end{aligned}
$$

a) Situation: Delivering announcements

\begin{tabular}{|l|ccccc|}
\hline Face-to-face & NE & QE & E & VE & EE \\
\hline Telephone conversations & NE & QE & E & VE & EE \\
\hline Email & NE & QE & E & VE & EE \\
\hline Other written forms & NE & QE & E & VE & EE \\
\hline
\end{tabular}

b) Situation: Delivering good news

\begin{tabular}{|l|ccccc|}
\hline Face-to-face & NE & QE & E & VE & EE \\
\hline Telephone conversations & NE & QE & E & VE & EE \\
\hline Email & NE & QE & E & VE & EE \\
\hline Other written forms & NE & QE & E & VE & EE \\
\hline
\end{tabular}


c) Situation: Delivering bad news

\begin{tabular}{|l|ccccc|}
\hline Face-to-face & NE & QE & E & VE & EE \\
\hline Telephone conversations & NE & QE & E & VE & EE \\
\hline Email & NE & QE & E & VE & EE \\
\hline Other written forms & NE & QE & E & VE & EE \\
\hline
\end{tabular}

d) Situation: Delivering information

\begin{tabular}{|l|ccccc|}
\hline Face-to-face & NE & QE & E & VE & EE \\
\hline Telephone conversations & NE & QE & E & VE & EE \\
\hline Email & NE & QE & E & VE & EE \\
\hline Other written forms & NE & QE & E & VE & EE \\
\hline
\end{tabular}

e) Situation: Giving instructions/directives

\begin{tabular}{|l|ccccc|}
\hline Face-to-face & NE & QE & E & VE & EE \\
\hline Telephone conversations & NE & QE & E & VE & EE \\
\hline Email & NE & QE & E & VE & EE \\
\hline Other written forms & NE & QE & E & VE & EE \\
\hline
\end{tabular}

f) Situation: Confirming information

\begin{tabular}{|l|ccccc|}
\hline Face-to-face & NE & QE & E & VE & EE \\
\hline Telephone conversations & NE & QE & E & VE & EE \\
\hline Email & NE & QE & E & VE & EE \\
\hline Other written forms & NE & QE & E & VE & EE \\
\hline
\end{tabular}

g) Situation: Giving advice

\begin{tabular}{|l|ccccc|}
\hline Face-to-face & $\mathrm{NE}$ & $\mathrm{QE}$ & $\mathrm{E}$ & $\mathrm{VE}$ & $\mathrm{EE}$ \\
\hline Telephone conversations & $\mathrm{NE}$ & $\mathrm{QE}$ & $\mathrm{E}$ & $\mathrm{VE}$ & $\mathrm{EE}$ \\
\hline Email & $\mathrm{NE}$ & $\mathrm{QE}$ & $\mathrm{E}$ & $\mathrm{VE}$ & $\mathrm{EE}$ \\
\hline Other written forms & $\mathrm{NE}$ & $\mathrm{QE}$ & $\mathrm{E}$ & $\mathrm{VE}$ & $\mathrm{EE}$ \\
\hline
\end{tabular}

h) Situation: Resolving conflict

\begin{tabular}{|l|ccccc|}
\hline Face-to-face & NE & QE & E & VE & EE \\
\hline Telephone conversations & NE & QE & E & VE & EE \\
\hline Email & NE & QE & E & VE & EE \\
\hline Other written forms & NE & QE & E & VE & EE \\
\hline
\end{tabular}

i) Situation: Seeking clarification

\begin{tabular}{|l|ccccc|}
\hline Face-to-face & NE & QE & E & VE & EE \\
\hline Telephone conversations & NE & QE & E & VE & EE \\
\hline Email & NE & QE & E & VE & EE \\
\hline Other written forms & NE & QE & E & VE & EE \\
\hline
\end{tabular}




\section{Your attitude towards and patterns of use of email}

In this section, you are given a number of statements. For each statement, please circle the answer that best expresses your position on each issue.

$$
\begin{aligned}
& \text { SD }=\text { Strongly Disagree } \\
& \mathbf{D}=\text { Disagree } \\
& \mathrm{N}=\text { Neither Agree nor Disagree } \\
& \mathbf{A}=\text { Agree } \\
& \text { SA }=\text { Strongly Agree }
\end{aligned}
$$

\begin{tabular}{|c|c|c|c|c|c|c|}
\hline 1 & Email is a waste of time. & SD & $\mathrm{D}$ & $\mathrm{N}$ & A & SA \\
\hline 2 & $\begin{array}{l}\text { I like to use email to correspond with my } \\
\text { colleagues and manager/ supervisor. }\end{array}$ & SD & $\mathrm{D}$ & $\mathrm{N}$ & A & SA \\
\hline 3 & I feel competent using email. & SD & $\mathrm{D}$ & $\mathrm{N}$ & A & SA \\
\hline 4 & $\begin{array}{l}\text { It is important to start email messages with a } \\
\text { greeting or the person's name. }\end{array}$ & SD & $\mathrm{D}$ & $\mathrm{N}$ & A & SA \\
\hline 5 & I use email when I want to avoid confrontation. & SD & $\mathrm{D}$ & $\mathrm{N}$ & A & SA \\
\hline 6 & $\begin{array}{l}\text { I feel more comfortable using email to } \\
\text { communicate with people I know than with } \\
\text { people I don't know. }\end{array}$ & SD & $\mathrm{D}$ & $\mathrm{N}$ & A & SA \\
\hline 7 & $\begin{array}{l}\text { I prefer to talk to people face-to-face or via the } \\
\text { telephone. }\end{array}$ & SD & $\mathrm{D}$ & $\mathrm{N}$ & A & SA \\
\hline 8 & I find it difficult to write email messages. & SD & $\mathrm{D}$ & $\mathrm{N}$ & A & SA \\
\hline 9 & $\begin{array}{l}\text { I send email to colleagues who are at close } \\
\text { proximity. }\end{array}$ & SD & $\mathrm{D}$ & $\mathrm{N}$ & A & SA \\
\hline 10 & $\begin{array}{l}\text { I think email messages can be informally } \\
\text { written. }\end{array}$ & SD & $\mathrm{D}$ & $\mathrm{N}$ & A & SA \\
\hline 11 & $\begin{array}{l}\text { It is important to reply promptly to email } \\
\text { messages. }\end{array}$ & SD & $D$ & $\mathrm{~N}$ & A & SA \\
\hline 12 & $\begin{array}{l}\text { I get annoyed with people who use capital } \\
\text { letters, bold, underline and red fonts in their } \\
\text { emails messages. }\end{array}$ & SD & $\mathrm{D}$ & $\mathrm{N}$ & A & SA \\
\hline 13 & $\begin{array}{l}\text { It is important to end the email message with a } \\
\text { sign off. }\end{array}$ & SD & $\mathrm{D}$ & $\mathrm{N}$ & A & SA \\
\hline 14 & $\begin{array}{l}\text { The mode of communication I choose depends } \\
\text { on the person who I am going to communicate. }\end{array}$ & SD & $\mathrm{D}$ & $\mathrm{N}$ & $A$ & SA \\
\hline 15 & I spend a lot of time drafting an email message. & SD & $\mathrm{D}$ & $\mathrm{N}$ & A & SA \\
\hline 16 & Email gets work done faster. & SD & $\mathrm{D}$ & $\mathrm{N}$ & A & SA \\
\hline 17 & $\begin{array}{l}\text { I don't use greeting when sending email } \\
\text { messages to close colleagues. }\end{array}$ & SD & $\mathrm{D}$ & $\mathrm{N}$ & $A$ & SA \\
\hline
\end{tabular}


Workplace Email Questionnaire $\quad$ Co NZ

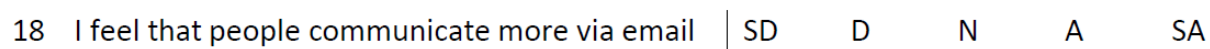
and talk less to each other.

\begin{tabular}{|c|c|c|c|c|c|c|}
\hline 19 & Email saves a lot of time. & SD & $\mathrm{D}$ & $\mathrm{N}$ & A & SA \\
\hline 20 & $\begin{array}{l}\text { When I reply to an email sent to a group, I } \\
\text { always click 'reply all'. }\end{array}$ & SD & $\mathrm{D}$ & $\mathrm{N}$ & A & SA \\
\hline 21 & $\begin{array}{l}\text { I prefer to write email than to talk to people } \\
\text { face-to-face. }\end{array}$ & SD & $\mathrm{D}$ & $\mathrm{N}$ & A & SA \\
\hline 22 & Email is annoying. & SD & $\mathrm{D}$ & $\mathrm{N}$ & A & SA \\
\hline 23 & I always send an email with a subject heading. & SD & $\mathrm{D}$ & $\mathrm{N}$ & A & SA \\
\hline 24 & $\begin{array}{l}\text { I have the reader in mind while crafting my } \\
\text { email message. }\end{array}$ & SD & $\mathrm{D}$ & $\mathrm{N}$ & A & SA \\
\hline 25 & $\begin{array}{l}\text { I prefer to use email rather than other means } \\
\text { of communication to make requests to my } \\
\text { boss. }\end{array}$ & SD & $\mathrm{D}$ & $\mathrm{N}$ & A & SA \\
\hline 26 & $\begin{array}{l}\text { I don't sign off email messages directed to close } \\
\text { colleagues. }\end{array}$ & SD & $\mathrm{D}$ & $\mathrm{N}$ & A & SA \\
\hline 27 & Email creates misunderstandings. & SD & $\mathrm{D}$ & $\mathrm{N}$ & A & SA \\
\hline 28 & I never delete emails without opening them. & SD & $\mathrm{D}$ & $\mathrm{N}$ & A & SA \\
\hline 29 & $\begin{array}{l}\text { I always change or delete other people's } \\
\text { messages before forwarding them on. }\end{array}$ & SD & $\mathrm{D}$ & $\mathrm{N}$ & A & SA \\
\hline 30 & $\begin{array}{l}\text { Email is the best way to communicate with my } \\
\text { manager/supervisor. }\end{array}$ & SD & $\mathrm{D}$ & $\mathrm{N}$ & A & SA \\
\hline 31 & $\begin{array}{l}\text { I write longer emails to colleagues who are not } \\
\text { close to me. }\end{array}$ & SD & $\mathrm{D}$ & $\mathrm{N}$ & A & SA \\
\hline 32 & $\begin{array}{l}\text { I always proof read my email message before } \\
\text { sending it. }\end{array}$ & SD & $\mathrm{D}$ & $\mathrm{N}$ & A & SA \\
\hline 33 & $\begin{array}{l}\text { I copy (cc) my email to my manager/supervisor } \\
\text { when it is an important email. }\end{array}$ & SD & $\mathrm{D}$ & $\mathrm{N}$ & A & SA \\
\hline 34 & $\begin{array}{l}\text { I always send emails which are as short as } \\
\text { possible. }\end{array}$ & SD & $\mathrm{D}$ & $\mathrm{N}$ & A & SA \\
\hline 35 & I never send email with only a subject heading. & SD & $\mathrm{D}$ & $\mathrm{N}$ & A & SA \\
\hline 36 & $\begin{array}{l}\text { I always use capital letter, bold or underline } \\
\text { when I want to highlight important information } \\
\text { in my email message. }\end{array}$ & SD & $\mathrm{D}$ & $\mathrm{N}$ & A & SA \\
\hline 37 & $\begin{array}{l}\text { I prefer to use email rather than other means } \\
\text { of communication to make requests of my } \\
\text { colleagues. }\end{array}$ & SD & $\mathrm{D}$ & $\mathrm{N}$ & A & SA \\
\hline 38 & $\begin{array}{l}\text { I get annoyed with people who never reply my } \\
\text { email on the same day I wrote to them. }\end{array}$ & SD & $\mathrm{D}$ & $\mathrm{N}$ & A & SA \\
\hline
\end{tabular}


Workplace Email Questionnaire $\quad$ Co NZ

\begin{tabular}{ll|lllll}
\hline 39 & $\begin{array}{l}\text { I only write one sentence email messages to } \\
\text { close colleagues. }\end{array}$ & SD & D & N & A & SA \\
\hline $40 \begin{array}{l}\text { When I reply to emails sent to a group, I click } \\
\text { only 'reply' rather than 'reply all'. }\end{array}$ & SD & D & N & A & SA \\
\hline
\end{tabular}

THANK YOU FOR YOUR TIME

Are you willing to be interviewed about your experience of email? If yes, please write your name and contact number in the space provided below.

Name:

Tel. Extn:

Please add here any additional comments you would like to make about your use of email at work. If the comment relates to a specific question in the questionnaire, please indicate the question number. Please continue on the back of this page if necessary. Thank you. 


\section{Appendix 6 - Chapter 6 Emails}

Email 6.19 (SA31) NZ1

From: Andy

Sent: Friday, 4 February 2011 6:06 p.m.

To: Jacinta; Jean

Cc: Joline; Sally

Subject: RE: Car parks

All - we are a small office and we should be able to have conversations with each other face to face, without having to send out formal emails to everyone. Especially when it concerns what I consider to be a relatively small issue which does not affect the running of the business. I am doing this via email so you all get the same message.

If you have an issue with someone - just go see them. If you feel like you are still having issues after the conversation, then come see me.

I trust the lessons we learned as part of the team building about communicating with our colleagues can be used moving forward.

Email 6.20 (SM16) NZ2

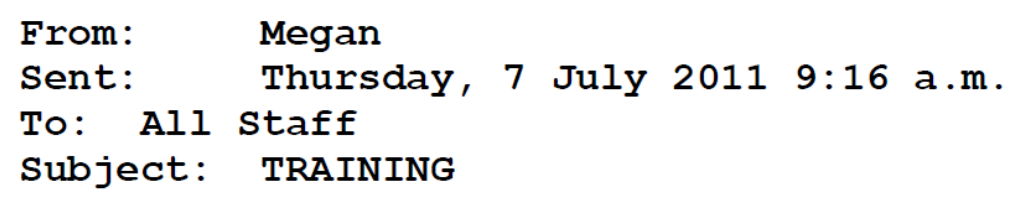

This is not good enough. A lot of time and effort is put into organising the training and it also costs a lot of money.

I sent out a reminder on Friday thinking that this would be enough to remind everyone. Some people were sent to the wrong course on the wrong day and others just did not turn up. Surely we are all capable of reading an email and organising our staff to attend the correct course on the correct day.

As you can probably tell by the tone of this email I am pretty annoyed -

If for any reason anyone is on a course that does not suit

surely it is not too much to ask that I am notified in time to make the necessary changes.

Megan 
Email $6.21(\mathrm{SCO5}) \mathrm{M}$

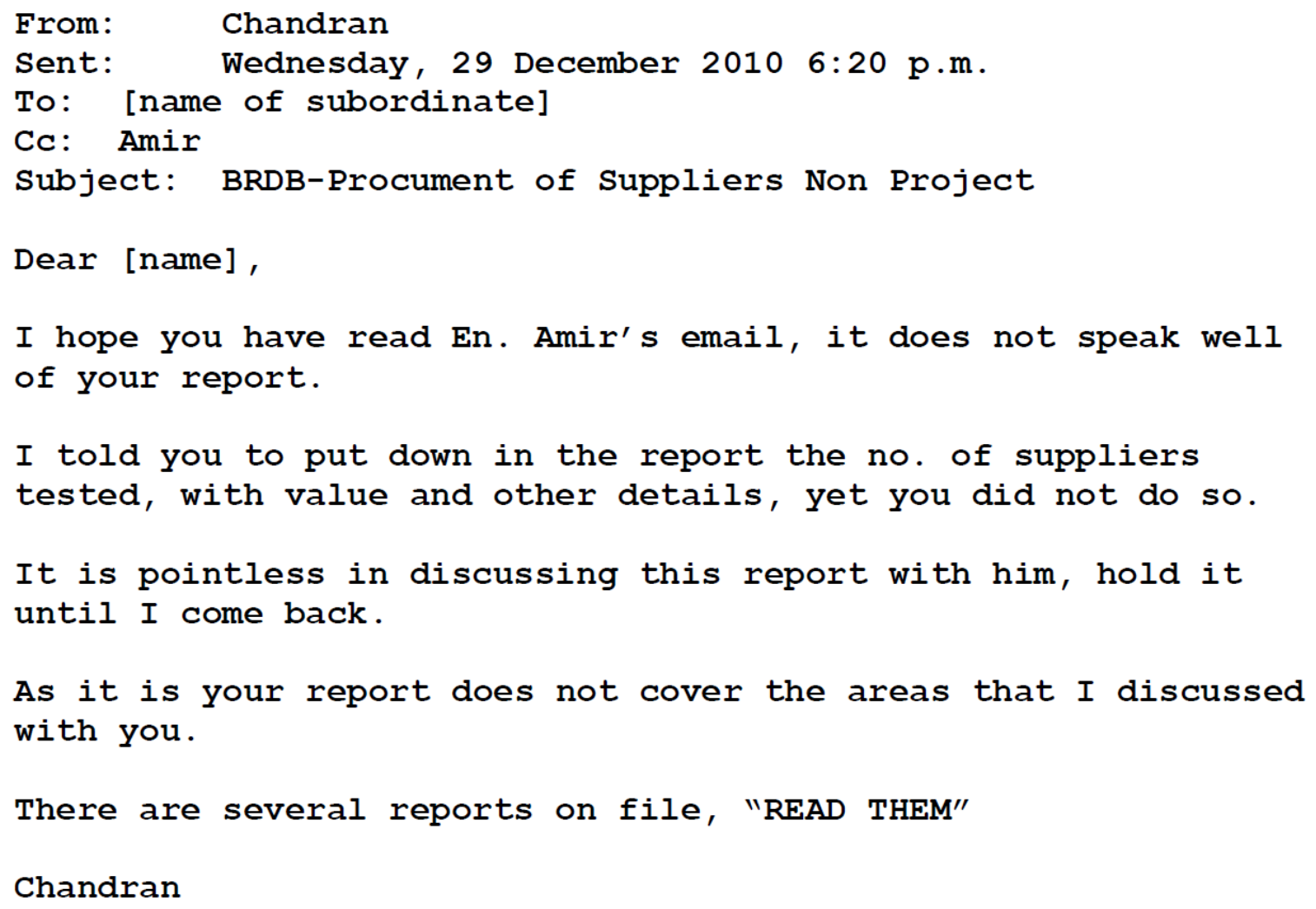

